\title{
Development of a Safety-Oriented Interaction Strategy and Compliant Limb Sensor System for a Gait Rehabilitation Robot
}

by

Colin Miyata, B.Eng.

A thesis submitted to the Faculty of Graduate and Postdoctoral Affairs

in partial fulfillment of the requirements for the degree of

Master of Applied Science

in

Biomedical Engineering

Carleton University

Ottawa, Ontario

July 28th, 2015

(C) 2015

Colin Miyata 


\section{Abstract}

Robots are expanding into new fields through human-robot interaction, however this has created safety concerns due to collisions. This research develops the Safety-Oriented Interaction Strategy (SOIS) that detects collisions by measuring deformation of a compliant limb sensor (CLS) and yields using a zero force controller that directly feeds back CLS deformation. Using a 1DoF LTI model, controller stability was proven and the reduction of impedance and CLS deformation was analyzed. The effect of nonlinearities on the controller was simulated. Stiction was found to generate oscillations that could be eliminated by thresholding CLS feedback. The proposed controller displayed similar deformation and force reduction as admittance control during experiments while remaining stable. Potentiometer and optical based planar CLSs were prototyped and the potentiometer based CLS was more accurate (average errors $<1 \mathrm{~mm} / 0.5^{\circ}$ ). However, the potentially lightweight optical based sensor can follow general motion, and with refinement can be used in future designs. 
To my wife, Joie. It has been a hectic, stressful and exciting time and I am always thankful for your love and support. 


\section{Acknowledgments}

I would like to thank my supervisor, Mojtaba Ahmadi, for his guidance and encouragement throughout my thesis. I am also thankful for all the help I have received from Kyle Chisholm and Jennifer Baba in the ABL lab throughout my research. Finally a special thanks to my family and friends who have supported me throughout my studies. 


\section{Table of Contents}

Abstract

Acknowledgments $\quad$ iv

Table of Contents $\quad$ v

List of Tables ix

List of Figures $\quad$ xi

Nomenclature $\quad$ xix

1 Introduction 1

1.1 Motivation ..................... 2

1.2 Background ...................... . . . 4

1.2.1 Literature Review . . . . . . . . . . . . . . . . . 4

1.2.2 Project History . . . . . . . . . . . . . . . . . . . . . . . . 19

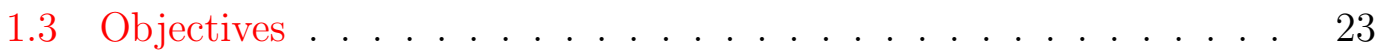

1.4 Outline of Thesis . . . . . . . . . . . . . . . . . 24

2 The Safety Oriented Interaction Strategy Control Law for the 
$\begin{array}{ll}\text { Virtual Gait Rehabilitation Robot } & 27\end{array}$

2.1 Control Objectives . . . . . . . . . . . . . . . . 28

2.2 Control Methodology . . . . . . . . . . . . . . . . 33

2.2.1 Deficits with admittance and impedance control using the

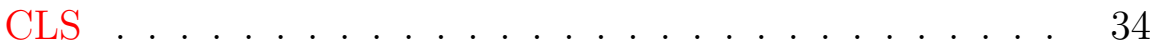

2.2.2 Proposed SOIS Control Law . . . . . . . . . . . . . . . . 38

2.3 Linear Time Invariant Controller Analysis . . . . . . . . . . . . . 42

2.3.1 System Model . . . . . . . . . . . . . . . . 43

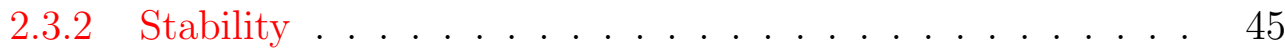

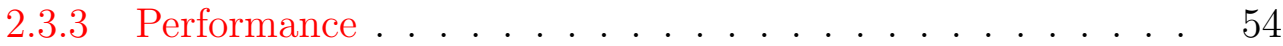

2.4 Summary . . . . . . . . . . . . . . . . 62

3 Investigation of the Single Degree of Freedom Control Law $\begin{array}{ll}\text { through Simulation and Experimentation } & 64\end{array}$

3.1 Design Procedure . . . . . . . . . . . . . . . 65

3.2 Hardware . . . . . . . . . . . . . . . . . . 76

3.2.1 1DoF test platform . . . . . . . . . . . . 77

3.2.2 1DoF Compliant Limb Sensor . . . . . . . . . . . . . 79

3.3 Control Design ....................... 82

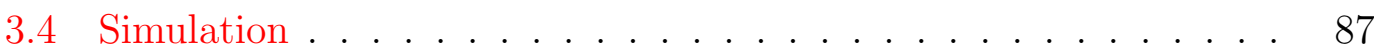

3.4.1 System Model . . . . . . . . . . . . . . . 89

3.4 .2 1DoF LTI system . . . . . . . . . . . . . . . . . 91

3.4.3 1DoF linear system with force saturation . . . . . . . . 92

3.4.4 1DoF linear system with varying sampling frequencies . . . 96

3.4.5 1DoF linear system with sensor noise . . . . . . . . . . . 96 
3.4.6 1DoF linear system with stiction $\ldots \ldots \ldots \ldots$

3.5 Experimental Validation . . . . . . . . . . . . . . . . . 101

3.5.1 Impact Testing . . . . . . . . . . . . . . . . . . . 103

3.5.2 Interaction Testing . . . . . . . . . . . . . . . 104

3.6 Summary . . . . . . . . . . . . . . . . . 106

4 Initial Development of the Three Degree of Freedom Compliant

$\begin{array}{ll}\text { Limb Sensor } & 109\end{array}$

4.1 Potentiometer Based Compliant Limb Sensor . . . . . . . . . . 110

4.1 .1 Mechanical Design . . . . . . . . . . . . . . . . . . 111

4.1 .2 Hardware . . . . . . . . . . . . . . . . . . . 116

4.1 .3 Sensor Calibration . . . . . . . . . . . . . . . 118

4.1 .4 Performance . . . . . . . . . . . . . . . . . . . . . 121

4.1 .5 Discussion . . . . . . . . . . . . . . . . . . 123

4.2 Optical Sensor Based Compliant Limb Sensor _ . . . . . . . . 126

4.2 .1 Sensor Design . . . . . . . . . . . . . . . . . . . 127

4.2 .2 LTV sensor modelling . . . . . . . . . . . . . . . 128

4.2 .3 CLS calibration . . . . . . . . . . . . . . . . . . 131

4.2.4 Pose Estimation and Performance . . . . . . . . . . . . 132

4.2 .5 Discussion . . . . . . . . . . . . . . . . . . . 133

4.3 Summary . . . . . . . . . . . . . . . . 136

5 Conclusions and Future Work 138

5.1 Conclusions . . . . . . . . . . . . . . . . . . . . . . . . 139

5.2 Future Work . . . . . . . . . . . . . . . . . . . . . . . 141 
A.1 $X_{R}(s)$ for the isolated system $\left(F_{\text {ext }}=0\right)$ with a PID position controller and nonzero initial conditions . . . . . . . . . . . . 155

A.2 Calibration of damping coefficient for the 1DoF test platform . . . 156

A.3 Calibration of damping coefficient for the 1DoF CLS . . . . . . . 158

A.4 Parameters for Head Impact Model . . . . . . . . . . . . . . . . . 160

A.5 Additional Simulation Results: . . . . . . . . . . . . . . 165 


\section{List of Tables}

1.1 Peak joint parameters of the ViGRR prototype. . . . . . . . . . .

3.1 The effect of varying $\Delta x_{R \max }, P O_{\max }$ and $V_{d \max }$ on the required controller conditions. - indicates no effect, $+\propto$ indicates the condition is relaxed by increasing the value and $-\propto$ indicates the condition is relaxed by decreasing the value. . . . . . . . . .

3.2 Design specifications for the $1 \mathrm{DoF}$ test platform and CLS to be used in controller design . . . . . . . . . . . . . . . .

3.3 Parameters for various simulated environments. . . . . . . . . .

3.4 Summarizing statistics of the maximum impact forces over 4 trials for the proposed and admittance controllers during impact at varying angular velocities. . . . . . . . . . . . . .

3.5 Summarizing statistics of the maximum CLS deformations over 4 trials for the proposed and admittance controllers during impact at varying angular velocities. . . . . . . . . . . . . .

3.6 Stiffness constants of the tension and compression springs employed to connect the CLS to the fixed post. . . . . . . . . . . . 105

4.1 Components of joint axes in the general coordinate frame for the rest position. . . . . . . . . . . . . . . . . 120 
4.2 Factor to convert from changes in voltage ratio to joint displacements for each link . . . . . . . . . . . . . . . . . . . 121

4.3 Summarizing statistics on the accuracy of the potentiometer based CLS to track motion and orientation of the external shell. . . . . 123

4.4 Summarizing statistics of errors in tracking motion and orientation

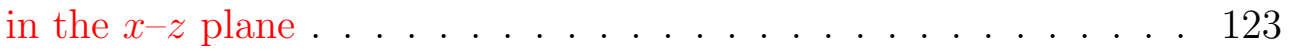

4.5 Statistics summarizing the accuracy of the optical CLS to predict motion in the plane of interest for given ranges of deformation $D .133$

A.1 Selected parameters for the head impact model . . . . . . . . . 161 


\section{List of Figures}

1.1 Description of possible injuries due to physical contact between a human and robot $[1] \ldots \ldots \ldots 6$

1.2 Decision tree to determine which collision reaction should be applied in a given situation $[2] \ldots \ldots \ldots$

1.3 Representative block diagram of impedance control. . . . . . . . . 14

1.4 Representative block diagram of admittance control. . . . . . . . . 15

1.5 The ViGRR performing a rehabilitation exercise [3] . . . . . . . . 20

1.6 Schematic presenting the CLS concept. . . . . . . . . . . . . . . . 22

2.1 Simplified block diagrams for the generation of $\vec{x}_{d e s}$ in (a) admittance control and (b) the proposed direct feedback. . . . . . . . . 39

2.2 The block diagram of the SOIS controller for the ViGRR. . . . . . 40

2.3 LTI model for the combined 1 DoF robot and CLS system . . . . 45

2.4 Effect of system stiffness and damping on $V_{\max } \ldots \ldots . . . . . .57$

2.5 Impedance of $1 \mathrm{DoF}$ LTI system with $K_{d}=80 \mathrm{~N} \mathrm{~s} \mathrm{~m}^{-1}$ for varying $K_{p}$ (blue $=500 \mathrm{~N} \mathrm{~m}^{-1}$, red $=1000 \mathrm{~N} \mathrm{~m}^{-1}$, black $=2000 \mathrm{~N} \mathrm{~m}^{-1}$ and

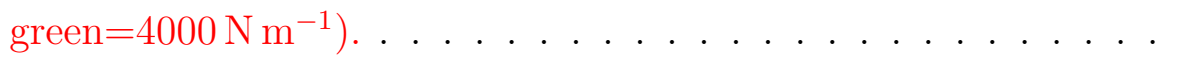


2.6 Impedance of $\left(M_{R} s^{2}+\left(B_{R}+B_{S}+K_{d}\right) s+K_{S}+K_{p}\right)^{-1}$ with $K_{d}=$ $80 \mathrm{~N} \mathrm{sm}^{-1}$ for varying $K_{p} \quad\left(\right.$ blue $=500 \mathrm{~N} \mathrm{~m}^{-1}, \quad$ red $=1000 \mathrm{~N} \mathrm{~m}^{-1}$, black $=2000 \mathrm{~N} \mathrm{~m}^{-1}$ and green $=4000 \mathrm{~N} \mathrm{~m}^{-1}$. . . . . . . . . . . . . 60

2.7 Impedance of 1DoF LTI system with $K_{p}=1000 \mathrm{~N} \mathrm{~m}^{-1}$ for varying $K_{d}$ (blue $=20 \mathrm{~N} \mathrm{~s} \mathrm{~m}^{-1}, \operatorname{red}=40 \mathrm{~N} \mathrm{~s} \mathrm{~m}^{-1}$, black $=80 \mathrm{~N} \mathrm{~s} \mathrm{~m}^{-1}$ and green $\left.=160 \mathrm{~N} \mathrm{~m}^{-1}\right) . \ldots \ldots \ldots \ldots \ldots$

2.8 Impedance of $\left(M_{R} s^{2}+\left(B_{R}+B_{S}+K_{d}\right) s+K_{S}+K_{p}\right)^{-1}$ with $K_{p}=$ $1000 \mathrm{~N} \mathrm{~m}^{-1}$ for varying $K_{d} \quad\left(\right.$ blue $=20 \mathrm{~N} \mathrm{~s} \mathrm{~m}^{-1}$, red $=40 \mathrm{~N} \mathrm{~s} \mathrm{~m}^{-1}$, black $=80 \mathrm{~N} \mathrm{~s} \mathrm{~m}^{-1}$ and green $\left.=160 \mathrm{~N} \mathrm{~m}^{-1}\right) \ldots \ldots$. . . . . . . . 61

3.1 Summarizing flow chart of the four step design procedure. . . . . 68

3.2 Labelled photograph of the 1DoF test platform . . . . . . . . . . 78

3.3 1DoF compliant limb sensor . . . . . . . . . . . . . . . . . 80

3.4 Force-displacement characteristics for 1DoF CLS . . . . . . . . . 81

3.5 The range of allowable $K_{d}$ and $K_{p}$ values for each restriction with blue indicating the allowable values. . . . . . . . . . . . . . 85

3.6 Range of allowable $K_{p}$ and $K_{d}$ gains incorporating all restrictions with blue indicating allowable gains. . . . . . . . . . . . . . 86

3.7 Results of the exhaustive search to minimize the cost function. . . 87

3.8 Bode diagram of the controlled (blue) and original (red) impedance of the 1 DoF robot. . . . . . . . . . . . . . 88

3.9 Linear model employed for current simulations. . . . . . . . . . . 89

3.10 CLS deformation results of the uncontrolled (red) and controlled (blue) 1DoF linear system interacting with varying environments.

3.11 Robot motion results of the uncontrolled (red) and controlled (blue) 1DoF linear system interacting with varying environments. 
3.12 CLS deformation results of the uncontrolled (red) and controlled (blue) 1DoF linear system impacting a rigid environment. . . . .

3.13 Contact forces for 1DoF linear system, both controlled (blue) and uncontrolled (red) when impacting the employed head model. . . 95

3.14 Sample controller behaviour subject to force saturation at $166.34 \mathrm{~N}$ (blue), $100 \mathrm{~N}$ (red) and $50 \mathrm{~N}$ (green). . . . . . . . . . 95

3.15 Sample controller behaviour subject to discrete control at $2 \mathrm{kHz}$ (blue), $1 \mathrm{kHz}$ (red) and $200 \mathrm{~Hz}$ (green). . . . . . . . . . .

3.16 Sample controller behaviour subject to discrete control at $2 \mathrm{kHz}$ with the sampled measurement noise (blue), $10 \times$ the measurement noise (red) and $100 \times$ the measurement noise (green) . . . . . . . 98

3.17 Sample controller behaviour with the introduction $4 \mathrm{~N}$ (blue), $6 \mathrm{~N}$ (red) and $8 \mathrm{~N}$ (green) of static friction. . . . . . . . . . . 100

3.18 System response in interacting with environment 5 with a threshold of $0.0015 \mathrm{~m}$. The CLS stiction was set 4 N . . . . . . . . . 101

3.19 Sample force and deformation measurements for impacts at $40^{\circ} \mathrm{s}^{-1}$ using the admittance (blue) and proposed (red) control laws. . . . 104

3.20 Measured deformation of the CLS during interaction between the environment and the proposed control law. . . . . . . . . . . . 106

3.21 Measured deformation of the CLS during interaction between the environment and the admittance control law. . . . . . . . . . . 107

3.22 Sample unstable behaviour of the admittance controller for reduced damping in the admittance model. . . . . . . . . . . . . . . . 108

4.1 Series of joints in the potentiometer based CLS. . . . . . . . . . . 111 
4.2 The implementation of the open loop kinematic chain for the potentiometer based CLS. . . . . . . . . . . . . . . . . 112

4.3 Prototype of the potentiometer based CLS: a) front view and b) side view. . . . . . . . . . . . . . . . . . . 117

4.4 Circuit used for potentiometer measurement. VOUT is measured using a PCI 6229 data acquisition card and V+ is set to $9.7 \mathrm{~V}$. . . 117

4.5 Paths of the measured (blue) and estimated (red) motion of the point in 3D space. . . . . . . . . . . . . . . .

4.6 Measured (blue) and estimated (red) motion and orientations in the $x-z$ plane. . . . . . . . . . . . . . . . . . . . . . . . . . . . . . 124

4.7 Overview of the optical sensor based CLS: a) Schematic of prototype, b) labelled photograph of constructed prototype. . . . . . . 128

4.8 The parameters of the relative position between the LED and LTV sensor affecting the LTV output voltage. . . . . . . . . . . . .

4.9 Sample curve fits for the effect of a) $r$, b) $\theta$ and c) $\phi$ on the LTV sensor output. . . . . . . . . . . . . . . . 131

4.10 Measured (blue) and estimated (red) motion and orientations in the $\mathrm{x}-\mathrm{z}$ plane. . . . . . . . . . . . . . . . . . . . . 134

A.1 Labelled photograph of commanded torque versus output velocity to determine system damping . . . . . . . . . . . . 157

A.2 Curve fit of system response to an initial velocity of $30^{\circ}$ (a) without the arm, (b) with the arm, and (c) with the arm and an added mass.158

A.3 Sample CLS model fit curves for added masses (a) $M=1.027 \mathrm{~kg}$ and (b) $M=1.985 \mathrm{~kg} \ldots \ldots \ldots$. . . . . . . . . . 160 
A.4 CLS deflection results of the controlled system with varying imposed saturation forces interacting with the environment. The blue, red and green lines indicate $166.34 \mathrm{~N}, 100 \mathrm{~N}$ and $50 \mathrm{~N}$ saturation forces, respectively. . . . . . . . . . . . . . . . . . . . 162

A.5 Robot motion results of the controlled system with varying saturation forces interacting with the environment. The blue, red and green lines indicate $166.34 \mathrm{~N}, 100 \mathrm{~N}$ and $50 \mathrm{~N}$ saturation forces, respectively. . . . . . . . . . . . . . . . . 163

A.6 CLS deflection results of the controlled system with varying saturation forces impacting a rigid environment. The blue, red and green lines indicate $166.34 \mathrm{~N}, 100 \mathrm{~N}$ and $50 \mathrm{~N}$ saturation forces, respectively.164

A.7 Robot motion results of the controlled system with varying saturation forces impacting a rigid environment. The blue, red and green lines indicate $166.34 \mathrm{~N}, 100 \mathrm{~N}$ and $50 \mathrm{~N}$ saturation forces, respectively.164

A.8 Contact force results of the controlled system with varying saturation forces impacting the head model. The blue, red and green lines indicate $166.34 \mathrm{~N}, 100 \mathrm{~N}$ and $50 \mathrm{~N}$ saturation forces, respectively. 165

A.9 CLS deflection results of the discretely controlled system with varying sampling frequencies interacting with the environment. The blue, red and green lines indicate $2 \mathrm{kHz}, 1 \mathrm{kHz}$ and $200 \mathrm{~Hz}$ sampling frequencies, respectively. . . . . . . . . . . . . 166

A.10 Robot motion results of the discretely controlled system with varying sampling frequencies interacting with the environment. The blue, red and green lines indicate $2 \mathrm{kHz}, 1 \mathrm{kHz}$ and $200 \mathrm{~Hz}$ sampling frequencies, respectively. . . . . . . . . . . . . . 167 
A.11 CLS deflection results of the discretely controlled system with varying sampling frequencies impacting a rigid environment. The blue, red and green lines indicate $2 \mathrm{kHz}, 1 \mathrm{kHz}$ and $200 \mathrm{~Hz}$ sampling frequencies, respectively. . . . . . . . . . . . . . . . . . . . . 168

A.12 Robot motion results of the discretely controlled system with varying sampling frequencies impacting a rigid environment. The blue, red and green lines indicate $2 \mathrm{kHz}, 1 \mathrm{kHz}$ and $200 \mathrm{~Hz}$ sampling frequencies, respectively. . . . . . . . . . . . . . . 168

A.13 Contact force results of the discretely controlled system with varying sampling frequencies impacting the head model. The blue, red and green lines indicate $2 \mathrm{kHz}, 1 \mathrm{kHz}$ and $200 \mathrm{~Hz}$ sampling frequencies, respectively. . . . . . . . . . . . . . . 169

A.14 CLS motion results of the discretely controlled system with varying levels of sensor noise interacting with the environment. The blue, red and green lines indicate the measured noise, $10 \times$ the measured noise and $100 \times$ the measured noise, respectively. . . . . . . . . . 170

A.15 Robot motion results of the discretely controlled system with varying levels of sensor noise interacting with the environment. The blue, red and green lines indicate the measured noise, $10 \times$ the measured noise and $100 \times$ the measured noise, respectively. . . . . 171

A.16 CLS deflection results of the discretely controlled system with levels of sensor noise impacting a rigid environment. The blue, red and green lines indicate the measured noise, $10 \times$ the measured noise and $100 \times$ the measured noise, respectively. . . . . . . . . . 172 
A.17 Robot motion results of the discretely controlled system with levels of sensor noise impacting a rigid environment. The blue, red and green lines indicate the measured noise, $10 \times$ the measured noise and $100 \times$ the measured noise, respectively. . . . . . . . . . . 172

A.18 Contact force results of the discretely controlled system with levels of sensor noise impacting the head model. The blue, red and green lines indicate the measured noise, $10 \times$ the measured noise and $100 \times$ the measured noise, respectively. . . . . . . . . . . .

A.19 CLS motion results of the controlled system with varying magnitudes of dry friction interacting with the environment. The blue, red and green lines indicate $4 \mathrm{~N}, 6 \mathrm{~N}$ and $8 \mathrm{~N}$ of static friction, respectively. . . . . . . . . . . . . . . . . 174

A.20 Robot motion results of the controlled system with varying magnitudes of dry friction interacting with the environment. The blue, red and green lines indicate $4 \mathrm{~N}, 6 \mathrm{~N}$ and $8 \mathrm{~N}$ of static friction, respectively. . . . . . . . . . . . . . . . 175

A.21 CLS deflection results of the controlled system with varying magnitudes of dry friction impacting a rigid environment. The blue, red and green lines indicate $4 \mathrm{~N}, 6 \mathrm{~N}$ and $8 \mathrm{~N}$ of static friction, respectively. ........................... 17

A.22 Robot motion results of the controlled system with varying magnitudes of dry friction impacting a rigid environment. The blue, red and green lines indicate $4 \mathrm{~N}, 6 \mathrm{~N}$ and $8 \mathrm{~N}$ of static friction, respectively. . . . . . . . . . . . . . . . 176 
A.23 Contact force results of the controlled system with varying magnitudes of dry friction impacting the head model. The blue, red and green lines indicate $4 \mathrm{~N}, 6 \mathrm{~N}$ and $8 \mathrm{~N}$ of static friction, respectively. 177 


\section{Nomenclature}

Throughout the thesis, the following nomenclature and acronyms are employed:

SOIS Safety-Oriented Interaction Strategy

HRI Human-Robot Interaction

CLS Compliant Limb Sensor

ViGRR Virtual Gait Rehabilitation Robot

DoF Degree of freedom

$\vec{x}_{d} \quad$ Deformations of the compliant limb sensor

$\vec{x}_{R}$ Pose of the point of connection of the compliant limb sensor to the robot in the world frame

$\vec{x}_{S} \quad$ Pose of the external shell of the compliant limb sensor in the world frame

$\vec{x}_{\text {base }}$ Pose of the point of connection of the compliant limb sensor to the robot with respect to the external shell of the compliant limb sensor

$\vec{x}_{d e s}$ Desired position of the position control law

$X_{R}(s)$ Laplace transform of the robot position for the single degree of freedom linear time invariant robot 
$X_{S}(s) \quad$ Laplace transform of the external shell position for the single degree of freedom linear time invariant compliant limb sensor

$X_{d}(s) \quad$ Laplace transform of the compliant limb sensor deformation for the single degree of freedom linear time invariant compliant limb sensor

$F_{\text {ext }}$ Externally applied forces applied to the single degree of freedom linear time invariant compliant limb sensor

$F_{\text {act }}$ Actuator force of the single degree of freedom linear time invariant robot

$F_{\text {sat }}$ Saturation force for the single degree of freedom actuator

$\vec{q} \quad$ Joint positions of the potentiometer based compliant limb sensor

$Z(s)$ Laplace transform of the perceived impedance of the robot from the perspective of the environment

$Z_{d} \quad$ Minimum impedance required to cause injury

$K_{S} \quad$ Stiffness of the single degree of freedom linear time invariant compliant limb sensor

$B_{S} \quad$ Damping coefficient of the single degree of freedom linear time invariant compliant limb sensor

$M_{S}$ Inertia of the single degree of freedom linear time invariant compliant limb sensor

$K_{E} \quad$ Stiffness of the single degree of freedom linear time invariant environment used in simulation

$B_{E} \quad$ Damping coefficient of the single degree of freedom linear time invariant environment used in simulation

$M_{E}$ Inertia of the single degree of freedom linear time invariant environment used in simulation

$B_{R} \quad$ Stiffness of the single degree of freedom linear time invariant robot

$M_{R}$ Damping coefficient of the single degree of freedom linear time invariant robot 
$K_{C} \quad$ Stiffness of the connection between the linear compliant limb sensor and the environment

$B_{C} \quad$ Damping coefficient of the connection between the linear compliant limb sensor and the environment

$K_{p}$ Proportional control gain for the proportional-derivative position control law

$K_{d}$ Derivative control gain for the proportional-derivative position control law

$x_{d \max }$ Maximum possible deformation of the single degree of freedom compliant limb

$V_{\max }$ Maximum impact velocity without resulting in complete compression of the compliant limb sensor

$V_{\text {dmax }}$ Desired maximum impact velocity without resulting in complete compression of the compliant limb sensor for the single degree of freedom linear time invariant system

$V_{\max O p} \quad$ Specified maximum safe operating velocity of the robot

$\Delta x_{R} \quad$ Steady state offset during free motion of the single degree of freedom linear time invariant robot

$\Delta x_{\text {Rmax }}$ Maximum allowable of the steady state offset during free motion of the single degree of freedom linear time invariant robot

$P O_{\max }$ Maximum allowable percent overshoot of the single degree of freedom, linear time invariant robot 


\section{Chapter 1}

\section{Introduction}

Robotic systems are becoming ubiquitous not only in manufacturing but in many new areas of application. The International Federation of Robotics estimates that the total number of operational industrial robots worldwide was between 1.4 and 1.6 million units by the end of 2013 with the number of service robots sold in the same year being approximately 4 million [4]. Robots are being developed to assist in the home with examples including sophisticated robots such as the Robutler that can serve beverages [5], as well as simple examples such as the popular automatic vacuum cleaning robot the Roomba [6]. Robots are designed for surveillance in home security [7], as dance partners for recreation [8] and in the medical field, assisting in rehabilitation $[9,10]$ and surgery [11]. Robots are even called upon to provide companionship to users $[12,13]$.

With the expanding applications of robotics, robots are required to interact with people in unstructured environments. In performing Human Robot Interaction (HRI), a key concern is ensuring the user's safety [14]. Robots can impose risk of physical injury to users through unexpected collisions, pinching and clamping, among other possible injury mechanisms. This risk may be exacerbated in 
the case of medical robotics where fragile users may be more susceptible to injuries [15]. Safety measures must be designed to prevent injury during HRI.

\subsection{Motivation}

While causing some safety concerns, HRI applications can still provide many benefits to their field. Interactive robots can benefit various applications in the following ways:

\section{Automation}

With the advent of HRI, many tasks that were required to be performed around people such as house cleaning [6], can now be automated using robotics. This automation is a driving force behind the development of medical robotics and robotics for personal care [16]. With the ageing population rapidly increasing, automation of care and treatment may become vital for supporting this demographic.

\section{Extension of human capabilities}

Robotic systems with HRI are able to perform tasks that people are unable to do otherwise. An example of this is the use of robots in microsurgery to allow surgeons to accurately perform tasks on a small scale [17].

\section{Increased Efficiency}

Together, humans and robots can accomplish tasks with greater efficiency than either can separately. This efficiency provides motivation for the introduction of human robot co-manufacturing as human-robot work cells would be able to perform assembly tasks normally requiring separate human and robot 
workspaces [18].

\section{Improved Accuracy, Repeatability and Sensing}

Robots have the benefits of high positioning accuracy, high repeatability and a wealth of sensors to record relevant information. HRI allows these benefits to be extended to tasks normally restricted to human workers. An example is rehabilitation robotics where robots can provide very specific, repeatable therapy with sensor measurements to monitor the patient's progress [19].

\section{Safety}

While the introduction of robots to environments involving humans poses inherent risk, robots have the possibility of removing the need for people to perform dangerous tasks. An obvious example of increased safety is the use of rescue robots [20]; however, robots can enhance safety in many other fields. Gait rehabilitation robots such as GaitEnable, for stroke patients remove the need for therapists to support a patient during gait therapy, reducing the risk of falls injuring practitioners [10].

The aforementioned advantages for HRI has spurred research efforts to ensure the safety of the user. While such research has produced insight into the factors affecting injury in robot-human collisions and some mechanisms to prevent such injuries, many robotic systems still lack a means of ensuring safety. The current research seeks to develop a Safety-Oriented Interaction Strategy (SOIS) to ensure user safety for a lower limb rehabilitation robot. The SOIS seeks to implement a tactile based collision detection and reaction strategy to prevent injury should collisions occur. 


\subsection{Background}

The current work touches upon and draws from many aspects of human robot interaction including the factors affecting injuries in HRI, safety mechanisms to prevent injury, force control methodologies and tactile sensing. This section seeks to establish the circumstances in which the current research is conducted, and provides a brief history of the larger research project to which the work is applied.

\subsubsection{Literature Review}

\subsubsection{Factors Affecting Injury in Robot-Human Collisions}

To ensure safety in HRI, robotics engineers have sought measures to predict injury during collision. An early approach borrowed danger indices developed in the automotive industry, the most popular of which being the head injury criterion (HIC) $[21,22]$. Unfortunately, injury metrics from the automotive industry were later found to be a poor indicator of danger in robotics due to unconstrained collision due to the low speeds of collision in comparison to car accidents [23,24]. Injury metrics need to be tested and proposed for the specific risks posed by robots.

Without proper metrics available, numerous safety measures have been proposed in the literature for various types of injury, a brief summary of which can be found in [25]. In [14], the impulse force generated during collision is proposed as a metric to compare the severities of impact. Ikuta et al. recognized the need for multiple metrics to address various types of injuries including applied force and stress, and proposed a unified method to evaluate safety measures by considering 
these various risks [16]. Yamada et al. employed contact forces as a metric to predict pain, important for physical and psychological well being of users [26]. Yamada supported the use of force for inferring pain with impact tests to determine the threshold force for pain at various locations on the body. Additionally, Povse et al. proposed the use of strain energy density of human skin to measure thresholds for pain [27-29]. Impact tests were also performed and the reported pain intensity was correlated with the strain energy density due to impact. For soft tissue injuries, Park et al. proposed the use of strain energy density and maximum tensile stress to estimate the severity of contusions and lacerations, respectively [30]. This study developed a model to predict these quantities based on the robot and impact parameters. In [31], stress is also proposed as a general metric to predict injury in the skin, and another model is developed to estimate the maximum stress during an impact. With the exception of the work in [26-29], the above indicators of injury were based on existing biomechanics knowledge.

A large contribution to the understanding of injury due to human robot collisions stems from the work of Haddadin et al. [1, 24,32-35]. Throughout the extensive body of work, a thorough analysis of the types of injuries during unexpected collisions is presented, see Fig. 1.1. In [24], the first systematic evaluation of robot safety using standard crash testing was performed for the DLR LWRIII, a light weight manipulator with joint torque sensors. The evaluation demonstrated the inability of severity indices from automotive testing to be applied to HRI, as all indicators suggested very low probability of serious injury while impact forces still reached values of $2 \mathrm{kN}$-a force capable of crushing a human skull. Crash testing analysis was extended to multiple industrial manipulators for constrained and 


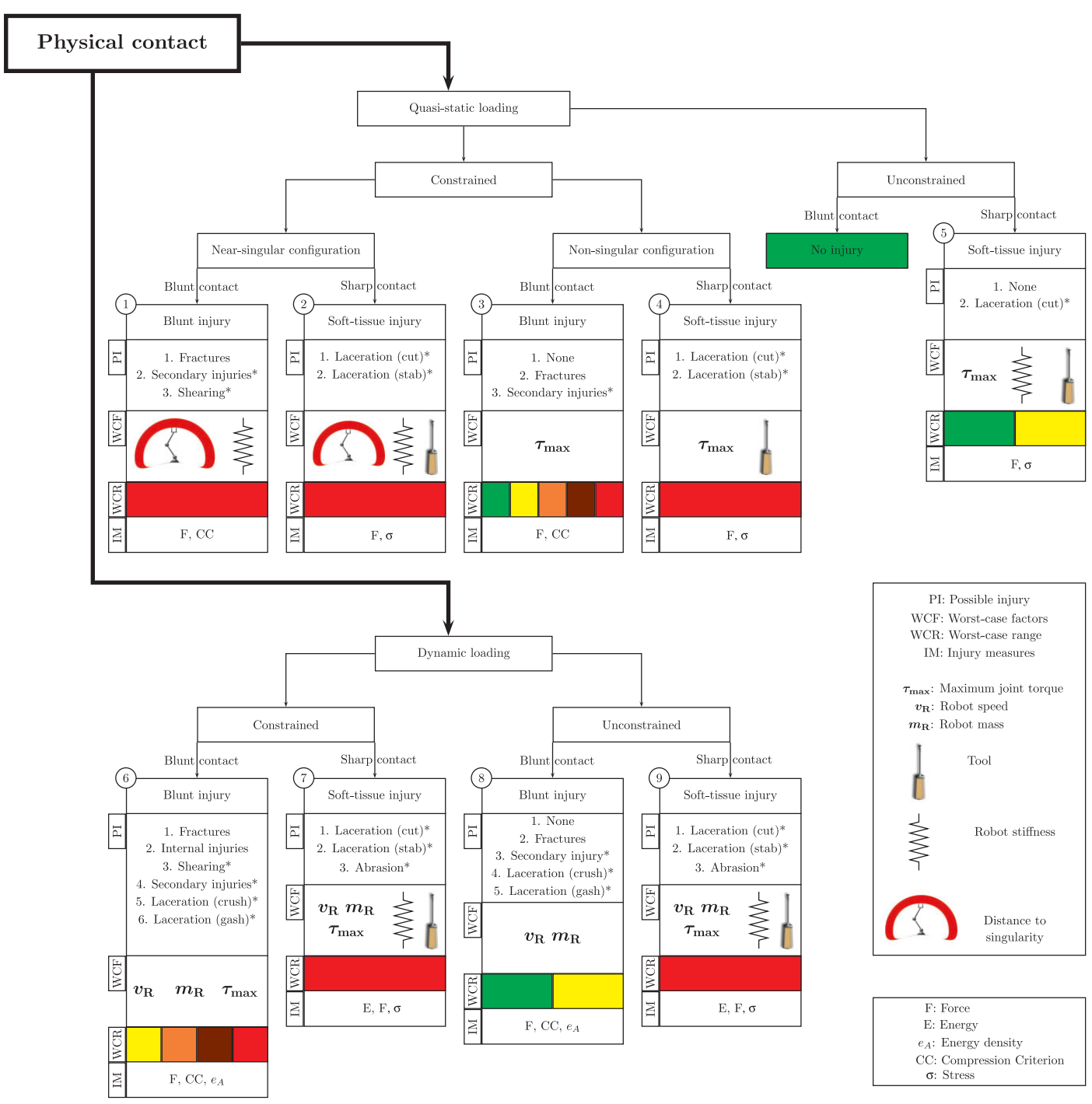

\section{Figure 1.1: Description of possible injuries due to physical contact between a human and robot [1].}

unconstrained impacts in $[32,33]$. It was found that while severity indices from the automotive industry indicated very low probability of serious injury during unconstrained collision, the impact force could cause fracture of cranial bones. For constrained collisions, large contact forces generated by continued compression after impact and the compression criteria for the chest (from the automotive 
industry) indicated danger for industrial manipulators due to large braking distances. The work in $[34,35]$ examined soft tissue injuries due to sharp and blunt impact, measuring penetration and contact forces required to cause specified soft tissue injury on pig tissue.

While the aforementioned body of research proposes many different measureable quantities to predict specific forms of injury in HRI, two common elements can be observed:

1. The mass, velocity, surface topology, and compliance of the robot are key parameters in estimating injury during collision. Mass and velocity of the robot affect the immediate contact force during collision and influence the braking distance of the robot, affecting the injury of constrained impacts. The shape and compliance of the robot surface in contact with the person affects the stress and strain energy density of the skin, significantly influencing soft tissue damage. These parameters were recommended to be mapped directly to injury for the case of soft tissue injury [35].

2. All injury metrics are related to impact force. Whether the exact injury corresponds best with acceleration, force, strain energy density, or maximum stress, the contact forces between the human and robot affect the metric and so is related to severity of injury. 


\subsubsection{Existing Approaches to Robot Safety}

Traditional robot safety measures employed in industry sought to prevent interaction between the user and robot, using mechanical barriers or sensors to determine when a user entered the robot workspace [36,37]. Such safety measures are not suitable for HRI and require the development of further measures. As unexpected collisions are a major source of injury, a variety of collision avoidance schemes have been proposed [38-40]; however, the reliability of such measures is questioned in $[14,21,41]$ and injury cannot be accepted in the case of failure in collision avoidance. Therefore, more reliable measures that seek to mitigate injury even if a collision occurs are required. In the literature various approaches have been taken to ensure safety should a collision occur. These methods are:

Ensuring safety through mechanical design: One approach to ensuring safety involves employing mechanical design and the incorporation of compliance to minimize the potential for injury in case of collisions. Lim and Tanie proposed the use of a compliant base with a light weight manipulator to deform during collision and prevent the accumulation of force [42]. Unfortunately, the method required a calculation of a new trajectory based on the measured deformation of the base and, while capable of eliminating the accumulation of force seen in constrained collision, could not affect the initial contact forces due to the robot link inertia. A mechanism was subsequently proposed to trigger during collisions to either disconnect the point of contact or to retreat. The authors of [43-45] proposed to trigger mechanisms during collision to either disconnect the point of contact or to retreat. These methods present an uncontrolled behaviour to 
the robot and can result in secondary collisions. The use of compliant actuation combined with light weight design was proposed in $[21,22,46]$. Compliant actuation decouples the colliding link from the inertia of the rest of the manipulator, resulting in decreased impact forces. However, compliant actuation reduces the control bandwidth [21] and requires robot redesign.

Incorporating safety into the robot outputs: Another approach to mitigating injury from collision is to ensure that, should an unexpected collision occur, the robot cannot injure the user. The first standard for safety in HRI, ISO-10218, employed such tactics by restricting the robot to either a maximum velocity of $0.25 \mathrm{~m} \mathrm{~s}^{-1}$, a maximum force of $150 \mathrm{~N}$ or a maximum power of $80 \mathrm{~W}$ [24]. In [14], using an equation for the potential impulsive force due to collision for a given robot velocity and pose, Heinzmann et al. proposes the use of a control law to "clip" commanded torques that would cause a potential impulse force exceeding a chosen threshold. In [35], it is proposed to limit robot motion such that the effective mass and velocity of any potentially dangerous surface of the robot cannot inflict injury. The mass and velocity required to cause injury for specific surface geometry are to be determined through biomechanics testing and it is proposed that an online database be established to store the results of such testing for use by the robotics community. While capable of being implemented on all robots, limiting the robot motion and torques to satisfy safety can be overly restricting for manipulators requiring relatively high speeds and output forces.

Collision detection and reaction: Rather than ensuring safety solely via control or mechanical design, collision detection and reaction seeks to combine 
the two strategies to ensure safety. Collision detection and reaction employs compliance to mitigate contact forces while control methods detect collision and command the robot to prevent further injury. Yamada et al. employed surface compliance to decrease impact forces below the proposed pain threshold while model based collision detection prevented the build up of forces by commanding the robot to stop $[26,47]$. The work also employed tactile sensors to detect impact prior to the model based collision detection and, through direct adjustment of the reference voltage signal, decrease the speed of the robot. In [48], two model based, high pass filtered collision detection schemes were employed to detect impact after which four strategies were employed to react (stopping, zero gravity force control, zero gravity force control with inertia shaping, and admittance control). Stopping and admittance control were found to provide the fastest response; however, zero gravity force control displayed the advantageous behaviour of yielding under subsequent contact. These methods have been shown to be effective even during soft tissue contact with a sharp tool [34]. While effective, the method presented in [34] relies on light weight manipulator design and joint torque sensing, requiring robot redesign. In addition, model based collision detection may be unsuitable for larger manipulators where collision forces may be hidden in errors in the dynamics model.

Attempting to overcome this limitation, the use of tactile sensors on the surface of the robot to detect collision and respond was proposed [2, 49]. In [49], the use of human-like reflex motions was proposed to allow the user to predict the motion of the robot in case of collision. Based on collected motion capture data [50], the authors developed a mapping between point of contact and reflex 
joint motion. However, the mapping required further experiments to mimic human motion and resulted in self collisions in the manipulator arm. Yigit et al. suggested the use of multiple reflex motions (replay, wince, yield to contact, yield to force, slow, stop, and ignore) to respond to collisions detected by tactile sensors [2]. The work proposed a decision making tree to choose between the various options in the control law considering the circumstances around the collision, see Fig. 1.2. While addressing the changing circumstances a robot is expected to face in HRI, the methodology requires selection of the robot response prior to implementation and the work did not show the effectiveness of such methods. Despite the disadvantages of the research presented above, collision detection and reaction via tactile sensors appears to be the most viable option for retrofitting existing robots for safety in HRI.

\subsubsection{Force Control Methodologies}

While commanding the robot to stop is a common response to collision in the literature, it is not the most practical choice if the user is unable to move the robot afterward [14]. An alternative approach is to control the robot to apply zero force on the environment. In this way the robot yields to contact while still preventing the subsequent build up of forces, providing users with a sense of control over the robot [48]. A variety of force control laws exist in the literature [51-53]; however, only the most common schemes, i.e. interaction, impedance control, admittance control, and outer-loop force control are only discussed, due to their relevance to HRI. 


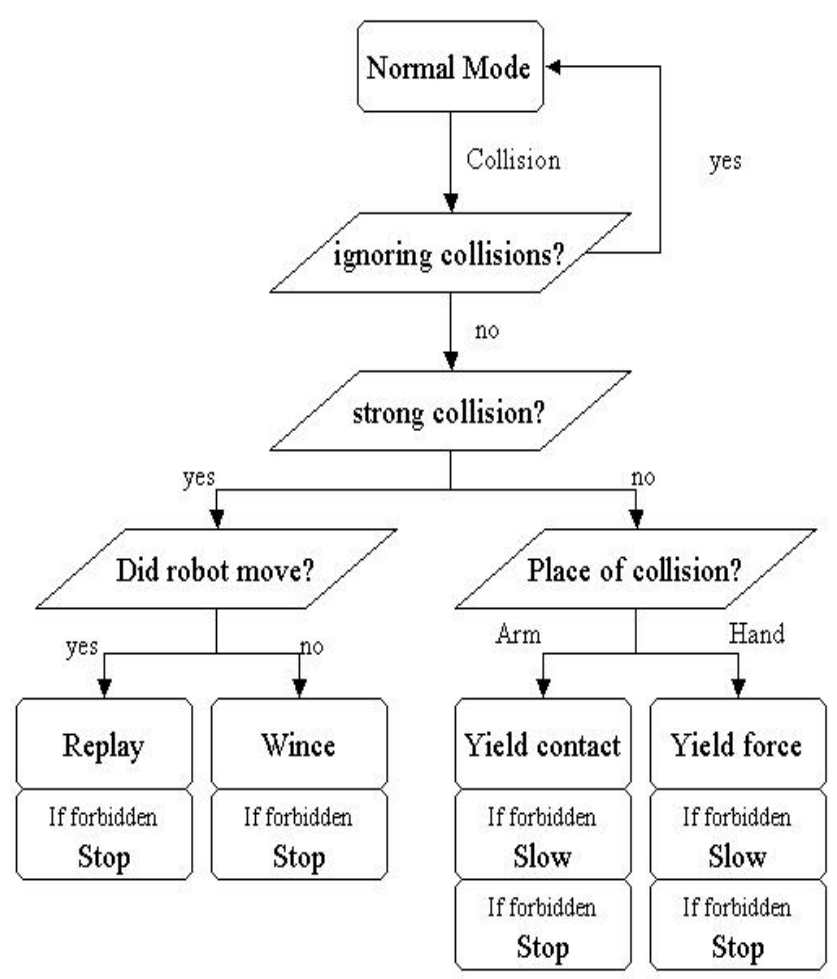

Figure 1.2: Decision tree to determine which collision reaction should be applied in a given situation [2]. 
Impedance Control: Impedance control was first proposed by Hogan as a means of applying forces to the environment without the instability issues associated with direct force feedback [54]. It is proposed that during interaction between a robot and the environment, not only must the force or position be controlled, but also the relationship between the force and position for the robot [55-58]. Two definitions of such a relationship exist, impedance and admittance. During interaction between the robot and the environment, one must behave as an impedance while the other presents admittance. Considering the presence of dynamic elements in the environment that can only be modelled as admittances, Hogan proposed the robot present an impedance through the use of the control law [54],

$$
\begin{aligned}
\tau_{a c t}= & J^{T} W^{-1} M_{I}^{-1}\left(K_{I}\left(x_{o}-x\right)-B_{I} \dot{x}\right)+J^{T} W^{-1}\left(J M^{-1} C \dot{\theta}+\dot{J} \dot{\theta}\right) \\
& +J^{T}\left(I-W^{-1} M_{I}^{-1}\right) F_{e x t}
\end{aligned}
$$

where $\tau_{a c t}$ are the motor torques, $J$ is the Jacobian of the robot, $M$ is the inertia matrix of the robot in joint space, $x$ and $x_{o}$ are the measured and desired Cartesian end effector positions, $K_{I}, B_{I}$ and $M_{I}$ are the desired end effector stiffness, damping and inertia, $W$ is the mobility tensor and $F_{\text {ext }}$ is the measured end effector force. This equation does not consider gravity in the robot model and so a gravity compensation term should be added to prevent the trajectory deviations due to the weight of the links. The above equation specifies a second order impedance model to be general and consistent with the original impedance control publications [54-58]. In many applications employing impedance control, notably in haptics research, impedance control does not attempt to vary the 
perceived mass of the manipulator [59].

Impedance control can be viewed as the use of an external position control loop that converts trajectory deviations to the desired force for a given impedance with an internal force control loop to apply the desired force [60], as seen in Fig. 1.3. While capable of changing the perceived impedance of the manipulator, impedance control has an upper limit on the stiffness it is able to render in a stable manner [60]. Errors in the robot model will also behave as external forces on the end effector [53]. These unmodelled dynamics result in poor trajectory following in free space. Despite these disadvantages, the control law does allow for stable interactions [54] and has been adapted to include more robust techniques [52], whole body force control [61] and hybrid schemes [62,63].

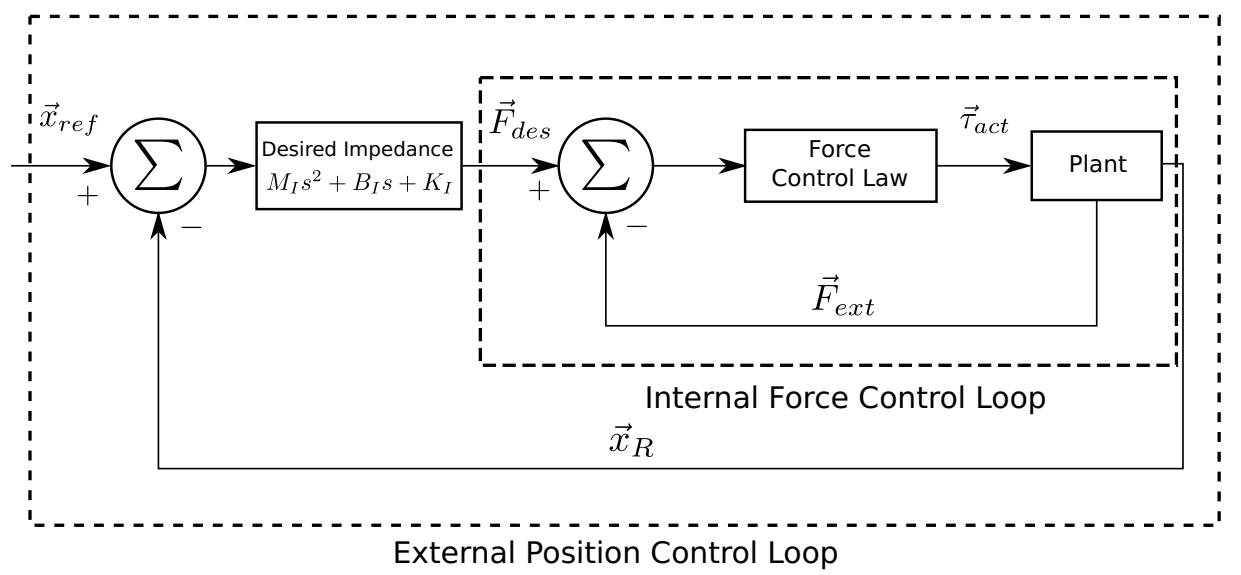

Figure 1.3: Representative block diagram of impedance control.

Admittance Control: Admittance control was first proposed by Whitney as a means to incorporate force feedback into a position controller [64]. Similar to impedance control, admittance control seeks to vary the perceived dynamics of the robot. To achieve this, admittance control generates trajectory deviations 
based on the measured deviation from a desired force [53]. Admittance control can then be viewed as an external force control loop with an internal position controller to apply trajectory deviations calculated using an admittance model (see Fig. 1.4).

Due to the internal position control law, admittance control is able to follow a trajectory accurately in free space; however, the controller can become unstable when interacting with a rigid environment [63]. Admittance control also has a minimum bound on the stiffness it is able to emulate for a given manipulator [60]. Despite these disadvantages, admittance control does not require accurate torque commands from the actuators and does not rely on the cancellation of manipulator dynamics. These features allow admittance control to be employed with large manipulators with high joint friction $[65,66]$.

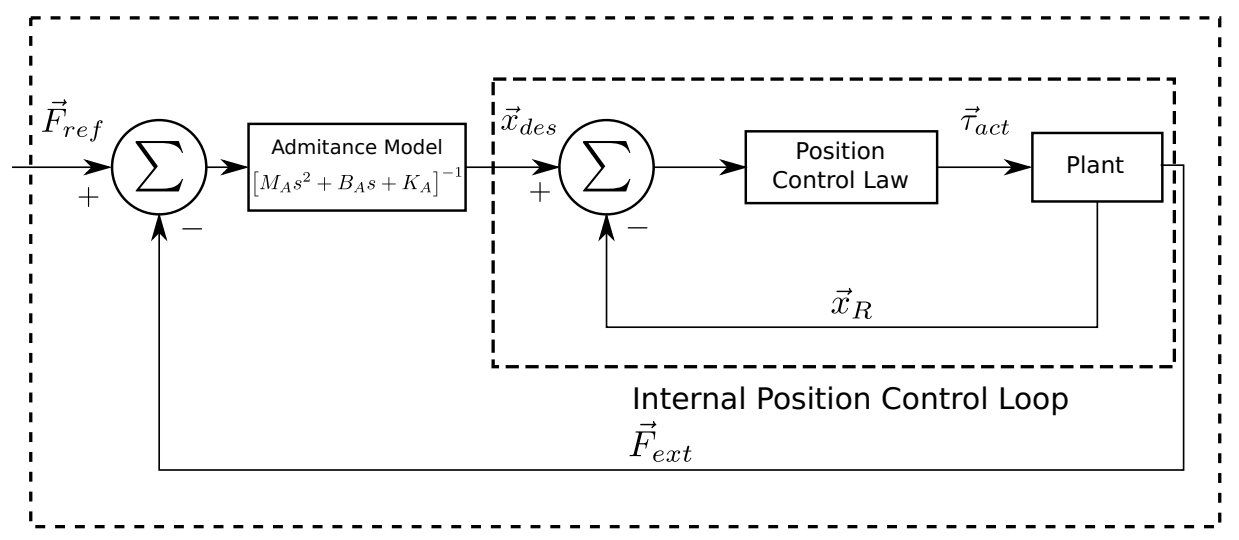

External Force Control Loop

Figure 1.4: Representative block diagram of admittance control.

Outer loop force control for compliant sensors: In comparison to impedance and admittance control, outer loop force control does not seek to establish specific dynamics for the robot, only to apply a specified force. Like 
admittance control, outer loop force control generates position commands based on deviation from a desired force [67]. Such an approach has been applied previously to HRI for use with rehabilitation robotics [68]. Recent work by Lange et al. proposed a revised force control in which the trajectory deviations were calculated using the known compliance of a compliant force sensor or the compliance of the combined robot and environment $[69,70]$. In the controller, the robot is commanded to move to the location to produce the deformations required to apply the desired force. The stability of the control law with respect to time delays was established for a position control law with linear dynamics and a force sensor modelled as a spring. The control law demonstrated improved transient response over traditional position based force control and the controller was patented [71]. Despite the advantages of this method, the use of a compliant sensor on the end effector of the robot causes difficulties in controlling the end effector position due to motion of the sensor caused by acceleration [72]. The method can be applied without a compliant sensor; however, the compliance of the robot joints and environment must be known through calibration [70]. In addition, the specific control law has been employed only in industrial applications and not for HRI.

\subsubsection{State of the Art in Tactile Sensing}

To allow collision detection for robots without the use of joint torque sensors or model based disturbance estimators, tactile sensors are required along the length of the manipulator. Reviews of tactile sensing technology for robotics are provided in $[73,74]$. In the tactile sensor literature, efforts have been made to improve safety in HRI by employing tactile sensors for collision detection and reaction, as the SOIS seeks to do. Safety in HRI using tactile sensors was explored in the following 
publications:

Combining haptic sensing with safe interaction [75]: Battaglia et al. developed a layered tactile sensor consisting of a compliant surface overlaying an array of contact switches mounted on a force sensing layer. The contact switches were employed in a keyboard matrix to reduce wiring. Several studies were performed to determine the effect of the compliant covering on the detection of contact using the switches, with the trend that thicker coverings resulted in less sensitivity. The sensor was tested on a single degree of freedom platform. The sensor had the advantage of being a low cost solution, with minimal wiring and modularity. However, there was low sensitivity of the contact switches and the multiple layers increased the mass of the sensor.

\section{A flexible robot skin for safe physical human robot interaction [76]:}

Duchaine et al. designed a compliant tactile sensor array to ensure safety in HRI. The sensor consisted of two layers of flexible conductive material separated by a compliant force sensitive resistor. The sensor was coated in polymer to improve durability and metal balls were placed on the underside of the sensor to act as stress concentrations to improve sensitivity. When pressed, the compliant material becomes conductive and acted as a switch to indicate contact. Multiplexing circuitry and analog encoding of the digital switches reduced the number of wires and sampling time. The sensor was tested on a robot to illustrate its ability to detect and respond to collision. The sensor has the benefit of being cost effective with very minimal wiring but it fails to measure force and has significant deviations in sensitivity. 
The DLR Artificial Skin [77,78]: Strohayr et al. developed a polymer based tactile sensor array wherein the mechanical structure of the sensor placed the sensing element above the compliant covering and created stress concentrations at the sensing elements to increase sensitivity. The sensor was tested and is able to withstand impact and to detect the minuscule collision forces between a robot and a sheet of paper. This sensor has a number of advantages, including high sensitivity and high spatial resolution. However, there are difficulties in scaling the sensor to a full manipulator.

Human-robot interaction based on a sensitive bumper skin [79]: The developed tactile sensor consisted of an external metal bumper connected to the robot base via compliant material with embedded force sensors to measure normal force and optical sensors to measure lateral movement. A method to respond to collision by moving in the direction of applied force was proposed, with the speed of the robot determined by the force and limited to specified values. The control concept was implemented and shown to yield to collision when detected, though the controller simply retreated from contact and did not interact. The sensor has the advantage of using a small number of outputs, is self calibrating and has a low cost. The sensor however does not specify its compliance and the accuracy is unknown.

The above tactile sensors for safety in HRI all display atleast one of two common themes:

- The use of tactile arrays to measure contact forces at multiple locations along the robot surface; 
- The emphasis on the detection of normal forces.

The use of tactile sensor arrays allows the detection of the exact location of applied contact forces; however, the delays due to data acquisition limits the use of such arrays for collision detection where high sampling rates are required. The wiring of such arrays also poses practical implementation issues [80]. The emphasis on the detection of normal forces limits the ability of the robot to interact with the environment as the robot lacks the ability to respond in the direction of shear.

\subsubsection{Project History}

\subsubsection{The Virtual Gait Rehabilitation Robot (ViGRR)}

The current research falls under a larger research effort to develop the Virtual Gait Rehabilitation Robot (ViGRR), see Fig. 1.5. The goal of the ViGRR is to offer a variety of lower extremity rehabilitation exercises through the use of a serial manipulator connected to the patients foot [66]. While capable of simple exercises such as leg presses and treadmill training, the ViGRR also seeks to offer a virtual reality gaming environment with haptic feedback [3]. Such an environment can engage the patient for more effective therapy. The robot would also offer sensor data characterizing the therapy, assisting in monitoring the progress of the patient [81].

The current ViGRR prototype consists of a 4 rotational degree of freedom planar manipulator with the peak velocity and torque of each joint actuator specified in Table 1.1. The ViGRR prototype was restricted to planar motion for simplicity, though the ViGRR goal involves 3 dimensional motion of the end effector. 


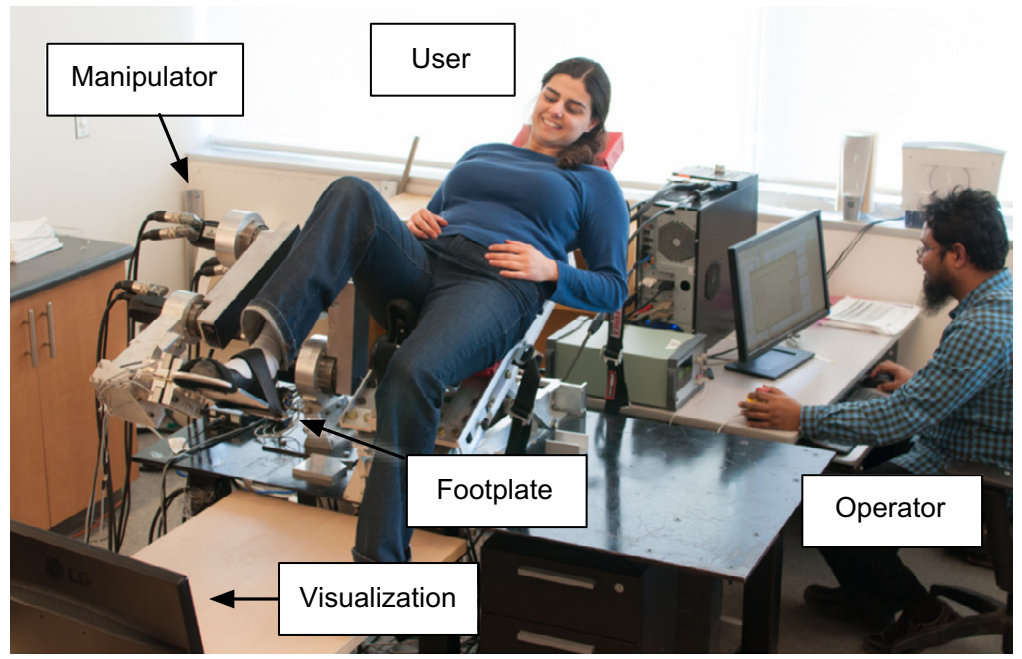

Figure 1.5: The ViGRR performing a rehabilitation exercise [3].

Table 1.1: Peak joint parameters of the ViGRR prototype.

\begin{tabular}{|l|l|l|}
\hline Joint & $\tau_{p}$ & $\omega_{p}$ \\
\hline 1 & $892 \mathrm{~N} \mathrm{~m}$ & $5.24 \mathrm{rad} \mathrm{s}^{-1}$ \\
\hline 2 & $892 \mathrm{~N} \mathrm{~m}$ & $5.24 \mathrm{rad} \mathrm{s}^{-1}$ \\
\hline 3 & $309 \mathrm{~N} \mathrm{~m}$ & $4.91 \mathrm{rad} \mathrm{s}^{-1}$ \\
\hline 4 & $191 \mathrm{~N} \mathrm{~m}$ & $8.38 \mathrm{rad} \mathrm{s}^{-1}$ \\
\hline
\end{tabular}

The redundancy of the manipulator allows the minimization of joint velocities by employing the manipulator null space. The large torques and velocities of each joint are required to mimic ground reaction forces. The transmission for each actuator is a harmonic drive that adds compliance into the robot joints. The current manipulator employs a joint level position control law and an admittance controller to apply desired forces. The haptic rendering is performed via a virtual coupling to a dynamic simulation of the virtual environment with the resulting forces applied using the admittance controller [81].

The ViGRR system is a high performance serial manipulator designed for use 
in HRI and so poses an inherent threat to users. While the joints are compliant, the manipulator is formed of large aluminium links and so, even decoupled, the links pose a threat in the case of unexpected collision. The large joint torques can also result in significant injury in case of clamping or pinching of the user in the robot. The only safety measures applied to ViGRR are the setting of joint torque limits, the use of emergency stops and a magnetic disconnect of the footplate. The current work seeks to address safety in the case of unexpected collision along the length of the robot. It is noted that while a moving guard could be used in the current case of a planar manipulator with a fixed base, the goal of ViGRR involves general motion and so a safety measure should be devised that can extend to the general case.

\subsubsection{The Compliant Limb Sensor (CLS):}

To overcome the deficits of existing collision detection methodologies for high torque robots, a new sensing method was proposed to respond to collisions for the ViGRR. The sensing method, called the Compliant Limb Sensor (CLS), consists of connecting a rigid external shell to the robot link through a compliant material. The position and orientation of the external shell are then measured to determine the deformation, $\vec{x}_{d}$, of the compliant connection, see Figure 1.6. The deformation can then be fed back to implement collision response. The CLS concept allows the separation of the compliance from the measurement method. Pose estimation of the external shell does not need to depend on the compliance connecting the shell to the robot and so, when this is true, the compliance can be varied arbitrarily. Freedom to vary the compliance allows the selection of compliance for a given application to optimally reduce contact forces considering the entire system. To 


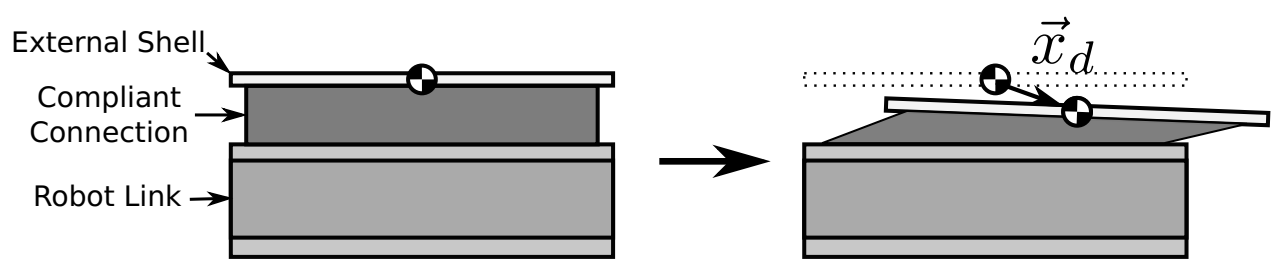

Figure 1.6: Schematic presenting the CLS concept.

detect collision, the CLS could compare $\vec{x}_{d}$ with predefined thresholds, or can employ a simple contact switch on the external surface in cases where large robot accelerations or low compliance would result in large deformations due to dynamic effects.

By covering a large area with a single hard shell, the CLS requires less sensors to cover the surface of the robot allowing for larger sampling frequencies, and requiring less wiring and data processing compared to tactile sensor arrays. The CLS also detects shear and rotation of the external shell; many tactile sensor arrays are limited to measuring compression $[73,74,76,78,80]$. The CLS also offers a simplified representation of the interaction with the environment. Whereas tactile sensor arrays present interaction as a force profile along the robot surface, the CLS represents all interaction as a small number of deformation measurements at known locations along the robot. While this representation does not provide the same amount of information as the tactile sensor array, the reduced number of inputs simplifies the feedback of the sensor information into a control law.

Preliminary work for a proof of concept prototype has been completed prior to the current research. The CLS prototype seeks to employ light-to-voltage (LTV) sensors and LEDs to estimate the external shell pose. At the time of the current research, the material for the compliant connection had been selected and the 
mechanical properties tested. A set up also exists to calibrate a model for the voltage output of the LTV sensor when stimulated by the LED.

\section{$1.3 \quad$ Objectives}

The current research aims at the development of the SOIS to prevent injury in the case of unexpected collisions between the ViGRR and the environment. The SOIS will consist of collision detection using the proposed compliant limb sensor, combined with a stable zero force control law. The CLS decouples the link inertia from the point of contact, preventing the immediate transfer of the robot kinetic energy. The zero force control law maintains the decoupling by avoiding complete CLS deflection and prevents the build up of contact forces. It is noted that, while the method is developed for the ViGRR platform, the methods presented can be extended to other areas of HRI. The specific contributions provided in the current work are:

\section{Force control law development:}

(a) Existing force control methodologies are evaluated for the current application and the use of direct deformation feedback is selected for the current work.

(b) Stability of the force control law is proven for the linear single degree of freedom case including viscous friction in the CLS when interacting with any passive environment.

(c) The ability of the control law to decrease impedance is evaluated analytically and the controller gains are found to shape the impedance 
over different, predictable frequency ranges.

(d) The ability of the control law to prevent complete CLS deformation is evaluated and shown to be directly related to the control gains. An expression to specify the maximum velocity without complete CLS deformation is determined.

(e) The effects of dry friction, sensor noise, actuator saturation and varying sampling frequencies are evaluated via simulation for the single degree of freedom case.

(f) The force control law is implemented and evaluated on a single degree of freedom manipulator.

\section{Compliant limb sensor development:}

(a) A potentiometer based compliant limb sensor is prototyped to assist in experiments for the planar extension of the control law.

(b) An optical sensor based compliant limb sensor is developed in collaboration with other researchers as a potential transduction method for the final SOIS compliant limb sensor.

(c) The accuracy of both sensors in determining sensor deformation in the plane is developed.

\subsection{Outline of Thesis}

The thesis that follows is organized into the following chapters: 


\section{Chapter 2: The Safety Oriented Interaction Strategy Con- trol Law for the Virtual Gait Rehabilitation Robot}

A list of requirements for the force control law are developed considering the ap-

plication to rehabilitation robotics and the specific ViGRR control law. The force control laws found in the literature are compared in light of these requirements and a methodology is proposed. The stability and performance are developed for a single degree of freedom linear robot and linear compliant limb sensor.

\section{Chapter 3: Validation of the Single Degree of Freedom Control Law}

Based on the linear single degree of freedom analysis, a design methodology is developed for the control law. A single degree of freedom test platform is developed and modelled as a linear system. This linear model is employed to design a control law for the system and simulate the controller to determine the effect of various unmodelled dynamics. The controller is then evaluated on the single degree of freedom system and compared to an admittance control law.

\section{Chapter 4: Initial Development of the Three Degree of Freedom Compliant Limb Sensor}

The potentiometer based and optical sensor based compliant limb sensors are developed. The dynamics of the potentiometer based sensor are determined and the sensor accuracy is evaluated. The transduction method for the optical sensor based compliant limb sensor is developed and the accuracy of the method is 
developed.

\section{Chapter 5: Conclusions and Future Work}

The work of the thesis is summarized briefly and the next steps in the development of the SOIS are elaborated upon. 


\section{Chapter 2}

\section{The Safety Oriented Interaction}

\section{Strategy Control Law for the Virtual Gait Rehabilitation Robot}

The current chapter seeks to establish the fundamental theory behind the control law proposed for the safety oriented interaction strategy (SOIS). To develop the SOIS controller, Sec. 2.1 specifies control objectives considering the application and existing control strategy of the ViGRR. The objectives help to illustrate the challenges faced in responding to collision and clearly define the expected behaviour. Once determined, Sec. 2.2 develops the control law employed for the SOIS. The benefits and disadvantages of different control schemes using the CLS are discussed and a final control method is proposed to avoid the major disadvantages observed. In Sec. 2.3, stability and performance of the proposed controller are analysed for a single degree of freedom (1DoF) linear time invariant (LTI) system. The analysis of the 1DoF LTI system provides insight into the

behaviour of the control law, the sources of instability and methods to improve 
performance.

\subsection{Control Objectives}

In selecting control objectives for the SOIS control law, the ViGRR's application must be considered. The ViGRR is designed to be moved and placed next to a patient's bed to supply mobility training soon after stroke. ViGRR's patients are physically vulnerable and attached to the robot end effector. In addition, it cannot be assured that other individuals are not nearby due to the changing location of the therapy. Control objectives must be specified considering the changing operating environments, the connection to the patient and the safety of the individuals in the surroundings. To ensure safety during the use of ViGRR, the current control law is designed to meet the following main requirements:

\section{R1: Ensure stability of free motions and interactions with any passive environment}

Stability is a minimum requirement for all control laws, and is especially important for ViGRR where unstable movements can injure the connected patient or surrounding individuals. As the controller responds to unexpected collisions, an accurate model for the interaction does not exist as ViGRR's intended application requires an uncontrolled and changing environment. Hence, the controller must stay stable when in contact with many possible environments. The current work seeks to ensure stability during interaction with any passive environment as passive environments represent a large range of dynamics including the dynamics of the human body [82]. 


\section{R2: Allow projection of the control commands into the ViGRR's null space}

Due to the connection between the patient and ViGRR, interruption of therapy resulting in unexpected end effector movements is dangerous. For this reason, therapy should not be interrupted unless a severe impact is detected as determined

by $\vec{x}_{d}$ and $\overrightarrow{\dot{x}}_{d}$ from the CLS. In the case of minor collisions, the controller should be able to mitigate injury while continuing therapy.

As ViGRR has one joint redundancy (four joints in a plane), a one dimensional null space exists in ViGRR's transformation from joint velocities to end effector Cartesian velocities. Hence, combinations of joint velocities in this null space can move the robot structure without moving the end effector. The SOIS should be able to project the control commands into this null space. By projecting into the null space, the SOIS would be able to respond to minor collisions without interrupting the admittance controller performing the therapy.

\section{R3: Minimize commanded ViGRR motion}

In responding to collisions through the CLS, the control law will command motions to avoid contact. While minimizing contact forces, such motion will move the entire robot structure and create the possibility of a second collision. For this reason, the commanded motion of the robot structure must be kept at a minimum. While the ViGRR has a redundant number of joints, maintaining the patient's foot position and orientation while responding to a collision can require up to $6 \mathrm{DoF}$ for the planar case. Hence, all 4 degrees of freedom are already specified by maintaining the foot pose and responding to collision; no joints remain to 
minimize motion of the robot structure. In this case, to minimize motion of the robot structure requires eliminating unnecessary motion in the collision response. In responding to collision, unnecessary motion includes any motion beyond the position where the CLS is just in contact with the environment. The SOIS control law must aim to reach and maintain this position.

\section{R4: Prevent complete compression of the CLS}

During a human-robot collision, a rigid contact between the individual and the robot allows a rapid transfer of the kinetic energy creating large contact forces. The CLS prevents these forces by offering a compliant point of contact. However, if the SOIS controller does not stop the robot before the complete compression of the CLS, a rigid contact will occur. This rigid contact constitutes a second collision during which any remaining robot kinetic energy is transferred, possibly resulting in injury. The SOIS control law must be able to prevent this worst case scenario for a defined robot operating range. Mathematically, this condition is described by the inequality

$$
\max \left\|\left[\frac{x_{d 1}(t)}{x_{d 1 \max }\left(\vec{x}_{d}\right)}, \ldots, \frac{x_{d n}(t)}{x_{d n \max }\left(\vec{x}_{d}\right)}\right]^{T}\right\|_{\infty}<1, i=1 \ldots n
$$

where $n$ is the total number of degrees of freedom of the sensr, $x_{d i}$ is the measured CLS deformation along the ith degree of freedom and $x_{\text {dimax }}\left(\vec{x}_{d}\right)$ is its maximum allowed. The deformation limits for each degree of freedom are a function of $\vec{x}_{d}$ for the multidegree of freedom case. 


\section{R5: Decrease the perceived impedance of the combined robot and CLS from the perspective of the environment}

Robot safety metrics for HRI use acceleration, power, energy, force and skin strain energy density as predictors of injury during collision [1, 24, 25, 28, 32]. These metrics are all related to either the instantaneous contact forces or the integral of contact forces during collision between the robot and human. However, force is not a suitable metric to minimize as the contact forces are dependent on the environment with which the robot is colliding [83]. For this reason, it is noted that the contact force and hence the safety metrics are related to the impedance of the robot as perceived by the environment (human) defined for the linear case through

$$
F(s)=Z(s) V(s)
$$

where $F(s), Z(s)$ and $V(s)$ are the Laplace transforms of the contact force, robot impedance, and robot surface velocity, respectively. As the impedance of the robot can be defined independently from the environment, it is minimized rather than the force, an approach which is supported in force control literature $[83,84]$. Impedance is a function of frequency; therefore, to ensure minimal force during any possible collision, the impedance must be minimized at all frequencies. To minimize impedance across all frequencies the following cost function is adapted 
from literature [84]

$$
\begin{aligned}
& W=\int_{\Omega_{\min }}^{\Omega_{\max }} C(\Omega) d \Omega \\
& C(\Omega)= \begin{cases}\log \left|Z\left(j 10^{\Omega}\right)\right|-\log \left|Z_{d}\right| & \forall\left|Z\left(j 10^{\Omega}\right)\right| \geq\left|Z_{d}\right| \\
0 & \forall\left|Z\left(j 10^{\Omega}\right)\right|<\left|Z_{d}\right|\end{cases}
\end{aligned}
$$

where $\Omega$ is the base ten logarithm of angular velocity $\left(\Omega=\log _{10} \omega\right)$ and $\left|Z_{d}\right|$ (in $\mathrm{Ns} \mathrm{m}^{-1}$ ) is a defined impedance bound below which there is no significant increase in safety. The band of frequencies $\left[\Omega_{\min }, \Omega_{\max }\right]$ should be chosen based on the dynamics of the CLS and robot, and the sampling rate of the control law. As a general standard, $\Omega_{\max }$ should be less than the Nyquist frequency of the controller and $\Omega_{\min }$ should be less than the first corner frequency of $Z(s)$ when the robot is uncontrolled. By choosing the optimization bounds in this way, the complete frequency response of the robot is considered while frequencies above the limits of the controller are ignored. In literature, the logarithm of frequency and impedance was chosen to provide a suitable weighting of low and high frequency impedance reduction [84]. The $\left|Z_{d}\right|$ cutoff was chosen to prevent the cost function from rewarding impedances below a level required for safety. If the impedance is below $\left|Z_{d}\right|$, the impedance is not considered dangerous; consequently, (2.3) assigns zero cost to all impedances below $\left|Z_{d}\right|$. By not rewarding reduction of impedance below this threshold, the optimization will focus on decreasing impedance at the frequencies that still pose a potential risk. While the cost function (2.3) is a measure to assess the magnitude of perceived impedance, the requirement 
on the controller is to simply decrease the perceived impedance, not to provide the optimal controller to minimize the cost function. The purpose of the cost function (2.3) is to compare between viable controllers that satisfy the safety requirements. In the current work, (2.3) is employed to select controller gains from a set of allowable gains (see Sec. 3.1).

It is noted that the above control objectives alone do not ensure safety during unexpected human-robot collisions. Even with a control law which minimizes impedance, there is no guarantee that the robot will be able to respond to prevent injury. The dynamics of the robot and environment, the robot actuation and the range of sensor compliance will limit the robot's ability to minimize force and injury. To ensure safety with the proposed system, both the mechanical design of the sensor and the controller must be considered.

\subsection{Control Methodology}

Given the control objective of minimizing perceived robot impedance, a first choice for the control law of the SOIS may be a variation of an admittance or impedance controller, as these methodologies allow for the direct specification of the perceived impedance of the robot. Despite this advantage, in considering the application of these controllers with the CLS, numerous difficulties appear. As will be explained, employing a direct feedback of the measured $\vec{x}_{d}$ from the CLS

into the existing ViGRR position controller not only overcomes these difficulties but presents other advantages as well. 


\subsubsection{Deficits with admittance and impedance control us- ing the CLS}

Both impedance [54] and admittance controls require a force sensor to achieve high levels of performance. To employ the CLS as the required force sensor, a model of the dynamic behaviour of the CLS could be proposed and calibrated. The model could then convert the measured $\vec{x}_{d}$ into a force applied to the robot at the connection to the CLS. Using this approach requires an accurate model for the CLS, otherwise the controllers will suffer from inaccurate force measurement. While capable of being employed using the CLS in this way, impedance and admittance control both suffer from limitations that suggest the use of another control scheme.

\subsubsection{Impedance control on the ViGRR using the CLS}

To use impedance control with the CLS, equation (1.1) could be employed. The dynamic parameters of the robot in (1.1) should be calculated for the point of connection between the CLS and robot. The reference trajectory for the controller should be constant at the current location with gravity compensation introduced in the model. The control law could then be employed to specify the desired inertia $\left(M_{D}\right)$, damping $\left(B_{D}\right)$ and stiffness $\left(K_{D}\right)$ of the robot from the perspective of the environment. Employing an impedance controller for the SOIS in this manner would provide the following advantages:

- The impedance of the robot as perceived by the environment can be directly manipulated. This facilitates decreasing the perceived impedance to satisfy requirement $\mathrm{R} 5$. 
- The stability of impedance control laws has been studied in the literature $[59,84,85]$, assisting in satisfying requirement R1.

- The transient response of the impedance controller can be adjusted using the desired impedance to minimize overshoot and oscillations, assisting in satisfying requirement $\mathrm{R} 4$.

Despite these advantages, the use of impedance control with the ViGRR and the CLS has many deficits such as errors due to the estimation of force from $\vec{x}_{d}$. In addition, using impedance control for the SOIS on the ViGRR includes the following limitations:

- The reference trajectory will leave the CLS in contact with the environment as the reference trajectory is set after collision is detected. This contact would result in steady state force errors proportional to the desired stiffness.

- Determining force would require the calibration of a model relating the measured $\vec{x}_{d}$ to the applied force for each planar CLS on ViGRR.

- Impedance control has difficulty mitigating the effect of friction in the robot joints. As the ViGRR's harmonic drives contain significant friction, the inability to mitigate friction could result in unacceptably high perceived impedances.

- Combining the position commands of the ViGRR admittance controller with the torque commands of impedance control using the null space could prove difficult due to their different nature. Combining the two sets of commands using the null space, as required by R2, would need further analysis to determine the effect on both controllers. 
Of these limitations, the potential difficulty in combining the impedance controller's torque commands with the ViGRR's admittance control law is particularly concerning as it prevents the ability of the controller to satisfy one of the main control objectives.

\subsubsection{Admittance control on the ViGRR using the CLS}

By generating position commands, the use of an admittance control law facilitates combining the SOIS with the existing ViGRR admittance controller and so is preferred over impedance control. To apply admittance control, the force estimated by the CLS would be fed into an admittance model to generate a trajectory to respond to the contact. The desired force for the controller would be set to zero in responding to collision. For the current application, a mass-damper is most appropriate for the admittance model as it would not result in steady state forces due to the addition of a stiffness term. In addition to generating position commands, the advantages of employing admittance control include:

- The perceived impedance of the robot by the environment can be manipulated directly by adjusting the chosen admittance model. As with impedance control, the direct manipulation of impedance assists in decreasing the perceived impedance and satisfying $\mathrm{R} 5$.

- The transient response can be controlled through the admittance model damping. Increasing the damping in the proposed mass-damper admittance model would minimize any movement beyond the position where the CLS just contacts the environment, assisting in satisfying condition R3.

- The friction of the robot can be mitigated using an admittance control law. 
By commanding robot positions, the effects of friction in the robot joints would be compensated for in the design of the internal position controller.

- A method to guarantee stability has been developed for admittance control [86], assisting in satisfying requirement R1. However, the measure can significantly impact the perceived impedance with the magnitude of the effect depending on the interaction. With the perceived impedance varying throughout interaction, minimizing impedance and satisfying R5 becomes difficult and so this method should be avoided unless instability persists after tuning the admittance model.

While providing many advantages, employing an admittance control law for the SOIS presents difficulties due to the CLS and the application. These disadvantages include:

- The need to accurately model the compliance of all CLS placed on the ViGRR. To present the user with the desired admittance model, admittance control requires accurate force measurements. Models must be calibrated for each CLS to generate forces from the measured $\vec{x}_{d}$. If the CLS is not accurately modelled and generates incorrect force measurements, the admittance controller's performance and stability may suffer.

- The chosen admittance model must be tuned for each link to ensure stability. Admittance control laws become unstable depending on the inertia, damping and stiffness of the admittance model $[59,60,85]$. To ensure stability while minimizing impedance, an admittance model should be tuned for each link. 
- The complexity of admittance control may quickly increase in multi-contact scenarios. Admittance control introduces new energy states through the admittance model. In the case of multiple contacts, more complicated admittance models must be employed [87], generating many new energy states that increase the overall system complexity.

The reliance on the accuracy of the compliance model of the CLS and the need to tune the admittance models for all points of contact greatly impedes the implementation of an admittance control law for the SOIS. In addition, the increased complexity of the controller for multi-contact scenarios generates concerns due the increased opportunity for instability in the new energy states.

\subsubsection{Proposed SOIS Control Law}

The disadvantages of admittance control tend to stem from the continued reliance on the accuracy of a compliance model to generate forces from the measured $\vec{x}_{d}$ and an admittance model to generate the position deviations $\vec{x}_{d e s}$ from the estimated forces. It is noted that both models are connected in series and serve to convert positions to forces that are converted back to positions (see Figure 2.1a). These conversions add unnecessary complexity and inaccuracy to the control law. For this reason, the current work proposes to directly feed back $\vec{x}_{d}$ to generate $\vec{x}_{d e s}$ without the use of compliance or admittance models (see Figure 2.1b). The proposed controller would then have a similar structure as an admittance control law, with an internal position control loop guided by an external trajectory generator. To replace both the compliance and admittance models, the method by which $\vec{x}_{d}$ generates $\vec{x}_{d e s}$ must: 


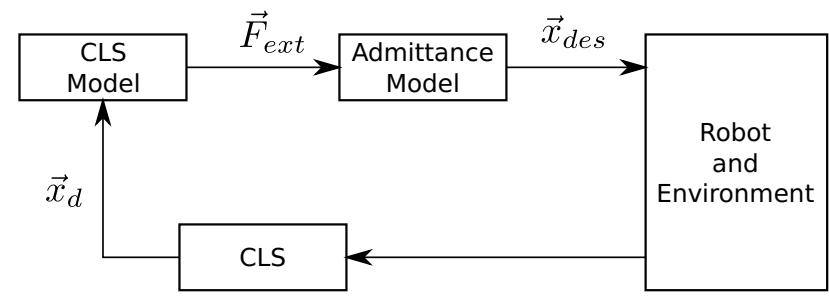

(a)

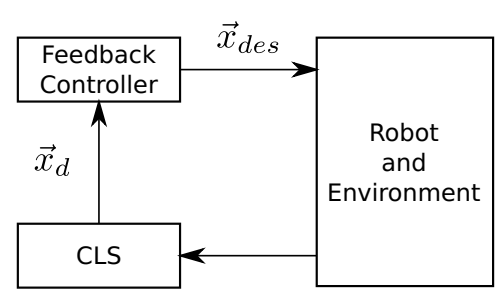

(b)

Figure 2.1: Simplified block diagrams for the generation of $\vec{x}_{d e s}$ in (a) admittance control and (b) the proposed direct feedback.

- Produce zero force at steady state $\left(\lim _{t \rightarrow \infty} F_{\text {ext }}=0\right)$. The massdamper admittance model that was proposed for the SOIS produces zero force at steady state. To replace the compliance and admittance models, the manner $\vec{x}_{d e s}$ is generated must also produce zero external force $\left(F_{\text {ext }}\right)$ at steady state.

- Produce a well defined impedance. The admittance model in an admittance control law responds in a predictable manner to the environment, allowing an impedance to be defined from the perspective of the environment $(Z(s))$. To minimize (2.3) and satisfy requirement $\mathrm{R} 5$, the generation of $\vec{x}_{d e s}$ using $\vec{x}_{d}$ must also allow the characterization of an impedance from the perspective of the environment.

One method of generating $\vec{x}_{d e s}$ using $\vec{x}_{d}$ that satisfies the above requirements would be to suspend the current robot trajectory and specify

$$
\vec{x}_{d e s}=\vec{x}_{R}-\vec{x}_{d},
$$




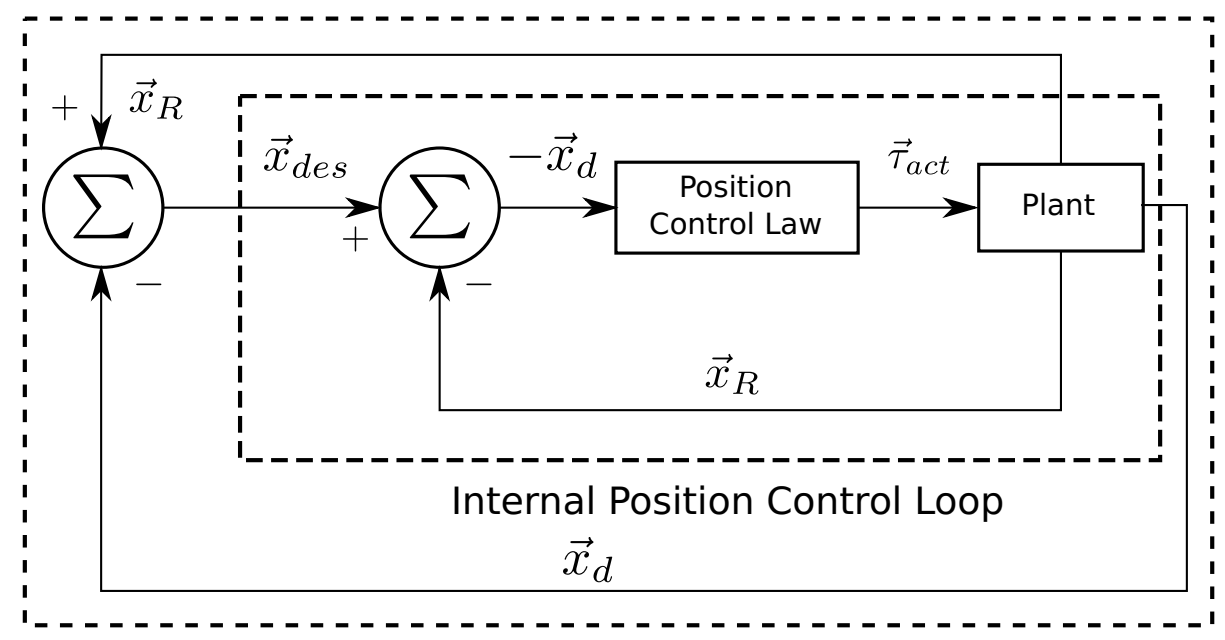

External Deformation Feedback

Figure 2.2: The block diagram of the SOIS controller for the ViGRR.

where $\vec{x}_{R}$ is the current robot position and $\vec{x}_{d}$ is positive in compression. The controller is shown in Fig. 2.2. The controller is an example of an outer loop force controller such as those found in [67-70] and constitutes a zero force control implementation of the controller in $[69,70]$. This feedback is equivalent to commanding the robot to the position where $\vec{x}_{d}$ is zero (i.e. where the CLS is just in contact with the environment). Equation (2.4) generates zero steady state force as the CLS deformation $\vec{x}_{d}$ will be zero. In addition, assuming the ViGRR's position controller's performance is known, the movement of the ViGRR due to the commanded $\vec{x}_{d e s}$ can be determined. Knowing the movements of the robot and an approximation of the CLS compliance, a perceived impedance $(Z(s))$ of the robot can be defined. While specifying $\vec{x}_{d e s}$ in this way still requires an estimate of the CLS compliance, it is noted that the CLS compliance model is only employed when minimizing $Z(s)$ to satisfy R5. The accuracy of the CLS compliance model does not affect the performance while implementing the control law. 
In addition to the improved simplicity compared to admittance control, the above feedback of $\vec{x}_{d}$ provides many attractive features that encourages its use for the SOIS, including:

- The motion commanded is kept to the minimum required to produce zero force based on the current sensor measurement by commanding the robot to the position where the CLS is believed to just be in contact with the environment. Commanding positions in this way, while employing a well damped position controller, decreases unnecessary motion in the collision response, assisting in meeting requirement R3.

- The projection of the generated position commands into the null space of the ViGRR admittance controller is simpler than with torque commands, assisting in satisfying requirement $\mathrm{R} 2$.

- The control law, like admittance control, does not inherently require modification of the position controller to be implemented (see Figure 2.2), though the perceived impedance would depend on the position control law. This is a potential advantage for the SOIS as the ViGRR position controller would not need to be changed when projecting the commands into the null space to mitigate minor collisions so long as the ViGRR controller meets any required stability conditions on the position control law.

Unfortunately, the controller does require the range of motion of the CLS to be larger than the resolution of the position control law. If the range of motion of the CLS is too small to be accurately fed back into the position controller, the control law will fail. In such cases, the CLS must be treated as a rigid force sensor with 
other force control methodologies applied. To prevent such difficulties, the CLS range of motion should be designed with the robot position control resolution in mind. Despite this disadvantage, considering the advantages of the control law and the deficits of impedance and admittance control, the SOIS control law will employ the direct feedback of $\vec{x}_{d}$.

The proposed SOIS controller constitutes a zero force case of the revised force controller specified in $[69,70]$ and is similar to the force control law described in $[67,68]$. These applications explored the stability of this feedback in the face of sampling effects and time delays assuming the force sensor was a spring. The method was applied to industrial applications and to guide therapy with an upper limb rehabilitation robot.

To the best of the author's knowledge, the stability of control law feeding back $\vec{x}_{d}$ has not been analysed with damping included in the force sensor model while the robot interacts with a general passive environment. In addition, an expression for the perceived impedance has not been found in the literature and the control law has not been applied to collision reaction along the length of the robot structure. To allow the application of the proposed SOIS control law to the ViGRR, the current research will analyse the stability and performance of the controller in regards to collision reaction when damping is included in the CLS model.

\subsection{Linear Time Invariant Controller Analysis}

The stability and performance analysis of the control law will be critical for the implementation of the SOIS on the ViGRR. While the ViGRR's dynamics are 
nonlinear with multiple degrees of freedom, the current work will gain insight into the behaviour of the control law by analysing a simpler single degree of freedom (1DoF) linear time invariant (LTI) model. This simpler system allows the use of tools available to linear systems, including the Laplace transform. The current 1DoF LTI system model cannot be used to analyse null space projection of the commands as a $1 \mathrm{DoF}$ system does not have a null space.

\subsubsection{System Model}

The proposed LTI model can be seen in Figure 2.3. Similar models were employed to investigate the stability and performance of interaction controllers in $[83,84,88]$. In the current model, the robot structure was modelled as a mass $M_{R}$ with a viscous damper $B_{R}$ to represent the friction at the joint. The CLS is modelled as a linear mass-spring-damper system with mass $M_{S}$, viscous damping $B_{S}$ and stiffness $K_{S}$. The positions of the robot and CLS masses are described by $x_{R}$ and $x_{S}$, respectively. An internal sensor measures $x_{R}$ while the CLS outputs

$$
x_{d}=x_{R}-x_{S},
$$

providing knowledge of both $x_{R}$ and $x_{S}$. Both sensors are assumed to measure without errors or time delay. The actuator is assumed to apply continuous forces without delay as dictated by the control law. The equations of motion for the current model are

$$
\begin{array}{r}
M_{R} \ddot{x}_{R}+\left(B_{R}+B_{S}\right) \dot{x}_{R}+K_{S} x_{R}=B_{S} \dot{x}_{S}+K_{S} x_{S}+F_{a c t} \\
M_{S} \ddot{x}_{S}+B_{S} \dot{x}_{S}+K_{S} x_{S}=B_{S} \dot{x}_{R}+K_{S} x_{R}+F_{\text {ext }}
\end{array}
$$


or in the Laplace domain,

$$
\begin{aligned}
{\left[M_{R} s^{2}+\left(B_{R}+B_{S}\right) s+K_{S}\right] X_{R}(s) } & =\left(B_{S} s+K_{S}\right) X_{S}(s)+F_{\text {act }}(s) \\
\left(M_{S} s^{2}+B_{S} s+K_{S}\right) X_{S}(s) & =\left(B_{S} s+K_{S}\right) X_{R}(s)+F_{\text {ext }}(s)
\end{aligned}
$$

assuming zero initial conditions. The proposed position control law to be implemented with the deformation feedback is a PD control law of the form,

$$
\begin{aligned}
F_{a c t}(s) & =\left(K_{d} s+K_{p}\right)\left(X_{\text {des }}(s)-X_{R}(s)\right) \\
& =-\left(K_{d} s+K_{p}\right) X_{d}(s) \\
& =\left(K_{d} s+K_{p}\right)\left(X_{S}(s)-X_{R}(s)\right)
\end{aligned}
$$

where $F_{a c t}(s)$ is the Laplace transform of the actuator force, $K_{d}$ and $K_{p}$ are the derivative and proportional gains of the position controller. A PD control law is employed as opposed to a general PID controller since:

- The ViGRR currently employs a PD control law.

- The desired position is the location where the CLS is undeformed. Consequently, the spring element of the CLS will not generate a steady state error that would require the use of an integral term to eliminate.

- The use of an integral term generates a second order root at zero in the Laplace transform of $x_{R}$ when the system is isolated with non zero initial conditions (see Appendix A.1 for the Laplace transform of $x_{R}$ with the PID control law). The second order root at zero will result in a nonzero steady state velocity for the robot. Steady state robot velocity is not acceptable as 
it may result in secondary collisions and may cause the robot to approach joint limits.

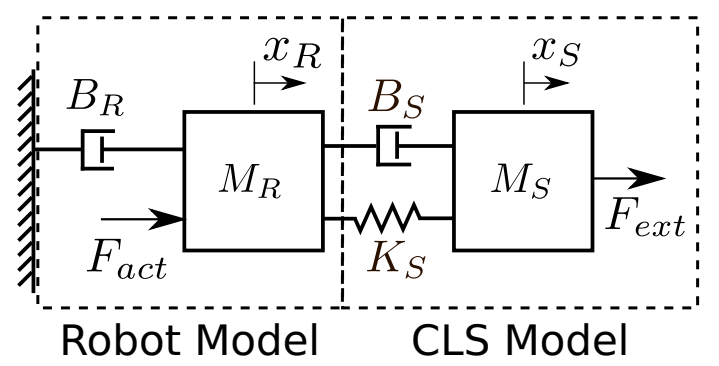

Figure 2.3: LTI model for the combined 1 DoF robot and CLS system

\subsubsection{Stability}

In analysing the stability of the proposed controller, the stability of the system without interaction (free motion stability) and the stability with interaction (interaction stability) are considered separately, as is recommended in [88]. Since the system is LTI and the controller is assumed to be continuous, both analyses are performed in the Laplace domain.

\subsubsection{Free Motion Stability}

To analyse the free motion stability of the controller, the external force is set to $0\left(F_{\text {ext }}=0 \mathrm{~N}\right)$ and non-zero initial conditions $\left(x_{R}(0), x_{S}(0), \dot{x}_{R}(0)\right.$ and $\left.\dot{x}_{S}(0)\right)$ are assumed. With these conditions, applying $X_{d}(s)=X_{R}(s)-X_{S}(s)$, (2.9) can be 
rearranged to determine the following expression for $X_{R}(s)$,

$$
X_{R}(s)=\frac{\left(M_{S} s^{2}+B_{S} s+K_{S}\right) X_{d}(s)+M_{S} s x_{S}(0)+M_{S} \dot{x}_{S}+B_{S} x_{S}(0)-B_{S} x_{R}(0)}{M_{S} s^{2}}
$$

Substituting (2.10) and (2.11) into (2.8), the Laplace transform $X_{d}(s)$ takes the form,

$$
\begin{aligned}
X_{d}(s)= & \frac{N_{1} x_{R}(0)+N_{2} x_{S}(0)+N_{3} \dot{x}_{R}(0)+N_{4} \dot{x}_{S}(0)}{D_{1} s^{3}+D_{2} s^{2}+D_{3} s+D_{4}} \\
& \text { where } \\
N_{1}= & M_{R} M_{S} s^{2}+\left(B_{R} M_{S}+B_{S} M_{R}+B_{S} M_{S}+K_{d} M_{S}\right) s+B_{R} B_{S} \\
N_{2}= & -M_{R} M_{S} s^{2}-\left(B_{R} M_{S}+B_{S} M_{R}+B_{S} M_{S}+K_{d} M_{S}\right) s-B_{R} B_{S} \\
N_{3}= & M_{R} M_{S} s+B_{R} M_{S}-B_{R} M_{S} \\
N_{4}= & -M_{R} M_{S} s-B_{R} M_{S} \\
D_{1}= & M_{R} M_{S} \\
D_{2}= & B_{R} M_{S}+B_{S} M_{R}+B_{S} M_{S}+K_{d} M_{S} \\
D_{3}= & B_{R} B_{S}+K_{p} M_{S}+K_{S} M_{S}+K_{S} M_{R} \\
D_{4}= & B_{R} K_{S} .
\end{aligned}
$$


The poles of $X_{d}(s)$ cannot become unstable. This can be shown using Routh's stability criteria, as follows

\begin{tabular}{l|ll}
$s^{3}$ & $D_{1}$ & $D_{3}$ \\
$s^{2}$ & $D_{2}$ & $D_{4}$ \\
$s^{1}$ & $E$ & 0 \\
$s^{0}$ & $E$ &
\end{tabular}

where

$$
\begin{aligned}
D_{1} & =M_{R} M_{S}>0 \\
D_{2} & =B_{R} M_{S}+B_{S} M_{R}+B_{S} M_{S}+K_{d} M_{s}>0 \\
E & =\frac{D_{2} D_{3}-D_{1} D_{4}}{D_{2}} \\
& =\frac{\left(B_{R} M_{S}+B_{s} M_{R}+B_{S} M_{S}+K_{d} M_{s}\right)\left(B_{R} B_{S}+K_{p} M_{S}+K_{S} M_{S}+K_{S} M_{R}\right)-B_{R} K_{S} M_{R} M_{S}}{B_{R} M_{S}+B_{s} M_{R}+B_{S} M_{S}+K_{d} M_{s}} \\
& \geq \frac{\left(B_{s} M_{R}+B_{S} M_{S}+K_{d} M_{s}\right)\left(B_{R} B_{S}+K_{p} M_{S}+K_{S} M_{S}\right)}{B_{R} M_{S}+B_{s} M_{R}+B_{S} M_{S}+K_{d} M_{s}}>0
\end{aligned}
$$

where all system parameters are positive and so satisfy the inequalities above. Based on the Routh-Hurwitz criterion, the value of $x_{d}$ is exponentially stable. There remains the possibility that $x_{R}$ and $x_{S}$ still become unbounded while the difference between the values converges to zero. This possibility is due to the control law not seeking to control either $x_{R}$ or $x_{S}$ directly. To check that this is 
not the case, the Laplace transforms of $x_{S}$ and $x_{R}$ were determined to be,

$$
\begin{aligned}
X_{R}(s)= & \frac{N_{1} x_{R}(0)+N_{2} x_{S}(0)+N_{3} \dot{x}_{R}(0)+N_{4} \dot{x}_{S}(0)}{D_{1} s^{4}+D_{2} s^{3}+D_{3} s^{2}+D_{4} s} \\
& \text { where } \\
N_{1}= & M_{R} M_{S} s^{3}+\left(B_{R} M_{S}+B_{S} M_{R}+B_{S} M_{S}+K_{d} M_{S}\right) s^{2} \\
& \ldots+\left(B_{R} B_{S}+K_{S} M_{R}\right) s+B_{R} K_{S}-B_{S} K_{p}+K_{d} K_{S} \\
N_{2}= & \left(K_{p} M_{S}+K_{S} M_{S}\right) s+B_{S} K_{p}-K_{d} K_{S} \\
N_{3}= & M_{R} M_{S} s^{2}+B_{S} M_{R} s+K_{S} M_{R} \\
N_{4}= & \left(B_{S} M_{S}+K_{d} M_{S}\right) s+K_{p} M_{S}+K_{S} M_{S} \\
D_{1}= & M_{R} M_{S} \\
D_{2}= & B_{R} M_{S}+B_{S} M_{R}+B_{S} M_{S}+K_{d} M_{S} \\
D_{3}= & B_{R} B_{S}+K_{p} M_{S}+K_{S} M_{S}+K_{S} M_{R} \\
D_{4}= & B_{R} K_{S}
\end{aligned}
$$




$$
\begin{aligned}
X_{S}(s)= & \frac{N_{1} x_{R}(0)+N_{2} x_{S}(0)+N_{3} \dot{x}_{R}(0)+N_{4} \dot{x}_{S}(0)}{D_{1} s^{4}+D_{2} s^{3}+D_{3} s^{3}+D_{4} s} \\
& \text { where } \\
N_{1}= & K_{S} M_{R} s+B_{R} K_{S}-B_{S} K_{p}+K_{d} K_{S} \\
N_{2}= & M_{R} M_{S} s^{3}+\left(B_{R} M_{S}+B_{S} M_{R}+B_{S} M_{S}+K_{d} M_{S}\right) s^{2} \\
& \ldots+\left(B_{R} B_{S}+K_{p} M_{S}+K_{S} M_{S}\right) s+B_{S} K_{p}-K_{d} K_{S} \\
N_{3}= & B_{S} M_{R} s+K_{S} M_{R} \\
N_{4}= & M_{R} M_{S} s^{2}+\left(B_{R} M_{S}+B_{S} M_{S}+K_{d} M_{S}\right) s+K_{p} M_{S}+K_{S} M_{S} \\
D_{1}= & M_{R} M_{S} \\
D_{2}= & B_{R} M_{S}+B_{S} M_{R}+B_{S} M_{S}+K_{d} M_{S} \\
D_{3}= & B_{R} B_{S}+K_{p} M_{S}+K_{S} M_{S}+K_{S} M_{R} \\
D_{4}= & B_{R} K_{S} .
\end{aligned}
$$

Equations (2.13) and (2.14) have the same poles as $X_{d}(s)$ with the exception of the additional pole at $s=0$. This additional pole will result in a non zero steady state response, but not unbounded behaviour. The amount of robot movement at steady state for given initial conditions is determined to be,

$$
\lim _{s \rightarrow 0} s X_{R}(s)-x_{R}(0)=\Delta x_{R}=-\frac{B_{S} K_{p}-K_{d} K_{S}}{B_{R} K_{S}} x_{d}(0)+\frac{M_{R}}{B_{R}} \dot{x}_{R}(0)+\frac{\left(K_{S}+K_{p}\right) M_{S}}{B_{R} K_{S}} \dot{x}_{S}(0) .
$$

where $\Delta x_{R}$ is the steady state motion of the robot in response to initial conditions without interaction. Based on (2.15), to decrease unwanted steady state offsets with non-zero initial conditions, the proportional gain $K_{p}$ of the controller must be decreased and the derivative gain should approach $K_{p} B_{S} / K_{S}$ to prevent offsets 
from $\dot{x}_{S}(0)$ and $x_{d}(0)$, respectively. The amount of movement due to $\dot{x}_{R}$ cannot be influenced by the current control law, most likely due to the position of the robot not being directly controlled.

In responding to collision, the control law may become disconnected from the environment due to either the person or object retreating from the point of impact or overshoot in the position control law. Such a disconnection would result in free motion behaviour with initial conditions. To meet requirement R3 and minimize commanded motion, the robot motion in response to such initial conditions must be decreased and it is recommended to do so by decreasing the steady state offsets found in (2.15), as simple conditions can be found to do so. These offsets do not account for transient oscillations but do offer some indication of the magnitude of the motion as the solution will oscillate around these offsets. If the system is reasonably damped and the CLS inertia is small, such oscillations should not affect the observed motion significantly.

\subsubsection{Interaction Stability}

The stability of the control law while interacting with a human or other unknown environment will now be investigated. The dynamics of the environment (human or otherwise) are unknown but are assumed in the current work to be passive. With this assumption, passivity is proposed as the measure of stability. Ensuring the robot is passive guarantees stability while interacting with a passive environment as the connection of two passive systems is stable [89]. The current analysis seeks to develop conditions on the $K_{p}$ and $K_{d}$ position control gains to ensure passivity when $x_{d}$ is fed back in the manner previously discussed.

A passive system is defined as a system for which the net energy transferred 
to the environment is less than the initial energy of the system [89], or

$$
\int_{0}^{t} F_{e x t}(t) V_{S}(t) d t \geq-E_{i},
$$

where $F_{\text {ext }}$ is the force applied by the environment to the system, $V_{S}$ is the velocity of the CLS mass, and $E_{i}$ is the initial energy of the system.

To analyse the current LTI system, the condition for passivity shown in equation (2.16) reduces to the requirement that the impedance of the robot as seen from the environment (i.e. $Z(s)=F_{e x t}(s) / V_{S}(s)$ ) is positive real. For a linear system with transfer function $Z(s)$ to be positive real, the following conditions must be met [89]:

1. $Z(s)$ has no poles located in the right half of the complex plane.

2. The poles of $Z(s)$ (if any) on the imaginary axis have to be simple with positive real residues.

3. $\operatorname{Re}[Z(j w)] \geq 0, \forall w \geq 0$

For the current analysis, the PD position control law used in the free motion stability analysis, (2.10), will be expressed in the form,

$$
F_{a c t}=\left(K_{d}+\frac{K_{p}}{s}\right)\left(V_{S}-V_{R}\right)
$$

where $V_{S}$ and $V_{R}$ are the CLS mass and robot velocities, respectively. To determine the impedance of the overall robotic system, (2.17) is applied to (2.8) to 
determine the following expression for $\frac{V_{R}(s)}{V_{S}(s)}$,

$$
\frac{V_{R}(s)}{V_{S}(s)}=\frac{B_{S}+K_{d}+\frac{K_{p}+K_{s}}{s}}{M_{R} s+B_{R}+K_{d}+B_{S}+\frac{K_{p}+K_{S}}{s}}
$$

Applying (2.18) to (2.9), the impedance of the overall robotic system from the perspective of the environment can be found to be

$$
Z(s)=M_{S} s+\frac{\left(B_{S} s+K_{S}\right)\left(M_{R} s+B_{R}\right)}{M_{R} s^{2}+\left(B_{R}+K_{d}+B_{S}\right) s+K_{p}+K_{S}}
$$

In evaluating the passivity of the impedance, the sum of two passive impedances is passive; consequently, the passivity of both terms in equation (2.19) can be analysed separately and then the conditions on each can be combined. The first term of (2.19) is simply the mass of the CLS and known to be passive. The second term of (2.19) represents the connection of the external shell to the underlying robot and introduces the effect of the position controller. This term includes two zeros and two poles; consequently, the conditions for positive realness need to be evaluated.

For the second term, if all parameters are greater than zero, the poles of the system will be the roots of a quadratic equation with all positive coefficients. Hence, the second term will not have poles on the imaginary axis and all poles will have negative real parts. This satisfies the first and second conditions of positive realness for the second term. The third condition of positive realness requires the real component of the second term be positive for all frequencies. 
Substituting in $s=j \omega$, this condition is found to be satisfied if,

$$
\begin{aligned}
& \operatorname{Re}[Z(j \omega)] \geq 0, \text { or } \\
& \operatorname{Re}\left[\frac{\left(B_{R} K_{S}-M_{R} B_{S} \omega^{2}+j \omega\left(M_{R} K_{S}+B_{R} B_{S}\right)\right)\left(K_{p}+K_{S}-M_{R} \omega^{2}-j \omega\left(B_{R}+B_{S}+K_{D}\right)\right)}{\left(K_{p}+K_{S}-M_{R} \omega^{2}\right)^{2}+\left(B_{R}+B_{S}+K_{D}\right)^{2}}\right] \geq 0, \text { or } \\
& a \omega^{4}+b \omega^{2}+c \geq 0
\end{aligned}
$$

where

$$
\begin{aligned}
& a=M_{R}^{2} B_{S} \\
& b=K_{d} M_{R} K_{S}-K_{p} M_{R} B_{S}+B_{R}^{2} B_{S}+B_{R} B_{S}^{2}+K_{d} B_{R} B_{S} \\
& c=B_{R} K_{S}^{2}+K_{p} B_{R} K_{S}
\end{aligned}
$$

As all parameters are positive, $a$ and $c$ are positive as well. To satisfy (2.20) when $a$ and $c$ are positive, either $b \geq 0$ or $b^{2}-4 a c \leq 0$. Condition $b \geq 0$ ensures that all terms of (2.20) are positive. Condition $b^{2}-4 a c \leq 0$ prevents (2.20) from having a zero; with $c$ positive, the cubic must remain positive for all frequencies.

If the system is found to be active, the current equation does not immediately suggest changes to satisfy passivity. In addition, the current conditions $(b \geq 0$ or $\left.b^{2}-4 a c \leq 0\right)$ require knowledge of all system parameters and do not have obvious physical interpretations. Simpler but more conservative constraints to guarantee passivity can be developed by finding conditions to ensure that $b \geq 0$. The following conditions ensure that $b \geq 0$ and so the system is passive,

$$
\begin{aligned}
& K_{d}+B_{R}+B_{S}>\frac{M_{R} K_{p}}{B_{R}} \\
& \frac{K_{S}}{B_{S}}>\frac{K_{p}}{K_{d}} .
\end{aligned}
$$


Physically, condition (2.21) implies stability can be ensured if the damping of the system is sufficiently large while condition (2.22) compares the behaviour of the spring and damper of the CLS to that of the controller. The above simplified conditions provide beneficial guide lines in interpretation and implementation. If the system becomes unstable while interacting with a passive environment, condition (2.21) indicates that one can either increase damping or decrease the $K_{p}$. With an estimate of the sensor damping and stiffness, condition (2.22) has the practical advantage of ensuring stability based solely on the controller gains.

\subsubsection{Performance}

The performance of the proposed control methodology is evaluated based on two criteria:

1. Maximum impact velocity $\left(V_{\max }\right)$ without complete compression of the CLS: For a given control law, CLS and environment, a maximum impact velocity, $V_{\max }$, can be specified such that the CLS is not completely compressed, satisfying (2.1) of R4. The larger the $V_{\max }$ for a given control law, the more resistant the controller is to complete CLS compression. If a design safety fact and the maximum operating velocity for the CLS are known, the required $V_{\max }$ can be calculated using

$$
V_{\text {max }}=F V_{\max O p}
$$

where $V_{\max O p}$ is the maximum operating velocity of the robot and $F$ is a safety factor specified by the application. 
2. The perceived impedance: The decrease in impedance provides a measure of how effectively the controller will decrease contact forces when colliding with a person. Impedance of the robotic system must be minimized to satisfy R5.

The current analysis will determine the effect of the controller on each in terms of the $K_{p}$ and $K_{d}$ controller gains for the 1DoF LTI model.

\subsubsection{Maximum Impact Velocity}

To calculate $V_{\max }$ for the direct CLS feedback, the environment is assumed to be immoveable. This assumption conservatively represents the robot impacting a stationary environment, as most environments will yield to contact and assist in preventing the CLS compression. Safety in this situation is defined as a core requirement in human-robot interaction [14].

It is noted that the environment could also be moving and assist in compressing the CLS. The assumption of a stationary environment is motivated by the fact that the environment's velocity cannot be known. In such a situation, choosing a standard velocity allows comparison between control laws. In addition, a stationary environment emphasizes the ability of the robot to dissipate and prevent the transfer of its own kinetic energy. If the user were to impact the robot at high velocity, the controller may be unable to prevent complete deformation of the CLS and the interaction would result in high impact forces. However, it is not reasonable to require the robot to prevent the complete compression of the CLS if the user impacts the robot themselves.

To determine $V_{\max }$ for the current control law, the CLS mass in Figure 2.3 is 
fixed, representing a rigid connection to an immovable environment. The Laplace transform for a fixed CLS mass with an initial robot velocity is

$$
X_{R}(s)=\frac{M_{R} \dot{x}_{R}(0)}{M_{R} s^{2}+\left(B_{S}+B_{R}+K_{d}\right) s+K_{S}+K_{p}} .
$$

$V_{\max }$ will then be the maximum $\dot{x}_{R}(0)$ that results in a deformations less than the maximum deformation of the CLS, $x_{d \max }$.

In deriving $V_{\max }, 3$ cases are considered depending on whether (2.24) is overdamped $(\zeta>1)$, critically damped $(\zeta=1)$ or underdamped $(\zeta<1)$. For the 3 cases, the $V_{\max }$ values are

$$
\begin{aligned}
& \zeta>1 \\
& V_{\max }=D x_{d \max }\left(\left(\frac{p-D / 2}{p+D / 2}\right)^{\frac{p}{D}+\frac{1}{2}}-\left(\frac{p-D / 2}{p+D / 2}\right)^{\frac{p}{D}-\frac{1}{2}}\right)^{-1} \\
& \zeta=1 \\
& V_{\text {max }}=-e p x_{d \max } \approx-2.718 p x_{d \max } \\
& \zeta<1 \\
& V_{\max }=-p x_{d \max } e^{\frac{2 p \arctan \left(\frac{D}{2 p}\right)}{D}} \sqrt{1+\left(\frac{D}{2 p}\right)^{2}}
\end{aligned}
$$

where,

$$
\begin{aligned}
p & =-\frac{B_{S}+B_{R}+K_{d}}{2 M_{R}} \\
D & =\left|\sqrt{\frac{\left(B_{S}+B_{R}+K_{d}\right)^{2}-4 M_{R}\left(K_{S}+K_{p}\right)}{M_{R}^{2}}}\right| .
\end{aligned}
$$




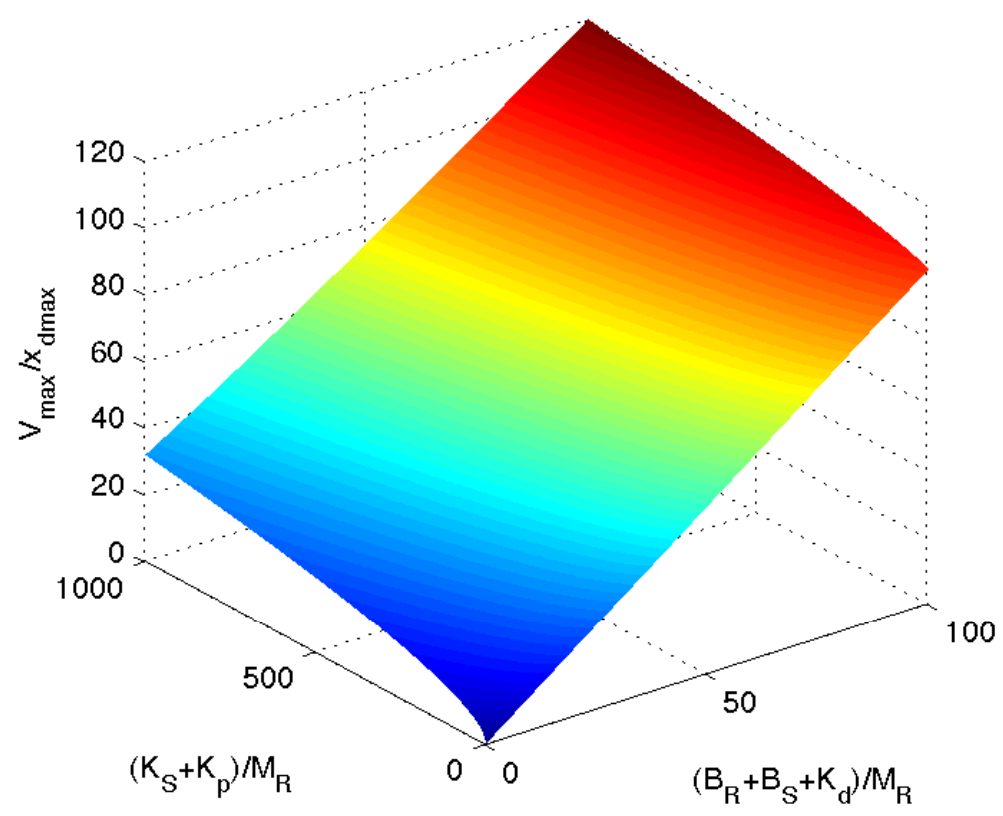

Figure 2.4: Effect of system stiffness and damping on $V_{\max }$

To investigate the relative effect of stiffness versus damping, Figure 2.4 plots $\frac{V_{\max }}{x_{d \max }}$ for system stiffness and damping normalized with respect to robot mass $\left(\frac{K_{S}+K_{p}}{M_{R}}\right.$ and $\frac{B_{R}+B_{S}+K_{d}}{M_{R}}$ respectively). From Figure 2.4, it can be seen that $V_{\text {max }}$ increases with both $\sqrt{K_{S}+K_{p}}$ and $B_{R}+B_{S}+K_{d}$. Physically, the larger effect of damping $\left(B_{R}+B_{S}+K_{d}\right)$ on $V_{\max }$ is due to the immediate response of damping to impact; the damping applies forces as soon as the CLS begins to move. In comparison, the stiffness requires motion to build up force and so cannot as effectively prevent compression of the CLS. In considering controller design, this result indicates that if $V_{\max }$ must be increased, $K_{d}$ should be varied as opposed to $K_{p}$, though increases in either will increase $V_{\max }$. 


\subsubsection{Effect on Impedance}

The effect of the control law is captured in the perceived impedance of the system (2.19). The equation (2.19) indicates that the impedance of the system is composed of two terms, the mass of the CLS (first term) and the impedance of the connection to the underlying robot (second term). The feedback of CLS deformation $x_{d}$ with a PD control law does not effect the impedance due to the mass of the CLS. The inability to influence the impedance of an inertia connected through a compliant connection is a known result, and similar results for simple linear models can be found in [83,90]. At high frequencies, the mass of the CLS

will dominate the response and create a $20 \frac{d b}{d e c a d e}$ increase in impedance. The mass of the CLS is a limiting factor in minimizing the perceived impedance and should be reduced during the design of the CLS. Conversely, the impedance of the connection to the robot is significantly impacted by the position control law, as evidenced by the controller gains in the denominator. However, the effect of the controller is still limited to low frequencies as can be seen by taking the limit,

$$
\lim _{s \rightarrow \infty} \frac{\left(B_{S} s+K_{S}\right)\left(M_{R} s+B_{R}\right)}{M_{R} s^{2}+\left(B_{R}+K_{d}+B_{S}\right) s+K_{p}+K_{S}}=\frac{M_{R} B_{S}}{M_{R}}=B_{S}
$$

Hence, the damping of the CLS will also limit the minimization of the perceived impedance at higher frequencies.

The impact of $K_{p}$ and $K_{d}$ on the impedance of the robot is illustrated in Figure 2.5-2.8. The robot parameters for the plots were the approximate inertia and damping of ViGRR's first joint $\left(M_{R}=4.2196 \mathrm{~kg}\right.$ and $\left.B_{R}=27.2051 \mathrm{~N} \mathrm{~s} \mathrm{~m}^{-1}\right)$ [66]. The CLS parameters were chosen to provide a light weight $\left(M_{S}=0.1 \mathrm{~kg}\right)$ 


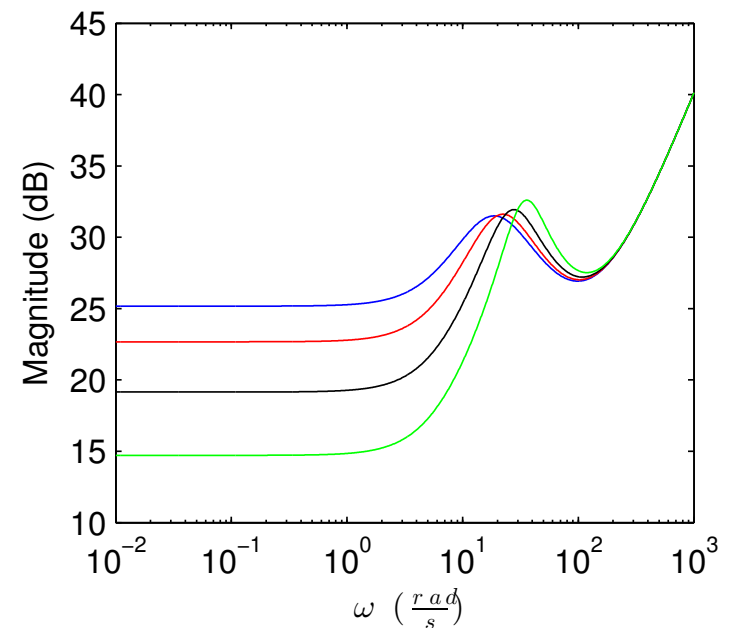

Figure 2.5: Impedance of 1DoF LTI system with $K_{d}=80 \mathrm{~N} \mathrm{sm}^{-1}$ for varying $K_{p}$ (blue $=500 \mathrm{~N} \mathrm{~m}^{-1}$, red $=1000 \mathrm{~N} \mathrm{~m}^{-1}$, black $=2000 \mathrm{~N} \mathrm{~m}^{-1}$ and green $\left.=4000 \mathrm{~N} \mathrm{~m}^{-1}\right)$.

and low stiffness $\left(K_{S}=1 \mathrm{kN} \mathrm{m}^{-1}\right)$ CLS with a critically damped behaviour $\left(B_{S}=\right.$ $20 \mathrm{~N} \mathrm{~s} \mathrm{~m}^{-1}$ ). All combinations of $K_{p}$ and $K_{d}$ gains were selected to satisfy the stability condition (2.22). In Figure 2.5-2.8, the effect of control gains on the expression,

$$
\frac{1}{M_{R} s^{2}+\left(B_{R}+B_{S}+K_{d}\right) s+K_{S}+K_{p}}
$$

is also plotted. The control gains affect the impedance through this expression and isolates the effects of the gains on the impedance.

In Fig. 2.5, increasing values of $K_{p}$ result in:

1. Decreased low frequency impedance;

2. A slight increase in the frequency and magnitude of the observed peak.

In low frequencies the response of the impedance is dominated by the low order 


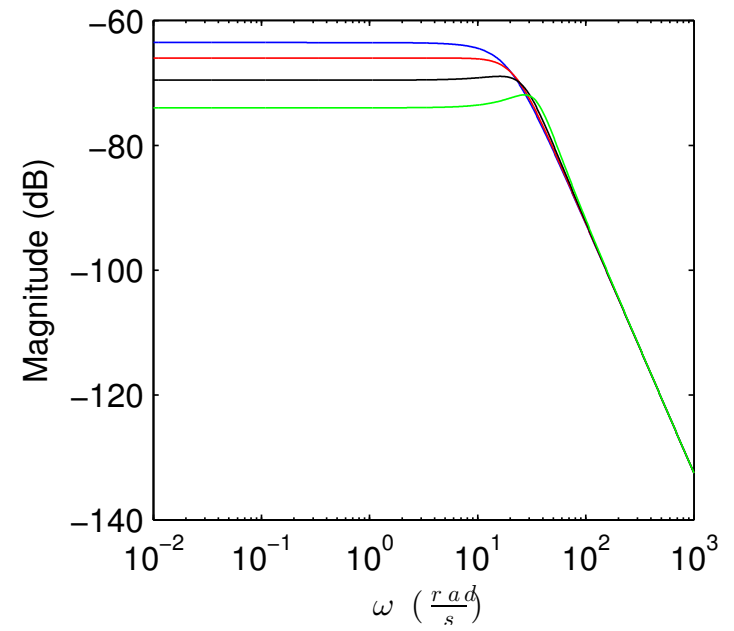

Figure 2.6: Impedance of $\left(M_{R} s^{2}+\left(B_{R}+B_{S}+K_{d}\right) s+K_{S}+K_{p}\right)^{-1}$ with $K_{d}=80 \mathrm{~N} \mathrm{~s} \mathrm{~m}^{-1}$ for varying $K_{p} \quad\left(\right.$ blue $=500 \mathrm{~N} \mathrm{~m}^{-1}$, red $=1000 \mathrm{~N} \mathrm{~m}^{-1}$, black $=2000 \mathrm{~N} \mathrm{~m}^{-1}$ and green $=4000 \mathrm{~N} \mathrm{~m}^{-1}$ ).

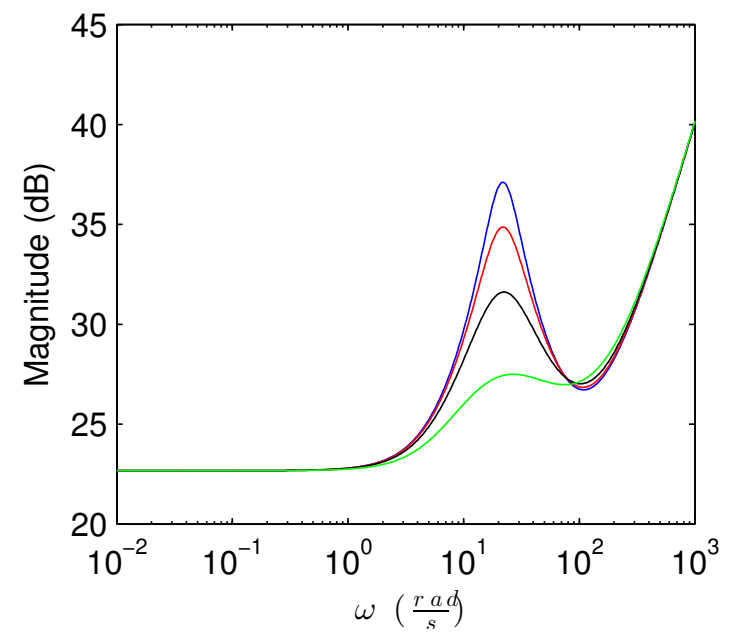

Figure 2.7: Impedance of 1DoF LTI system with $K_{p}=1000 \mathrm{Nm}^{-1}$ for varying $K_{d}$ (blue $=20 \mathrm{~N} \mathrm{~s} \mathrm{~m}^{-1}, \operatorname{red}=40 \mathrm{~N} \mathrm{~s} \mathrm{~m}^{-1}$, black $=80 \mathrm{~N} \mathrm{~s} \mathrm{~m}^{-1}$ and green $\left.=160 \mathrm{~N} \mathrm{~m}^{-1}\right)$. 


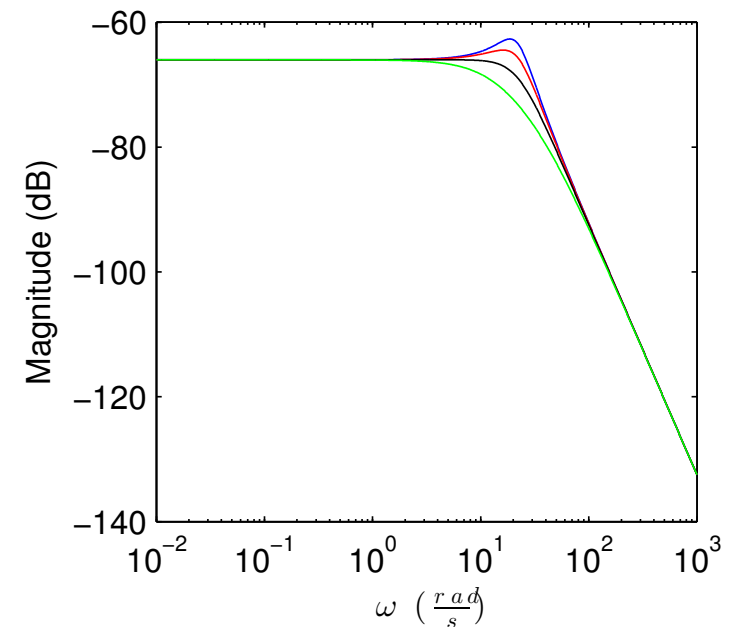

Figure 2.8: Impedance of $\left(M_{R} s^{2}+\left(B_{R}+B_{S}+K_{d}\right) s+K_{S}+K_{p}\right)^{-1}$ with $K_{p}=1000 \mathrm{~N} \mathrm{~m}^{-1}$ for varying $K_{d} \quad\left(\right.$ blue $=20 \mathrm{~N} \mathrm{~s} \mathrm{~m}^{-1}$, red $=40 \mathrm{~N} \mathrm{~s} \mathrm{~m}^{-1}$, black $=80 \mathrm{~N} \mathrm{~s} \mathrm{~m}^{-1}$ and green $=160 \mathrm{~N} \mathrm{~m}^{-1}$ ).

terms of (2.19), as is seen through the limit

$$
\lim _{s \rightarrow 0} M_{s} s+\frac{\left(B_{S} s+K_{S}\right)\left(M_{R} s+B_{R}\right)}{M_{R} s^{2}+\left(B_{R}+K_{d}+B_{S}\right) s+K_{p}+K_{S}}=\frac{K_{S} B_{R}}{K_{p}+K_{S}} .
$$

From (2.32), $K_{p}$ is inversely proportional to the low frequency impedance resulting in the marked decreases in impedance seen in Figure 2.5-2.6. By comparing Fig. 2.5 with Fig. 2.6, it is seen that the peak in impedance does not result only from the controller gain. A zero of the second term of (2.19) contributes to the rise of impedance until the poles of the second term reduce the impedance at higher frequencies, resulting in the peak. However, with increasing $K_{p}$ values, the corner frequencies of the poles increase, as seen in Fig. 2.6. The increased corner frequency allows greater increase in impedance from the zero, resulting in the slight increase in magnitude and frequency of the peak. Increased $K_{p}$ values will also generate a resonance peak that directly adds to the magnitude of the 
observed impedance peak.

Increasing the value of $K_{d}$ has a less significant impact on the magnitude of impedance (see Fig. 2.7). From Fig. 2.8, increasing $K_{d}$ reduces the resonance peak and the corner frequencies of the poles of the second term. This reduction in corner frequencies allows for further decrease in the impedance due to the poles before the inertia of the CLS dominates the impedance response. The effects of both the decreased resonance peak and the decreased corner frequencies allows increased $K_{d}$ values to reduce the middle frequency impedance values that the $K_{p}$ gain tends to increase.

To minimize the cost function (2.3), the impedance must be reduced below $\left|Z_{d}\right|$ for over the largest range of impedances possible. Based on the observed effects of $K_{p}$ and $K_{d}$, a combination of the two gains should be employed. The $K_{p}$ value should be increased to decrease low frequency impedance to below $\left|Z_{d}\right|$ and $K_{d}$ must then be increased to compensate for the impedance peak at middle frequencies.

\subsection{Summary}

The current chapter developed design requirements for the response controller of the SOIS. Based on the requirements, directly feeding back the measured deformation $\vec{x}_{d}$ into the position controller was proposed as the SOIS control law. The proposed controller was analysed for a single degree of freedom, linear time invariant system. Conditions on $K_{p}$ and $K_{d}$ for the stability of the control law during interaction were determined. Increasing the $K_{p}$ and $K_{d}$ gains were found to increase performance by both decreasing the perceived impedance and preventing 
the complete compression of the CLS. 


\section{Chapter 3}

\section{Investigation of the Single Degree of Freedom Control Law through Simulation and Experimentation}

To investigate the SOIS controller developed in Chap. 2, the proposed control structure is implemented in simulation and experimentally for a 1DoF robot and CLS. Through simulation, the general behaviours of the controller during interaction and impact are investigated. Simulations also explore the effects of stiction, sensor noise, control saturation and sampling frequency. In simulations, these nonlinearities can be added individually to the ideal linear system, allowing isolation of the effect of each on the ideal controller. The controller is implemented and experiments are performed to investigate the applicability of the control law to real systems with unmodelled nonlinearities, and to compare the proposed SOIS control law to admittance control.

To guide the controller implementation, a general procedure is introduced in 
Sec. 3.1. The procedure requires as inputs the system model and design specifications and outputs the optimal control gains to minimize the cost function (2.3) while meeting all other design requirements and ensuring stability. The hardware of the 1 DoF test bed is then described and a linear model is fit to the observed system dynamics in Sec. 3.2. In Sec. 3.3, a control law is developed for the linear model using the previously described procedure. The results of simulations and experiments are then presented in Sec. 3.4 and Sec. 3.5.1, respectively.

\subsection{Design Procedure}

To take full advantage of the knowledge gained from the analysis of the 1DoF LTI system, a procedure is established to design the control law using a PD position controller given a 1DoF robot and CLS. An integral term is not considered in the current design procedure since the integral term generates non zero velocity of the manipulator in steady state and, in the current safety application, no steady state error is expected which would require the use of an integral term. By introducing a set procedure, the controller can be more easily applied to new scenarios. The design procedure also illustrates the manner in which the results of Chap. 2 and the design specifications interact in implementing the controller.

The currently outlined procedure will seek to design a controller that minimizes the cost function (2.3), while meeting requirements for:

- stability,

- the desired maximum impact velocity before compression $V_{d m a x}$,

- the desired maximum allowable steady state motion of the robot in response 
to initial conditions $\Delta x_{R \max }$,

- and maximum allowable percent overshoot of the position controller when connected to the CLS, PO $\max$.

The procedure determines a range of gains to satisfy these requirements and uses the reduction of impedance to select between the allowable gains. Priority is placed on meeting the requirements of stability, $\Delta x_{R \max }$ and $P O_{\max }$ as failure to meet these conditions results in unexpected motion in the collision response, increasing the likelihood of a second collision. Meeting the requirement of $V_{d \max }$ prevents the complete deformation of the CLS, which would result in a second rigid collision that is dangerous to both the robot and the user. Consequently, satisfying $V_{d \max }$ must be given higher priority over reducing impedance.

The proposed procedure is an extended version of the procedure in [84] that has been adapted for safety. In [84], a design procedure is proposed wherein a system model is proposed, the bounds of stability are determined and a cost function similar to (2.3) is then used to numerically optimize the gains to approach a desired impedance or to minimize impedance. The current procedure extends this method by determining bounds of acceptable gains that not only consider stability but conditions on $V_{d \max }, \Delta x_{R \max }$ and $P O_{\max }$, which are required for safety. The cost function is also changed to (2.3) as safety does not require a specific desired impedance or an arbitrary minimization of impedance; safety requires that the impedance remains below the safe value $\left|Z_{d}\right|$ for as much of the frequency domain as possible. The robot is no longer dangerous below $\left|Z_{d}\right|$ and so further decreases in impedance are not rewarded with current cost function as it is in [84]. 
The current procedure requires as inputs a system model and the design requirements for the given application. The procedure will output acceptable control gains $K_{p}$ and $K_{d}$ for a PD position control law which minimize the cost function (2.3) using the SOIS control methodology. The four step design procedure is summarized in Fig. 3.1 and the four design steps are:

\section{Step 1: Definition of Design Requirements and System Model}

To design the control law, the following must be specified for the system and application:

Estimated robot model parameters, $M_{R}$ and $B_{R}$

Estimated CLS model parameters, $M_{S}, B_{S}$ and $K_{S}$

\section{Maximum CLS deformation $x_{d \max }$}

The maximum CLS deformation $x_{d \max }$ must be specified for the calculation of $V_{d \max }$. In specifying $x_{d \max }$, both the physical constraints of the sensor and the linear CLS model must be considered. The $x_{d \max }$ value must be less than the physical limits of the sensor including the maximum deformation until the CLS becomes rigid or is damaged. The stiffness of the CLS will also vary with the deformation. The maximum CLS deformation must be small enough that the actual stiffness does not differ significantly from the linear approximation $K_{S}$. Should the bounds of the maximum deformation differ in tension and compression, the smaller value should be used. 


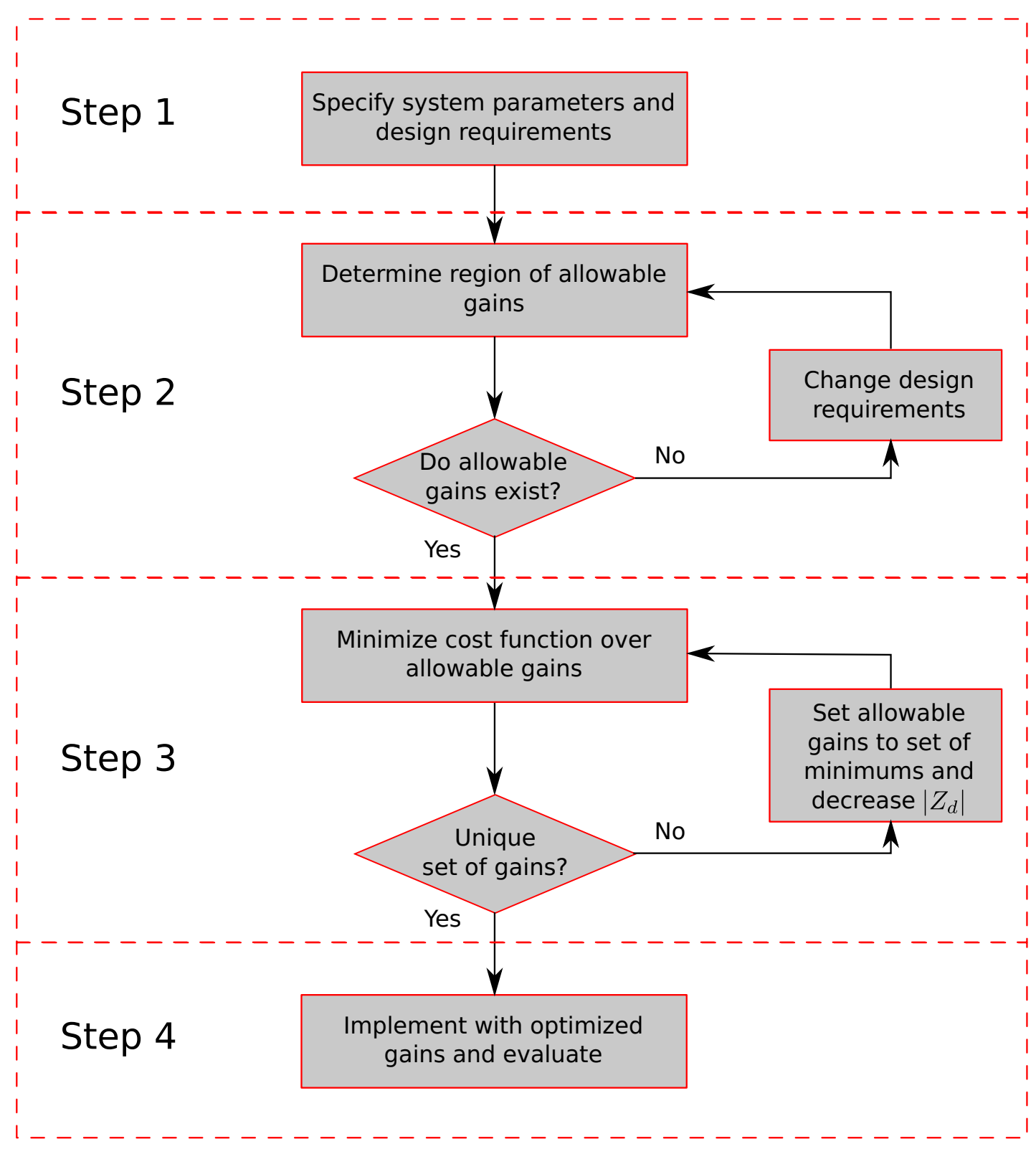

Figure 3.1: Summarizing flow chart of the four step design procedure. 


\section{Maximum actuator output $F_{\text {sat }}$}

The actuator output $F_{\text {act }}$ of a real system has an upper bound $F_{\text {sat }}$. To ensure predictable behaviour for the control law as described in Chap. 2, the commanded output $F_{\text {act }}$ should not exceed $F_{\text {sat }}$ for the operating conditions of the robot.

\section{Desired maximum velocity before complete CLS deformation $V_{d \max }$}

To design a control law, a minimum allowable maximum velocity before complete CLS deformation $V_{d \max }$ must be specified to provide bounds on the acceptable $K_{d}$ and $K_{p}$. In specifying $V_{d m a x}$, care must be taken to ensure the value is attainable. If $V_{d \max }$ is large, the control gains required to ensure $V_{d \max }$ may become too large for the actuator, resulting in saturation. The specified $V_{d \max }$ should be the minimum required for the application and can be determined in two ways. If a maximum safe operating velocity $V_{\max O p}$ and safety factor $F$ are known, $V_{d \max }$ can be determined using (2.23). If the operating range of the robot is not specified, an attainable $V_{d \max }$ can be determined as the maximum velocity the robot can reach when accelerating from rest over a distance of $x_{d \max }$. This method ensures that if the robot starts at $V_{d \max }$, it is able to stop within the distance $x_{d \max }$ without the assistance of the CLS forces or robot friction.

\section{Maximum allowable steady state offset in free motion $\Delta x_{R \max }$}

A maximum allowable steady state motion $\Delta x_{R \max }$ must be defined to determine constraints on $K_{p}$ and $K_{d}$. Small values of $\Delta x_{R \max }$ may overly restrict controller gains; hence, $\Delta x_{R \max }$ should be maximized while considering the application. In determining $\Delta x_{R \max }$, the number of obstacles in the environment and the size 
of the robot must be considered. If many obstacles exist, the probability of a second collision increases and so $\Delta x_{R \max }$ must be kept low. As the size of the robot increases, the specified $\Delta x_{R \max }$ should also increase proportionally so as to prevent overly restricting conditions on the acceptable gains.

\section{Maximum allowable percent overshoot $P O_{\max }$}

The value of $P O_{\max }$ will affect the magnitude of the oscillations perceived in the controller during interaction and the amount of steady state offset in free motion when the manipulator collides with an object and retreats. The value of $P O_{\max }$ should be minimized, with a proposed value of $1 \%$. However, lower values for $P O_{\max }$ can result in restricting controller gains for systems involving large robot inertia $M_{R}$, large CLS stiffness $K_{S}$, and low damping $B_{S}$ and $B_{R}$.

\section{Minimum impedance required to cause injury $\left|Z_{d}\right|$}

The minimization of the cost function,

$$
W=\int_{\Omega_{\min }}^{\Omega_{\max }} C(\Omega) d \Omega
$$

with

$$
C(\Omega)= \begin{cases}\log \left|Z\left(j 10^{\Omega}\right)\right|-\log \left|Z_{d}\right| & \forall\left|Z\left(j 10^{\Omega}\right)\right| \geq\left|Z_{d}\right| \\ 0 & \forall\left|Z\left(j 10^{\Omega}\right)\right|<\left|Z_{d}\right|\end{cases}
$$

requires the specification of $\left|Z_{d}\right|$ in $\mathrm{Ns} \mathrm{m}^{-1}$. If the specified $\left|Z_{d}\right|$ is too large, the optimization may be unable to differentiate between options as all impedances below $\left|Z_{d}\right|$ are considered safe and so have zero cost. In contrast, if $\left|Z_{d}\right|$ is too 
small, the impedance reduction caused by the control law may not be spread uniformly across the frequency domain as one frequency range is decreased significantly while ignoring other frequencies. As a general guideline, it is proposed to use

$$
\left|Z_{d}\right|=\frac{150 \mathrm{~N}}{V_{d \max }} .
$$

Specifying $\left|Z_{d}\right|$ in this way ensures that at the maximum velocity $V_{d \max }$, the robot does not apply more than $150 \mathrm{~N}$, the maximum allowable static force in human-robot interaction, as specified by ISO-10218 [24].

\section{Step 2: Determination of the range of allowable $K_{p}$ and $K_{d}$}

Before minimizing (2.3), the range of allowable $K_{p}$ and $K_{d}$ must be determined using the restrictions of stability, $V_{d \max }, \Delta x_{R \max }$ and $P O_{\max }$, and preventing saturation of the actuator. The conditions that must be satisfied for each restriction are elaborated on below and are based on the derivations in Chap. 2 .

\section{Conditions to ensure stability}

The range of allowable $K_{p}$ and $K_{d}$ that ensure stability of the control law when interacting with a passive environment can be determined conservatively using (2.21) and (2.22). Rearranging both conditions, it is found that for stability $K_{d}$ must be greater than either,

$$
\begin{aligned}
K_{d} & \geq \frac{M_{R} K_{p}}{B_{R}}-B_{R}-B_{S}, \\
K_{d} & \geq \frac{B_{S} K_{p}}{K_{S}} .
\end{aligned}
$$


Both conditions define lines on the $K_{p^{-}} K_{d}$ plane which the selected $K_{d}$ must lie above.

\section{Conditions to achieve $V_{d \max }$}

In examining Fig. 2.4, it is seen that $V_{\max }$ increases with both $K_{p}$ and $K_{d}$. Consequently, the need to achieve $V_{d \max }$ will set a lower bound on the $K_{d}$ for a given $K_{p}$. Equations (2.25)-(2.29) could be employed with the system model and $x_{d \max }$ to determine numerically the minimum $K_{d}$ required for a given $K_{p}$ to satisfy $V_{d \max }$.

\section{Conditions to achieve $\Delta x_{R \max }$}

To ensure $\Delta x_{R}$ is below the desired $\Delta x_{R \max }$, one must employ (2.15) with the maximum expected initial conditions. From (2.15), it is noted that the control gains can only influence the portions $\Delta x_{R}$ due to initial CLS mass velocity $\dot{x}_{S}(0)$ and CLS deformation $x_{d}(0)$; the steady state offset due to the initial robot velocity $\dot{x}_{R}(0)$ is influenced only by the robot properties. For this reason, only $\dot{x}_{S}(0)$ and $x_{d}(0)$ are considered in the control design. To prevent maximum steady state offsets in free motion from exceeding $\Delta x_{R \max }$, the $\Delta x_{R}$ due to the largest expected conditions must be analysed. For the application, the maximum possible $x_{d}(0)$ is specified as $x_{d \max }$. Specifying a maximum $\dot{x}_{S}(0)$ is difficult as the environment is unknown and collisions are unexpected; however, a possible estimate would be $V_{d \max }$ as it does define the maximum velocity the control law is expected to tolerate. No relationship exists between $x_{d}(0)$ and $\dot{x}_{S}(0)$, but it can be assumed that if the controller is behaving appropriately, the maximum CLS deformation and the maximum allowable impact velocity $V_{d \max }$ should not occur simultaneously. To prevent overly restricting conditions, it is assumed that if $x_{d}(0)$ is at 
the maximum value, $\dot{x}_{S}(0)$ is zero and vice versa. For simplicity, between these two conditions, the $x_{d}(0)$ and $\dot{x}_{S}(0)$ are assumed to vary linearly such that,

$$
\frac{\dot{x}_{S}(0)}{V_{d \max }}+\frac{x_{d}(0)}{x_{d \max }}=1 .
$$

Under these conditions, (2.15) can be employed to find the following two conditions to satisfy the requirements of $\Delta x_{R \max }$,

$$
\begin{aligned}
& K_{p} \leq K_{S}\left(\frac{B_{R}}{M_{S}} \frac{\Delta x_{R \max }}{V_{d \max }}-1\right) \\
& \frac{B_{S} K_{p}}{K_{S}}-B_{R} \frac{\Delta x_{R \max }}{x_{d \max }} \leq K_{d} \leq \frac{B_{S} K_{p}}{K_{S}}+B_{R} \frac{\Delta x_{R \max }}{x_{d \max }} .
\end{aligned}
$$

Conditions (3.5) and (3.6) represent a closing of the region of acceptable control gains in the $K_{p}-K_{d}$ plane, as (3.5) provides an upper bound on $K_{p}$ values and (3.6) provides upper and lower bounds on $K_{d}$ for a given $K_{p}$. The restriction (3.5) may not be able to be satisfied for a given combination of $\Delta x_{R \max }$ and $V_{d \max }$. In such cases, $\Delta x_{R \max }$ will need to be increased or $V_{d \max }$ must be decreased.

\section{Conditions to remain below $P O_{\max }$}

Before determining conditions to remain below $P O_{\max }, P O_{\max }$ is first converted into an equivalent damping ratio $\zeta_{\min }$ based on the second order method described in [91]. Once the $\zeta_{\min }$ is determined, the condition required to satisfy this minimum value for the combined CLS and manipulator assuming a fixed CLS mass is

$$
K_{d} \geq 2 \zeta_{\min } \sqrt{M_{R}\left(K_{S}+K_{p}\right)}-B_{R}-B_{S} .
$$


The above equation sets another minimum condition on the required value of $K_{d}$ that increases with $K_{p}$.

\section{Conditions to prevent saturation}

To prevent saturation of the control output, one must ensure that the commanded output is less than $F_{\text {sat }}$ for all possible position and velocity errors. The maximum position and velocity errors expected in normal operation are $x_{d \max }$ and $V_{d \max }$, respectively. The maximum possible position and velocity errors are assumed to not occur simultaneously and, for simplicity, are assumed to vary linearly in the same manner described for $x_{d}(0)$ and $\dot{x}_{S}(0)$. Under these conditions, saturation can be prevented by satisfying both,

$$
\begin{aligned}
K_{p} & \leq \frac{F_{\text {sat }}}{x_{d \max }} \\
K_{d} & \leq \frac{F_{\text {sat }}}{V_{d \max }}
\end{aligned}
$$

The above conditions must be combined to determine the range of allowable $K_{p}$ and $K_{d}$ gains. If such gains do not exist, $\Delta x_{R \max }, P O_{\max }$ and $V_{d \max }$ must be adjusted to relax the $K_{p}$ and $K_{d}$ restrictions, as these are the only values not determined directly by the system. In varying the parameters, the most prohibitive restrictions must be determined and Table 3.1 should be consulted to determine the parameters to relax the restrictions. From Table 3.1, it is noted that the restriction on $K_{p}$ due to saturation and the stability conditions are not affected by changes in $\Delta x_{R \max }, P O_{\max }$ and $V_{d \max }$. These restrictions are due to the system employed and so the system must be changed to adjust these 
Table 3.1: The effect of varying $\Delta x_{R \max }, P O_{\max }$ and $V_{d \max }$ on the required controller conditions. - indicates no effect, $+\propto$ indicates the condition is relaxed by increasing the value and $-\propto$ indicates the condition is relaxed by decreasing the value.

\begin{tabular}{|l|l|l|l|l|}
\hline Requirement & Equation & $\Delta x_{R \max }$ & $P O_{\max }$ & $V_{d \max }$ \\
\hline \multirow{2}{*}{ Stability } & $(3.2)$ & - & - & - \\
\cline { 2 - 5 } & $(3.3)$ & - & - & - \\
\hline \multirow{2}{*}{$V_{d \max }$} & - & - & - & $-\propto$ \\
\hline \multirow{2}{*}{$\Delta x_{R \max }$} & $(3.5)$ & $+\propto$ & - & $-\propto$ \\
\cline { 2 - 5 } & $(3.6)$ & $+\propto$ & - & - \\
\hline$P O_{\max }$ & $(3.7)$ & - & $+\propto$ & - \\
\hline \multirow{2}{*}{ Actuator Saturation } & $(3.8)$ & - & - & - \\
\cline { 2 - 5 } & $(3.9)$ & - & - & $-\propto$ \\
\hline
\end{tabular}

conditions.

\section{Step 3: Minimizing impedance over the region of allowable} gains

To select the final controller from the viable alternatives, it is proposed to minimize the cost function (2.3) over the acceptable range of $K_{p}$ and $K_{d}$ gains. The exact method of minimization is arbitrary and methods from optimal control could be employed to analytically minimize (2.3). However, as the set of allowable gains is bounded and only two values $\left(K_{p}\right.$ and $\left.K_{d}\right)$ must be determined, an exhaustive search is also appropriate, as in [84]. An exhaustive search is simple and eliminates the need to minimize the nonlinear, piecewise cost function (2.3) analytically; consequently, it is employed in the current work. 
In optimizing (2.3), multiple control gains may produce similar costs. This may occur if $\left|Z_{d}\right|$ is set too high and multiple control gains may reduce the impedance below $\left|Z_{d}\right|$ for the majority of the frequency range. In such cases, the cost function (2.3) with a reduced $\left|Z_{d}\right|$ value should be minimized again over the set of control gains that previously minimized the cost function with the higher $\left|Z_{d}\right|$ value. This process is repeated as necessary until a single value remains.

\section{Step 4: Implement, test and adjust control law}

After the final control gains have been selected, the controller should be implemented on the hardware and the performance tested with controlled impact and interaction tests. The hardware implementation includes nonlinear effects such as dry friction and backlash not captured with linear modelling. These effects may result in undesired behaviour such as instability and limit cycles that will need to be addressed by adjustments to the control law.

\subsection{Hardware}

To experimentally validate the proposed control law and to provide a realistic model for simulations, a 1DoF test platform and CLS are developed and characterized. 


\subsubsection{DoF test platform}

\subsubsection{Design}

The test platform can be seen in Fig. 3.2. The test platform consists of a Maxon EC Powermax 200 DC brushless motor connected to an arm through a 1:565 reduction two stage gear box. The second stage of the gear box has low backlash, allowing for accurate arm positioning. The motor shaft is equipped with a 500

pulse per revolution encoder (Avago hedl-5540 a11) allowing a resolution of $\frac{1}{3139}^{\circ}$. The motor is controlled using a Maxon DES70/10 servoamplifier in current control mode. A National Instruments PCI 6229 DAC card is used to interface the control PC with the hardware. The control law and data collection are implemented at a $2 k H z$ sampling frequency using a $\mathrm{xPC}$ target programmed using the Simulink Real-Time toolbox in Matlab.

A mounting stand was placed along the path of the arm. During impact experiments, a Transducer Techniques MLP 75 force sensor was mounted to measure forces between the arm and the stand. During interaction experiments, the CLS could be connected to the mounting stand through springs to test stability with interaction.

To ensure safety, the host PC was capable of disabling the servoamplifier through a digital output should large forces or displacements be measured. An emergency stop was also placed near the operator should the software stop fail. Additionally, the maximum servoamplifier continuous current was set below the maximum operating range of the motor to limit forces. The mounting stand was also designed to yield before failure through a breakaway connection. 


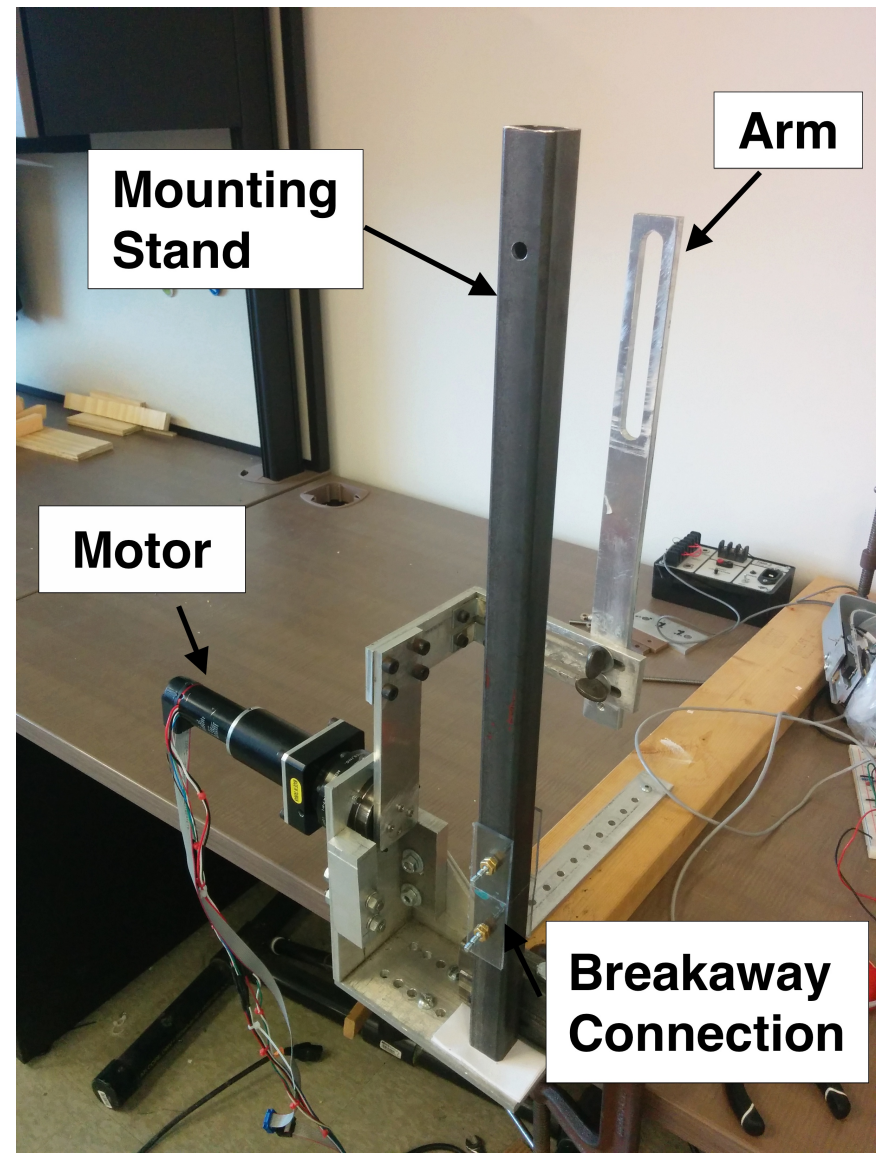

Figure 3.2: Labelled photograph of the 1DoF test platform 


\subsubsection{Linear Model of the Test Plaform}

The proposed linear mass-damper model of the system is calibrated to find parameters $M_{R}$ and $B_{R}$, see Appendix A.2 for details. The reflected inertia of the motor and gearbox was calculated as $1.363 \mathrm{~kg} \mathrm{~m}^{2}$ using the values provided by the manufacturer. The damping coefficient was determined experimentally to be $5.1 \mathrm{Nms}$.

\subsubsection{DoF Compliant Limb Sensor}

\subsubsection{Design}

The current design of the 1DoF CLS (seen in Fig. 3.3) employs the use of two aluminium brackets connected by a linear slider as the external shell and base. The CLS compliance is introduced through a compression and a tension spring between the two brackets. The current springs were chosen with a low stiffness to allow the motor to deform the CLS without assistance, facilitating experiments. A PTE60-151B-102B2 potentiometer measured the displacement between the two brackets. The potentiometer was calibrated using calipers and voltage measurements from the 1DoF system DAC. A first order analogue low pass filter with a high corner frequency $(1427 \mathrm{~Hz})$ was included to eliminate motor noise.

\subsubsection{Force Estimation}

The force-displacement curve of the CLS was estimated by hanging known masses from the external shell. The resulting force-displacement curve for the current set up can be seen in Fig. 3.4a, where tension is positive and compression is 


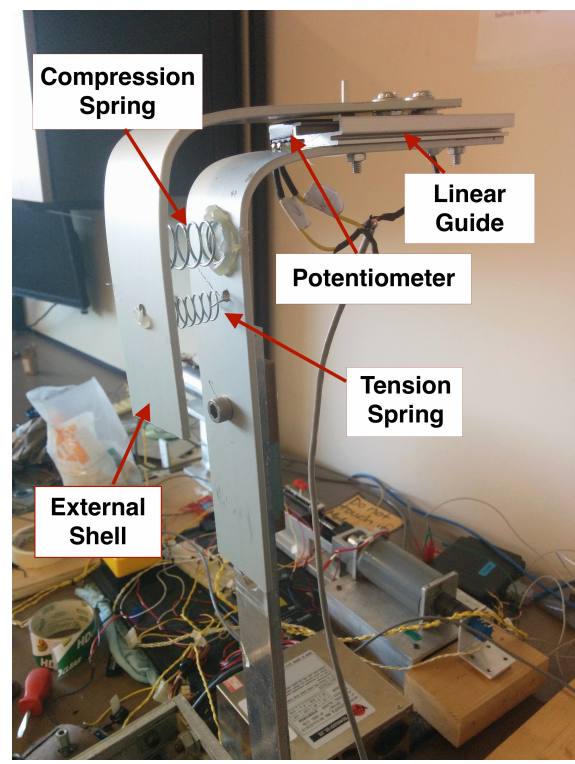

(a) 1DoF prototype

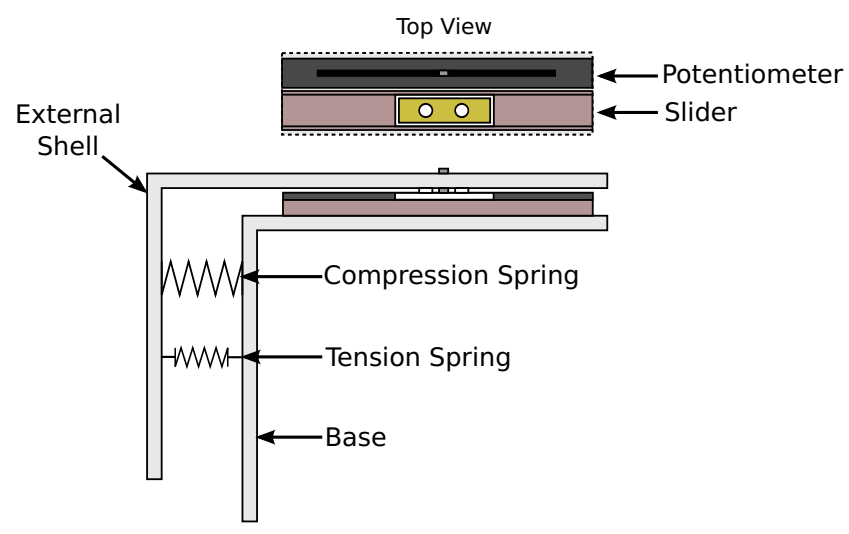

(b) Schematic

Figure 3.3: 1DoF compliant limb sensor

negative. The nonlinear tension relationship was due to losing contact between the compression spring and outer shell. The disconnection occurred at approximately $8 \mathrm{~mm}$ and a change in stiffness was expected at this point. In the region close to the origin $( \pm 1.8 \mathrm{~mm})$, the dry friction exceeded the sum of the applied weight and spring force so that the sensor would not move, resulting in the horizontal force-displacement relationship.

Force estimates using the CLS displacement will be inaccurate due to the dry friction. Despite the inherent uncertainty, an approximate relationship between position and deformation can be found by accounting for friction by shifting both the compression and tension data to align the two curves, see Fig. 3.4b. A linear regression can then approximate the force-displacement relationship for displacements up to $8 \mathrm{~mm}$. For deformations above $8 \mathrm{~mm}$, a second linear fit was 


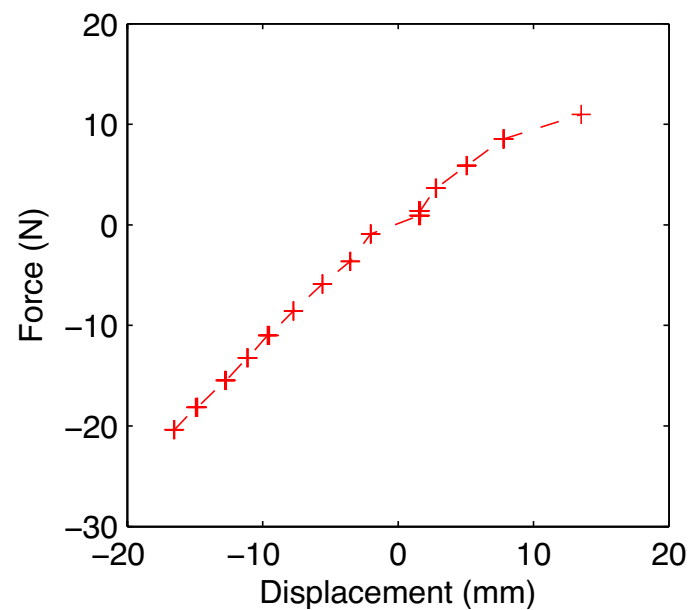

(a) Observed force displacement data for the 1DoF CLS

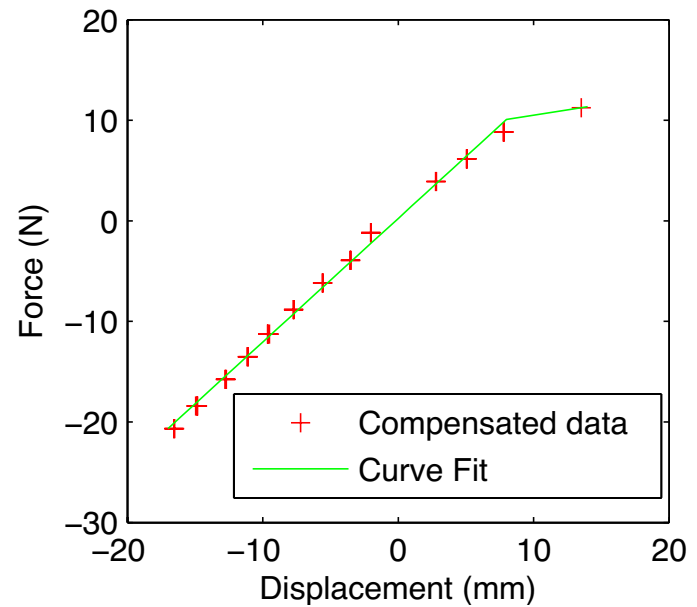

(b) Curve fit of force-displacement behaviour of the CLS with compensation for dry friction

Figure 3.4: Force-displacement characteristics for 1DoF CLS

used to account for the disconnection of the compression spring. The resulting equations are,

$$
f(d)= \begin{cases}1.2294 \mathrm{~N} \mathrm{~mm}^{-1} d-0.2434 \mathrm{~N} & : d<8 \mathrm{~mm} \\ 0.2144 \mathrm{~N} \mathrm{~mm}^{-1} d+8.3630 \mathrm{~N} & : d>8 \mathrm{~mm}\end{cases}
$$

\subsubsection{Sensor Model}

The CLS is modelled as a mass-spring-damper to allow controller design and system simulations. From the external shell dimensions, the mass of the shell was estimated as $0.098 \mathrm{~kg}$ and the stiffness was previously determined to be $1229.4 \mathrm{~N} \mathrm{~m}^{-1}$. The damping coefficient for the model was estimated based on experimental results as $20.8 \mathrm{~N} \mathrm{~s} \mathrm{~m}^{-1}$, see Appendix A.3 for details. The nonlinear effect of dry friction is neglected in the current model; however, the model 
is suitable to provide an initial estimate of control parameters and a system for simulations.

\subsection{Control Design}

With the 1DoF test platform and CLS developed and characterized, steps 1-3 of the design procedure described in Sec. 3.1 are performed to select the $K_{p}$ and $K_{d}$ gains.

\section{Step 1: Definition of Design Requirements and System}

\section{Model}

The required design restrictions and parameters are specified in Table 3.2. The values for $M_{R}$ and $B_{R}$ are the inertia and damping of the motor reflected to the location of the CLS using the radius of the arm $(0.375 \mathrm{~m})$. The maximum deformation $x_{d \max }$ is selected to be $8 \mathrm{~mm}$ to avoid the change in stiffness in the tension behaviour of the spring (see Fig. 3.4b). The minimum dangerous impedance $\left|Z_{d}\right|$ is specified using (3.1).

The values for $\Delta x_{R \max }$ and $P O_{\max }$ in Table 3.2 were chosen after initially setting requirements of $0.01 \mathrm{~m}$ and $1 \%$, respectively. These values of $\Delta x_{R \max }$ and $P O_{\max }$ did not result in allowable gains and so were adjusted. The $P O_{\max }$ was increased more than the $\Delta x_{R \max }$ due to the high inertia and low damping nature of the investigated system. In addition, increases in $P O_{\max }$ create larger oscillations; however, the stability of the oscillations is guaranteed. A maximum $10 \%$ overshoot indicates that, at worst, the controller will generate a tenth of the 
original deformation and force in the opposite direction when interacting with the environment. As the forces are small for the current set up, a tenth of the value will not pose a safety concern.

Table 3.2: Design specifications for the $1 \mathrm{DoF}$ test platform and CLS to be used in controller design

\begin{tabular}{|l|l|l|}
\hline \multirow{4}{*}{ Robot Parameters } & $M_{R}$ & $9.69 \mathrm{~kg}$ \\
\cline { 2 - 3 } & $B_{R}$ & $36.27 \mathrm{~N} \mathrm{~s} \mathrm{~m}^{-1}$ \\
\cline { 2 - 3 } & $F_{\text {sat }}$ & $166.34 \mathrm{~N}$ \\
\hline \multirow{4}{*}{ CLS Parameters } & $M_{S}$ & $0.098 \mathrm{~kg}$ \\
\cline { 2 - 3 } & $B_{S}$ & $20.80 \mathrm{~N} \mathrm{~s} \mathrm{~m}^{-1}$ \\
\cline { 2 - 3 } & $K_{S}$ & $1229.4 \mathrm{~N} \mathrm{~m}^{-1}$ \\
\cline { 2 - 3 } Design Restrictions & $x_{\text {dmax }}$ & $0.008 \mathrm{~m}$ \\
\hline & $V_{\text {dmax }}$ & $0.3 \mathrm{~m} \mathrm{~s}^{-1}$ \\
\cline { 2 - 3 } & $\Delta x_{R \max }$ & $0.04 \mathrm{~m}$ \\
\cline { 2 - 3 } & $\left|Z_{d}\right|$ & $500 \mathrm{~N} \mathrm{~s} \mathrm{~m}^{-1}$ \\
\cline { 2 - 3 } & $P O_{\max }$ & $10 \%$ \\
\hline
\end{tabular}

\section{Step 2: Determination of the range of allowable $K_{p}$ and $K_{d}$}

The conditions on $K_{p}$ and $K_{d}$ to meet the requirements of stability, $\Delta x_{R \max }$ and $P O_{\text {max }}$, and to prevent saturation of the actuator for the current system are: 


\section{To ensure stability:}

$$
\begin{aligned}
K_{d} & \geq 0.267 \mathrm{~s}_{p}-57.07 \mathrm{Ns} \mathrm{m}^{-1}, \\
& \text { Or } \\
K_{d} & \geq 0.0169 \mathrm{~s}_{p} .
\end{aligned}
$$

To ensure $\Delta x_{R \max }$ :

$$
\begin{aligned}
& K_{p} \leq 120104 \mathrm{~N} \mathrm{~m}^{-1} \\
& 0.0169 \mathrm{~s}_{p}-181.35 \mathrm{~N} \mathrm{~s} \mathrm{~m}^{-1} \leq K_{d} \leq 0.0169 \mathrm{~s}_{p}+181.35 \mathrm{~N} \mathrm{~s} \mathrm{~m}^{-1}
\end{aligned}
$$

To ensure $P O_{\max }$ :

$$
K_{d} \geq 1.2 \sqrt{9.69 \mathrm{~kg}\left(K_{p}+1229.4 \mathrm{~N} \mathrm{~m}^{-1}\right)}-57.07 \mathrm{~N} \mathrm{~s} \mathrm{~m}^{-1}
$$

\section{To prevent saturation:}

$$
\begin{aligned}
& K_{p} \leq 20792 \mathrm{~N} \mathrm{~m}^{-1} \\
& K_{d} \leq 554.47 \mathrm{~N} \mathrm{~s} \mathrm{~m}^{-1}
\end{aligned}
$$

The lower bounds on $K_{d}$ to ensure $V_{d \max }$ were determined numerically using (2.25)-(2.29), and can be seen in Fig. 3.5b. For the current system, the restrictions $K_{p}$ and $K_{d}$ gains and the region of allowable gains are illustrated in Fig. 3.5 and Fig. 3.6, respectively. From Fig. 3.6, the range of allowable gains is bounded for the current implementation by the restrictions of $P O_{\max }, F_{\text {sat }}, \Delta x_{R \max }$ and 


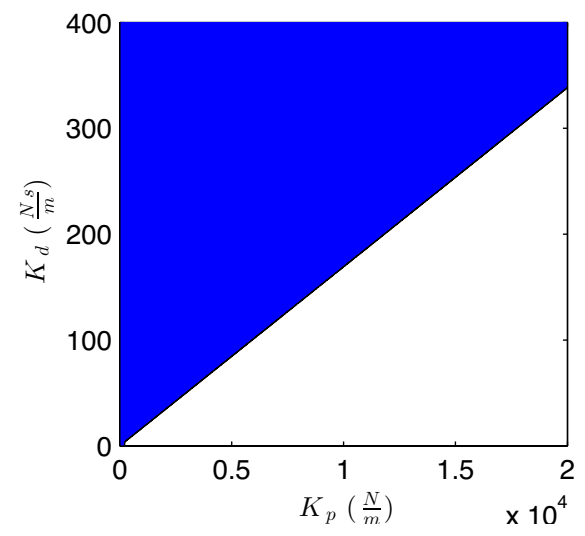

(a) Stability

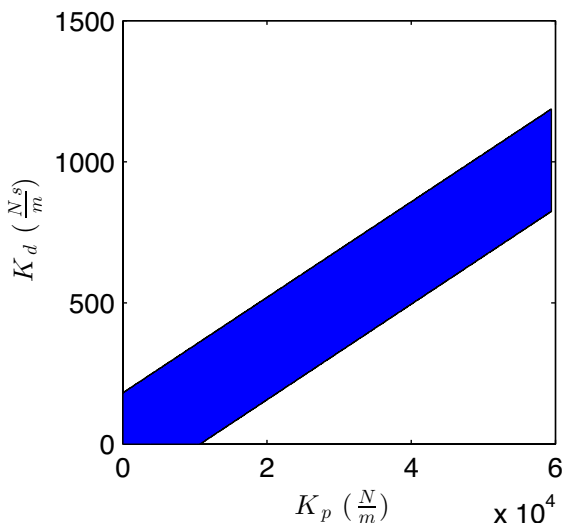

(c) $\Delta x_{R \max }$

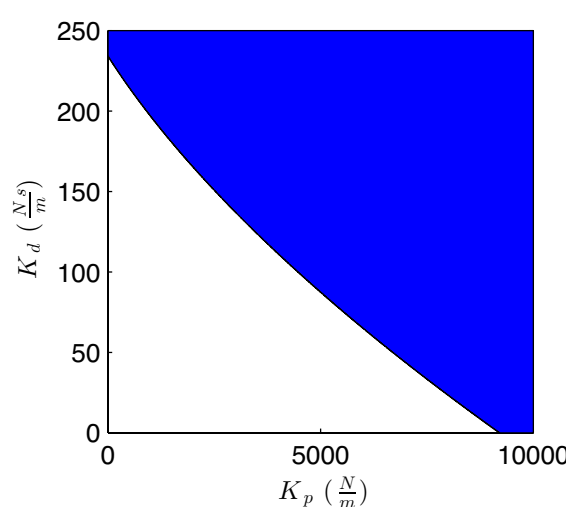

(b) $V_{d \max }$

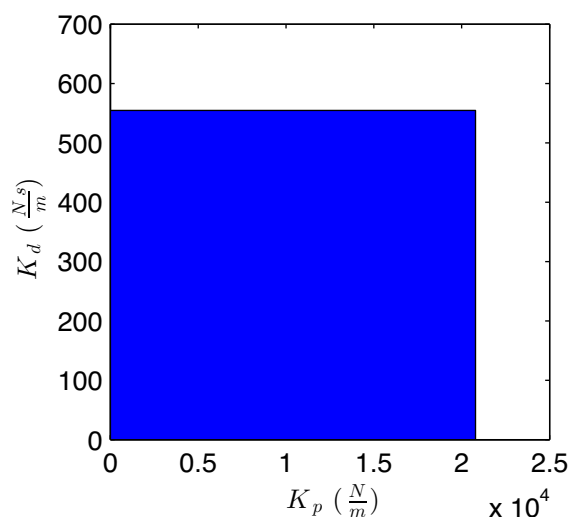

(d) $F_{\text {sat }}$

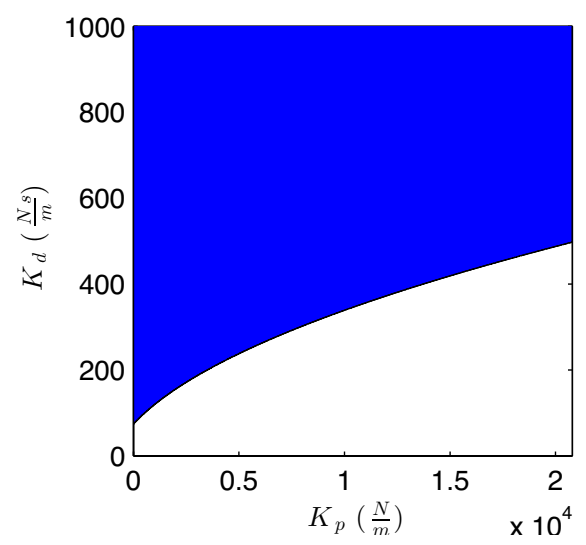

(e) $P O_{\max }$

Figure 3.5: The range of allowable $K_{d}$ and $K_{p}$ values for each restriction with blue indicating the allowable values. 


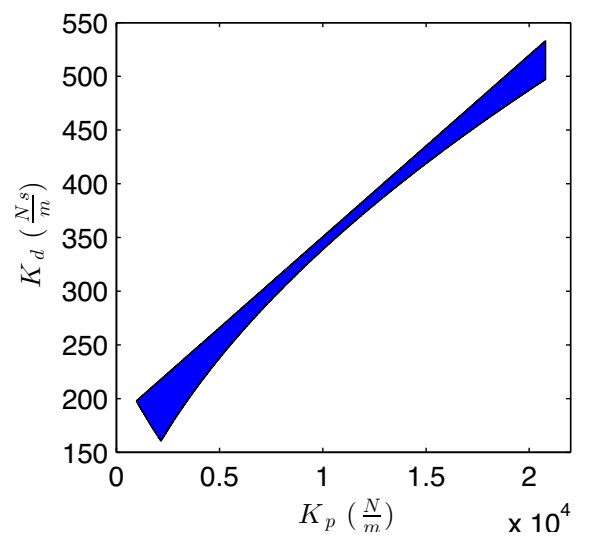

Figure 3.6: Range of allowable $K_{p}$ and $K_{d}$ gains incorporating all restrictions with blue indicating allowable gains.

$V_{d \max }$

\section{Step 3: Minimizing impedance over the region of allowable gains}

To minimize the impedance over the region of allowable gains, an exhaustive search was performed. The original $\left|Z_{d}\right|$ value was too large to differentiate between combinations of gains as was the $\left|Z_{d}\right|$ value determined using the pain threshold of $50 \mathrm{~N}$ [26]. This failure to differentiate was due to the low maximum velocity and low friction and stiffness found in the CLS and robot. The final selected $\left|Z_{d}\right|$ value was $3.33 \mathrm{~N} \mathrm{~s} \mathrm{~m}^{-1}$ and the results of the exhaustive search can be seen in Fig. 3.7. In the current instance, the cost decreases with increased gains. This is expected from the trends found in Chap. 2; however, this is not guaranteed in all applications due to the potential to cause a resonance peak with increased $K_{p}$ values. Based on the optimization, the currently selected gains are $K_{p}=$ $20792 \mathrm{~N} \mathrm{~m}^{-1}$ and $K_{d}=533.1 \mathrm{~N} \mathrm{~s} \mathrm{~m}^{-1}$. For the selected gains, the bode diagram 


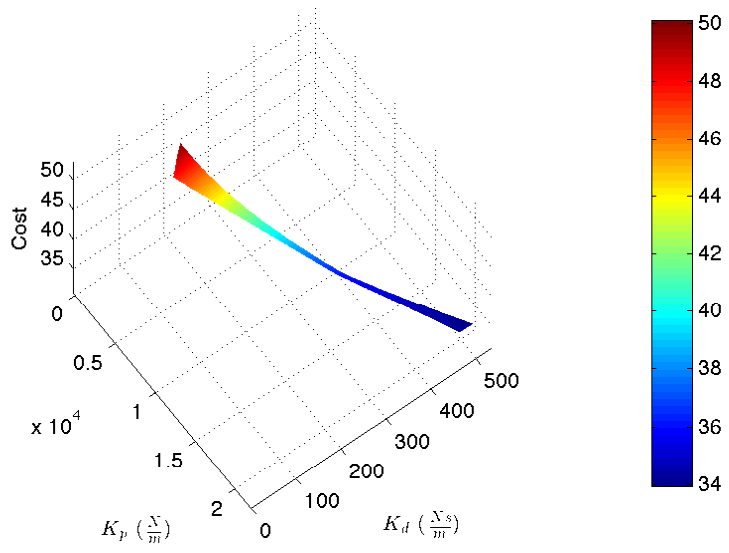

Figure 3.7: Results of the exhaustive search to minimize the cost function.

of the impedance can be seen in Fig. 3.8. From Fig. 3.8, the impedance is decreased over the majority of the frequency range with large decreases occurring at low frequencies due to the large $K_{p}$ gain. One exception to the observed decrease in impedance is the slight increase near $100 \mathrm{rad} \mathrm{s}^{-1}$. This increase is caused by the formation of a resonance peak due to the large $K_{p}$ value with respect to the $K_{d}$ value. As the percent overshoot was allowed to reach up to $10 \%$, the resonance peak is expected due to the underdamped nature of the position control law.

\subsection{Simulation}

Simulations are performed to investigate the behaviour of the previously designed control law. Nonlinearities, including stiction and force saturation, are also simulated to observe their effect on the controller. To allow simulation of these nonlinearities, the current simulations are performed in the time domain. 

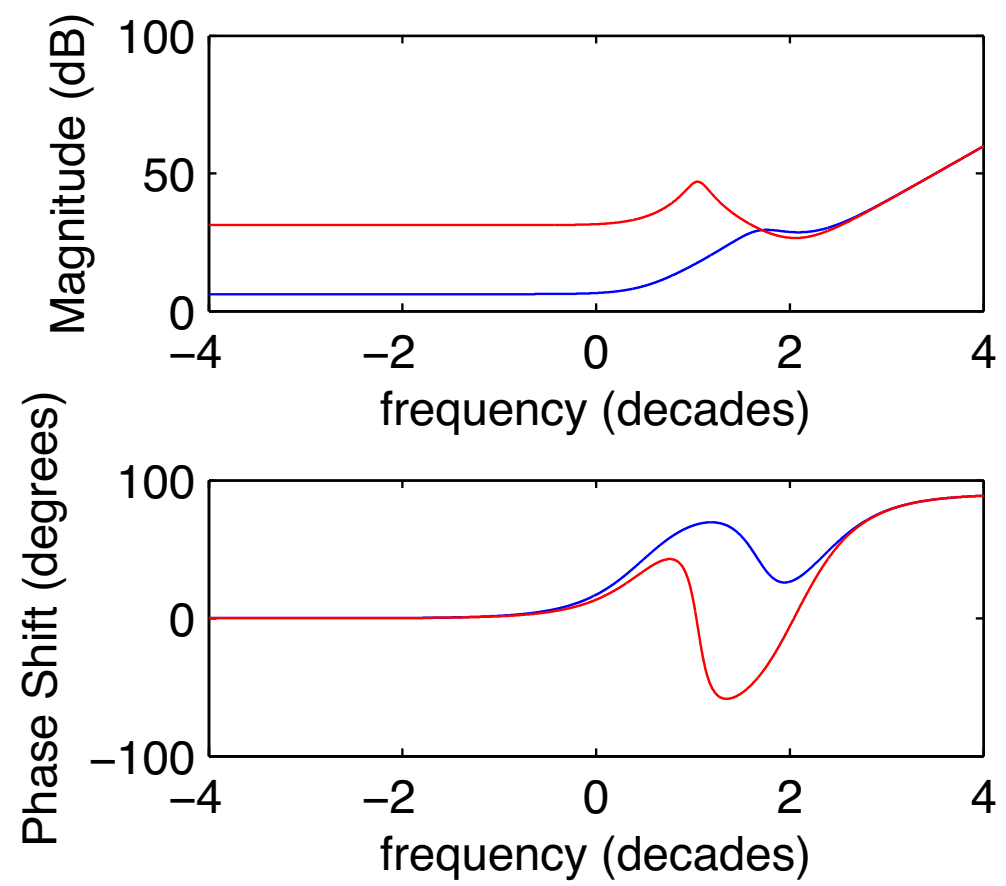

Figure 3.8: Bode diagram of the controlled (blue) and original (red) impedance of the $1 \mathrm{DoF}$ robot. 


\subsubsection{System Model}

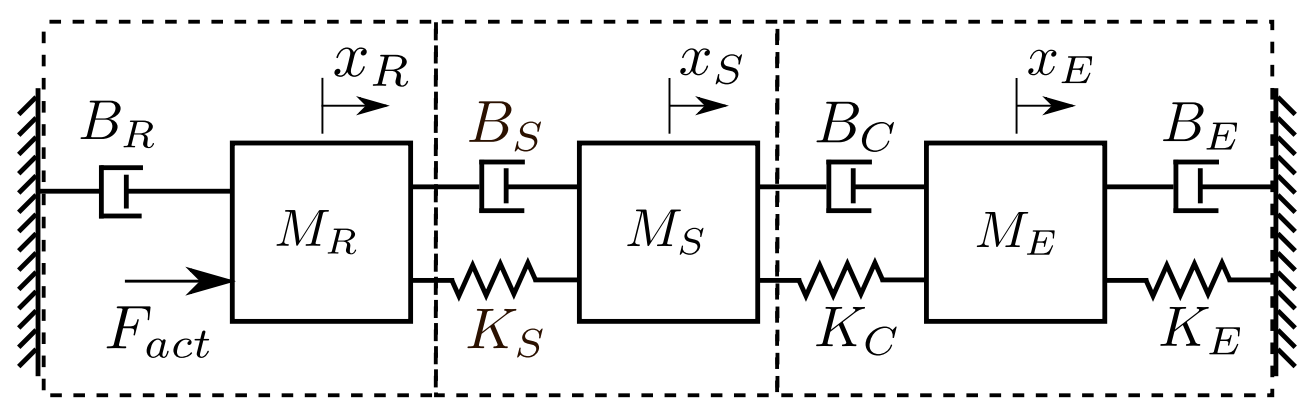

\section{Robot Model CLS Model Environment} Model

Figure 3.9: Linear model employed for current simulations.

To simulate the linear 1DoF control law, the model shown in Fig. 3.9 is proposed, where $K_{C}$ and $B_{C}$ are the contact stiffness and damping, and $M_{E}, K_{E}$ and $B_{E}$ are the environment inertia, stiffness and damping, respectively. The equations of motion for the system are,

$$
\overrightarrow{\dot{x}}=A \vec{x}+B F_{\text {act }}
$$


where

$$
\begin{aligned}
& \vec{x}=\left[x_{R}, x_{S}, x_{E}, \dot{x}_{R}, \dot{x}_{S}, \dot{x}_{E}\right]^{T}
\end{aligned}
$$

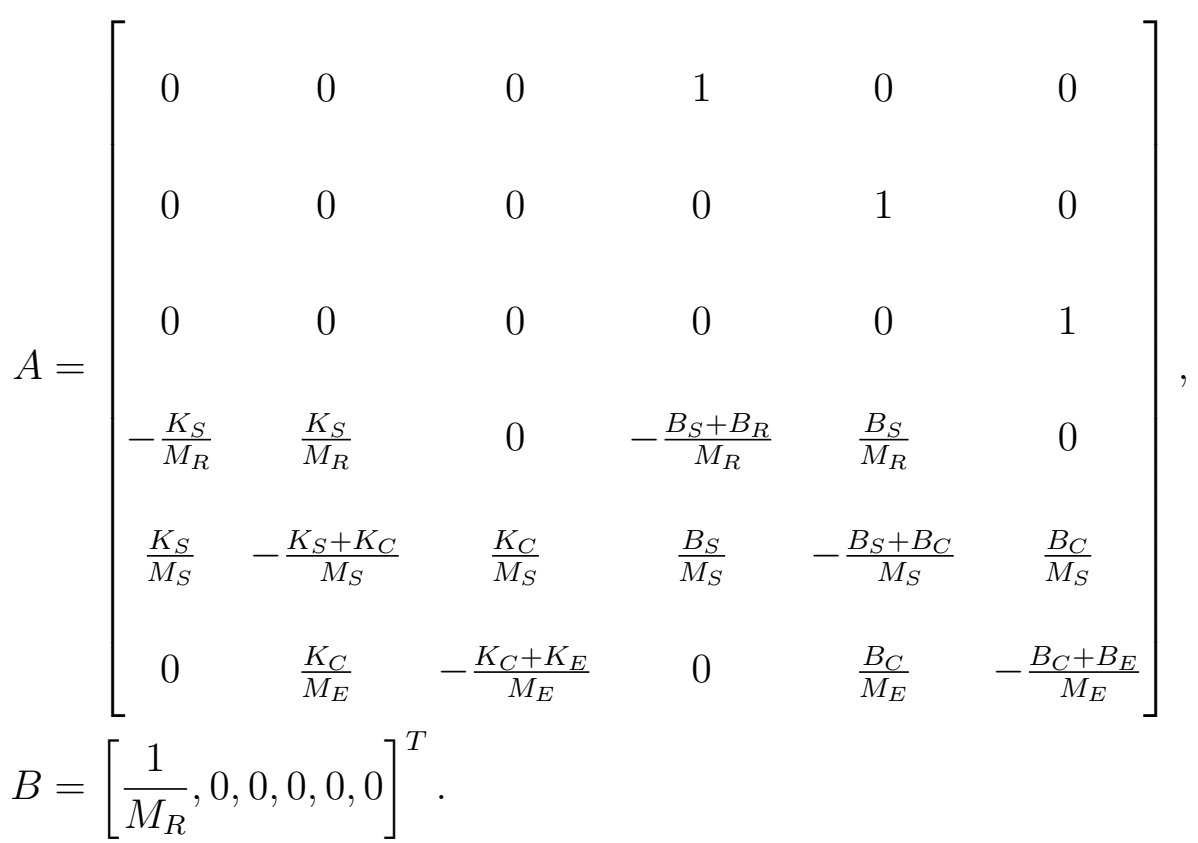

For the impact simulations presented below, the environment model is replaced with a head model consisting of a free mass interacting with the CLS model through the contact model presented in [30]. The parameters employed can be found in Appendix A.4.

For each condition presented, the system will be simulated while interacting with varying environments given an initial CLS deformation of $4 \mathrm{~mm}$. Impact simulations were also performed with both a rigid environment that the CLS is assumed to be connected to and the head model, with impact velocities of $0.15 \mathrm{~m} \mathrm{~s}^{-1}, 0.3 \mathrm{~ms}^{-1}$, and $0.6 \mathrm{~ms}^{-1}$. The environment parameters with which the system interacts can be found in Table 3.3; the parameters were selected to illustrate interaction with inertias, springs and lightly damped combinations of 
Table 3.3: Parameters for various simulated environments.

\begin{tabular}{|l|l|l|l|l|l|}
\hline & $K_{C}$ & $B_{C}$ & $M_{E}$ & $K_{E}$ & $B_{E}$ \\
\hline Environment 1 & $100 \mathrm{~N} \mathrm{~m}^{-1}$ & 0 & $0.01 \mathrm{~kg}$ & $100 \mathrm{~N} \mathrm{~m}^{-1}$ & 0 \\
\hline Environment 2 & $10 \mathrm{kN} \mathrm{m}^{-1}$ & 0 & $0.01 \mathrm{~kg}$ & $10 \mathrm{kN} \mathrm{m}^{-1}$ & 0 \\
\hline Environment 3 & $1 \mathrm{MN} \mathrm{m}^{-1}$ & 0 & $100 \mathrm{~kg}$ & 0 & 0 \\
\hline Environment 4 & $1 \mathrm{MN} \mathrm{m}^{-1}$ & 0 & $0.01 \mathrm{~kg}$ & 0 & 0 \\
\hline Environment 5 & $1 \mathrm{kN} \mathrm{m}^{-1}$ & $10 \mathrm{~N} \mathrm{~s}^{-1}$ & $1 \mathrm{~kg}$ & $1 \mathrm{kN} \mathrm{m}^{-1}$ & $10 \mathrm{~N} \mathrm{~s} \mathrm{~m}^{-1}$ \\
\hline Environment 6 & $10 \mathrm{kN} \mathrm{m}^{-1}$ & $100 \mathrm{~N} \mathrm{~s} \mathrm{~m}^{-1}$ & $1 \mathrm{~kg}$ & $10 \mathrm{kN} \mathrm{m}^{-1}$ & $100 \mathrm{~N} \mathrm{~s} \mathrm{~m}^{-1}$ \\
\hline Rigid Wall & $1 \mathrm{MN} \mathrm{m}^{-1}$ & $10 \mathrm{kN} \mathrm{m}^{-1}$ & $10000 \mathrm{~kg}$ & $1 \mathrm{MN} \mathrm{m}^{-1}$ & $10 \mathrm{kN} \mathrm{s} \mathrm{m}^{-1}$ \\
\hline
\end{tabular}

the two. With the exception of simulations involving an impact with a rigid wall, all simulations were performed using the ode45 function in Matlab. A subset of the results is presented in the sections below, further graphs can be found in Appendix A.5.

\subsubsection{DoF LTI system}

The 1DoF LTI system is simulated with and without the controller to view the general behaviour of the control law. The observed behaviour will serve as a baseline for comparison when subsequent unmodelled dynamics are added.

From Fig. 3.10, it is seen that the proposed control law rapidly reduces the measured CLS deformation in comparison to the uncontrolled system dynamics. By reducing the measured deformation, the controlled robot follows the motion of the connected environment, resulting in large, rapid motions for some cases (see Fig. 3.11a). Due to the system attempting to follow the CLS mass position, the exact shape of the controller response is not fixed, but depends on the dynamics 
of the environment the robot is interacting with. However, it is noted that most responses include overshoot in the CLS deformation due to the underdamped nature of the controller.

By reducing the measured deformation, the controller ensures the CLS is not completely compressed when colliding with a rigid environment, even at double the required $V_{d \max }$ (see Fig. 3.12c). Without the control law, the robot simply collides with the environment and deforms the CLS, resulting in deformations significantly larger than the desired maximum of $8 \mathrm{~mm}$. Regarding contact forces, Fig. 3.13 indicates that the control law is unable to minimize the initial peak impact forces. These forces are due to the inertia of the CLS and so the inability of the controller to affect these peaks stems from controller's inability to affect perceived CLS inertia. These forces increase with greater impact velocity. However, the control law does prevent the subsequent increase in contact forces due to CLS deformation, see Fig. 3.13.

\subsubsection{DoF linear system with force saturation}

In Fig. 3.14, the effect of saturating the actuator force is seen during interaction and impact. Saturation of the actuator force does not significantly affect the behaviour of the controller when pressed against an environment, due to the low forces required of the robot to follow the motion of the external CLS shell (see Fig. 3.14a). However, saturation of the force significantly affects the response of the controller to impacts due to the high initial velocity causing saturation. 


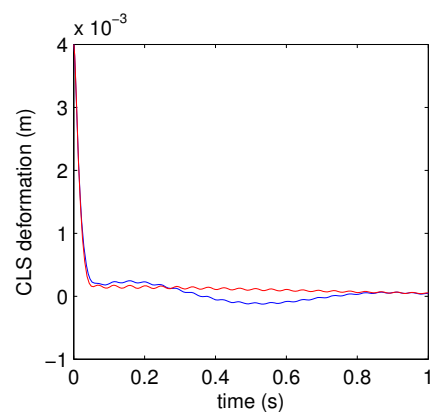

(a) Environment 1

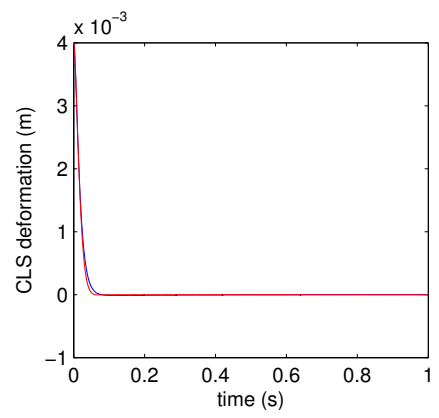

(d) Environment 4

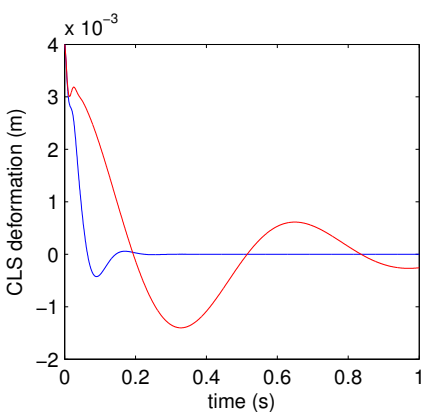

(b) Environment 2

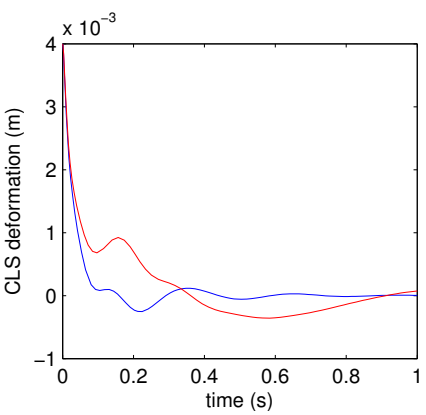

(e) Environment 5

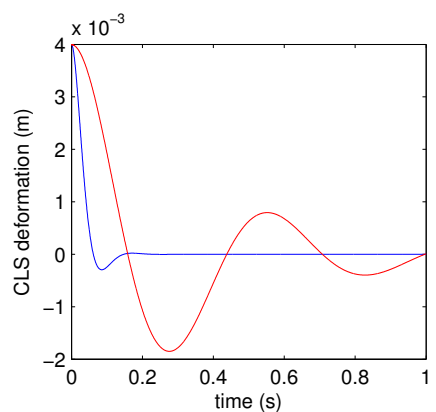

(c) Environment 3

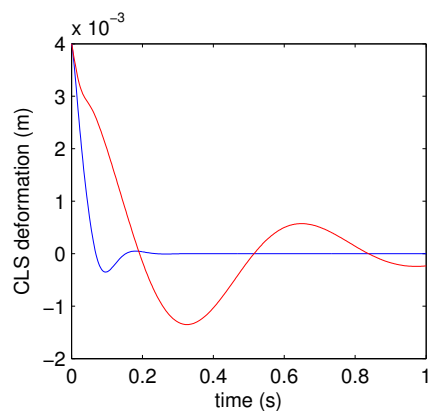

(f) Environment 6

Figure 3.10: CLS deformation results of the uncontrolled (red) and controlled (blue) 1DoF linear system interacting with varying environments. 


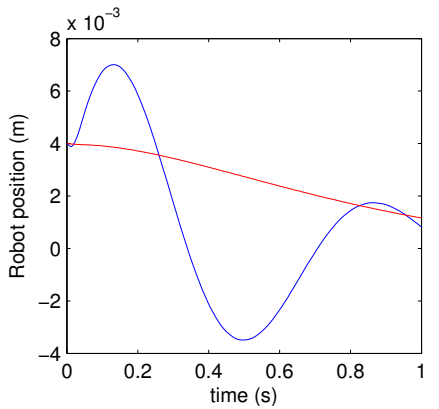

(a) Environment 1

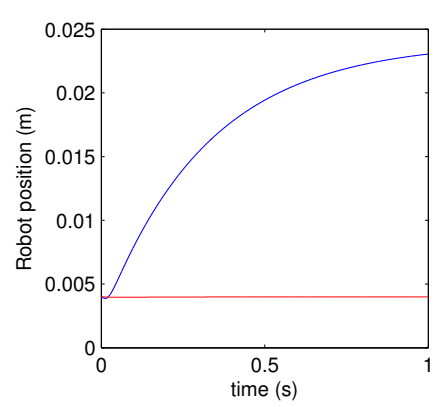

(d) Environment 4

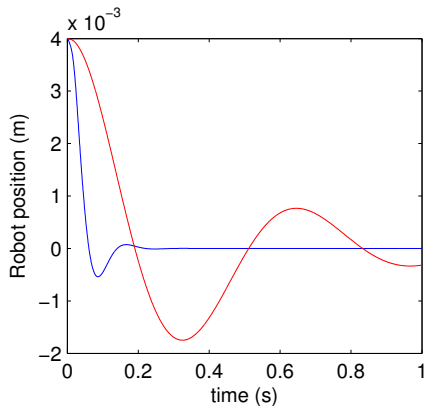

(b) Environment 2

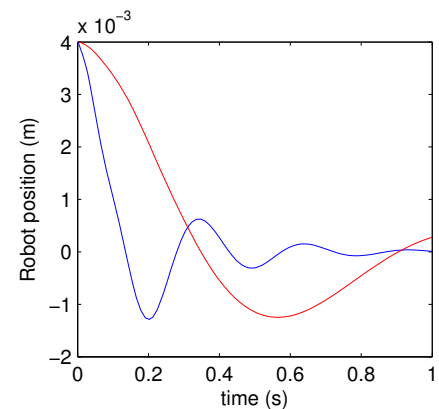

(e) Environment 5

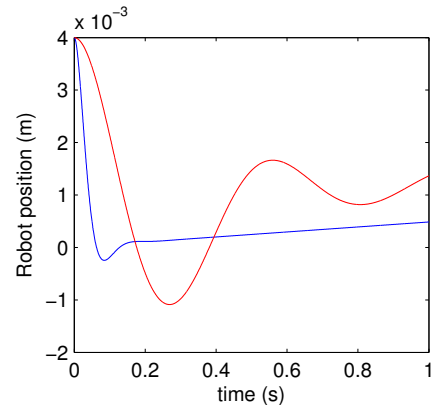

(c) Environment 3

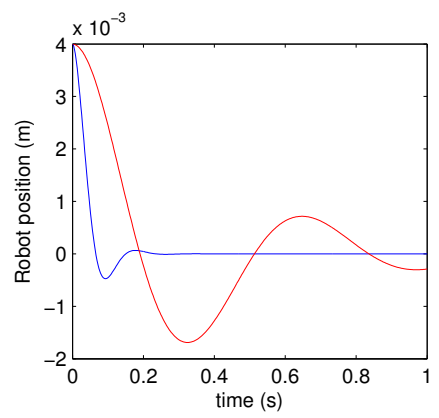

(f) Environment 6

Figure 3.11: Robot motion results of the uncontrolled (red) and controlled (blue) 1 DoF linear system interacting with varying environments.

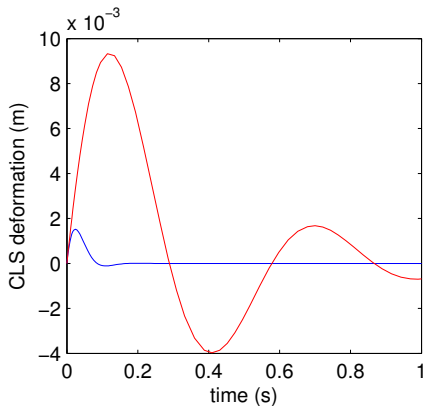

(a) $V_{R}(0)=0.15 \mathrm{~m} \mathrm{~s}^{-1}$

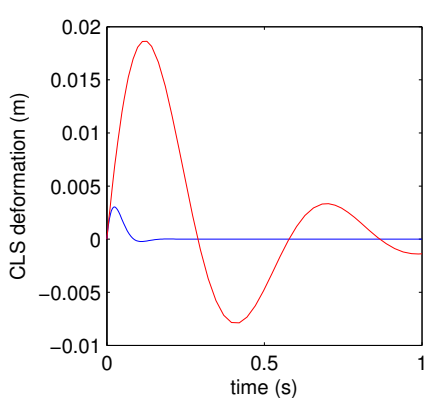

(b) $V_{R}(0)=0.3 \mathrm{~m} \mathrm{~s}^{-1}$

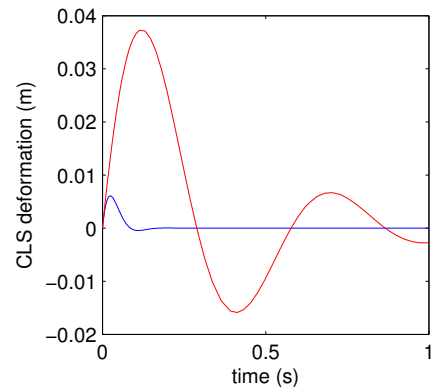

(c) $V_{R}(0)=0.6 \mathrm{~m} \mathrm{~s}^{-1}$

Figure 3.12: CLS deformation results of the uncontrolled (red) and controlled (blue) 1DoF linear system impacting a rigid environment. 


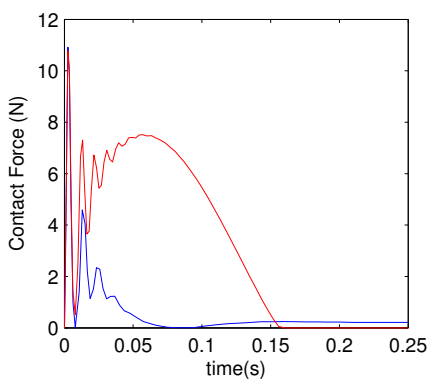

(a) $V_{R}(0)=0.15 \mathrm{~m} \mathrm{~s}^{-1}$

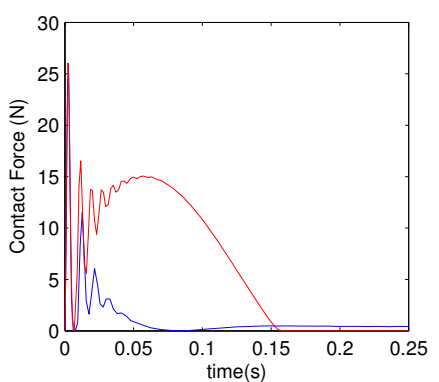

(b) $V_{R}(0)=0.3 \mathrm{~m} \mathrm{~s}^{-1}$

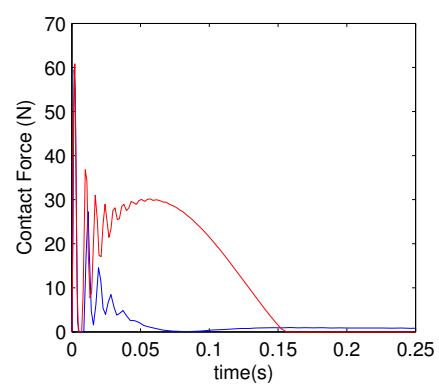

(c) $V_{R}(0)=0.6 \mathrm{~m} \mathrm{~s}^{-1}$

Figure 3.13: Contact forces for $1 \mathrm{DoF}$ linear system, both controlled (blue) and uncontrolled (red) when impacting the employed head model.

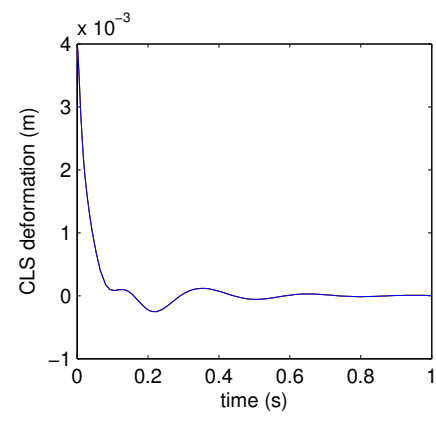

(a) Interaction with Environment 5
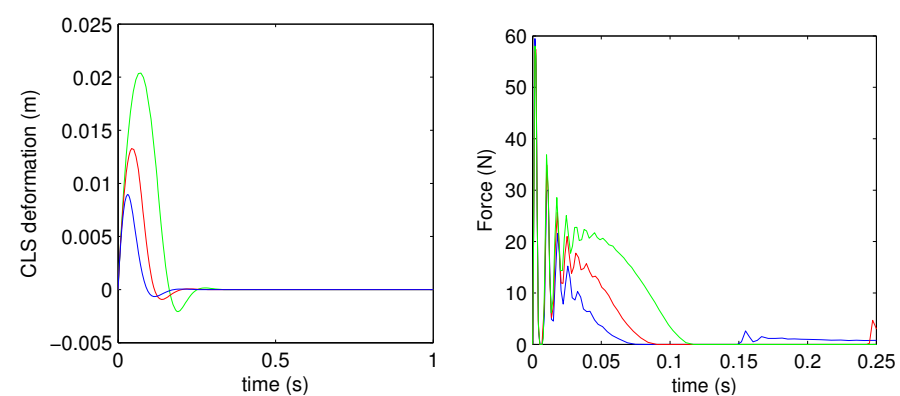

(b) Rigid impact with (c) Head impact with

$$
V_{R}(0)=0.6 \mathrm{~m} \mathrm{~s}^{-1} \quad V_{H}(0)=0.6 \mathrm{~m} \mathrm{~s}^{-1}
$$

Figure 3.14: Sample controller behaviour subject to force saturation at $166.34 \mathrm{~N}$ (blue), $100 \mathrm{~N}$ (red) and $50 \mathrm{~N}$ (green).

As seen in Fig. 3.14, the saturation of actuator force prevents the controller from rapidly responding to collision, causing a build up of contact forces after the initial peak, see Fig. 3.14b and Fig. 3.14c, respectively. 


\subsubsection{DoF linear system with varying sampling frequen- cies}

To simulate the controller with different sampling frequencies, individual time steps are simulated using ode45 and maintaining the actuator force constant. The final CLS deformation value of each time step is then taken as the measurement for that time instance. The actuator force is calculated based on these CLS measurements and a numerically estimated rate of CLS deformation. To determine the effect of changing sampling frequencies, the system was simulated for control at $200 \mathrm{~Hz}, 1 \mathrm{kHz}$ and $2 \mathrm{kHz}$.

From Fig. 3.15, the controller appeared to only change behaviour when operated at $200 \mathrm{~Hz}$, with the $1 \mathrm{kHz}$ and $2 \mathrm{kHz}$ controllers displaying similar responses. The low frequency discrete controller cannot as rapidly respond to impacts with the rigid environment and head model, resulting in larger CLS deformations and contact forces. At $200 \mathrm{~Hz}$, the controller behaviour also begins to deviate from the expected behaviour during interaction, particularly at peaks in the CLS deformation. At lower sampling frequencies, the controller tends toward instability, generating more overshoot and underdamped characteristic in the response.

\subsubsection{DoF linear system with sensor noise}

To simulate the effect of sensor noise on the signal, a noise sample was recorded from the CLS and the noise was added to the measurements of the sampled controller operated at $2 \mathrm{kHz}$. The recorded noise was high pass filtered above $5 \mathrm{~Hz}$ to eliminate DC components of the signal. The noise was also amplified $10 \times$ 


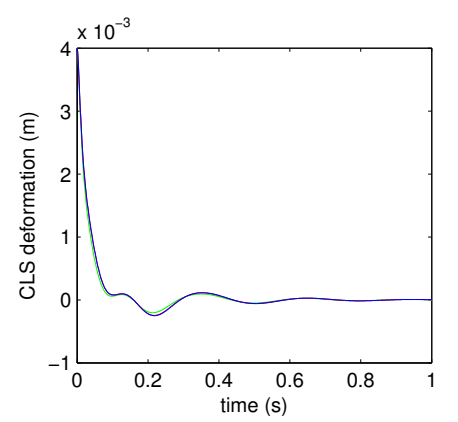

(a) Interaction with Environment 5
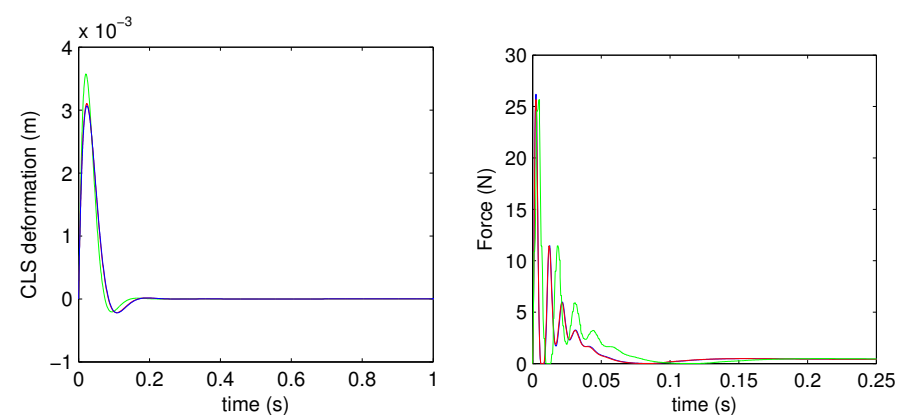

(b) Rigid impact with (c) Head impact with $V_{R}(0)=0.3 \mathrm{~m} \mathrm{~s}^{-1}$
$V_{H}(0)=0.3 \mathrm{~m} \mathrm{~s}^{-1}$

Figure 3.15: Sample controller behaviour subject to discrete control at $2 \mathrm{kHz}$ (blue), $1 \mathrm{kHz}$ (red) and $200 \mathrm{~Hz}$ (green).

and $100 \times$ to determine the effect of increased noise.

The effect of added sensor noise on the behaviour of the controller can be seen in Fig. 3.16. The added noise into the sensor measurements results in small oscillations of the robot motion and CLS deformation with the magnitude of oscillations being proportional to the sensor noise. These oscillations occur due to the controller attempting to follow the sensor noise. Regarding impact of the sensor, noise appears to decrease the responsiveness of the controller, resulting in larger contact forces and greater CLS deformation, see Fig. 3.16b. The motion due to the sensor noise also produces secondary collisions with the head model, see Fig. 3.16c. 


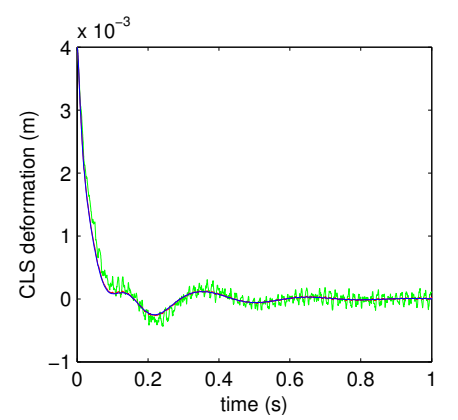

(a) Interaction with Environment 5
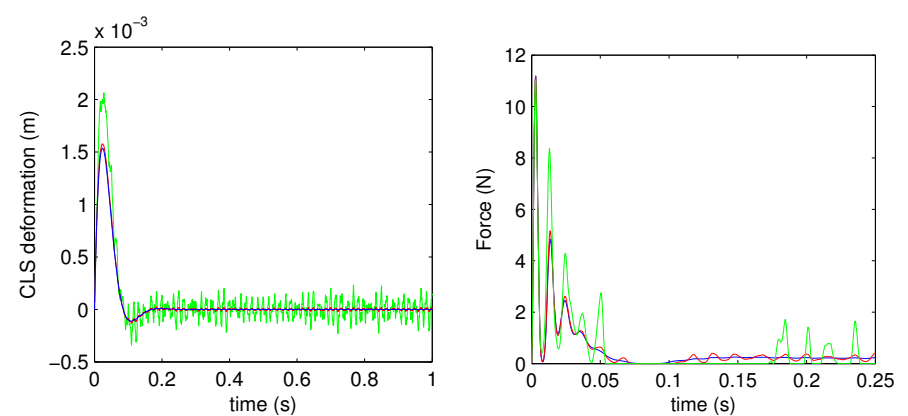

(b) Rigid impact with (c) Head impact with $V_{R}(0)=0.15 \mathrm{~m} \mathrm{~s}^{-1}$ $V_{H}(0)=0.15 \mathrm{~m} \mathrm{~s}^{-1}$

Figure 3.16: Sample controller behaviour subject to discrete control at $2 \mathrm{kHz}$ with the sampled measurement noise (blue), $10 \times$ the measurement noise (red) and $100 \times$ the measurement noise (green).

\subsubsection{DoF linear system with stiction}

Stiction in the CLS dynamics was simulated using the Karnopp model [92] with the nonlinear kinetic friction relationship (provided in Matlab),

$$
F_{f}=-\operatorname{sgn}\left(F_{k}+\left(F_{b}-F_{k}\right) e^{-\tau\left|v_{d}-v_{\text {threshold }}\right|}\right)-B_{S} v
$$

where $F_{k}$ and $F_{b}$ are the kinetic and static friction, and $v_{d}$ is the relative velocity between the CLS shell and the robot. For the current simulations, $F_{k}$ is set to $1 \mathrm{~N}$ less than $F_{b}, \tau$ is set to $4000 \mathrm{~s} \mathrm{~m}^{-1}$ and $v_{\text {threshold }}$ is set to $0.00001 \mathrm{~m} \mathrm{~s}^{-1}$. For the rigid impact simulations, all environment parameters were reduced by two orders of magnitude and ode23s was employed due to the stiff nature of the stiction constraint.

Stiction in the CLS is seen to generate a limit cycle in the CLS deformation when interacting with a fixed environment (see Fig. 3.17a) and results in a non zero steady state robot velocity when interacting with a mass (see Fig. 3.17b). 
The size of the limit cycle and steady state velocity is proportional to the amount of stiction. The limit cycle is believed to occur due to a failing of the stability condition at low velocities as the static friction acts effectively as a large damper, decreasing the ratio $K_{S} / B_{S}$ to below the selected ratio of gains $K_{p} / K_{d}$. This generates an active region close to the origin, which is bounded by passive behaviour, resulting in sustained oscillations [89]. The steady state velocity when interacting with a mass is due to the inability of the CLS to converge to zero deformation due to dry friction. With a steady state offset in CLS deformation, the controller provides a sustained actuation force and the system accelerates until the force due to $B_{R}$ counteracts the force of the actuator. In regards to impact, dry friction increases contact forces proportionally with the magnitude of the friction (see Fig. 3.17d) but does not change the maximum CLS deformation significantly (see Fig. 3.17c).

To eliminate the oscillations and steady state robot velocity caused by stiction, it is proposed to feedback deformation only above a threshold and to set the desired CLS deformation to the threshold value as opposed to 0. By not feeding back small deformations, the robot will not respond to steady state CLS offsets and will not generate active behaviour near the origin. The threshold should be set by considering the behaviour of the CLS and it is proposed to set the largest steady state deformation the CLS can maintain without external forces. The response of the system to a threshold can be seen in Fig. 3.18. The use of a threshold will decrease $V_{d \max }$ and will cause larger contact forces prior to response; hence, a threshold should only be employed if necessary. 


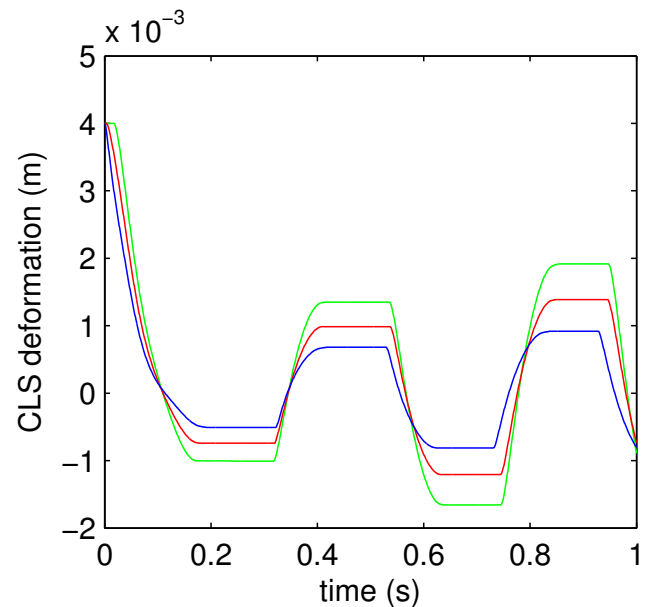

(a) Interaction with Environment 5

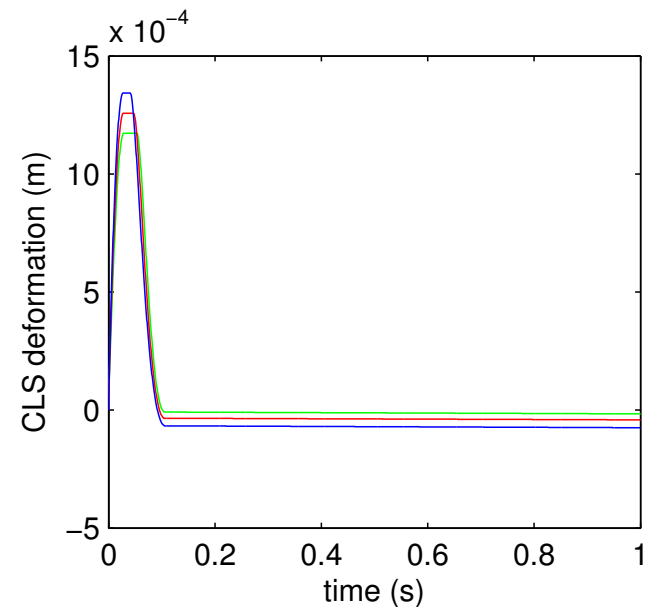

(c) Rigid impact with $V_{R}(0)=($ d $)$ $0.15 \mathrm{~m} \mathrm{~s}^{-1}$

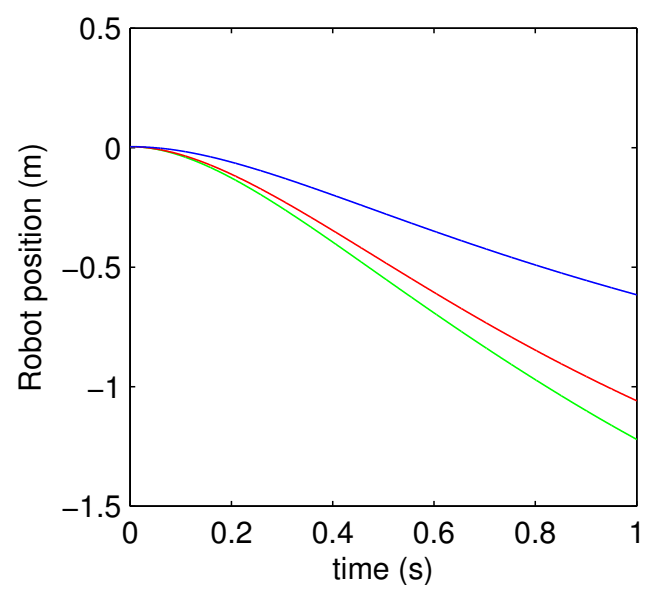

(b) Interaction with Environment 4

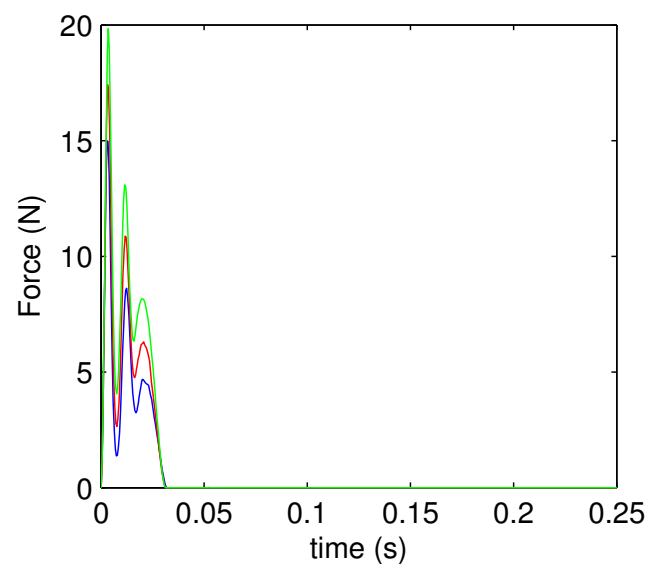

(d) Head impact with $V_{H}(0)=$ $0.15 \mathrm{~m} \mathrm{~s}^{-1}$

Figure 3.17: Sample controller behaviour with the introduction $4 \mathrm{~N}$ (blue), $6 \mathrm{~N}$ (red) and $8 \mathrm{~N}$ (green) of static friction. 


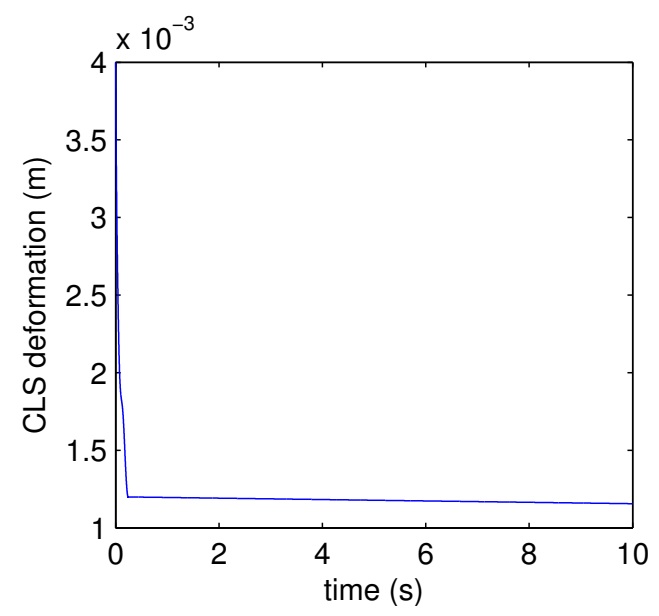

Figure 3.18: System response in interacting with environment 5 with a threshold of $0.0015 \mathrm{~m}$. The CLS stiction was set $4 \mathrm{~N}$.

\subsection{Experimental Validation}

Both the proposed control law and an admittance control law were implemented on the described test platform. The proposed control law used the optimal gains previously determined and the position controller of the admittance controller employed the same $K_{p}$ gain with double the $K_{d}$ gain to prevent overshoot. The desired admittance was a mass-damper model with the inertia reduced to $5 \%$ the original inertia of the test platform and the damping on the model adjusted until stable behaviour was observed $\left(B_{A} \approx 28.52 \mathrm{~N} \mathrm{~s} \mathrm{~m}^{-1}\right)$. The admittance model 
employed in the current work is,

$$
\begin{aligned}
& \overrightarrow{\dot{x}}=A \vec{x}+B F_{\text {ext }} \\
& \text { where } \\
& \vec{x}=\left[\begin{array}{ll}
x_{\text {des }} & \dot{x}_{\text {des }}
\end{array}\right]^{T} \\
& A=\left[\begin{array}{cc}
0 & 1 \\
0 & -\frac{B_{A}}{M_{A}}
\end{array}\right] \\
& B=\left[\begin{array}{cc}
0 & \frac{1}{M_{A}}
\end{array}\right]^{T}
\end{aligned}
$$

where $x_{d e s}$ is the desired position, and $B_{A}$ and $M_{A}$ are the desired damping and inertia of the admittance model. This is a standard admittance model [63] but without the use of a stiffness term. To solve (3.20) and determine the desired position for each time step, the analytical solution of (3.20) is employed with the external force assumed constant across each time step. The discrete implementation of the admittance controller then takes the form [93],

$$
\vec{x}_{k+1}=e^{A T} \vec{x}_{k}+\left(\int_{0}^{T} e^{A \lambda} d \lambda\right) B F_{e x t}
$$

where $T$ is the sampling time of the controller. When the controller is initialized, $\vec{x}_{0}$ is set to the current position and zero velocity. It is noted that the admittance controller is not optimized and the controller is only used to compare general behaviours. Both control laws employ a $1.8 \mathrm{~mm}$ threshold on CLS feedback to prevent the effects of stiction; this threshold is also employed to detect the presence of collision. 


\subsubsection{Impact Testing}

To test the response of the controller to collision, the 1 DoF system was commanded to impact a mounted force sensor at angular velocities of $15^{\circ} \mathrm{s}^{-1}, 30^{\circ} \mathrm{s}^{-1}$ and $40^{\circ} \mathrm{s}^{-1}$. The average maximum impact forces and CLS deformations can be found in Table 3.4 and Table 3.5, respectively. A sample plot of the force and deformation during impact can be found in Fig. 3.19. From Fig. 3.19, it is seen that the sampling frequency of $2 \mathrm{kHz}$ cannot capture the initial peak forces due to the shell inertia. From Table 3.5, it is noted that both controllers prevented the CLS deformation from exceeding $6 \mathrm{~mm}$ which is below the limit of $x_{d \max }=8 \mathrm{~mm}$ set in Sec. 3.3. Both controllers also acted to reduce contact forces by retreating from the impact. The results of the two control laws are comparable, though the admittance controller is observed to decrease forces slightly more at lower impact velocities and to slightly decrease the max CLS deformation over all trials. The improved transient response of the admittance controller compared to the proposed controller opposes the results in literature where deformation feedback has improved transient response over traditional force control when applying a force onto the environment [71]. The differing results from literature is believed

to be due to two factors: 1) the current experiments attempt to apply zero force while the literature attempted to reach a non zero force value and 2) the current experiments employ different position controllers for both control laws while the same position controller is applied to both controllers in the literature. 
Table 3.4: Summarizing statistics of the maximum impact forces over 4 trials for the proposed and admittance controllers during impact at varying angular velocities.

\begin{tabular}{|l|l|l|l|l|l|l|}
\cline { 2 - 7 } \multicolumn{1}{c|}{} & \multicolumn{2}{l|}{$15^{\circ} \mathrm{s}^{-1}$} & \multicolumn{2}{l|}{$30^{\circ} \mathrm{s}^{-1}$} & \multicolumn{2}{l|}{$40^{\circ} \mathrm{s}^{-1}$} \\
\cline { 2 - 7 } \multicolumn{1}{c|}{} & Prop. & Admit. & Prop. & Admit. & Prop. & Admit. \\
\hline $\bar{F}_{\text {max }}(\mathrm{N})$ & 10.88 & 9.83 & 11.90 & 11.98 & 15.03 & 14.97 \\
\hline$s_{F}(\mathrm{~N})$ & 0.1466 & 0.1893 & 0.1613 & 0.1481 & 0.2252 & 0.354 \\
\hline
\end{tabular}

Table 3.5: Summarizing statistics of the maximum CLS deformations over 4 trials for the proposed and admittance controllers during impact at varying angular velocities.

\begin{tabular}{|l|l|l|l|l|l|l|}
\cline { 2 - 7 } \multicolumn{1}{c|}{} & \multicolumn{2}{l|}{$15^{\circ} \mathrm{s}^{-1}$} & \multicolumn{2}{l|}{$30^{\circ} \mathrm{s}^{-1}$} & \multicolumn{2}{l|}{$40^{\circ} \mathrm{s}^{-1}$} \\
\cline { 2 - 7 } \multicolumn{1}{c|}{} & Prop. & Admit. & Prop. & Admit. & Prop. & Admit. \\
\hline $\bar{x}_{\max }(\mathrm{mm})$ & 2.8658 & 2.6801 & 4.3531 & 4.2211 & 5.874 & 5.8 \\
\hline$s_{x}(\mathrm{~mm})$ & 0.0042 & 0.1596 & 0.0535 & 0.1090 & 0.2203 & 0.1218 \\
\hline
\end{tabular}

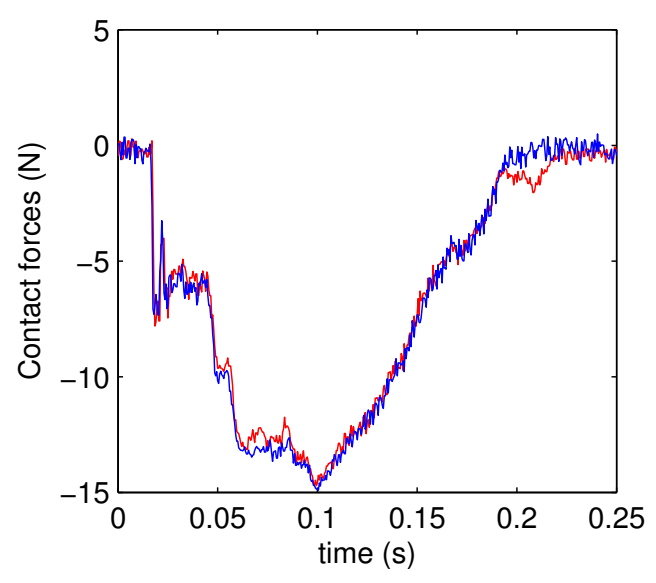

(a)

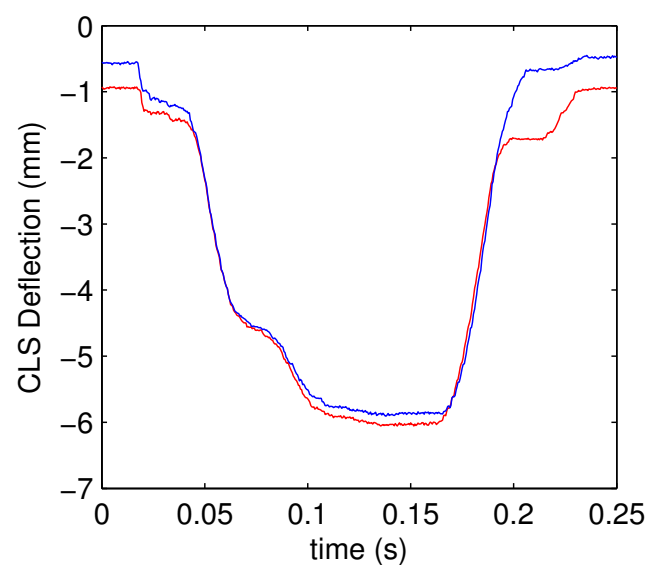

(b)

Figure 3.19: Sample force and deformation measurements for impacts at $40^{\circ} \mathrm{s}^{-1}$ using the admittance (blue) and proposed (red) control laws.

\subsubsection{Interaction Testing}

To test the interaction of the control law with varying environments, the external shell of the CLS was connected to a fixed post through three sets of springs 
with different stiffness, see Table 3.6. The 1DoF system was then commanded to deform the CLS slowly to $4 \mathrm{~mm}$ of deformation and then activate the admittance or proposed SOIS control law. Both compression and tension of the CLS were tested. The CLS deformation over time for each trial can be found in Fig. 3.20 and Fig. 3.21. From the figures, it is seen that both control laws interact in a stable manner with the environments presented and serve to decrease the CLS deformation.

It is noted that the admittance controller decreases the deformation more rapidly than the proposed control law. This rapid decrease is due to the propagation of the admittance model away from the initial point of contact, creating large control errors and commanded torques. Unfortunately, the propagation of the admittance model can move the desired robot position beyond the zero deformation point, resulting in oscillations. These oscillations can result in instability, which was observed in the tuning of the admittance model (see Fig. 3.22).

Table 3.6: Stiffness constants of the tension and compression springs employed to connect the CLS to the fixed post.

\begin{tabular}{|l|l|l|}
\cline { 2 - 3 } \multicolumn{1}{c|}{} & $K_{\text {comp }}$ & $K_{\text {tens }}$ \\
\hline Stiffness 1 & $810 \mathrm{~N} \mathrm{~m}^{-1}$ & $339 \mathrm{~N} \mathrm{~m}^{-1}$ \\
\hline Stiffness 2 & $3345 \mathrm{~N} \mathrm{~m}^{-1}$ & $637 \mathrm{~N} \mathrm{~m}^{-1}$ \\
\hline Stiffness 3 & $9199 \mathrm{~N} \mathrm{~m}^{-1}$ & $979 \mathrm{~N} \mathrm{~m}^{-1}$ \\
\hline
\end{tabular}



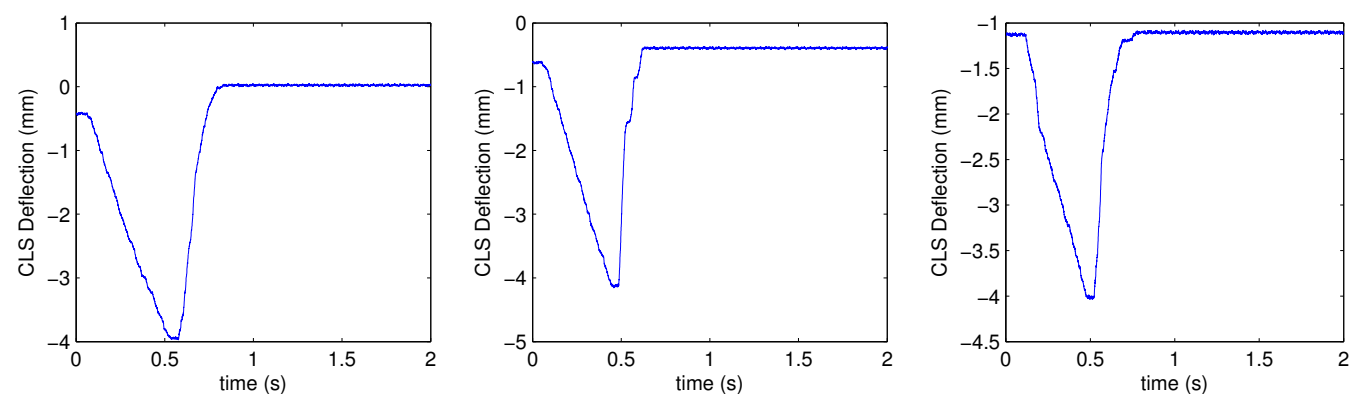

(a) Stiffness 1 with ini- (b) tial tension of the CLS
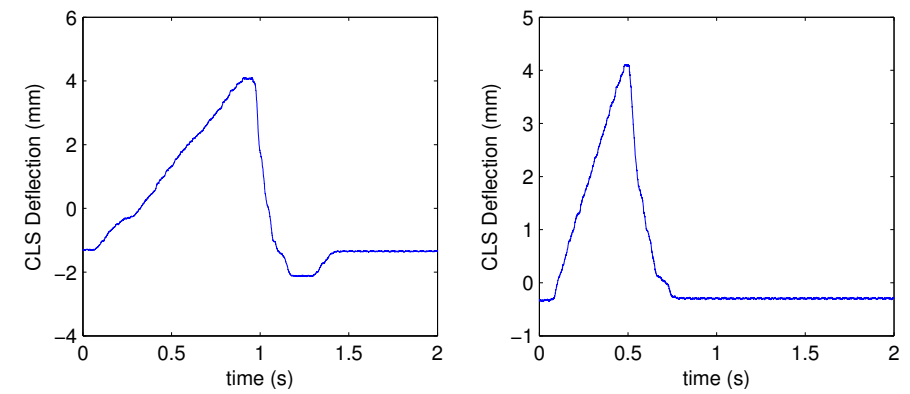

(d) Stiffness 1 with ini- (e) Stiffness 2 with ini-(f) tial compression of the CLS

tial compression of the CLS (c) Stiffness 3 with initial tension of the CLS

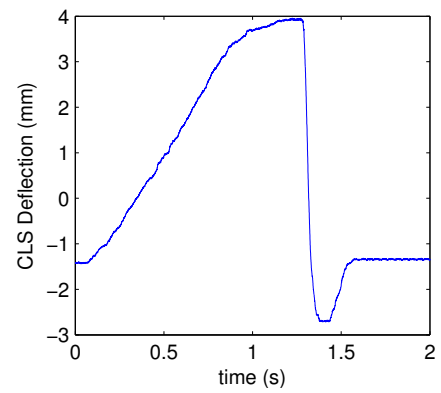

Stiffness 3 with initial compression of the CLS

Figure 3.20: Measured deformation of the CLS during interaction between the environment and the proposed control law.

\subsection{Summary}

A design procedure was developed and the various considerations in implementing the controller were discussed. A 1 DoF hardware set up was characterized and control gains were specified for the system through design procedure. The system and controller were then simulated. Through simulation, it was found that actuator saturation and low sampling rates decrease the ability of the controller to respond to collision. Sensor noise was found to cause oscillations in the CLS deflection, with the size of oscillations being proportional to the magnitude of 

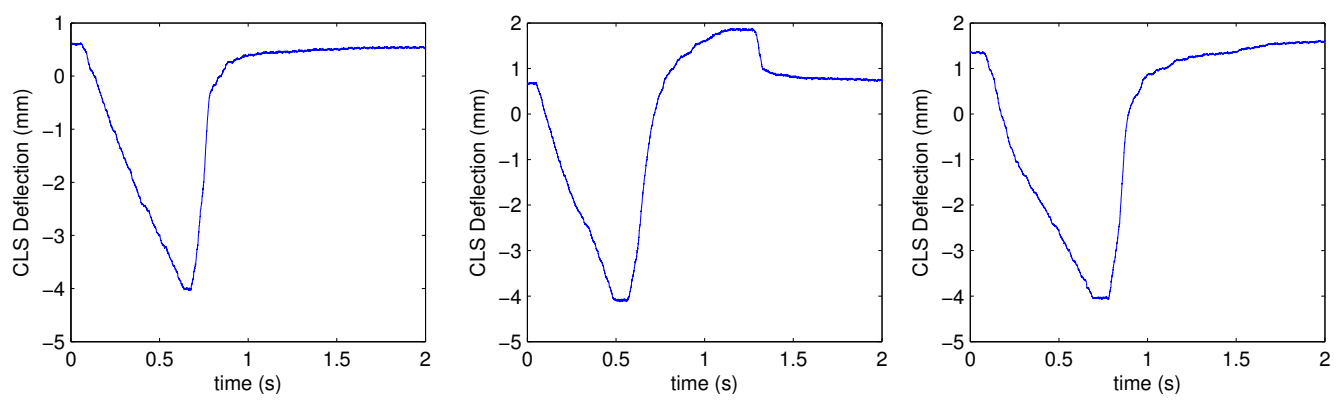

(a) Stiffness 1 with ini- (b) Stiffness 2 with ini-(c) Stiffness 3 with initial tension of the CLS

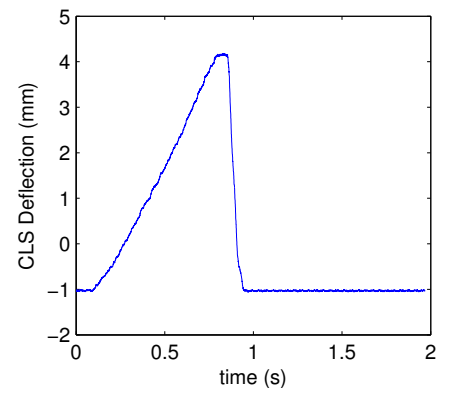
tial tension of the CLS tial tension of the CLS
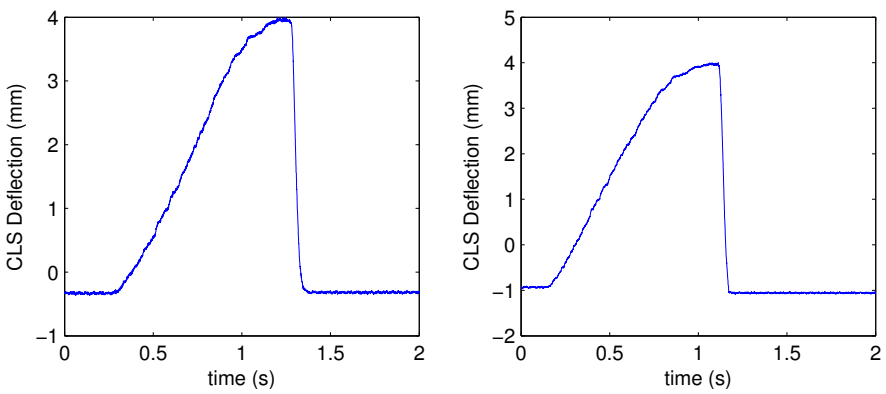

(d) Stiffness 1 with ini- ( tial compression of the CLS

tial compression of the CLS tiffness 3 with initial compression of the CLS

Figure 3.21: Measured deformation of the CLS during interaction between the environment and the admittance control law.

noise. Stiction was simulated in the CLS and found to result in a limit cycle when interacting with a stiff environment and a steady state velocity for inertial environments. To prevent these undesired behaviours, the CLS deformation should only be fed back if the value is above a given threshold. Impact and interaction trials were performed on the 1 DoF test platform with the proposed controller and an admittance control law. In impact, the SOIS controller was found to produce similar reduction in contact force while remaining stable in interaction. 


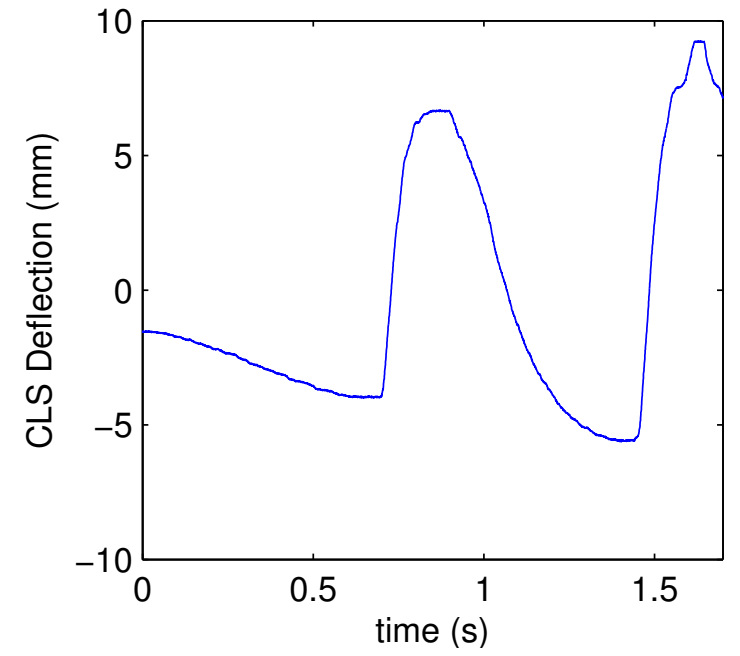

Figure 3.22: Sample unstable behaviour of the admittance controller for reduced damping in the admittance model. 


\section{Chapter 4}

\section{Initial Development of the Three Degree of Freedom Compliant Limb Sensor}

Two planar versions of the CLS are prototyped as an initial effort towards im-

plementing the SOIS on the ViGRR platform. The two planar sensors developed are:

- A potentiometer based CLS: The potentiometer based sensor was developed to facilitate preliminary experiments on the ViGRR and to experimentally determine ideal compliance through impact testing. To be used in preliminary ViGRR experiments, the potentiometer based sensor must provide accurate measurements of the CLS deformation. To determine the ideal compliance experimentally, the ViGRR must perform impact experiments while employing the SOIS controller with varying CLS compliances. To allow such experiments, the stiffness and damping of the CLS must be adjustable to allow a range of CLS dynamics to be investigated.

- An optical based CLS: To eliminate the need for the kinematic chain of the potentiometer based solution, and to better represent the final 
lightweight 3DoF sensor, an advanced optical sensor based CLS was developed in collaboration with other researchers. The optical sensor methodology is evaluated to assess its use for the ViGRR.

Both sensors are designed as a proof of concept research platforms and will not be used as a final solution for the ViGRR. The current work seeks to gain insight for the development of the final planar CLS for the ViGRR. These insights include the sources of error in pose estimation using optical sensing and the limitations of mechanical constraints to ensure planar motion.

\subsection{Potentiometer Based Compliant Limb Sen-}

\section{SOr}

The potentiometer based CLS is a planar sensor capable of measuring $\vec{x}_{d}=$ $[x, z, \theta]^{T}$, where $x$ and $z$ are the motion of the shell in $x$ and $z$ directions, respectively, and $\theta$ is the rotation about the $y$-axis (see Fig. 4.1 for coordinate frame). The sensor was developed to provide independent measurements along each axis and reliable feedback of CLS deformations. To measure $\vec{x}_{d}$ using potentiometers, a three joint, open kinematic chain acted as the connection between the base and external shell. Potentiometers across each joint and a kinematics model can then determine the motion of the external shell with respect to the base. Compliance of the CLS is generated from springs across each joint. Introducing compliance in this way allows the single sensor to display a variety of compliant behaviours by changing the springs, as required to experimentally determine ideal compliance for the final CLS design. In the design, testing and analysis of the potentiometer 


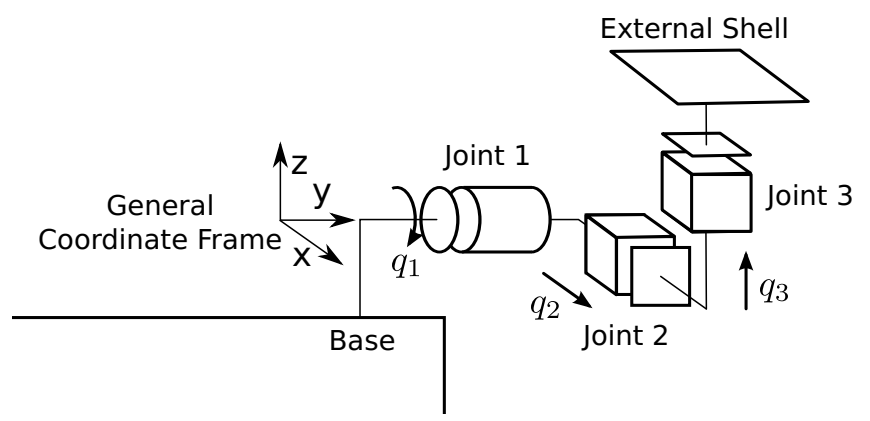

Figure 4.1: Series of joints in the potentiometer based CLS.

based CLS, emphasis was placed on the accurate estimation of the shell motion and the ability of the sensor to be effectively used to evaluate the proposed control methodology.

\subsubsection{Mechanical Design}

The series of joints chosen for the potentiometer based CLS are, from the base to the external shell, a single revolute joint followed by two perpendicular prismatic joints, see Fig. 4.1. The proposed kinematics are accomplished using a ball bearing revolute joint and two linear guides, as shown in Fig. 4.2. The proposed series of joints increases the overall mass of the CLS by using two linear guides; however, the design offers advantages through the simple kinematics of the joints to facilitate ViGRR experiments.

\subsubsection{CLS Kinematics}

In implementing the proposed SOIS control law for the planar case with the potentiometer based CLS, the goal of the controller is to specify the motion of the CLS base on the robot $\left(\vec{x}_{\text {base }}\right)$ such that the joint positions of the CLS reach 


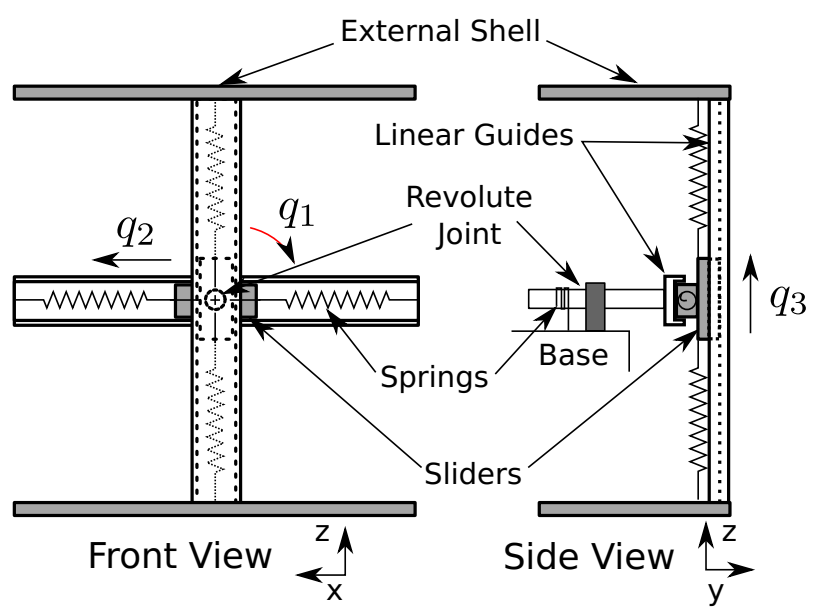

Figure 4.2: The implementation of the open loop kinematic chain for the potentiometer based CLS.

zero $(\vec{q}=0)$. In specifying this motion, care must be taken to ensure that the commanded velocities, $\overrightarrow{\dot{x}}_{\text {base }}$, do not result in increasing the magnitude of joint positions for any point of the trajectory. Increasing the magnitude of joint positions will result in increased forces across the joints and possible increases in contact forces.

The current arrangement of joints was chosen to decouple the relationship between the movement of the base with respect to the external shell and the CLS joint displacements. This decoupling can be seen in the following relationship between the velocity of each joint of the CLS and the velocity of the robot at the 
base of the sensor with respect to the external shell that is assumed to be fixed,

$$
\begin{aligned}
& \overrightarrow{\dot{x}}_{\text {base }}=J_{C L S} \overrightarrow{\dot{q}} \\
& {\left[\begin{array}{c}
\dot{x} \\
\dot{z} \\
\omega_{y}
\end{array}\right]=\left[\begin{array}{lll}
0 & 1 & 0 \\
0 & 0 & 1 \\
1 & 0 & 0
\end{array}\right]\left[\begin{array}{l}
\dot{q}_{1} \\
\dot{q}_{2} \\
\dot{q}_{3}
\end{array}\right]}
\end{aligned}
$$

where $\overrightarrow{\dot{q}}$ is the vector of CLS joint velocities, and $J_{C L S}$ is the Jacobian relating the two velocities.

The decoupling seen in (4.1) directly affects the implementation of the control law by relaxing requirements on the generation of trajectories to reach zero deformation, $\vec{x}_{d} \rightarrow 0$. The decoupled kinematics observed for the current sensor directly relate each component of $\vec{x}_{b a s e}$ to a joint velocity. Consequently, any robot trajectory that continually moves the base toward $\vec{x}_{d}=0$ in Cartesian space, will also continually decrease the CLS joint deflections, $\vec{q}$. If the kinematics were not decoupled, the Jacobian of the base of the CLS with respect to the external shell would need to be considered at each time step when generating the trajectory, complicating the implementation of the control law.

The use of an open kinematic chain also allows for large movements of the CLS, which are useful in preliminary implementation of the SOIS control law. With larger commanded motions, the behaviour of the SOIS controller can be viewed over longer time periods, assisting in characterizing the interactions between the ViGRR and the environment. The larger deformations will also help prevent the complete compression of the CLS in testing. During testing, the complete 
compression of the CLS can cause large contact forces, potentially damaging equipment, and must be avoided.

\subsubsection{CLS Dynamics}

The dynamics for the current design were developed using the Lagrange equations [94] considering only planar motions. In deriving the equations, it is assumed the axes of all joints are perpendicular, the three axes intersect at a point when $\vec{q}=\overrightarrow{0}$, friction is viscous and each axis passes through the centre of mass of the associated link. Under these assumptions, the dynamics of the CLS are represented by the equation,

$$
D\left(\vec{q}_{C L S}\right) \overrightarrow{\ddot{q}}_{C L S}+C\left(\vec{q}_{C L S}, \overrightarrow{\dot{q}}_{C L S}\right) \overrightarrow{\dot{q}}_{C L S}+B \overrightarrow{\dot{q}}_{C L S}+K \vec{q}_{C L S}+G\left(\vec{q}_{C L S}\right)=\tau
$$


where $\tau$ are the externally applied loads on each joint, and,

$$
\begin{aligned}
& D\left(\vec{q}_{C L S}\right)=\left[\begin{array}{ccc}
I_{1}+I_{2}+I_{3}+M_{2} q_{2}^{2}+M_{3} q_{2}^{2}+M_{3} q_{3}^{2} & -M_{3} q_{3} & M_{3} q_{2} \\
-M_{3} q_{3} & M_{2}+M_{3} & 0 \\
M_{3} q_{2} & 0 & M_{3}
\end{array}\right] \\
& C\left(\vec{q}_{C L S}, \overrightarrow{\dot{q}}_{C L S}\right)=\left[\begin{array}{ccc}
\dot{q}_{2} q_{2}\left(M_{2}+M_{3}\right)+\dot{q}_{3} M_{3} q_{3} & \dot{q}_{1} q_{2}\left(M_{2}+M_{3}\right) & \dot{q}_{1} M_{3} q_{3} \\
-\dot{q}_{3} M_{3}-\dot{q}_{1} q_{2}\left(M_{2}+M_{3}\right) & 0 & -\dot{q}_{1} M_{3} \\
M_{3}\left(\dot{q}_{2}-\dot{q}_{1} q_{3}\right) & \dot{q}_{1} M_{3} & 0
\end{array}\right] \\
& B=\left[\begin{array}{ccc}
B_{1} & 0 & 0 \\
0 & B_{2} & 0 \\
0 & 0 & B_{3}
\end{array}\right] \\
& K=\left[\begin{array}{ccc}
K_{1} & 0 & 0 \\
0 & K_{2} & 0 \\
0 & 0 & K_{3}
\end{array}\right] \\
& G(\vec{q})=\left[\begin{array}{c}
-M_{3} q_{3} g \sin \left(q_{1}\right)+M_{2} q_{2} g \cos \left(q_{1}\right)+M_{3} g q_{2} \cos \left(q_{1}\right) \\
M_{2} g \sin \left(q_{1}\right)+M_{3} g \sin \left(q_{1}\right) \\
M_{3} \cos \left(q_{1}\right) g
\end{array}\right]
\end{aligned}
$$


In (4.3)-(4.7), $M_{i}$ and $I_{i}$ are the mass and second moment of inertia of link $i$ in the plane, respectively, $B_{i}$ and $K_{i}$ are the damping and spring constants of joint $i$, respectively, and $g$ is the acceleration due to gravity. Equations (4.2)-(4.7) show the highly coupled nature of the dynamics of each joint. The equations of motion presented above must be used in the analysis of the stability and performance of the proposed control law; the coupled nature of the equations greatly complicates the analysis for the 3DoF control law.

\subsubsection{Hardware}

The described sensor design was implemented to test the ability of potentiometers to determine external shell pose for the given configuration, see Fig. 4.3. A miniature stainless steel ball bearing acted as the revolute joint and two Igus NW-02-17 plastic sliders acted as the prismatic joints. All selected joints are low mass. The stainless steel ball bearing offers the strength required for the base joint with low friction. In experiments to test the effect of CLS compliance on injury, friction could be added by adding a damper to the joint axle. The plastic linear guides also offer adjustable friction through the potential use of preloaded plastic sliders and lubricant.

For the current implementation, springs were placed across each joint to allow the CLS to return to a rest position after the external shell was moved. The springs chosen were low stiffness to allow the external shell to be moved easily by hand while evaluating the accuracy of the sensor's pose estimation. The addition of the springs also revealed potential difficulties in employing this design such as 


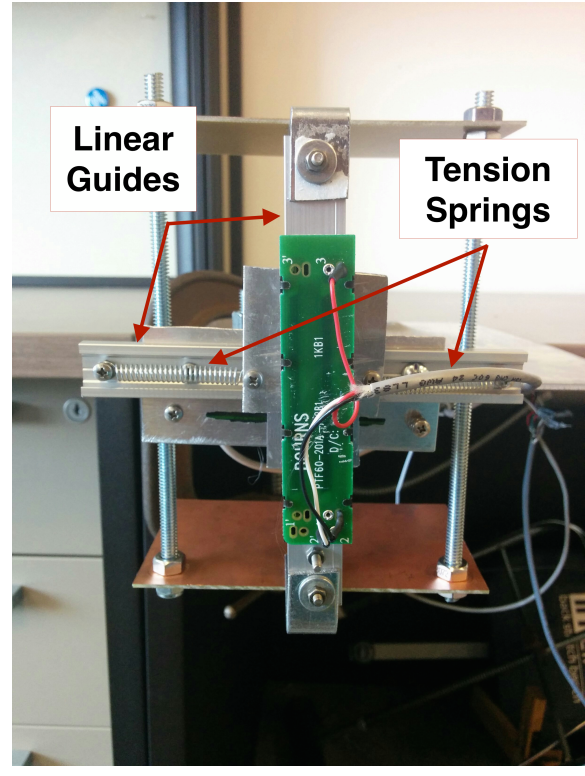

(a)

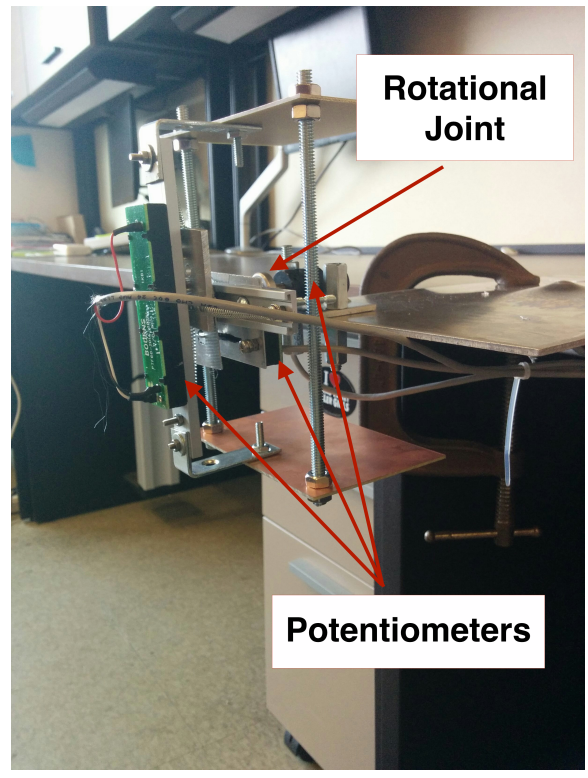

(b)

Figure 4.3: Prototype of the potentiometer based CLS: a) front view and b) side view.

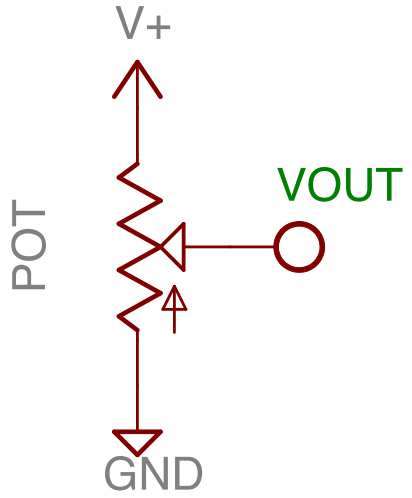

Figure 4.4: Circuit used for potentiometer measurement. VOUT is measured using a PCI 6229 data acquisition card and $\mathrm{V}+$ is set to $9.7 \mathrm{~V}$. 
locking of the linear sliders.

To measure the motion of each joint, the current design employs a CT3034$\mathrm{ND}$ precision $5 \mathrm{k} \Omega$ rotary potentiometer to measure rotation of joint 1 and two PTF60-201A-102B1-ND $1 \mathrm{k} \Omega$ sliding potentiometers to measure displacements of joints 2 and 3. The potentiometer circuit used for all potentiometers can be seen in Fig. 4.4. The rotary potentiometer was chosen for its precision resistance, allowing for more accurate measurement of the relatively small rotations expected. The sliding potentiometers were chosen to provide a large range of motion $(60 \mathrm{~mm})$ and low resistance to minimize noise from the ViGRR motors. While meeting these requirements, the current sliding potentiometers are relatively large and increase the mass of the external shell.

\subsubsection{Sensor Calibration}

The potentiometer based CLS was calibrated to determine a mapping between the measured potentiometer voltages and the motion of the external shell. To determine this mapping, the orientations of the joint axes are calibrated to allow the transformation of the external shell relative to the base to be determined from known joint positions, $q$. The potentiometers are then calibrated to determine the relationship between measured voltage and the joint positions.

\subsubsection{Experimental Setup}

To calibrate the potentiometer based CLS, the sensor was moved by hand along three trajectories: rotation, translation in the $x$ direction and translation in the $z$ direction. These trajectories relate primarily to motion of joints $1-3$, respectively. 
Motion of the CLS was captured using an NDI Optotrak Certus 3D motion capture system. A 6DoF marker was placed on each link and motion was captured at a rate of $100 \mathrm{~Hz}$. The output of the potentiometers and the supply voltage were measured at $2 \mathrm{kHz}$ using an NI PCI 6229 DAQ card connected to a real time PC programmed using the Simulink Real Time toolbox. The Optotrak motion data and the measured voltages were synchronized by measuring the external trigger of the Optotrak.

\subsubsection{Joint Axes Calibration}

To relate the joint positions to the motion of the external shell with respect to the base, the orientations of the joint axes were calibrated. The location and orientation of the axis of rotation for the ball bearing were determined using the data from the rotation trial. The orientation of the axis of rotation was determined by fitting the motion of the marker on the first link to a plane by minimizing the sum of squared error. The axis of rotation was then determined as the normal of the plane. To determine the location of the axis of rotation, the motion of the first marker was projected onto the previously determined plane and a circle was fit to the data, using the algebraic procedure described in [95]. The axis of the first prismatic joint was determined by a least orthogonal distance line of best fit for the motion of the second link relative to the first during the $x$ translation trial. The equations employed to determine the line of best fit can be found in [96]. The second prismatic joint was fit in the same manner using the motion of the third link relative to the second in the $z$ translation trial.

A general coordinate frame was determined with the origin at the axis of rotation (see general coordinate frame in Fig. 4.2). A rest position was then selected 
Table 4.1: Components of joint axes in the general coordinate frame for the rest position.

\begin{tabular}{|l|lll|}
\hline Axis & $x$ & $y$ & $z$ \\
\hline Joint 1 & 0.0291 & 0.9996 & -0.006 \\
\hline Joint 2 & 1 & 0 & 0 \\
\hline Joint 3 & -0.0508 & 0.0269 & 0.9984 \\
\hline
\end{tabular}

from the data and all joint axes were transformed into the general coordinate frame for this position (see Table 4.1). The motion of a point on the external shell can then be estimated from the joint positions using the equation,

$$
P=R_{\text {joint } 1}\left(q_{1}\right) T_{\text {joint } 2}\left(q_{2}\right) T_{\text {joint } 3}\left(q_{3}\right) P_{\text {rest }}
$$

where $P$ and $P_{\text {rest }}$ are the new location of the point and the rest position, respectively, $q_{i}$ is the deviation of the position of joint $i$ from the rest position, $R_{\text {joint } 1}\left(q_{1}\right)$ is the rotation matrix about the axis of joint 1 by $q_{1}$, and $T_{\text {jointi }}\left(q_{i}\right)$ is the translation matrix along the joint axis $i$ by $q_{i}$.

\subsubsection{Potentiometer Calibration}

With the joint axes determined, the potentiometers are calibrated to determine the joint positions from the measured voltages. The sliding potentiometers were calibrated by linear regression of the measured voltage ratio against the measured displacement along the joint axis. The potentiometer of joint 2 and joint 3 were calibrated using the $x$ translation and $z$ translation trials, respectively. The rotary potentiometer was calibrated using the rotation trial. The relationship between the voltage ratio and rotation was selected to minimize the squared error between 
Table 4.2: Factor to convert from changes in voltage ratio to joint displacements for each link

\begin{tabular}{|l|l|}
\hline Potentiometer & Slope \\
\hline Joint 1 & $213.883^{\circ}$ \\
\hline Joint 2 & $-53.8415 \mathrm{~mm}$ \\
\hline Joint 3 & $53.2922 \mathrm{~mm}$ \\
\hline
\end{tabular}

the observed motion of a point on the third link and the estimated motion. The slopes for the conversion from voltage ratio to motion can be found in Table 4.2.

\subsubsection{Performance}

\subsubsection{Procedure}

To test the performance of the potentiometer based CLS in tracking the motion of the plane of the external shell, four new trajectories were recorded using the setup described above with the CLS undergoing general motion. For the trials, the location of the origin and the orientation of the $x$-axis of a coordinate frame on the third link was measured using the Optotrack system and estimated using the potentiometer based CLS. Paths of the measured and estimated motion can be found in Fig. 4.5.

\subsubsection{Results}

Summarizing statistics outlining the accuracy of the sensor in determining position and orientation can be seen in Table 4.3. More detailed analysis of errors in tracking the position and orientation in the $x-z$ plane can be found in Table 4.4. 

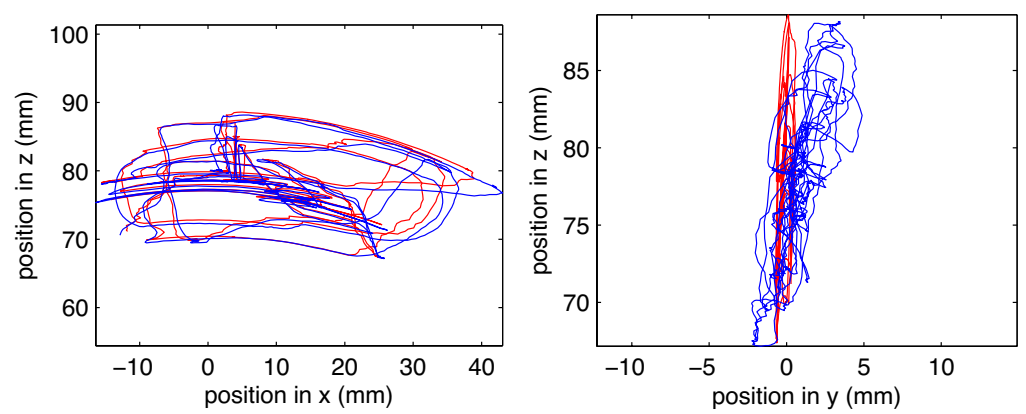

(a) Trial 1
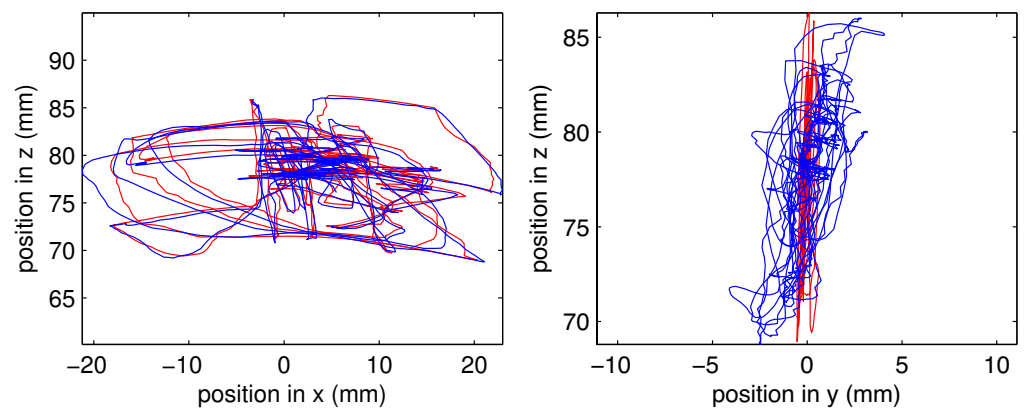

(b) Trial 2
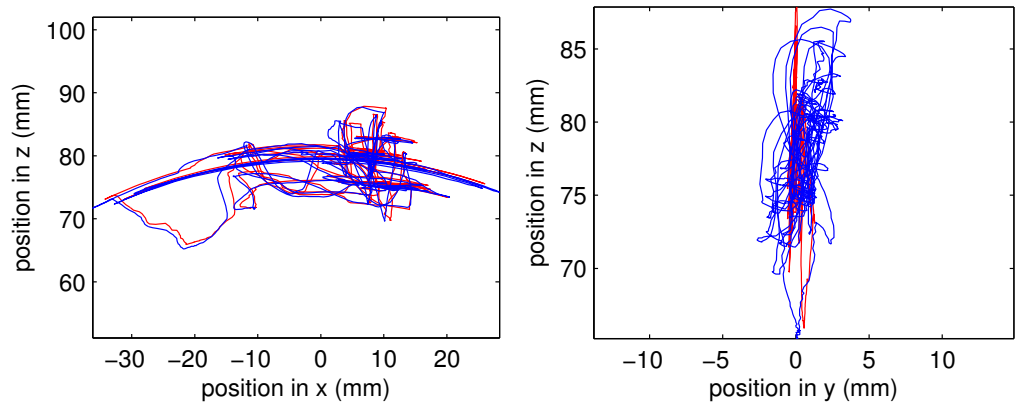

(c) Trial 3
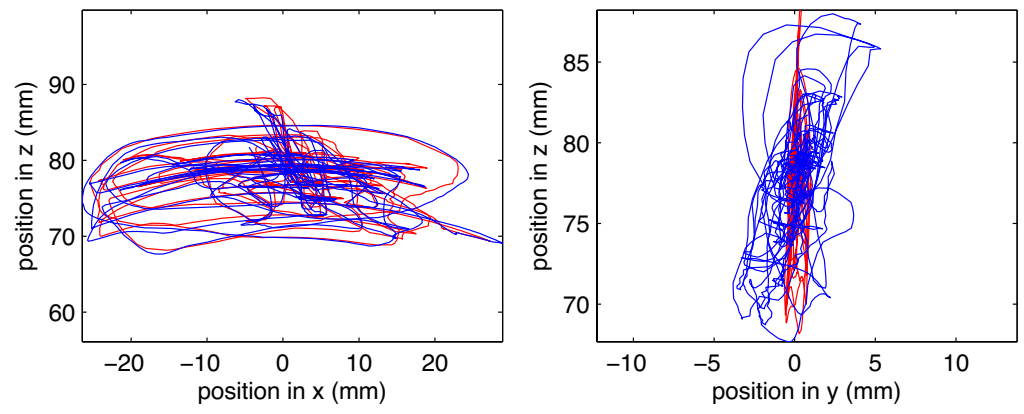

(d) Trial 4

Figure 4.5: Paths of the measured (blue) and estimated (red) motion of the point in $3 \mathrm{D}$ space. 
Table 4.3: Summarizing statistics on the accuracy of the potentiometer based CLS to track motion and orientation of the external shell.

\begin{tabular}{|l|l|l|}
\cline { 2 - 3 } \multicolumn{1}{c|}{} & $\bar{e}$ & $s_{e}$ \\
\hline Error & $1.5306 \mathrm{~mm}$ & $0.8851 \mathrm{~mm}$ \\
\hline Percent Error & $4.98 \%$ & $2.51 \%$ \\
\hline Error in $x-z$ plane & $0.9169 \mathrm{~mm}$ & $0.6515 \mathrm{~mm}$ \\
\hline Percent Error in $x-z$ plane & $2.97 \%$ & $1.82 \%$ \\
\hline Orientation Error & $0.9598^{\circ}$ & $0.5924^{\circ}$ \\
\hline Orientation Error in Plane & $0.498^{\circ}$ & $0.43^{\circ}$ \\
\hline
\end{tabular}

Table 4.4: Summarizing statistics of errors in tracking motion and orientation in the $x-z$ plane

\begin{tabular}{|l|l|l|l|}
\cline { 2 - 4 } \multicolumn{1}{c|}{} & $\bar{e}$ & $s_{e}$ & $\max (e)$ \\
\hline Magnitude of error in X & $0.8047 \mathrm{~mm}$ & $0.6428 \mathrm{~mm}$ & $4.3741 \mathrm{~mm}$ \\
\hline Magnitude of error in Z & $0.3235 \mathrm{~mm}$ & $0.316 \mathrm{~mm}$ & $2.9286 \mathrm{~mm}$ \\
\hline Magnitude of error in $\theta$ & $0.498^{\circ}$ & $0.43^{\circ}$ & $3.2540^{\circ}$ \\
\hline
\end{tabular}

The error in the $x-z$ plane is of great concern as the ViGRR can only respond to this motion. The measured and estimated motion and orientation in the $x-z$ plane over time can be found in Fig. 4.6.

\subsubsection{Discussion}

\subsubsection{Mechanical Design}

The need for a kinematic chain connecting the external shell to the base is the main challenge to implementing the potentiometer based CLS. Even designed 

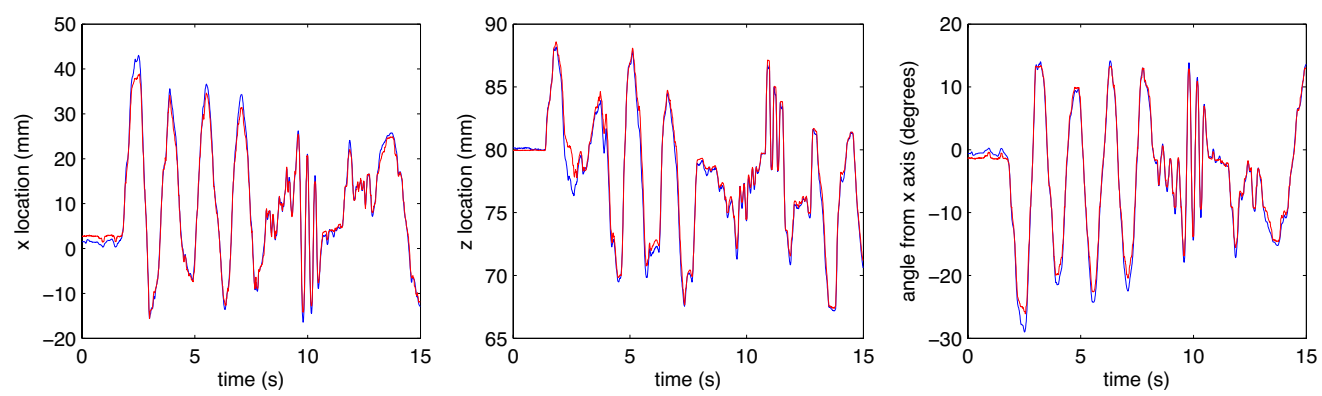

(a) Trial 1
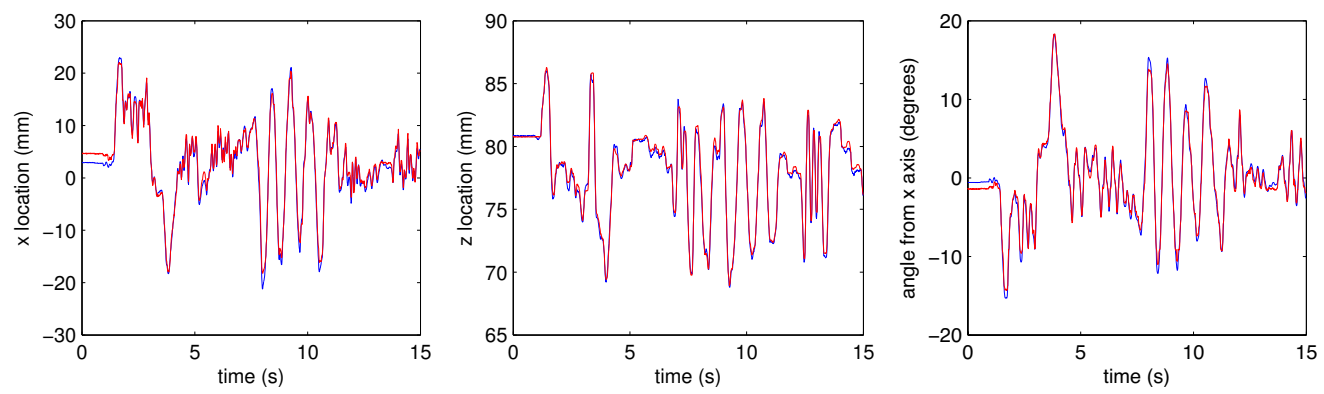

(b) Trial 2
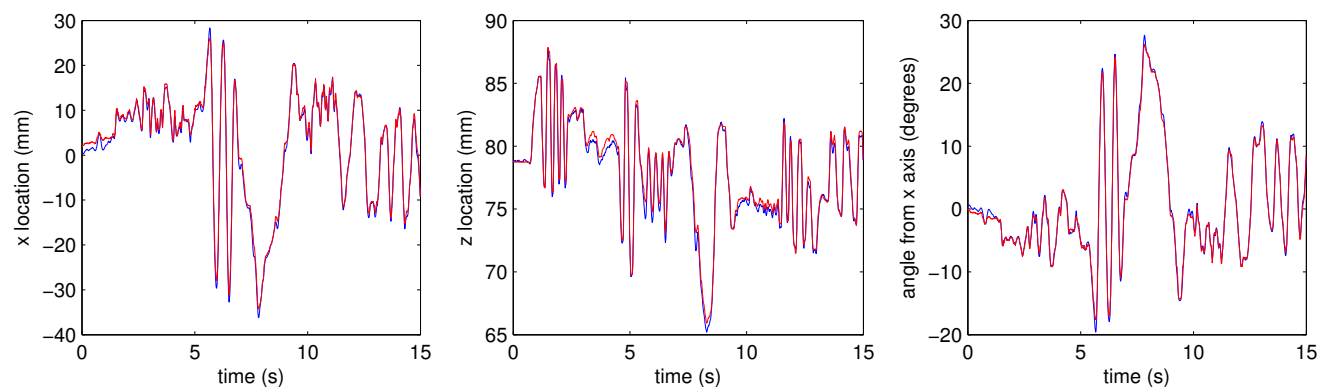

(c) Trial 3
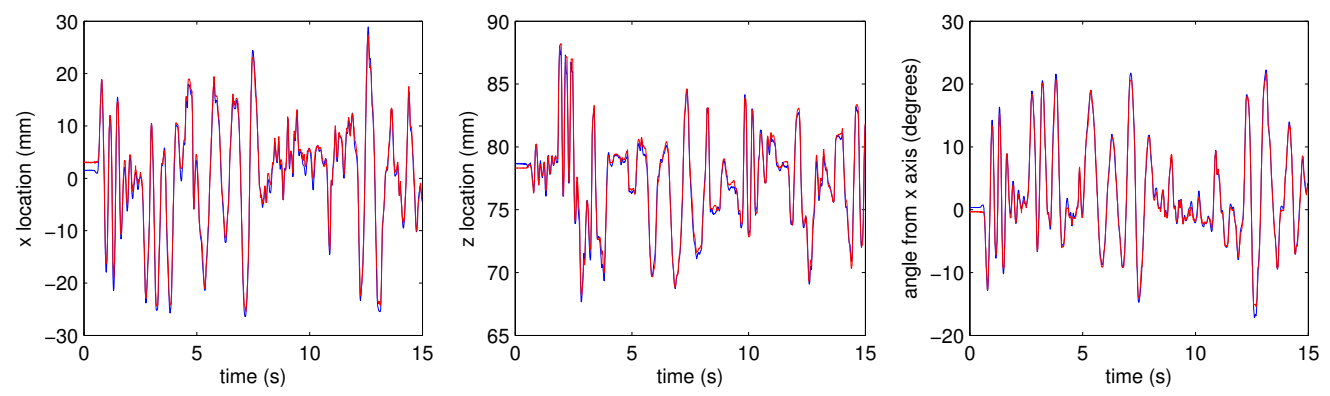

(d) Trial 4

Figure 4.6: Measured (blue) and estimated (red) motion and orientations in the $x-z$ plane. 
with the goal of minimizing inertia, the current prototype is heavier than desired. In addition, the kinematic chain requires space and so results in large sensors, greatly increasing the size of the link should the sensor be attached to a robot. These difficulties can be reduced by changing the types of joints employed, notably by using three rotational joints; however, different joints may result in complex kinematics and dynamics that would need to be accounted for in the controller implementation. Despite these deficits, the current mechanical design of the CLS allows a relatively large range of motion, and has simple kinematics and adjustable compliance, providing a suitable research platform for the proposed experiments.

\subsubsection{Sensor Performance}

The potentiometer based solution has demonstrated the ability to determine the location and orientation of an external shell with minimal computation. The ability of the CLS to track deformation can be seen in Fig. 4.6. The mean error in the $x-z$ plane is less than $1 \mathrm{~mm}$, representing a mean percent error of $2.97 \%$. These errors are below the mean error of the ViGRR's end effector accuracy (2.8576 mm) and on the same order as the position controller's repeatability [81]. These results indicate that the potentiometer based CLS can be used for early experiments employing the SOIS control methodology for the planar case. However, from Table 4.4, the maximum errors are large. If such errors were fed back into the control law, the controller performance would be negatively affected. Based on the simulations of sensor noise for the 1DoF system, errors would result in oscillations of the robot position and contact forces. The exact effects of such errors for the $3 \mathrm{DoF}$ case would require a more thorough analysis. Fortunately, such large errors are rare, as indicated by the low standard deviation for the errors of all 
components in the plane.

The errors in estimating the location and orientation of the external shell most likely originate from undesired motion in the mechanical connections and errors in calibrating the joint axes. Some relative motion exists between components in the kinematic chain. These motions result in displacements in the $y$ direction, as seen in Fig. 4.5. These out of plane motions, both translations and rotations, cannot be estimated using the potentiometers and so result in errors. In addition, these motions would exist in the data used for calibration, resulting in incorrect joint axes that affect all subsequent estimates. Reducing this relative motion would require tightening all connections, which would increase the friction in the CLS. Such increases of friction can cause locking of the plastic linear bearings.

\subsection{Optical Sensor Based Compliant Limb Sen- SOr}

This section is a summary of the development of the optical sensor, with emphasis placed on the contributions of the author. The work has been a collaborative effort with Kyle Chisholm and Jennifer Baba of the ABL lab group with the goal of developing a CLS without the need for the open kinematic chain required for the potentiometer based solution. The development of the sensor is outlined in a paper currently under revision under the title "A Limb Compliant Sensing Strategy for Robot Collision Reaction". The main contributions of the author was in the sensor design, the fitting of an approximate model to the light-to-voltage (LTV) sensor, and analysis of results. Specifically the author: 
- Proposed the LTV sensor model.

- Fit the observed LTV sensor voltages to the sensor model.

- Proposed the placement of the LEDs and LTV sensors.

- Assessed the potential of the sensor to be employed with the SOIS control law.

The initial experiments characterizing the light-to-voltage sensor response and the foam characteristics were performed by Jennifer Baba and the overall sensor model and the numerical methods to calibrate the sensor and solve for pose were done by Kyle Chisholm.

\subsubsection{Sensor Design}

The optical sensor based CLS employs LED/LTV sensor pairs to estimate the motion of an external shell compliantly linked to the base. A schematic and labelled photo of the current 3DoF planar prototype can be seen in Fig. 4.7. For the current design, two LEDs are attached to the external shell with the light emitted from each being sensed by clusters of two or three LTV sensors on the base. All LEDs and LTV sensors are in the plane in which the CLS seeks to observe motion. Motion of the external shell will adjust the light sensed by the LTV sensors, affecting their output. Using a model for the interaction between the LED and LTV sensors, the pose can then be estimated.

The compliant connection between the external shell and the base was achieved using three columns of foam with a cross sectional area of $1.5 \mathrm{in}^{2}$ square and a height of 1.5 in. Each column was composed of two layers of soft natural gum 


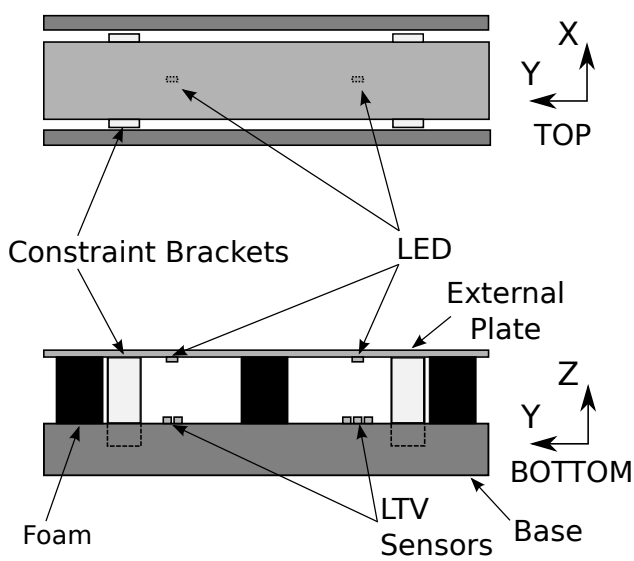

(a)

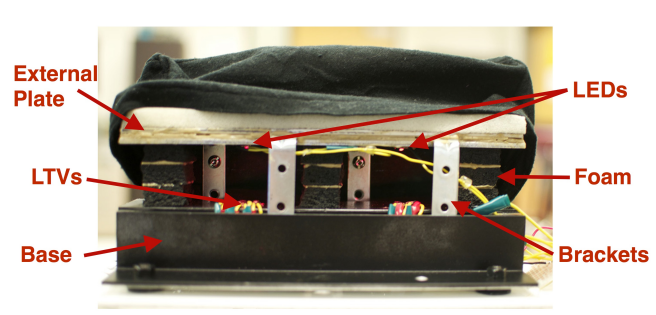

(b)

Figure 4.7: Overview of the optical sensor based CLS: a) Schematic of prototype, b) labelled photograph of constructed prototype.

foam and one extra soft natural gum foam. Brackets, attached to the external shell, were fit into slots on the base of the CLS. The brackets and slots formed a kinematic constraint to prevent motion outside of the plane of interest. The entire sensor was then covered by fabric to prevent ambient light from affecting the LTV sensors' outputs.

\subsubsection{LTV sensor modelling}

\subsubsection{Proposed Model}

To develop a model of the LTV sensor response to the light emitted by the LED, the following simplifying assumptions were made:

- The light from the LED diffuses as a sphere.

- The amount of light emitted from the LED in a given direction from its center would be proportional to the area of the emitter seen from that 
direction.

- The amount of light absorbed by the LTV sensor from a given direction would be proportional to the area of the LTV sensor lens as seen from that direction.

With these assumptions, the output of the LTV sensor varies with the relative position of the LED with the respect to the LTV sensor through three parameters: the distance between the centres of the LED and LTV sensor $(r)$, the angle between the normal of the LED and the line connecting the centres of the LED and LTV sensor $(\theta)$, and the angle between the normal of the LTV sensor and the line connecting the centres of the LED and LTV sensor $(\phi)$. The three parameters are highlighted in Fig. 4.8. In addition to the above assumptions, the derivation of the proposed model for the LTV sensor voltage relies on the proportionality between the irradiance absorbed by the LTV sensor and the output voltage [97].

Given the assumption that the light from the LED diffuses along a sphere, the LTV voltage would decrease with the inverse square of $r$. For the current sensor, the voltage of the LED was assumed to be proportional to the general inverse quadratic relationship,

$$
V_{L T V} \propto \frac{A_{1}}{B_{1} r^{2}+C_{1} r+D_{1}}
$$

where $A_{i}, B_{i}, C_{i}$, and $D_{i}$ are constants to be determined. With the assumptions that the light emitted by the LED to the LTV sensor and the light absorbed by the LTV sensor is proportional to the area viewed by the other component, the 


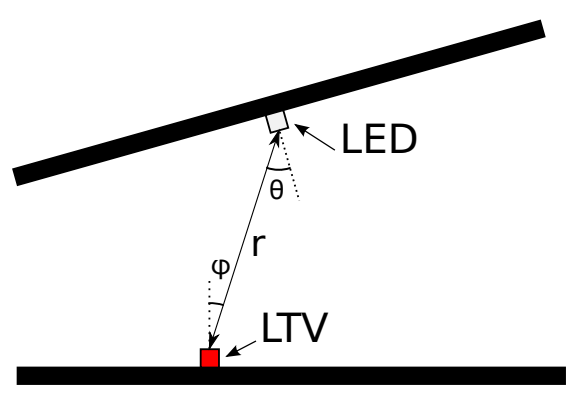

Figure 4.8: The parameters of the relative position between the LED and LTV sensor affecting the LTV output voltage.

effects of $\theta$ and $\phi$ on the LTV sensor output voltage are approximated as,

$$
\begin{aligned}
& V_{L T V} \propto A_{2} \cos \left(B_{2} \theta\right), \\
& V_{L T V} \propto A_{3}\left(1+B_{3} \cos \left(C_{3} \phi\right)\right) .
\end{aligned}
$$

The above approximations for the effects of $r, \theta$ and $\phi$ are independent and will each be proportional to the LTV sensor output voltage. To combine the effects of (4.9)-(4.11), the equations are multiplied together, allowing LTV sensor voltage to be determined from,

$$
V_{L T V}=\frac{V_{0}}{r^{2}+A r+B} \cos (C \theta)(1+D \cos (E \phi))
$$

where $V_{0}, A, B, C, D$ and $E$ are constants to be determined.

\subsubsection{Initial LED and LTV sensor calibration}

To test the model and determine initial constants for (4.12), each LED and LTV sensor was calibrated by varying one parameter $(r, \theta$ and $\phi)$ while keeping the 


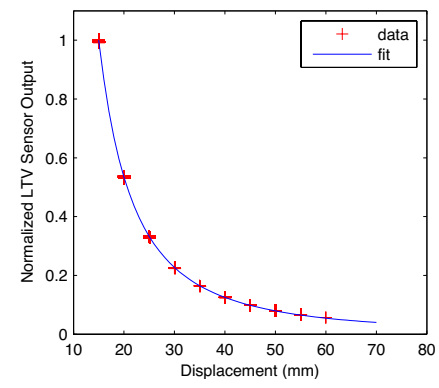

(a)

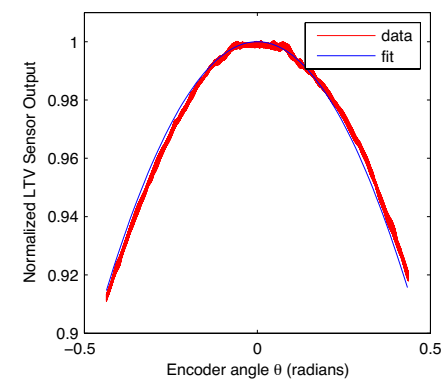

(b)

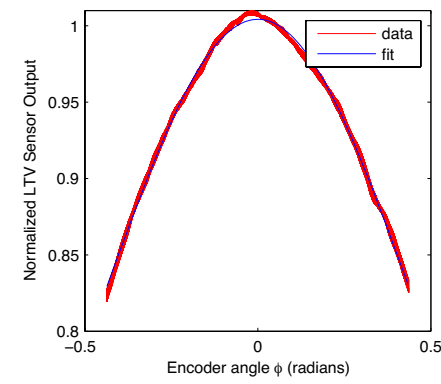

(c)

Figure 4.9: Sample curve fits for the effect of a) $r$, b) $\theta$ and c) $\phi$ on the LTV sensor output.

other parameters constant. The calibration procedure was performed using a ball screw actuator to supply the linear displacements to calibrate the effect of $r$, while a motor with attached encoder rotated the LED and LTV sensor to provide the angular displacements to calibrate the effects of $\theta$ and $\phi$. The Matlab curve fitting toolbox was then used to determine the parameters of (4.9)-(4.11) for each LED and LTV sensor. Sample fits can be seen in Fig. 4.9. From Fig. 4.9, it can be seen that the current model predicts the general trends in the data; however, deviations exist. These deviations stem from multiple sources of error including sensor noise due to the actuation of the calibration rig, imperfections on the LTV sensor lens, and deviations in the mounting of the LED/LTV sensor from the center of the calibration axis of rotation. It should also be noted that the assumptions used in the model will fail with large angles $\theta$ and $\phi$, and small distances $r$.

\subsubsection{CLS calibration}

Once assembled, the CLS was calibrated to determine the positions and orientations of all LEDs and LTV sensors, in addition to the values required for (4.12) 
for each LTV sensor and LED. To calibrate the CLS, the CLS was subjected to general motion by hand while the Optotrak system measured the external shell at a rate of $80 \mathrm{~Hz}$ while a QPID DAQ measured the LTV sensor voltages at a rate of $2 \mathrm{kHz}$. The Optotrak was externally triggered to allow synchronization of the measurements.

The Levenberg-Marquardt algorithm was then employed to determine the required LED and LTV sensor positions, orientations and constants to minimize the sum of squared differences between the measured and expected voltages across all data points for the calibration trial. The cost function was adapted by adding a term to force the LED and LTV sensors to be oriented toward one another. In the batch calibration, LTV sensors with voltage measurements greater than $0.8 \mathrm{~V}$, $r<20 \mathrm{~mm}$ or $|\theta|<40^{\circ}$ were removed from the calibration. Values determined in the initial calibration and measurements taken from the prototype were used as initial values for the calibration.

\subsubsection{Pose Estimation and Performance}

With the calibrated values, the Levenberg-Marquardt algorithm was employed to estimate the external shell pose by determining the pose to minimize the squared difference between the measured and calculated LTV sensor voltages for the given estimated pose. A weighting function was incorporated to decrease the weight of an LTV sensor if $15 \mathrm{~mm}<r<60 \mathrm{~mm}$ or $-45^{\circ}<\theta<45^{\circ}$. The initial value for the numerical method was the previously determined pose. To test the performance of the optical CLS, the pose estimation was employed with measured LTV sensor voltages from five CLS trajectories; general motion (the calibration 
Table 4.5: Statistics summarizing the accuracy of the optical CLS to predict motion in the plane of interest for given ranges of deformation $D$

\begin{tabular}{|l|l|l|l|l|}
\cline { 3 - 5 } \multicolumn{2}{l|}{} & $\begin{array}{l}\text { Magnitude of } \\
\text { compression error }\end{array}$ & $\begin{array}{l}\text { Magnitude of } \\
\text { shear error }\end{array}$ & $\begin{array}{l}\text { Magnitude of } \\
\text { rotation error }\end{array}$ \\
\hline \multirow{2}{*}{$\mathrm{D}<5 \mathrm{~mm}$} & $\bar{e}$ & $0.7905 \mathrm{~mm}$ & $0.9285 \mathrm{~mm}$ & $0.1883^{\circ}$ \\
\cline { 2 - 5 } & $s_{e}$ & $2.0494 \mathrm{~mm}$ & $2.8254 \mathrm{~mm}$ & $0.2357^{\circ}$ \\
\hline \multirow{2}{*}{$5 \mathrm{~mm} \leq \mathrm{D}<10 \mathrm{~mm}$} & $\overline{x_{e}}$ & $1.9181 \mathrm{~mm}$ & $0.7991 \mathrm{~mm}$ & $0.485^{\circ}$ \\
\cline { 2 - 6 } & $s_{e}$ & $2.4114 \mathrm{~mm}$ & $1.2745 \mathrm{~mm}$ & $0.5825^{\circ}$ \\
\hline \multirow{2}{*}{$10 \mathrm{~mm} \leq \mathrm{D}<15 \mathrm{~mm}$} & $\bar{e}$ & $1.6571 \mathrm{~mm}$ & $0.7514 \mathrm{~mm}$ & $0.3413^{\circ}$ \\
\cline { 2 - 6 } & $s_{e}$ & $1.5281 \mathrm{~mm}$ & $0.7838 \mathrm{~mm}$ & $0.4712^{\circ}$ \\
\hline \multirow{2}{*}{$\mathrm{D} \geq 20 \mathrm{~mm}$} & $\bar{e}$ & $2.846 \mathrm{~mm}$ & $0.5003 \mathrm{~mm}$ & $1.0987^{\circ}$ \\
\cline { 2 - 6 } & $s_{e}$ & $1.3311 \mathrm{~mm}$ & $0.5185 \mathrm{~mm}$ & $0.4455^{\circ}$ \\
\hline
\end{tabular}

trajectory), compression, shear, rotation and rest. Summarizing statistics of the sensor performance can be found in Table 4.5. Plots of the predicted and measured compression, shear and rotational motion can be seen in Fig. 4.10. The numerical method when coded in C using C MINPACK [98] had an average computation time of $<0.1 \mathrm{~ms}$.

\subsubsection{Discussion}

From Fig. 4.10, it can be seen that the optical based CLS is able to follow the general trends in the motion; however, significant deviation from the measured motion is observed. This is noted in the mean magnitudes of error seen in Table 4.5. In addition, the standard deviations in the error are large. This is of greater concern-a large spread in the errors makes the sensor less reliable. 

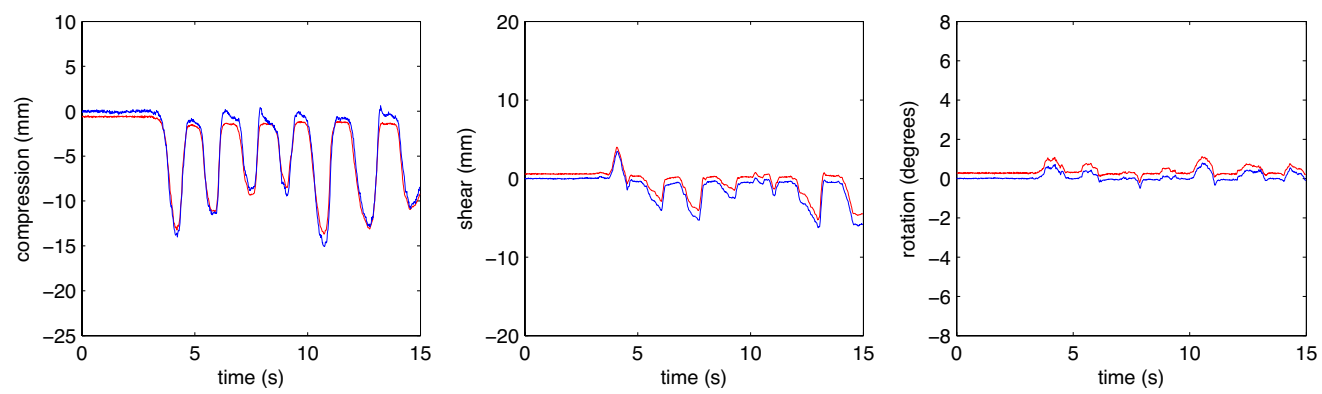

(a) Compression Trial
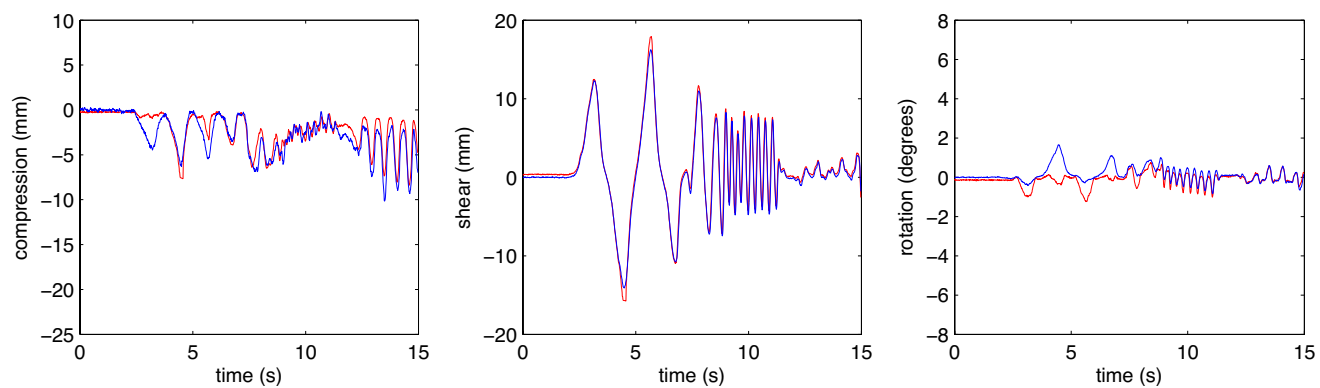

(b) Shear Trial
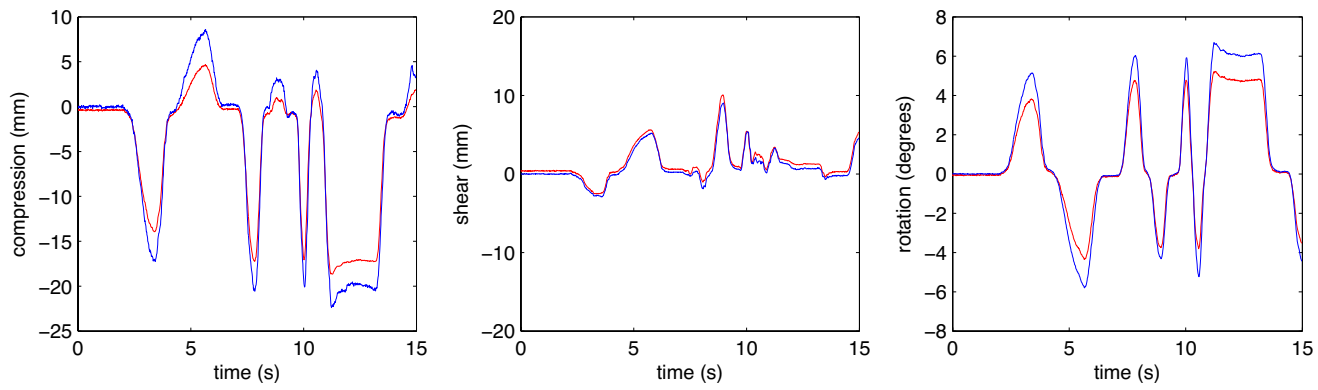

(c) Rotation Trial

Figure 4.10: Measured (blue) and estimated (red) motion and orientations in the $\mathrm{x}-\mathrm{z}$ plane. 
The errors in pose estimation for the optical based sensor are believed to stem from errors in the LTV sensor modelling and from out of plane motion in the sensor. The LTV sensor model does not fit the measured voltages exactly (see Fig. 4.9) and the model will fail for small $r$ and large $\theta$ and $\phi$. The brackets and slots were meant to eliminate out of plane motion; however, out of plane motions of up to $2.515 \mathrm{~mm}$ and $7.34^{\circ}$ were still observed. As was the case with the potentiometer based solution, such out of plane motion would not be captured by the current sensor model as all LTV sensors and LEDs are located in a plane. For the potentiometer based solution, out of plane motion resulted in changes of the orientations and offsets of the joint axes resulting in errors; out of plane motion in the optical sensor causes errors due to changes of LTV sensor outputs. As the LTV sensor outputs are combined through a series of nonlinear equations, the errors due to voltage changes are not limited by the physical out of plane motion as was the case with the potentiometer based sensor. The changes of LTV sensor voltages can result in significantly different estimates of pose as the numerical algorithm seeks a pose in the plane that matches the voltage measurements caused by a shell pose outside of the plane. The out of plane motion also existed in the calibration data and would have influenced the selection of the constants and locations for each LED and LTV sensor, affecting all estimates. The significant effect of out of plane motion and the LTV sensor modelling errors are believed to have caused the optical sensor to be less accurate than the potentiometer based solution. Further prototypes need to either have better mechanical constraints, or should be designed for 6 DoF motion to eliminate the need for even simple kinematic constraints. The current equations to model the planar shell can already be employed for the $6 \mathrm{DoF}$ case. 
The current optical based CLS cannot be used for the proposed SOIS control methodology. The magnitude and spread of the errors in pose estimation would affect the behaviour of the proposed controller by commanding large undesired motions. From Fig. 4.10, it is also seen that the sensor is insensitive to some motion and either does not estimate the full magnitude of deformation or does not register deformation at all. In the SOIS, this would result in slow response times, and larger deformations, $\vec{x}_{d}$, and contact forces. However, the ability of the current sensor to track the general motion of the external shell shows potential for the sensing methodology. With further development, the optical sensor based technology could be used for the proposed safety measure on the ViGRR platform.

\subsection{Summary}

A potentiometer based CLS and an optical sensor based CLS were developed as an initial effort towards implementing the SOIS on the ViGRR. The potentiometer based sensor was prototyped to provide a test platform for initial testing of the SOIS control law and to experimentally optimize the CLS compliance. To this end, the potentiometer based sensor was designed for accurate and simple pose estimation while allowing varying stiffness and damping across the joints. Experiments evaluated the accuracy of the potentiometer based sensor to estimate external shell pose and it was determined the sensor was suitable for initial testing. The optical based sensor was developed to test the ability of optical sensors to estimate the external shell pose without the need of a kinematic chain. The sensor was found to have large errors in pose estimation and the current sensor was not suitable for implementing the SOIS control law. However, the ability 
of the sensor to track general trends in the motion illustrated the potential for optical based sensing in a future final CLS design. 


\section{Chapter 5}

\section{Conclusions and Future Work}

As robots continue to expand into new applications involving HRI, the need for safety measures to prevent user injury grows. While many safety measures exist in the literature, the most mature technology requires the use of light weight design and compliant, instrumented joints. Existing robots with higher inertia require new methods to ensure user safety during unexpected collisions. The use of compliant tactile sensors to detect and respond to collision presents the opportunity to ensure safety for such robots. However, the current tactile sensor technologies are limited by the use of sensor arrays, the inability to detect shear forces and the lack of an existing control strategy to ensure stable interaction post collision.

The safety oriented interaction strategy (SOIS) was proposed to prevent user injury during interaction with the existing high performance Virtual Gait Rehabilitation Robot. By employing the novel compliant limb sensor (CLS) and a deformation feedback force control law, the SOIS decouples the link inertia from the collision and prevents the subsequent build up of forces and the complete compression of the CLS. By preventing the build up for forces, the SOIS decreases 
the risk of injury as determined by the existing literature in robot safety. The objective of the thesis was to serve as a initial development of the SOIS. This goal was met by the development of the force control strategy and prototyping and testing of two compliant limb sensor designs.

\subsection{Conclusions}

The current thesis included control theory development, analytical evaluation of controller performance, simulation and implementation of a zero force control law, as well as sensor development. The completed work performed throughout the thesis is summarized below:

\section{Analytical analysis of collision response control law}

A zero force control law using the measured deformation provided by the CLS was selected considering the existing force control laws and the current application. The control law was then analyzed analytically. Unlike previous analyses, the current work includes friction in the sensor and is not limited to proving stability. For a 1DoF linear time invariant system and CLS, conditions for the stability of the control law when interacting with passive environments was developed. The ability of the controller to prevent complete compression of the CLS was evaluated and determined to be proportional to the $K_{d}$ gain employed. Increasing the $K_{p}$ control gain was found to decrease the low frequency perceived impedance while the $K_{d}$ gain decreased the perceived impedance at slightly higher frequencies. The high frequency robot impedance could not be influenced by the control law and was dictated by the CLS inertia. 


\section{Evaluation of control law through experiments and simula- tion}

A design method to implement the proposed zero force control law was developed and employed to design a control law for a 1 DoF test platform. The procedure was developed considering many aspects of safety, differentiating it from a standard force control law design procedure. The control law was simulated for the linear model of the test platform and 1DoF CLS to investigate the effect of force saturation, sampling frequency, sensor noise, and stiction. The effect of these nonlinearities on controller behavior was not previously explored for deformation feedback. Force saturation was found to not significantly affect the low speed interaction but decreased the response time of the system to impact. Sampling frequency was found to increase oscillations and instability when decreased to $200 \mathrm{~Hz}$ and sensor noise was found to add oscillations in the response as the controller attempted to follow the noise signal. Stiction produced steady state velocities when interacting with an inertia and a limit cycle when interacting with environments incorporating a stiffness. A threshold on the CLS deformation fed back into the control law prevented these behaviours. The control law was then implemented on the test platform and compared to an admittance control law during impact trials and while interacting with varying environments. The proposed controller was shown to be stable, while displaying similar behaviour as the admittance control law. 


\section{Development of potentiometer based planar CLS}

A potentiometer based CLS to facilitate ViGRR experiments was developed employing a 3 link open kinematic chain to allow measurement of planar motion using 1 DoF potentiometers. The kinematics of the CLS were calibrated using motion capture data. The known kinematics and the measured joint deflections allowed accurate estimation of motion within the plane, though the inertia of the kinematic chain was large. While the sensing methods and sensor design are not novel, the potentiometer based CLS contributes a valuable test platform to the future development of the SOIS and the advancement of the ViGRR project.

\section{Development of optical based planar CLS}

In collaboration with other ABL researchers, a planar optical sensor based CLS was developed to determine the feasibility of optical sensors to determine deformation in the final sensor design. The optical based planar CLS employs a novel, low cost sensing methodology consisting of LTV sensors and LEDs to estimate the pose of the external shell. The CLS could follow the general shape of the external shell motion, but suffered from low accuracy with high spread in errors. With further development, the prototype demonstrates the possibility for use in the final design.

\subsection{Future Work}

With the outline of the SOIS completed, future work will focus on the extension and refinement of the presented work to allow full scale implementation on the 
ViGRR platform. To reach this goal, specific future research tasks include:

\section{Refinement of the optical based Compliant Limb Sensor}

To be employed as the final CLS design for the SOIS, the pose estimation of the optical based sensor must be refined and the compliance of the sensor must be optimized for use with ViGRR. To improve the accuracy and spread of error for the optical based sensor, it is proposed to redesign the CLS to estimate pose in 6 DoF, such that kinematic restrictions are not required and out of plane motion is accounted for in pose estimation. The $6 \mathrm{DoF}$ design would require an optimal placement of LED and LTV sensors to be determined. The cost function of the pose estimation could also be modified to improve estimation accuracy. The optimized compliance of the final sensor should be determined experimentally through impact testing with soft tissue using the potentiometer based CLS. The optimization should be performed experimentally, as simulations will not capture the nonlinear dynamics of the soft tissue or the unmodelled dynamics of the ViGRR, such as the harmonic drive torque ripple and compliance.

\section{Extension of the proposed controller to the nonlinear, multi degree of freedom, rehabilitation case}

To be implemented on the ViGRR, the proposed control law must be extended to the planar case with nonlinear CLS and robot dynamics. One method to extend to the multi degree of freedom case is the use of feedback linearization to shape the ViGRR's dynamics at the CLS into a linear decoupled system. If the CLS dynamics are then designed to approach linear behaviour for small deformations, 
the above control theory could be applied. Such an approach would require an analysis of the sensitivity of the controller to unmodelled dynamics in the feedback linearization and experimentation on the ViGRR to ensure the robot is behaving

appropriately. In extending to the multi degree of freedom case, the projection of the commands into the null space of the ViGRR must also be investigated as the current 1DoF system model does not contain a null space with which to perform the analysis.

\section{Development of strategies to switch control laws during collision}

In case of unexpected impacts, the control law must be switched from the current task to the force control law. As previously discussed, the ViGRR has a redundant degree of freedom that allows for collision response without the need to stop the current task and the position commands of the proposed controller are compatible with the ViGRR admittance control law. A strategy must be developed to allow minor collisions to be mitigated through the use of the redundant joint while larger collisions suspend the task. A post collision strategy must also be developed to determine if the ViGRR should return to the task after collision or if the system must be reset and therapy restarted. 


\section{List of References}

[1] S. Haddadin, A. Albu-Schäffer, and G. Hirzinger, "Requirements for safe robots: Measurements, analysis and new insights," The International Journal of Robotics Research, vol. 28, no. 11-12, pp. 1507-1527, 2009.

[2] S. Yigit, C. Burghart, and H. Woern, "Applying reflexes to enhance safe human-robot-co-operation with a humanlike robot arm," in Proc., 35th International Symposium on Robotics, 2004.

[3] K. Chisholm, K. Klumper, A. Mullins, and M. Ahmadi, "A task oriented haptic gait rehabilitation robot," Mechatronics, vol. 24, no. 8, pp. 1083-1091, 2014.

[4] I. F. of Robotics Statistical Department, "Executive summary of world robotics 2014." http://www.worldrobotics.org/uploads/media/ Executive_Summary_WR_2014_02.pdf, 2014.

[5] U. Hillenbrand, C. Ott, B. Brunner, C. Borst, and G. Hirzinger, "Towards service robots for the human environment: the robutler," Mechatronics and Robotics (MECHROB), pp. 1497-1502, 2004.

[6] B. Tribelhorn and Z. Dodds, "Evaluating the roomba: A low-cost, ubiquitous platform for robotics research and education," in Robotics and Automation, 2007 IEEE International Conference on, pp. 1393-1399, IEEE, 2007.

[7] G. Song, K. Yin, Y. Zhou, and X. Cheng, "A surveillance robot with hopping capabilities for home security," Consumer Electronics, IEEE Transactions on, vol. 55, no. 4, pp. 2034-2039, 2009. 
[8] K. Kosuge, T. Hayashi, Y. Hirata, and R. Tobiyama, "Dance partner robotms dancer," in Intelligent Robots and Systems, 2003.(IROS 2003). Proceedings. 2003 IEEE/RSJ International Conference on, vol. 4, pp. 3459-3464, IEEE, 2003.

[9] M. Pietrusinski, I. Cajigas, G. Severini, P. Bonato, and C. Mavroidis, "Robotic gait rehabilitation trainer," Mechatronics, IEEE/ASME Transactions on, vol. 19, no. 2, pp. 490-499, 2014.

[10] A. Morbi, M. Ahmadi, and A. Nativ, "Gaitenable: An omnidirectional robotic system for gait rehabilitation," in Mechatronics and Automation (ICMA), 2012 International Conference on, pp. 936-941, IEEE, 2012.

[11] M. T. Gettman, M. L. Blute, G. K. Chow, R. Neururer, G. Bartsch, and R. Peschel, "Robotic-assisted laparoscopic partial nephrectomy: technique and initial clinical experience with davinci robotic system," Urology, vol. 64, no. 5, pp. 914-918, 2004.

[12] C. D. Kidd, W. Taggart, and S. Turkle, "A sociable robot to encourage social interaction among the elderly," in Robotics and Automation, 2006. ICRA 2006. Proceedings 2006 IEEE International Conference on, pp. 3972-3976, IEEE, 2006.

[13] S. Y. Okita, V. Ng-Thow-Hing, and R. Sarvadevabhatla, "Learning together: Asimo developing an interactive learning partnership with children," in Robot and Human Interactive Communication, 2009. RO-MAN 2009. The 18th IEEE International Symposium on, pp. 1125-1130, IEEE, 2009.

[14] J. Heinzmann and A. Zelinsky, "Quantitative safety guarantees for physical human-robot interaction," The International Journal of Robotics Research, vol. 22, no. 7-8, pp. 479-504, 2003.

[15] M. Nokata and N. Tejima, "10 a safety strategy for rehabilitation robots," in Advances in Rehabilitation Robotics, pp. 177-185, Springer, 2004.

[16] K. Ikuta and M. Nokata, "General evaluation method of safety for humancare robots," in Robotics and Automation, 1999. Proceedings. 1999 IEEE International Conference on, vol. 3, pp. 2065-2072, IEEE, 1999. 
[17] S. Wang, J. Ding, J. Yun, Q. Li, and B. Han, "A robotic system with force feedback for micro-surgery," in Robotics and Automation, 2005. ICRA 2005. Proceedings of the 2005 IEEE International Conference on, pp. 199-204, IEEE, 2005.

[18] B. Povse, D. Koritnik, R. Kamnik, T. Bajd, and M. Munih, "Emulation system for assessment of human-robot collision," Meccanica, vol. 46, no. 6, pp. 1363-1371, 2011.

[19] G. Rosati, "The place of robotics in post-stroke rehabilitation," Expert Review of Medical Devices, vol. 7, no. 6, pp. 753-758, 2010.

[20] R. R. Murphy, "Rescue robotics for homeland security," Communications of the ACM, vol. 47, no. 3, pp. 66-68, 2004.

[21] M. Zinn, O. Khatib, B. Roth, and J. K. Salisbury, "Playing it safe [humanfriendly robots]," Robotics $\&$ Automation Magazine, IEEE, vol. 11, no. 2, pp. 12-21, 2004.

[22] G. Tonietti, R. Schiavi, and A. Bicchi, "Design and control of a variable stiffness actuator for safe and fast physical human/robot interaction," in Robotics and Automation, 2005. ICRA 2005. Proceedings of the 2005 IEEE International Conference on, pp. 526-531, IEEE, 2005.

[23] D. Gao and C. W. Wampler, "Head injury criterion," Robotics \& Automation Magazine, IEEE, vol. 16, no. 4, pp. 71-74, 2009.

[24] S. Haddadin, A. Albu-Schäffer, and G. Hirzinger, "Safety evaluation of physical human-robot interaction via crash-testing.," in Robotics: Science and Systems, vol. 3, pp. 217-224, 2007.

[25] T. S. Tadele, T. de Vries, and S. Stramigioli, "The safety of domestic robotics: A survey of various safety-related publications," Robotics \& A Automation Magazine, IEEE, vol. 21, no. 3, pp. 134-142, 2014.

[26] Y. Yamada, Y. Hirasawa, S. Huang, and Y. Umetani, "Fail-safe human/robot contact in the safety space," in Robot and Human Communication, 1996., 5th IEEE International Workshop on, pp. 59-64, IEEE, 1996. 
[27] B. Povse, D. Koritnik, T. Bajd, and M. Munih, "Correlation between impactenergy density and pain intensity during robot-man collision," in Biomedical Robotics and Biomechatronics (BioRob), 2010 3rd IEEE RAS and EMBS International Conference on, pp. 179-183, IEEE, 2010.

[28] B. Povse, D. Koritnik, R. Kamnik, T. Bajd, and M. Munih, "Industrial robot and human operator collision," in Systems Man and Cybernetics (SMC), 2010 IEEE International Conference on, pp. 2663-2668, IEEE, 2010.

[29] B. Povse, D. Koritnik, T. Bajd, and M. Munih, "Mechanical model of human lower arm," in Proceedings of the seventh annual ACM/IEEE international conference on Human-Robot Interaction, pp. 209-210, ACM, 2012.

[30] J.-J. Park, S. Haddadin, J.-B. Song, and A. Albu-Schäffer, "Designing optimally safe robot surface properties for minimizing the stress characteristics of human-robot collisions," in Robotics and Automation (ICRA), 2011 IEEE International Conference on, pp. 5413-5420, IEEE, 2011.

[31] M. Wassink and S. Stramigioli, "Towards a novel safety norm for domestic robotics," in Intelligent Robots and Systems, 200\%. IROS 200\%. IEEE/RSJ International Conference on, pp. 3354-3359, IEEE, 2007.

[32] S. Haddadin, A. Albu-Schaffer, and G. Hirzinger, "The role of the robot mass and velocity in physical human-robot interaction-part i: Non-constrained blunt impacts," in Robotics and Automation, 2008. ICRA 2008. IEEE International Conference on, pp. 1331-1338, IEEE, 2008.

[33] S. Haddadin, A. Albu-Schaffer, M. Frommberger, and G. Hirzinger, "The role of the robot mass and velocity in physical human-robot interaction-part ii: Constrained blunt impacts," in 2008 IEEE International Conference on Robotics and Automation.

[34] S. Haddadin, A. Albu-Schäffer, and G. Hirzinger, "Soft-tissue injury in robotics," in Robotics and Automation (ICRA), 2010 IEEE International Conference on, pp. 3426-3433, IEEE, 2010.

[35] S. Haddadin, S. Haddadin, A. Khoury, T. Rokahr, S. Parusel, R. Burgkart, A. Bicchi, and A. Albu-Schäffer, "On making robots understand safety: 
Embedding injury knowledge into control," The International Journal of Robotics Research, vol. 31, no. 13, pp. 1578-1602, 2012.

[36] R. Hirschfeld, F. Aghazadeh, and R. Chapleski, "Survey of robot safety in industry," International Journal of Human Factors in Manufacturing, vol. 3, no. 4, pp. 369-379, 1993.

[37] H. M. Parsons, "Human factors in industrial robot safety," Journal of occupational accidents, vol. 8, no. 1, pp. 25-47, 1986.

[38] B. Lacevic, P. Rocco, and A. M. Zanchettin, "Safety assessment and control of robotic manipulators using danger field," Robotics, IEEE Transactions on, vol. 29, no. 5, pp. 1257-1270, 2013.

[39] G. Buizza Avanzini, N. M. Ceriani, A. M. Zanchettin, P. Rocco, and L. Bascetta, "Safety control of industrial robots based on a distributed distance sensor," Control Systems Technology, IEEE Transactions on, vol. 22, no. 6, pp. 2127-2140, 2014.

[40] F. Flacco, T. Kröger, A. De Luca, and O. Khatib, "A depth space approach to human-robot collision avoidance," in Robotics and Automation (ICRA), 2012 IEEE International Conference on, pp. 338-345, IEEE, 2012.

[41] A. Pervez and J. Ryu, "Safe physical human robot interaction-past, present and future," Journal of Mechanical Science and Technology, vol. 22, no. 3, pp. 469-483, 2008.

[42] H.-o. Lim and K. Tanie, "Collision-tolerant control of human-friendly robot with viscoelastic trunk," Mechatronics, IEEE/ASME Transactions on, vol. 4, no. 4, pp. 417-427, 1999.

[43] H.-o. Lim, M. Sunagawa, and N. Takeuchi, "Development of human-friendly robot with collision force suppression mechanism," in ICCAS-SICE, 2009, pp. 5712-5716, IEEE, 2009.

[44] K. Shimamoto, N. Takeuchi, and H.-o. Lim, "Development of collision force suppression mechanism for human-friendly robot," in Control, Automation and Systems (ICCAS), 2011 11th International Conference on, pp. 665-670, IEEE, 2011. 
[45] N. Tejima and D. Stefanov, "Fail-safe components for rehabilitation robots-a reflex mechanism and fail-safe force sensor," in Rehabilitation Robotics, 2005. ICORR 2005. 9th International Conference on, pp. 456-460, IEEE, 2005.

[46] S.-S. Yoon, S. Kang, S.-k. Yun, S.-J. Kim, Y.-H. Kim, and M. Kim, "Safe arm design with mr-based passive compliant joints and viscoelastic covering for service robot applications," Journal of mechanical science and technology, vol. 19, no. 10, pp. 1835-1845, 2005.

[47] Y. Yamada, Y. Hirasawa, S. Huang, Y. Umetani, and K. Suita, "Humanrobot contact in the safeguarding space," Mechatronics, IEEE/ASME Transactions on, vol. 2, no. 4, pp. 230-236, 1997.

[48] S. Haddadin, A. Albu-Schäffer, A. D. Luca, and G. Hirzinger, "Collision detection and reaction: A contribution to safe physical human-robot interaction," in Intelligent Robots and Systems, 2008. IROS 2008. IEEE/RSJ International Conference on, pp. 3356-3363, IEEE, 2008.

[49] T. S. Dahl and A. Paraschos, "A force-distance model of humanoid arm withdrawal reflexes," in Advances in Autonomous Robotics, pp. 13-24, Springer, 2012.

[50] T. S. Dahl and A. Palmer, "Touch-triggered protective reflexes for safer robots," in Proceedings of the International Symposium on New Frontiers in Human-Robot Interaction, pp. 27-33, 2010.

[51] S. Chiaverini and L. Sciavicco, "The parallel approach to force/position control of robotic manipulators," Robotics and Automation, IEEE Transactions on, vol. 9, no. 4, pp. 361-373, 1993.

[52] G. Zeng and A. Hemami, "An overview of robot force control," Robotica, vol. 15, no. 05, pp. 473-482, 1997.

[53] O. Khatib, B. Siciliano, and S. O. service), Springer handbook of robotics. Berlin: Springer, 2008.

[54] N. Hogan, "Stable execution of contact tasks using impedance control," in Robotics and Automation. Proceedings. 1987 IEEE International Conference on, vol. 4, pp. 1047-1054, IEEE, 1987. 
[55] N. Hogan, "Impedance control: An approach to manipulation," in American Control Conference, 1984, pp. 304-313, IEEE, 1984.

[56] N. Hogan, "Impedance control: An approach to manipulation: Part itheory," Journal of dynamic systems, measurement, and control, vol. 107, no. 1, pp. 1$7,1985$.

[57] N. Hogan, "Impedance control: An approach to manipulation: Part iiimplementation," Journal of dynamic systems, measurement, and control, vol. 107, no. 1, pp. 8-16, 1985.

[58] N. Hogan, "Impedance control: An approach to manipulation: Part iiiapplications," Journal of dynamic systems, measurement, and control, vol. 107, no. 1, pp. 17-24, 1985 .

[59] R. J. Adams and B. Hannaford, "Control law design for haptic interfaces to virtual reality," IEEE Transactions on Control Systems Technology, vol. 10, no. 1, pp. 3-13, 2002.

[60] D. A. Lawrence, "Impedance control stability properties in common implementations," in Robotics and Automation, 1988. Proceedings., 1988 IEEE International Conference on, pp. 1185-1190, IEEE, 1988.

[61] T. Tsuji, A. Jazidie, and M. Kaneko, "Multi-point impedance control for redundant manipulators," Systems, Man, and Cybernetics, Part B: Cybernetics, IEEE Transactions on, vol. 26, no. 5, pp. 707-718, 1996.

[62] R. J. Anderson and M. W. Spong, "Hybrid impedance control of robotic manipulators," Robotics and Automation, IEEE Journal of, vol. 4, no. 5, pp. 549-556, 1988.

[63] C. Ott, R. Mukherjee, and Y. Nakamura, "Unified impedance and admittance control," in Robotics and Automation (ICRA), 2010 IEEE International Conference on, pp. 554-561, IEEE, 2010.

[64] D. E. Whitney, "Force feedback control of manipulator fine motions," Journal of Dynamic Systems, Measurement, and Control, vol. 99, no. 2, pp. 91-97, 1977. 
[65] R. Q. Van der Linde, P. Lammertse, E. Frederiksen, and B. Ruiter, "The hapticmaster, a new high-performance haptic interface," in Proc. Eurohaptics, pp. 1-5, 2002.

[66] K. J. Chisholm, C. U. Theses, M. Dissertations. Engineering, and P. (Firm), "Design and control for a gait rehabilitation robot," 2010.

[67] L. L. Kovács, G. Stépán, and T. Insperger, "Outer-loop force control of industrial robots," in Proceedings of the 11th World Congress in Mechanism and Machine Science, China Machinery Press, Tianjin, China, pp. 17461750, 2004.

[68] L. L. Kovács and G. Stépán, "Dynamics of digital force control applied in rehabilitation robotics," Meccanica, vol. 38, no. 2, pp. 213-226, 2003.

[69] F. Lange, C. Jehle, M. Suppa, and G. Hirzinger, "Revised force control using a compliant sensor with a position controlled robot," in 2012 IEEE International Conference on Robotics and Automation (ICRA), pp. 15321537, IEEE, 2012.

[70] F. Lange, W. Bertleff, and M. Suppa, "Force and trajectory control of industrial robots in stiff contact," in 2013 IEEE International Conference on Robotics and Automation (ICRA), pp. 2927-2934, IEEE, 2013.

[71] F. Lange, "Method for force or moment control of robots, involves providing task with target movement of robot and target forces or target moments, which are practiced by tool on work piece," Mar. 17 2011. DE Patent App. DE200,910,040,194.

[72] F. Lange, M. Suppa, and G. Hirzinger, "Control with a compliant forcetorque sensor," in Proceedings of ROBOTIK 2012; 7th German Conference on Robotics, pp. 1-6, VDE, 2012.

[73] R. S. Dahiya, G. Metta, M. Valle, and G. Sandini, "Tactile sensingfrom humans to humanoids," Robotics, IEEE Transactions on, vol. 26, no. 1, pp. 120,2010 .

[74] P. S. Girão, P. M. P. Ramos, O. Postolache, and J. M. D. Pereira, "Tactile sensors for robotic applications," Measurement, vol. 46, no. 3, pp. 1257-1271, 2013. 
[75] M. Battaglia, L. Blanchet, A. Kheddar, S. Kajita, and K. Yokoi, "Combining haptic sensing with safe interaction," in Intelligent Robots and Systems, 2009. IROS 2009. IEEE/RSJ International Conference on, pp. 231-236, IEEE, 2009.

[76] V. Duchaine, N. Lauzier, M. Baril, M.-A. Lacasse, and C. Gosselin, "A flexible robot skin for safe physical human robot interaction," in Robotics and Automation, 2009. ICRA'09. IEEE International Conference on, pp. 36763681, IEEE, 2009.

[77] M. Strohmayr and D. Schneider, "The dlr artificial skin step ii: Scalability as a prerequisite for whole-body covers," in Intelligent Robots and Systems (IROS), 2013 IEEE/RSJ International Conference on, pp. 4721-4728, Nov 2013.

[78] M. Strohmayr, H. Worn, and G. Hirzinger, "The dlr artificial skin step i: Uniting sensitivity and collision tolerance," in Robotics and Automation (ICRA), 2013 IEEE International Conference on, pp. 1012-1018, IEEE, 2013.

[79] M. Frigola, A. Casals, and J. Amat, "Human-robot interaction based on a sensitive bumper skin," in Intelligent Robots and Systems, 2006 IEEE/RSJ International Conference on, pp. 283-287, IEEE, 2006.

[80] J. Ulmen and M. Cutkosky, "A robust, low-cost and low-noise artificial skin for human-friendly robots," in Robotics and Automation (ICRA), 2010 IEEE International Conference on, pp. 4836-4841, IEEE, 2010.

[81] K. Klumper, "Haptic foot interactions on a 4-link planar rehabilitation robot," 2012. Copyright - Copyright ProQuest, UMI Dissertations Publishing 2012; Last updated - 2015-07-08; First page - n/a.

[82] N. Hogan, "Controlling impedance at the man/machine interface," in Robotics and Automation, 1989. Proceedings., 1989 IEEE International Conference on, pp. 1626-1631, IEEE, 1989.

[83] W. S. Newman, "Stability and performance limits of interaction controllers," Journal of dynamic systems, measurement, and control, vol. 114, no. 4, pp. 563-570, 1992. 
[84] S. P. Buerger and N. Hogan, "Complementary stability and loop shaping for improved human-robot interaction," IEEE Transactions on Robotics, vol. 23, no. 2, pp. 232-244, 2007.

[85] R. J. Adams and B. Hannaford, "Stable haptic interaction with virtual environments," IEEE Transactions on Robotics and Automation, vol. 15, no. 3, pp. 465-474, 1999.

[86] A. Morbi, M. Ahmadi, A. D. Chan, and R. Langlois, "Stability-guaranteed assist-as-needed controller for powered orthoses," Control Systems Technology, IEEE Transactions on, vol. 22, no. 2, pp. 745-752, 2014.

[87] L. M. Miller and J. Rosen, "Comparison of multi-sensor admittance control in joint space and task space for a seven degree of freedom upper limb exoskeleton," in Biomedical Robotics and Biomechatronics (BioRob), 2010 3rd IEEE RAS and EMBS International Conference on, pp. 70-75, IEEE, 2010.

[88] J. E. Colgate and N. Hogan, "Robust control of dynamically interacting systems," International journal of Control, vol. 48, no. 1, pp. 65-88, 1988.

[89] H. Khalil, "Nonlinear systems," 2002.

[90] E. Colgate and N. Hogan, "An analysis of contact instability in terms of passive physical equivalents," in Robotics and Automation, 1989. Proceedings., 1989 IEEE International Conference on, pp. 404-409, IEEE, 1989.

[91] K. Ogata, Modern Control Engineering. Instrumentation and controls series, Prentice Hall, 2010.

[92] D. Karnopp, "Computer simulation of stick-slip friction in mechanical dynamic systems," Journal of dynamic systems, measurement, and control, vol. 107, no. 1, pp. 100-103, 1985.

[93] V. Aitken, "Course notes for sysc 5502: Advanced linear systems." Carleton University Lecture, Fall 2013.

[94] M. W. Spong, S. Hutchinson, and M. Vidyasagar, Robot modeling and control, vol. 3. Wiley New York, 2006. 
[95] W. Gander, G. H. Golub, and R. Strebel, "Least-squares fitting of circles and ellipses," BIT Numerical Mathematics, vol. 34, no. 4, pp. 558-578, 1994.

[96] D. Eberly, "Least squares fitting of data." webpage, 2015.

[97] T. A. O. S. Inc., "Tsl250r, tsl251r, tsl252r light-to-voltage optical sensors." online at https://www.ams.com, 2007.

[98] F. Devernay, "C/c++ minpack." https://github.com/devernay/ cminpack, 2007.

[99] H. Olsson, K. J. Åström, C. C. De Wit, M. Gäfvert, and P. Lischinsky, "Friction models and friction compensation," European journal of control, vol. 4, no. 3, pp. 176-195, 1998. 


\section{Appendix A}

\section{Additional Material}

A.1 $X_{R}(s)$ for the isolated system $\left(F_{\text {ext }}=0\right)$ with a PID position controller and nonzero initial conditions

$$
X_{R}(s)=\frac{N_{1} x_{R}(0)+N_{2} x_{S}(0)+N_{3} \dot{x}_{R}(0)+N_{4} \dot{x}_{S}(0)}{D_{1} s^{5}+D_{2} s^{4}+D_{3} s^{3}+D_{4} s^{2}}
$$


where

$$
\begin{aligned}
N_{1}= & M_{R} M_{S} s^{4}+\left(B_{R} M_{S}+K_{d} M_{S}+B_{S} M_{R}+B_{S} M_{S}\right) s^{3} \\
& \ldots+\left(B_{R} B_{S}+K_{S} M_{R}\right) s^{2}+\left(B_{R} K_{S}-B_{S} K_{p}+K_{d} K_{S}\right) s \\
& \ldots-B_{S} K_{I} \\
N_{2}= & \left(K_{p} M_{S}+K_{S} M_{S}\right) s^{2}+\left(B_{S} K_{p}-K_{d} K_{S}+K_{I} M_{S}\right) s+B_{s} K_{I} \\
N_{3}= & M_{R} M_{S} s^{3}+B_{S} M_{R} s^{2}+K_{S} M_{R} s \\
N_{4}= & \left(B_{S} M_{S}+K_{D} M_{S}\right) s^{2}+\left(K_{p} M_{S}+K_{S} M_{S}\right) s+K_{I} M_{S} \\
D_{1}= & M_{R} M_{S} \\
D_{2}= & B_{R} M_{S}+B_{S} M_{R}+B_{S} M_{S}+K_{d} M_{S} \\
D_{3}= & B_{R} B_{S}+K_{S} M_{R}+K_{p} M_{S}+K_{S} M_{S} \\
D_{4}= & B_{R} K_{S}+K_{I} M_{S}
\end{aligned}
$$

\section{A.2 Calibration of damping coefficient for the 1DoF test platform}

To determine an appropriate damping coefficient, the motor arm was commanded to move at a constant velocity and the mean torque command was calculated. The results of the experiment can be seen in Figure A.1. It is noted that the torque command did tend to follow a linear trend with a slope of $B=4.05 \mathrm{~N} \mathrm{~s}$; however,

there is significant deviation. For this reason, the relationship was investigated further by studying the response of the system to an initial velocity.

To measure the response to an initial velocity input, the test platform was 


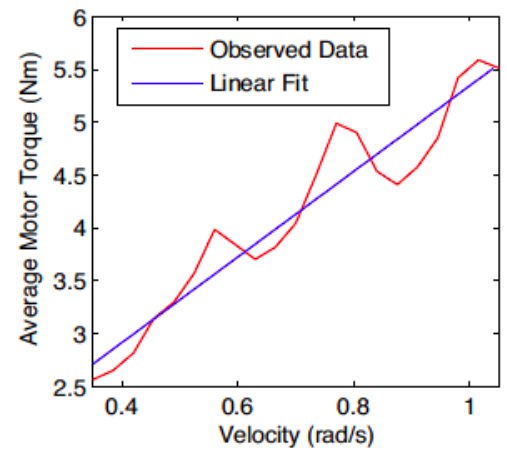

Figure A.1: Labelled photograph of commanded torque versus output velocity to determine system damping

commanded to perform a $90^{\circ}$ rotation at a constant velocity. At $90^{\circ}$, the motor was disabled and the position was recorded using the encoder. To determine if applied load affected friction, these experiments were performed with and without the arm and with an additional added mass to the arm. The position response was then fit using a linear model that assumed a constant applied dry friction and weight torques, and a nonlinear model that assumed the dry friction to be a constant torque with a sinusoidal torque due to weight. The linear and nonlinear models are summarized by the equations,

$$
\begin{aligned}
& J \ddot{\theta}+B \dot{\theta}=-F_{f} \\
& J \ddot{\theta}+B \dot{\theta}=W \sin \left(\theta-\theta_{o}\right)-F_{f}
\end{aligned}
$$

To fit the linear model, the following curve was fit to the observed position data,

$$
\theta=\left(v(0)-\frac{a}{\tau}\right) \Delta t+a-a e^{\frac{t}{\tau}}
$$




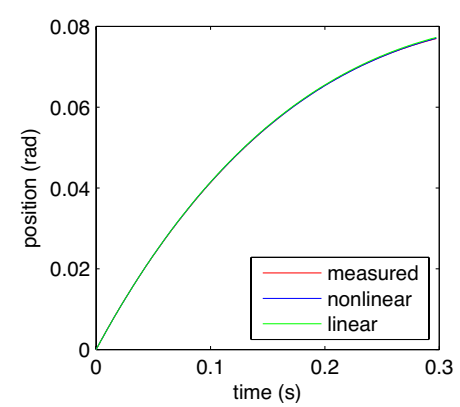

(a)

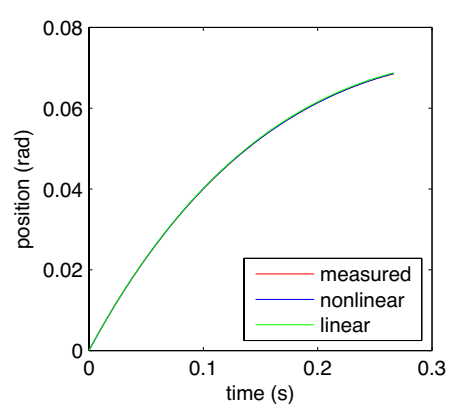

(b)

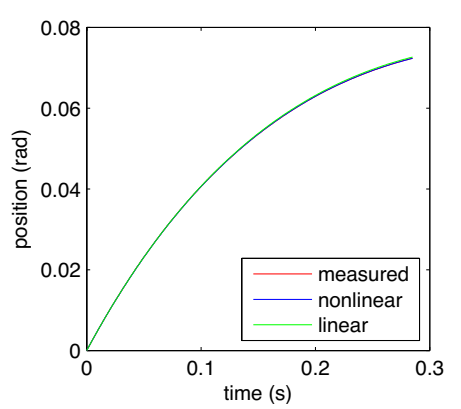

(c)

Figure A.2: Curve fit of system response to an initial velocity of $30^{\circ}$ (a) without the arm, (b) with the arm, and (c) with the arm and an added mass.

with the $\tau$ and $a$ parameters fit. The nonlinear model was fit using the numerical solution of (A.3) generated using Matlab's ode45 solver with the ratios $\frac{B}{J}$, $\frac{W}{B}, \frac{F_{f}}{J}$ and $\theta_{o}$. Sample curve fits are provided in Figure A.2 and it can be seen that the current models fit the trend well, with a maximum root mean squared error of $0.1129 \mathrm{rad}$. From the models, it is seen that friction appears to increase with weight and has an average damping coefficient of $5.1 \mathrm{Nms}$ across all trials. With this damping coefficient, the linear model for the actuator and arm is approximated as,

$$
1.363 \mathrm{~kg} \mathrm{~m}^{2} \ddot{\theta}+5.1 \mathrm{Nms} \dot{\theta}=M_{e x t}
$$

\section{A.3 Calibration of damping coefficient for the 1DoF CLS}

To estimate the damping coefficient of the 1DoF CLS, the response of the system to initial conditions was fit with a linear model mass-spring-damper system with 
an initial position deviation and constant applied force. The constant applied force accounts for the weight of the shell and an assumed Coulomb friction force. The model was provided with the mass and spring constant; only the applied force and damping were varied in curve fitting.

To measure the system response, masses were hung from the CLS and the external shell was lifted and released. The potentiometer output was sampled at $2 \mathrm{kHz}$ and the responses were assumed to begin the sample before the estimated velocity deviated from zero and lasted until the sensor displaced to $95 \%$ of the steady state displacement. Sample responses and fits are provided in Fig. A.3. In determining the damping coefficient, only trials where the linear model was able to minimize the root mean squared error below a threshold of $0.1 \mathrm{~mm}$ were considered. As can be seen in Figure A.3, the model appears to capture the response of the system when a negative damping coefficient is used. It is believed the negative damping may be capturing the difference between stiction and sliding friction [99], or the dry friction may vary with position or loading.

From the data, it is clear the dominant form of friction is not viscous. In light of this, a damping coefficient is estimated by averaging the ratio of the estimated dry friction to velocity for a range of expected velocities, excluding very low velocities. Dry friction in the sensor is estimated as the smallest weight in the force calibration of the spring that caused a measurable deformation, $F_{f}=3.647 \mathrm{~N}$. Assuming a range of velocities from $1 \mathrm{~mm} \mathrm{~s}^{-1}$ to $300 \mathrm{~mm} \mathrm{~s}^{-1}$, the average damping coefficient is be $B=20.8017 \mathrm{~N} \mathrm{~s} \mathrm{~m}^{-1}$. 


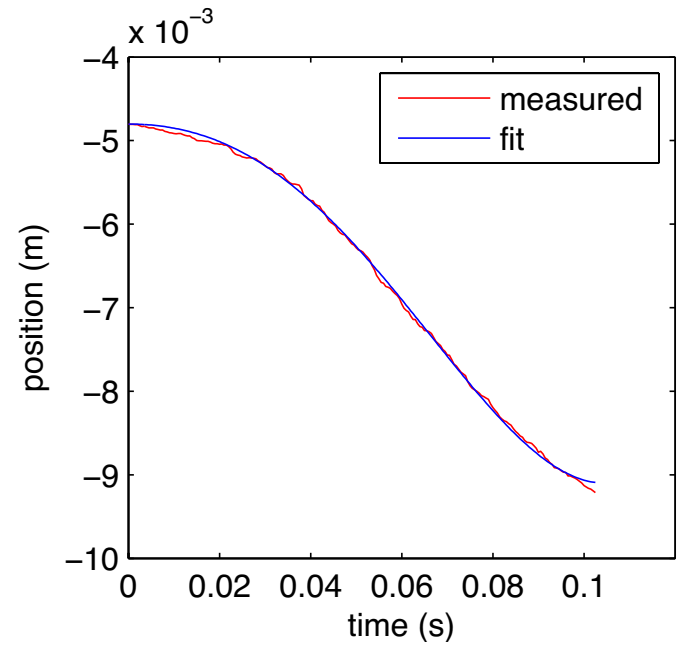

(a)

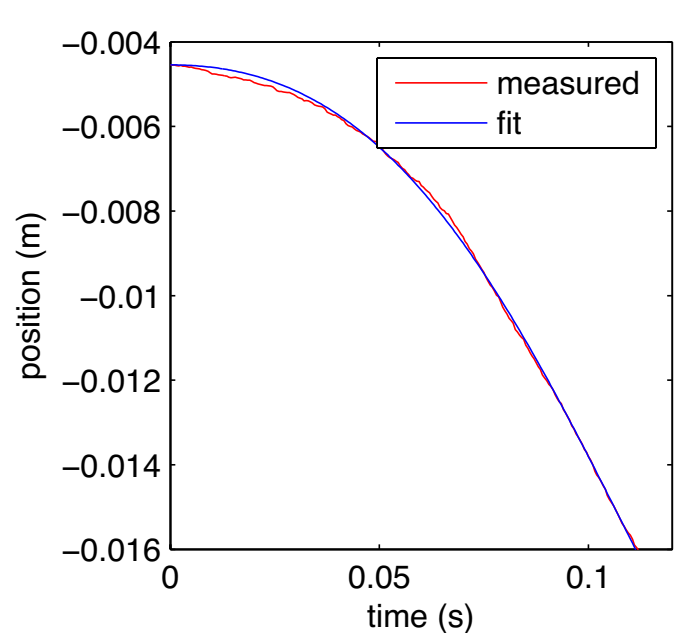

(b)

Figure A.3: Sample CLS model fit curves for added masses (a) $M=$ $1.027 \mathrm{~kg}$ and (b) $M=1.985 \mathrm{~kg}$

\section{A.4 Parameters for Head Impact Model}

The parameters employed for the head impact model can be found in Table A.1 below. Many parameters were taken directly from the paper [30]. 
Table A.1: Selected parameters for the head impact model

\begin{tabular}{|l|l|}
\hline$n_{c s}$ & 1.65 \\
\hline$n_{c b}$ & 1.8 \\
\hline$n_{r b}$ & 2.65 \\
\hline$b_{s}$ & $0.003 \mathrm{~m}$ \\
\hline$b_{s m}$ & $0.0024 \mathrm{~m}$ \\
\hline$b_{c}$ & $0 \mathrm{~m}$ \\
\hline$b_{c m}$ & $0 \mathrm{~m}$ \\
\hline$R_{r}$ & $1 \times 10^{9} \mathrm{~m}$ \\
\hline$R_{c}$ & $1 \times 10^{9} \mathrm{~m}$ \\
\hline$R_{b}$ & $0.075 \mathrm{~m}$ \\
\hline$R_{s}$ & $0.078 \mathrm{~m}$ \\
\hline$m_{h}$ & $4.5 \mathrm{~kg}$ \\
\hline$E_{b}$ & $6.5 \mathrm{GPa}$ \\
\hline$E_{s}$ & $16.7 \mathrm{MPa}$ \\
\hline$E_{r}$ & $69 \mathrm{GPa}$ \\
\hline$E_{c}$ & $69 \mathrm{GPa}$ \\
\hline$\nu_{b}$ & 0.22 \\
\hline$\nu_{s}$ & 0.42 \\
\hline$\nu_{r}$ & 0.33 \\
\hline$\nu_{c}$ & 0.33 \\
\hline
\end{tabular}




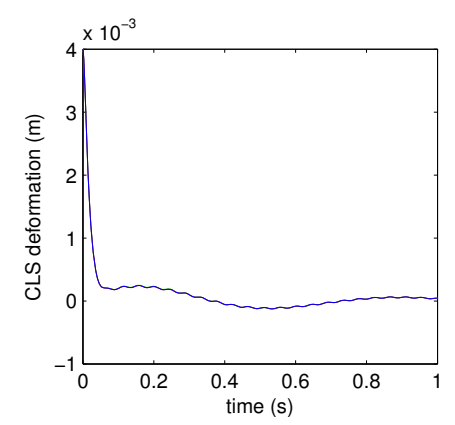

(a) $K_{C}=100 \mathrm{~N} \mathrm{~m}^{-1}$,

$B_{C}=\mathbf{0}$,

$M_{E}=0.01 \mathrm{~kg}$,

$K_{E}=100 \mathrm{~N} \mathrm{~m}^{-1}$ \&

$B_{E}=\mathbf{0}$

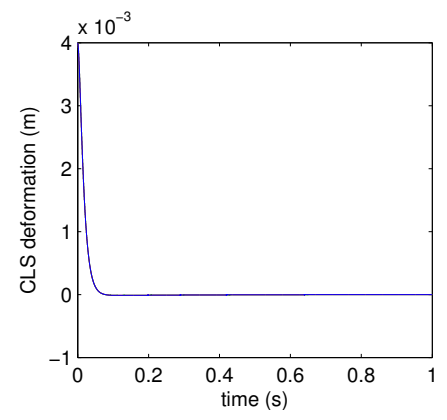

(d) $K_{C}=1000000 \mathrm{~N} \mathrm{~m}^{-1},(\mathrm{e})$

$$
\begin{aligned}
& B_{C}=\mathbf{0}, \\
& M_{E}=0.01 \mathrm{~kg}, \\
& K_{E}=\mathbf{0} \& B_{E}=\mathbf{0}
\end{aligned}
$$

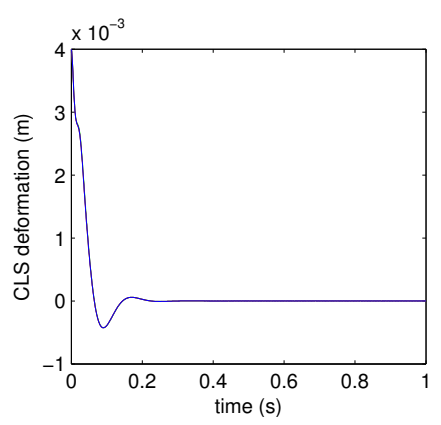

(b) $K_{C}=10000 \mathrm{~N} \mathrm{~m}^{-1}$,

$B_{C}=\mathbf{0}$,

$M_{E}=0.01 \mathrm{~kg}$,

$K_{E}=10000 \mathrm{~N} \mathrm{~m}^{-1}$

\& $B_{E}=\mathbf{0}$

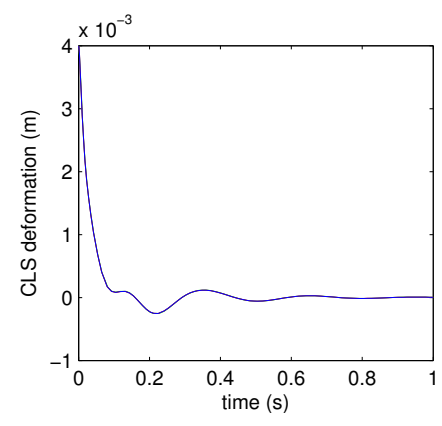

(e) $K_{C}=1000 \mathrm{~N} \mathrm{~m}^{-1}$,

$$
B_{C}=10 \mathrm{~N} \mathrm{~s} \mathrm{~m}^{-1} \text {, }
$$

$M_{E}=1 \mathrm{~kg}$,

$K_{E}=1000 \mathrm{Nm}^{-1} \&$

$B_{E}=10 \mathrm{Ns} \mathrm{m}^{-1}$

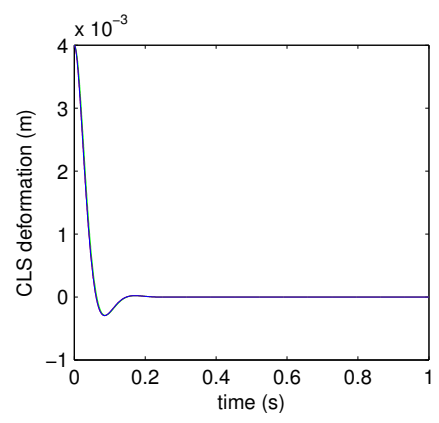

(c) $K_{C}=1000000 \mathrm{~N} \mathrm{~m}^{-1}$,

$B_{C}=\mathbf{0}$,

$M_{E}=100 \mathrm{~kg}$,

$K_{E}=\mathbf{0} \& B_{E}=\mathbf{0}$

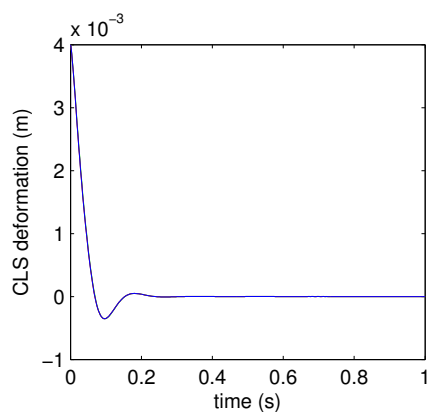

(f) $K_{C}=10000 \mathrm{~N} \mathrm{~m}^{-1}$, $B_{C}=100 \mathrm{~N} \mathrm{~s} \mathrm{~m}^{-1}$, $M_{E}=1 \mathrm{~kg}$, $K_{E}=10000 \mathrm{~N} \mathrm{~m}^{-1}$ \& $B_{E}=100 \mathrm{~N} \mathrm{~s} \mathrm{~m}^{-1}$

Figure A.4: CLS deflection results of the controlled system with varying imposed saturation forces interacting with the environment. The blue, red and green lines indicate $166.34 \mathrm{~N}, 100 \mathrm{~N}$ and $50 \mathrm{~N}$ saturation forces, respectively. 


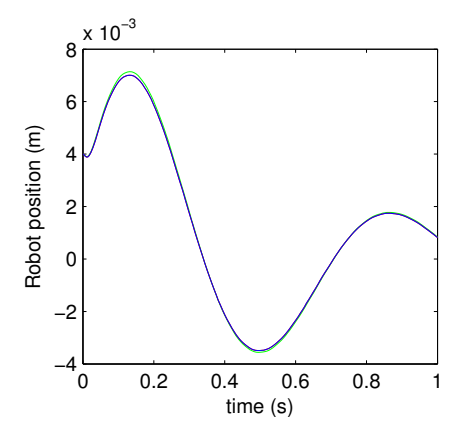

(a) $K_{C}=100 \mathrm{~N} \mathrm{~m}^{-1}$,

$B_{C}=\mathbf{0}$,

$M_{E}=0.01 \mathrm{~kg}$,

$K_{E}=100 \mathrm{~N} \mathrm{~m}^{-1}$ \&

$B_{E}=\mathbf{0}$

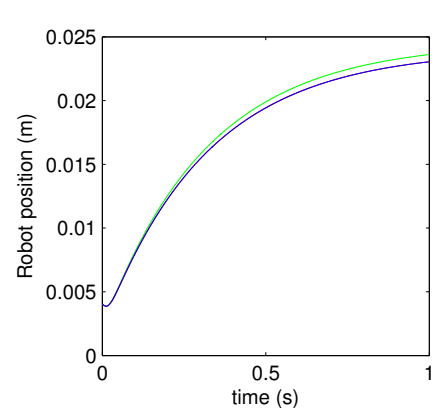

(d) $K_{C}=1000000 \mathrm{~N} \mathrm{~m}^{-1},(\mathrm{e})$

$$
\begin{aligned}
& B_{C}=\mathbf{0}, \\
& M_{E}=0.01 \mathrm{~kg}, \\
& K_{E}=\mathbf{0} \& B_{E}=\mathbf{0}
\end{aligned}
$$

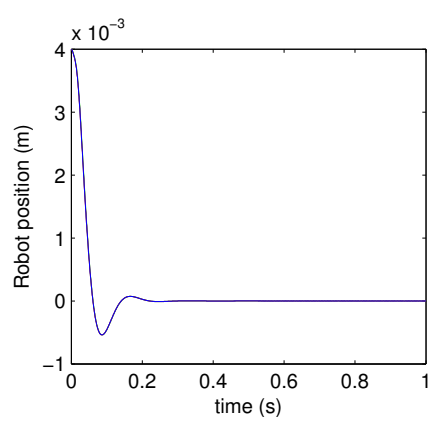

(b) $K_{C}=10000 \mathrm{~N} \mathrm{~m}^{-1}$, $B_{C}=\mathbf{0}$, $M_{E}=0.01 \mathrm{~kg}$, $K_{E}=10000 \mathrm{~N} \mathrm{~m}^{-1}$ \& $B_{E}=\mathbf{0}$

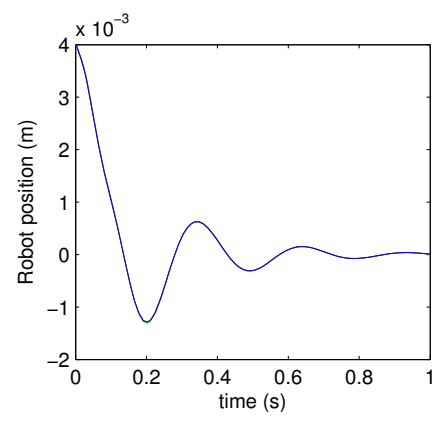

(e) $K_{C}=1000 \mathrm{~N} \mathrm{~m}^{-1}$,

$$
\begin{aligned}
& B_{C}=10 \mathrm{~N} \mathrm{~s} \mathrm{~m}^{-1}, \\
& M_{E}=1 \mathrm{~kg}, \\
& K_{E}=1000 \mathrm{~N} \mathrm{~m}^{-1} \& \\
& B_{E}=10 \mathrm{~N} \mathrm{~s} \mathrm{~m}^{-1}
\end{aligned}
$$

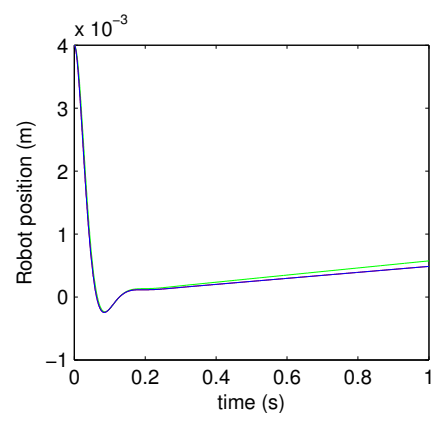

(c) $K_{C}=1000000 \mathrm{~N} \mathrm{~m}^{-1}$, $B_{C}=\mathbf{0}$, $M_{E}=100 \mathrm{~kg}$, $K_{E}=\mathbf{0} \& B_{E}=\mathbf{0}$

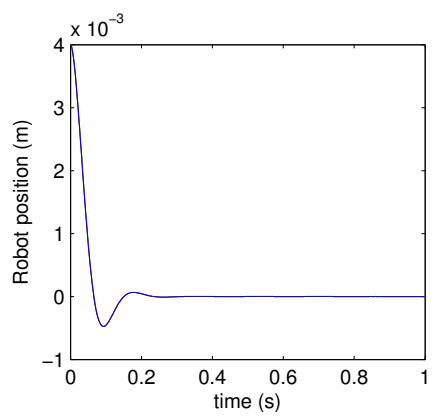

(f) $K_{C}=10000 \mathrm{~N} \mathrm{~m}^{-1}$, $B_{C}=100 \mathrm{~N} \mathrm{~s} \mathrm{~m}^{-1}$, $M_{E}=1 \mathrm{~kg}$, $K_{E}=10000 \mathrm{~N} \mathrm{~m}^{-1}$ \& $B_{E}=100 \mathrm{~N} \mathrm{~s} \mathrm{~m}^{-1}$

Figure A.5: Robot motion results of the controlled system with varying saturation forces interacting with the environment. The blue, red and green lines indicate $166.34 \mathrm{~N}, 100 \mathrm{~N}$ and $50 \mathrm{~N}$ saturation forces, respectively. 


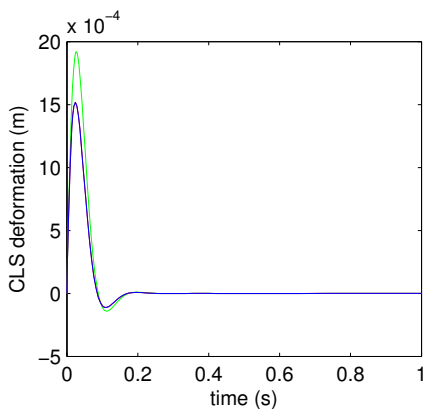

(a) $V_{R}(0)=0.15 \mathrm{~m} \mathrm{~s}^{-1}$

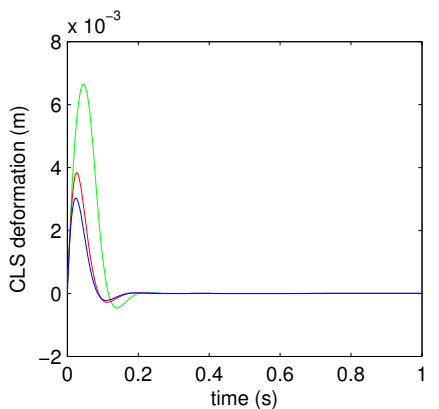

(b) $V_{R}(0)=0.3 \mathrm{~m} \mathrm{~s}^{-1}$

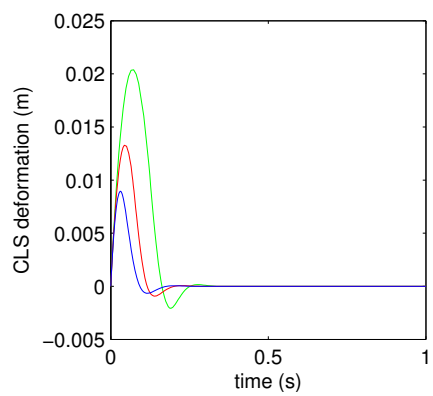

(c) $V_{R}(0)=0.6 \mathrm{~m} \mathrm{~s}^{-1}$

Figure A.6: CLS deflection results of the controlled system with varying saturation forces impacting a rigid environment. The blue, red and green lines indicate $166.34 \mathrm{~N}, 100 \mathrm{~N}$ and $50 \mathrm{~N}$ saturation forces, respectively.

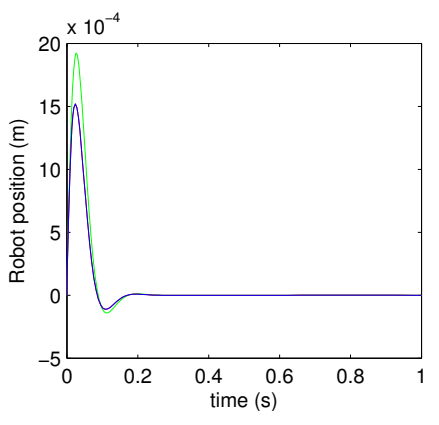

(a) $V_{R}(0)=0.15 \mathrm{~m} \mathrm{~s}^{-1}$

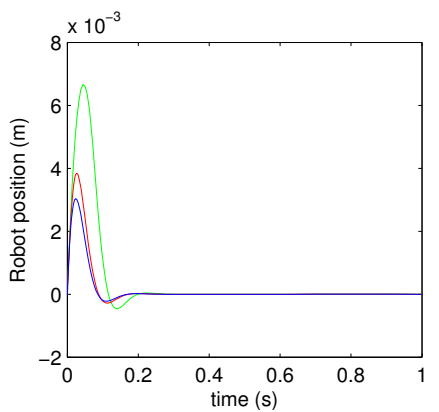

(b) $V_{R}(0)=0.3 \mathrm{~m} \mathrm{~s}^{-1}$

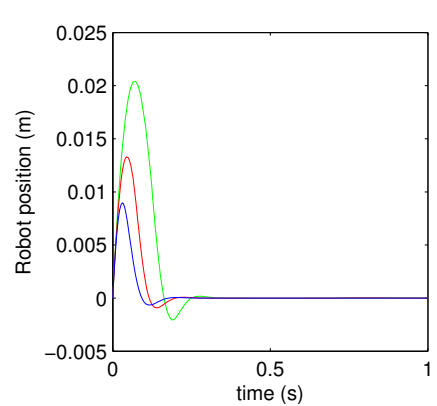

(c) $V_{R}(0)=0.6 \mathrm{~m} \mathrm{~s}^{-1}$

Figure A.7: Robot motion results of the controlled system with varying saturation forces impacting a rigid environment. The blue, red and green lines indicate $166.34 \mathrm{~N}, 100 \mathrm{~N}$ and $50 \mathrm{~N}$ saturation forces, respectively. 


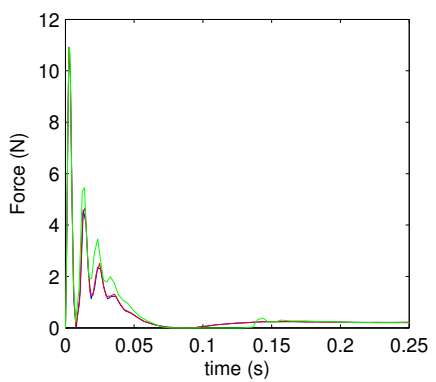

(a) $V_{H}(0)=0.15 \mathrm{~m} \mathrm{~s}^{-1}$

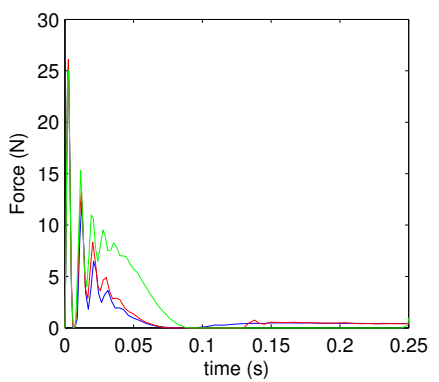

(b) $V_{H}(0)=0.3 \mathrm{~m} \mathrm{~s}^{-1}$

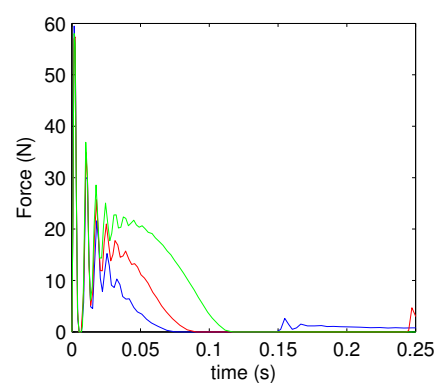

(c) $V_{H}(0)=0.6 \mathrm{~m} \mathrm{~s}^{-1}$

Figure A.8: Contact force results of the controlled system with varying saturation forces impacting the head model. The blue, red and green lines indicate $166.34 \mathrm{~N}, 100 \mathrm{~N}$ and $50 \mathrm{~N}$ saturation forces, respectively.

\section{A.5 Additional Simulation Results:}




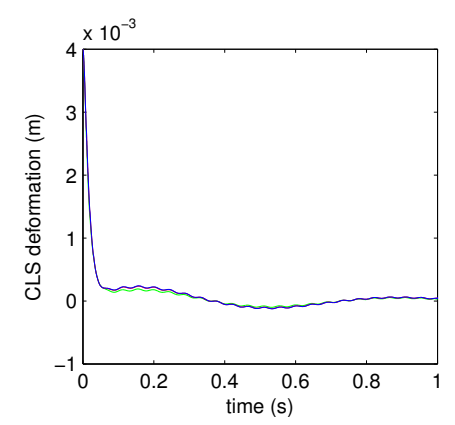

(a) $K_{C}=100 \mathrm{~N} \mathrm{~m}^{-1}$,

$B_{C}=\mathbf{0}$,

$M_{E}=0.01 \mathrm{~kg}$,

$K_{E}=100 \mathrm{~N} \mathrm{~m}^{-1}$ \&

$B_{E}=\mathbf{0}$

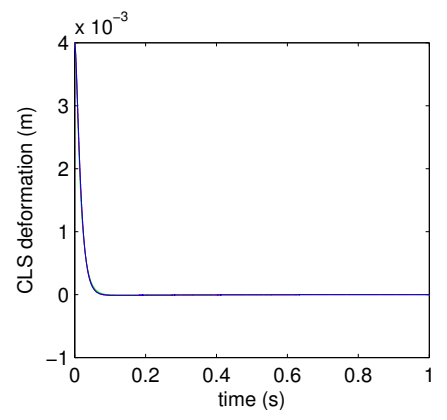

(d) $K_{C}=1000000 \mathrm{~N} \mathrm{~m}^{-1},(\mathrm{e})$

$$
\begin{aligned}
& B_{C}=\mathbf{0}, \\
& M_{E}=0.01 \mathrm{~kg}, \\
& K_{E}=\mathbf{0} \& B_{E}=\mathbf{0}
\end{aligned}
$$

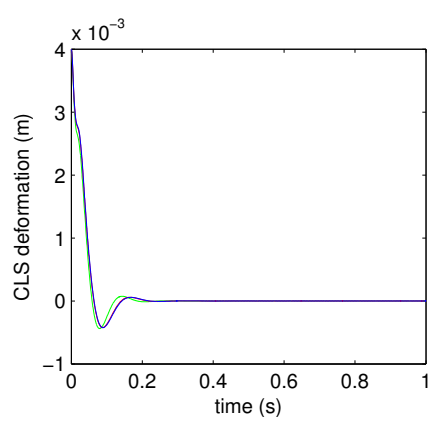

(b) $K_{C}=10000 \mathrm{~N} \mathrm{~m}^{-1}$,

$B_{C}=\mathbf{0}$,

$M_{E}=0.01 \mathrm{~kg}$,

$K_{E}=10000 \mathrm{~N} \mathrm{~m}^{-1}$

\& $B_{E}=\mathbf{0}$

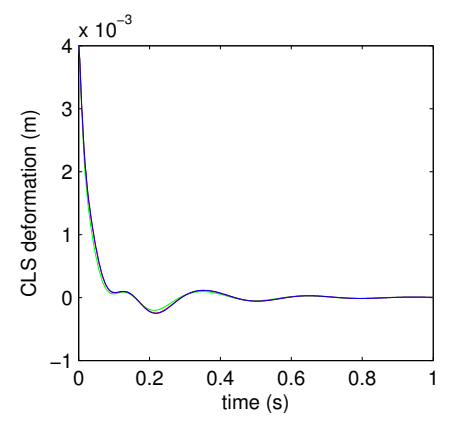

(e) $K_{C}=1000 \mathrm{~N} \mathrm{~m}^{-1}$,

$$
B_{C}=10 \mathrm{~N} \mathrm{~s} \mathrm{~m}^{-1} \text {, }
$$

$M_{E}=1 \mathrm{~kg}$,

$K_{E}=1000 \mathrm{Nm}^{-1} \&$

$B_{E}=10 \mathrm{Ns} \mathrm{m}^{-1}$

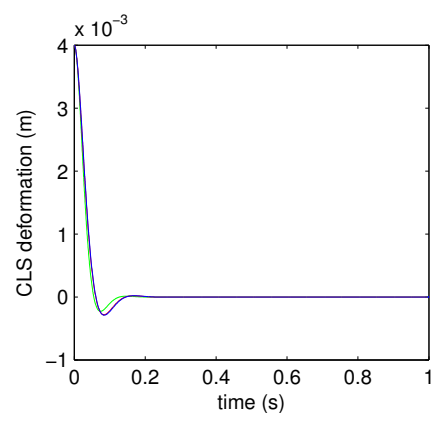

(c) $K_{C}=1000000 \mathrm{~N} \mathrm{~m}^{-1}$,

$B_{C}=\mathbf{0}$,

$M_{E}=100 \mathrm{~kg}$,

$K_{E}=\mathbf{0} \& B_{E}=\mathbf{0}$

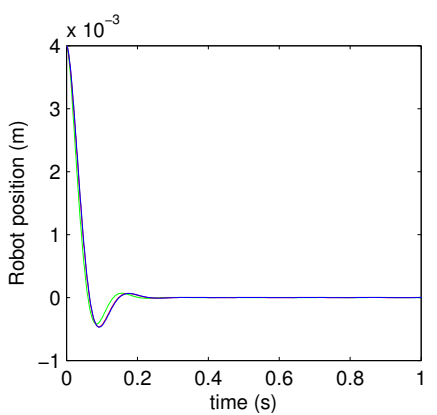

(f) $K_{C}=10000 \mathrm{~N} \mathrm{~m}^{-1}$, $B_{C}=100 \mathrm{~N} \mathrm{~s} \mathrm{~m}^{-1}$, $M_{E}=1 \mathrm{~kg}$, $K_{E}=10000 \mathrm{~N} \mathrm{~m}^{-1}$ \& $B_{E}=100 \mathrm{~N} \mathrm{~s} \mathrm{~m}^{-1}$

Figure A.9: CLS deflection results of the discretely controlled system with varying sampling frequencies interacting with the environment. The blue, red and green lines indicate $2 \mathrm{kHz}, 1 \mathrm{kHz}$ and $200 \mathrm{~Hz}$ sampling frequencies, respectively. 


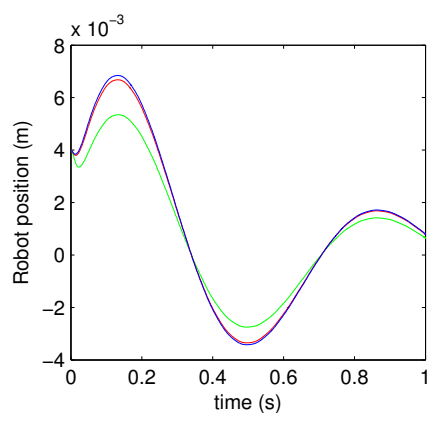

(a) $K_{C}=100 \mathrm{~N} \mathrm{~m}^{-1}$,

$B_{C}=\mathbf{0}$,

$M_{E}=0.01 \mathrm{~kg}$,

$K_{E}=100 \mathrm{~N} \mathrm{~m}^{-1}$ \&

$B_{E}=\mathbf{0}$

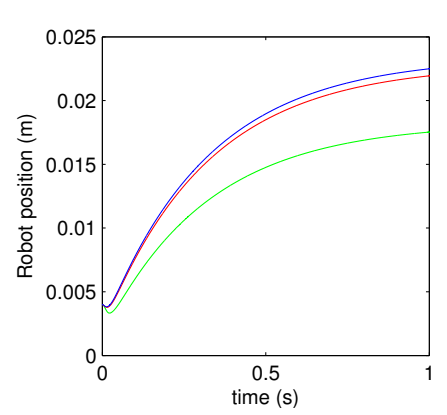

(d) $K_{C}=1000000 \mathrm{~N} \mathrm{~m}^{-1},(\mathrm{e})$

$$
\begin{aligned}
& B_{C}=\mathbf{0}, \\
& M_{E}=0.01 \mathrm{~kg}, \\
& K_{E}=\mathbf{0} \& B_{E}=\mathbf{0}
\end{aligned}
$$

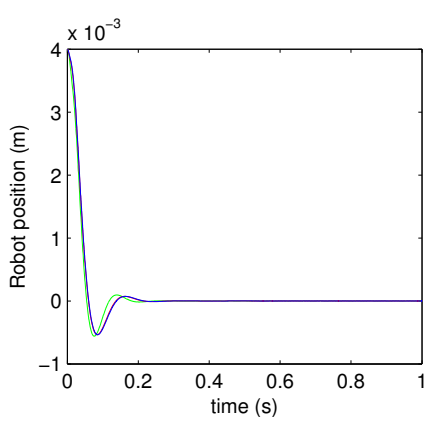

(b) $K_{C}=10000 \mathrm{~N} \mathrm{~m}^{-1}$,

$B_{C}=\mathbf{0}$,

$M_{E}=0.01 \mathrm{~kg}$,

$K_{E}=10000 \mathrm{~N} \mathrm{~m}^{-1}$

\& $B_{E}=\mathbf{0}$

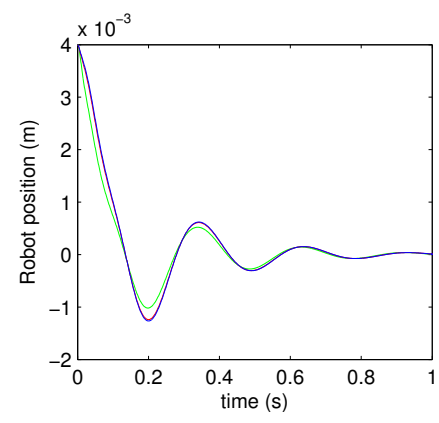

(e) $K_{C}=1000 \mathrm{~N} \mathrm{~m}^{-1}$,

$$
B_{C}=10 \mathrm{~N} \mathrm{~s} \mathrm{~m}^{-1} \text {, }
$$

$M_{E}=1 \mathrm{~kg}$,

$K_{E}=1000 \mathrm{Nm}^{-1} \&$

$B_{E}=10 \mathrm{~N} \mathrm{~s} \mathrm{~m}^{-1}$

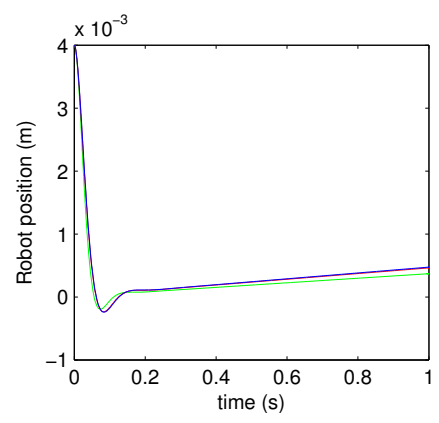

(c) $K_{C}=1000000 \mathrm{~N} \mathrm{~m}^{-1}$,

$B_{C}=\mathbf{0}$,

$M_{E}=100 \mathrm{~kg}$,

$K_{E}=\mathbf{0} \& B_{E}=\mathbf{0}$

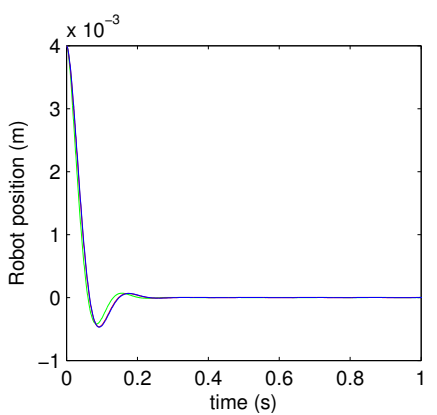

(f) $K_{C}=10000 \mathrm{~N} \mathrm{~m}^{-1}$, $B_{C}=100 \mathrm{~N} \mathrm{~s} \mathrm{~m}^{-1}$, $M_{E}=1 \mathrm{~kg}$, $K_{E}=10000 \mathrm{~N} \mathrm{~m}^{-1}$ \& $B_{E}=100 \mathrm{~N} \mathrm{~s} \mathrm{~m}^{-1}$

Figure A.10: Robot motion results of the discretely controlled system with varying sampling frequencies interacting with the environment. The blue, red and green lines indicate $2 \mathrm{kHz}, 1 \mathrm{kHz}$ and $200 \mathrm{~Hz}$ sampling frequencies, respectively. 


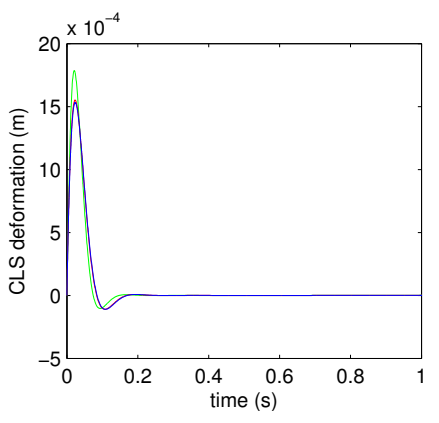

(a) $V_{R}(0)=0.15 \mathrm{~m} \mathrm{~s}^{-1}$

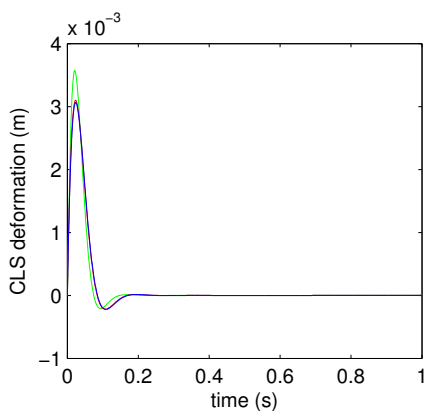

(b) $V_{R}(0)=0.3 \mathrm{~m} \mathrm{~s}^{-1}$

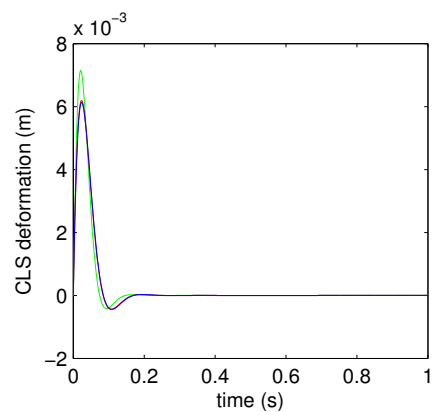

(c) $V_{R}(0)=0.6 \mathrm{~m} \mathrm{~s}^{-1}$

Figure A.11: CLS deflection results of the discretely controlled system with varying sampling frequencies impacting a rigid environment. The blue, red and green lines indicate $2 \mathrm{kHz}, 1 \mathrm{kHz}$ and $200 \mathrm{~Hz}$ sampling frequencies, respectively.

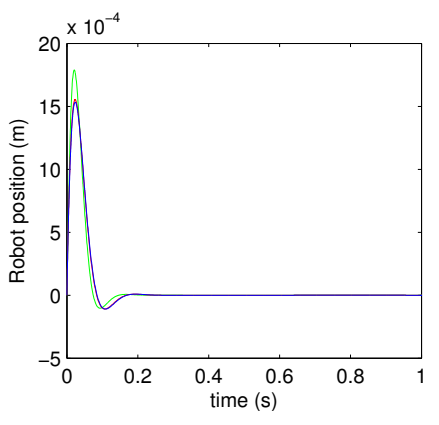

(a) $V_{R}(0)=0.15 \mathrm{~m} \mathrm{~s}^{-1}$

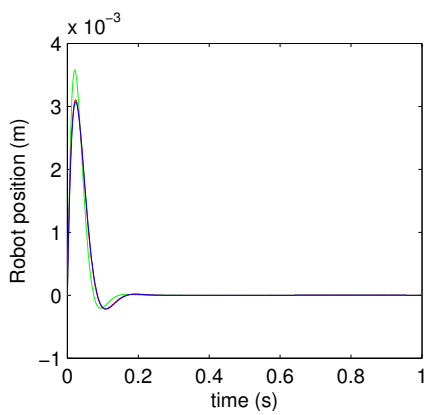

(b) $V_{R}(0)=0.3 \mathrm{~m} \mathrm{~s}^{-1}$

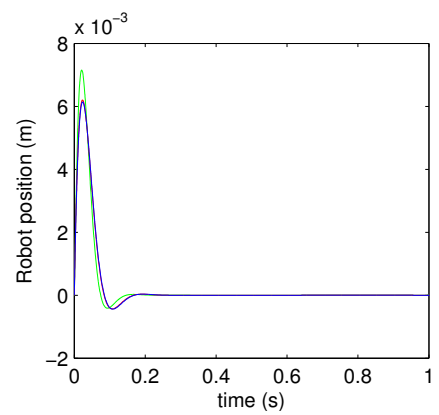

(c) $V_{R}(0)=0.6 \mathrm{~m} \mathrm{~s}^{-1}$

Figure A.12: Robot motion results of the discretely controlled system with varying sampling frequencies impacting a rigid environment. The blue, red and green lines indicate $2 \mathrm{kHz}, 1 \mathrm{kHz}$ and $200 \mathrm{~Hz}$ sampling frequencies, respectively. 


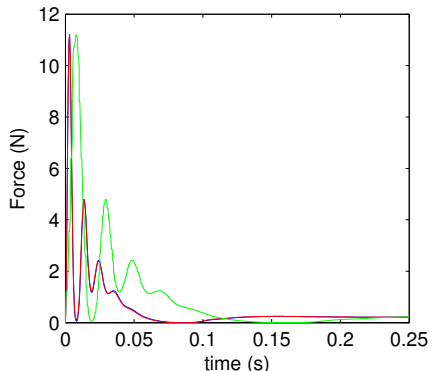

(a) $V_{H}(0)=0.15 \mathrm{~m} \mathrm{~s}^{-1}$

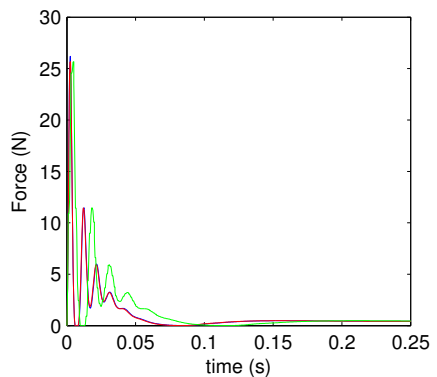

(b) $V_{H}(0)=0.3 \mathrm{~m} \mathrm{~s}^{-1}$

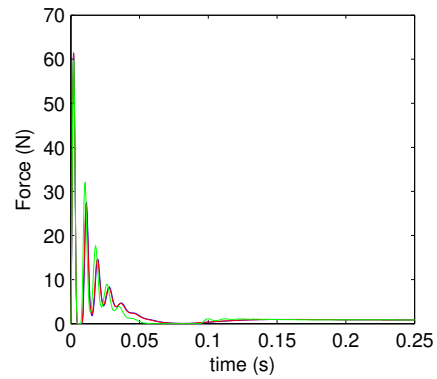

(c) $V_{H}(0)=0.6 \mathrm{~m} \mathrm{~s}^{-1}$

Figure A.13: Contact force results of the discretely controlled system with varying sampling frequencies impacting the head model. The blue, red and green lines indicate $2 \mathrm{kHz}, 1 \mathrm{kHz}$ and $200 \mathrm{~Hz}$ sampling frequencies, respectively. 


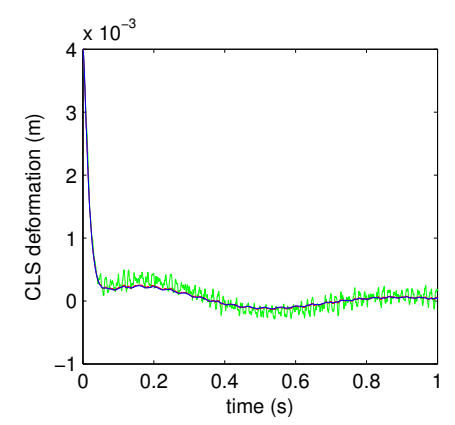

(a) $K_{C}=100 \mathrm{~N} \mathrm{~m}^{-1}$,

$B_{C}=\mathbf{0}$,

$M_{E}=0.01 \mathrm{~kg}$,

$K_{E}=100 \mathrm{~N} \mathrm{~m}^{-1}$ \&

$B_{E}=\mathbf{0}$

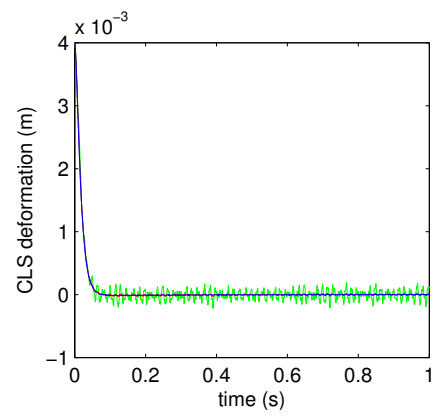

(d) $K_{C}=1000000 \mathrm{~N} \mathrm{~m}^{-1}$, (e)

$$
\begin{aligned}
& B_{C}=\mathbf{0}, \\
& M_{E}=0.01 \mathrm{~kg}, \\
& K_{E}=\mathbf{0} \& B_{E}=\mathbf{0}
\end{aligned}
$$

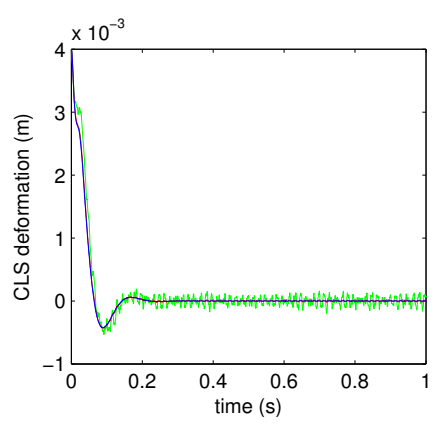

(b) $K_{C}=10000 \mathrm{~N} \mathrm{~m}^{-1}$,

$B_{C}=\mathbf{0}$,

$M_{E}=0.01 \mathrm{~kg}$,

$K_{E}=10000 \mathrm{~N} \mathrm{~m}^{-1}$

\& $B_{E}=\mathbf{0}$

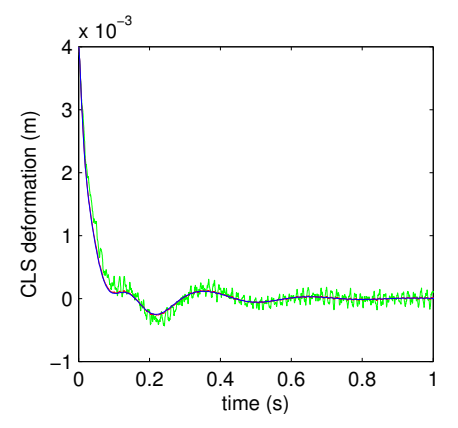

(e) $K_{C}=1000 \mathrm{~N} \mathrm{~m}^{-1}$,

$$
\begin{aligned}
& B_{C}=10 \mathrm{~N} \mathrm{~s} \mathrm{~m}^{-1} \\
& M_{E}=1 \mathrm{~kg}, \\
& K_{E}=1000 \mathrm{~N} \mathrm{~m}^{-1} \& \\
& B_{E}=10 \mathrm{~N} \mathrm{~s} \mathrm{~m}^{-1}
\end{aligned}
$$

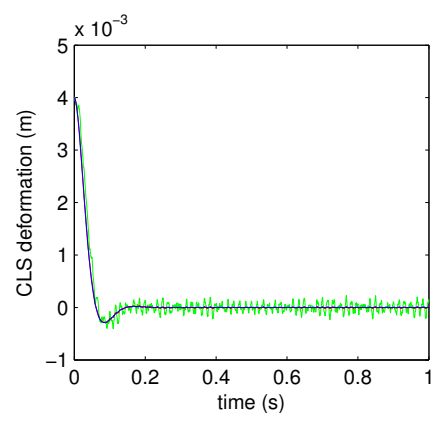

(c) $K_{C}=1000000 \mathrm{~N} \mathrm{~m}^{-1}$, $B_{C}=\mathbf{0}$, $M_{E}=100 \mathrm{~kg}$, $K_{E}=\mathbf{0} \& B_{E}=\mathbf{0}$

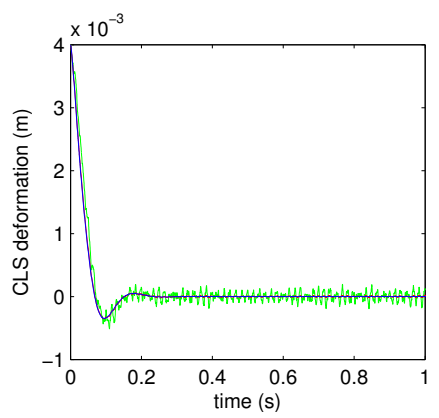

(f) $K_{C}=10000 \mathrm{~N} \mathrm{~m}^{-1}$, $B_{C}=100 \mathrm{~N} \mathrm{~s} \mathrm{~m}^{-1}$, $M_{E}=1 \mathrm{~kg}$, $K_{E}=10000 \mathrm{~N} \mathrm{~m}^{-1}$ \& $B_{E}=100 \mathrm{~N} \mathrm{~s} \mathrm{~m}^{-1}$

Figure A.14: CLS motion results of the discretely controlled system with varying levels of sensor noise interacting with the environment. The blue, red and green lines indicate the measured noise, $10 \times$ the measured noise and $100 \times$ the measured noise, respectively. 


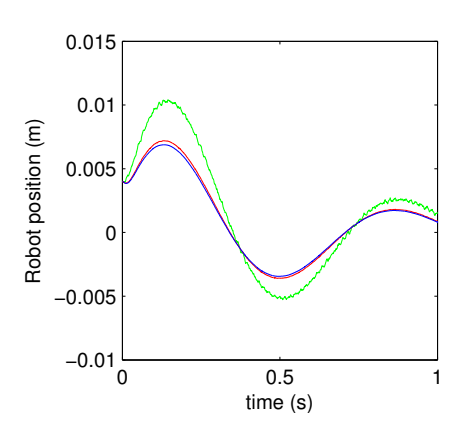

(a) $K_{C}=100 \mathrm{~N} \mathrm{~m}^{-1}$,

$B_{C}=\mathbf{0}$,

$M_{E}=0.01 \mathrm{~kg}$,

$K_{E}=100 \mathrm{~N} \mathrm{~m}^{-1}$ \&

$B_{E}=\mathbf{0}$

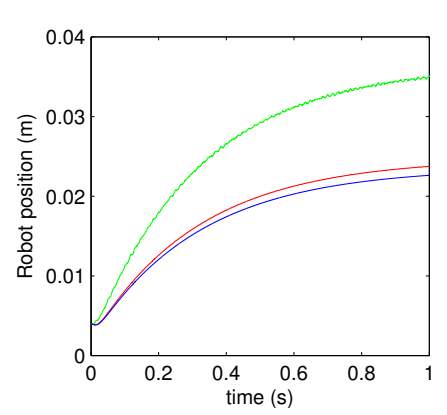

(d) $K_{C}=1000000 \mathrm{~N} \mathrm{~m}^{-1}$, (e)

$$
\begin{aligned}
& B_{C}=\mathbf{0}, \\
& M_{E}=0.01 \mathrm{~kg}, \\
& K_{E}=\mathbf{0} \& B_{E}=\mathbf{0}
\end{aligned}
$$

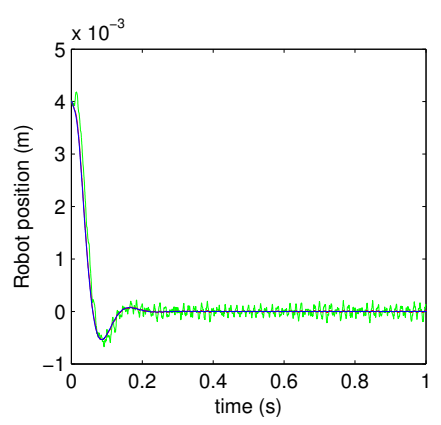

(b) $K_{C}=10000 \mathrm{~N} \mathrm{~m}^{-1}$,

$B_{C}=\mathbf{0}$,

$M_{E}=0.01 \mathrm{~kg}$,

$K_{E}=10000 \mathrm{~N} \mathrm{~m}^{-1}$

\& $B_{E}=\mathbf{0}$

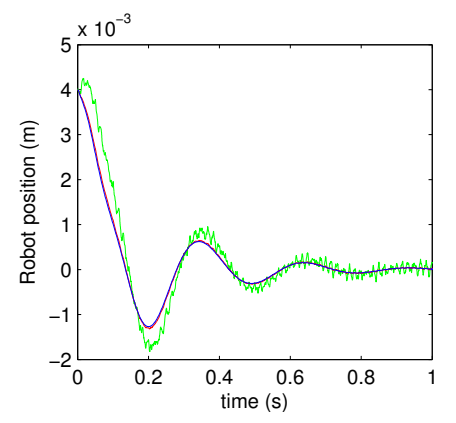

(e) $K_{C}=1000 \mathrm{~N} \mathrm{~m}^{-1}$,

$$
B_{C}=10 \mathrm{~N} \mathrm{~s} \mathrm{~m}^{-1} \text {, }
$$

$M_{E}=1 \mathrm{~kg}$,

$K_{E}=1000 \mathrm{Nm}^{-1} \&$

$B_{E}=10 \mathrm{Ns} \mathrm{m}^{-1}$

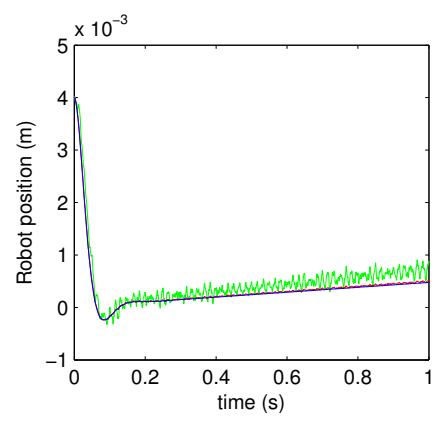

(c) $K_{C}=1000000 \mathrm{~N} \mathrm{~m}^{-1}$,

$B_{C}=\mathbf{0}$,

$M_{E}=100 \mathrm{~kg}$,

$K_{E}=\mathbf{0} \& B_{E}=\mathbf{0}$

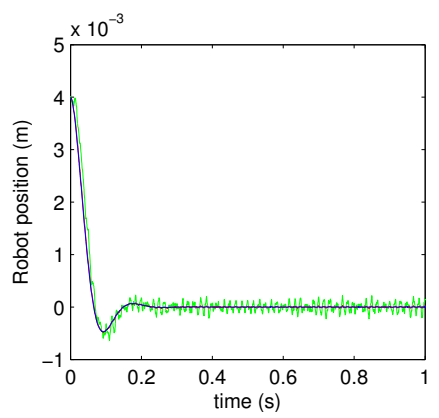

(f) $K_{C}=10000 \mathrm{~N} \mathrm{~m}^{-1}$, $B_{C}=100 \mathrm{~N} \mathrm{~s} \mathrm{~m}^{-1}$, $M_{E}=1 \mathrm{~kg}$, $K_{E}=10000 \mathrm{~N} \mathrm{~m}^{-1}$ \& $B_{E}=100 \mathrm{~N} \mathrm{~s} \mathrm{~m}^{-1}$

Figure A.15: Robot motion results of the discretely controlled system with varying levels of sensor noise interacting with the environment. The blue, red and green lines indicate the measured noise, $10 \times$ the measured noise and $100 \times$ the measured noise, respectively. 


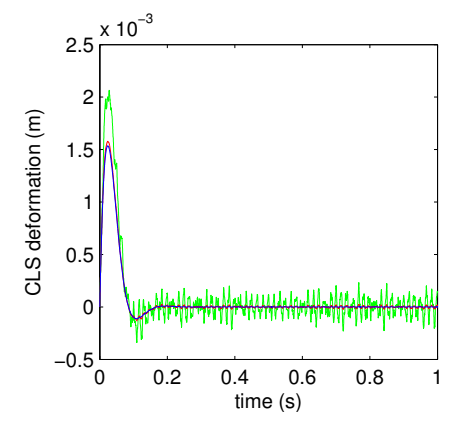

(a) $V_{R}(0)=0.15 \mathrm{~m} \mathrm{~s}^{-1}$

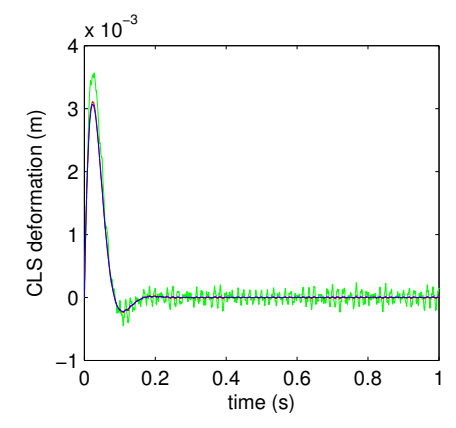

(b) $V_{R}(0)=0.3 \mathrm{~m} \mathrm{~s}^{-1}$

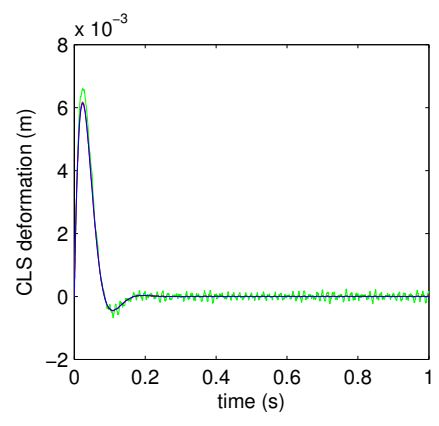

(c) $V_{R}(0)=0.6 \mathrm{~m} \mathrm{~s}^{-1}$

Figure A.16: CLS deflection results of the discretely controlled system with levels of sensor noise impacting a rigid environment. The blue, red and green lines indicate the measured noise, $10 \times$ the measured noise and $100 \times$ the measured noise, respectively.

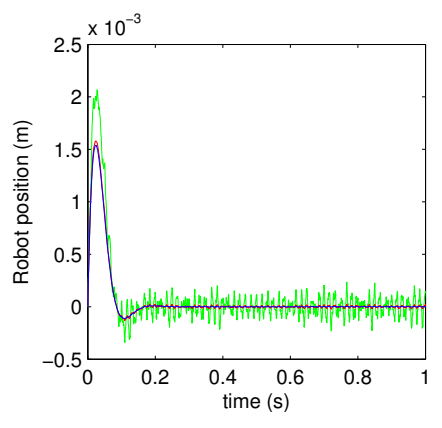

(a) $V_{R}(0)=0.15 \mathrm{~m} \mathrm{~s}^{-1}$

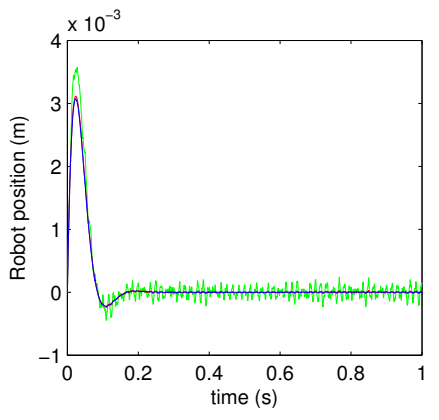

(b) $V_{R}(0)=0.3 \mathrm{~m} \mathrm{~s}^{-1}$

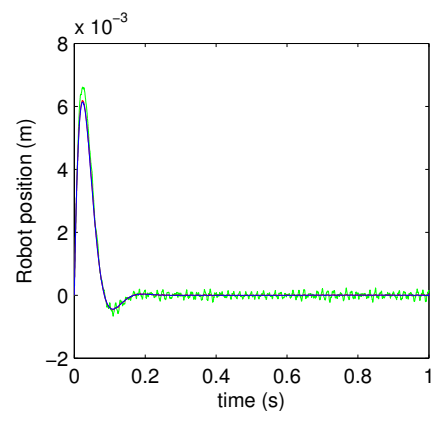

(c) $V_{R}(0)=0.6 \mathrm{~m} \mathrm{~s}^{-1}$

Figure A.17: Robot motion results of the discretely controlled system with levels of sensor noise impacting a rigid environment. The blue, red and green lines indicate the measured noise, $10 \times$ the measured noise and $100 \times$ the measured noise, respectively. 


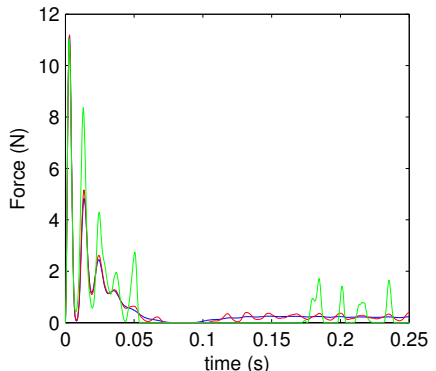

(a) $V_{H}(0)=0.15 \mathrm{~m} \mathrm{~s}^{-1}$

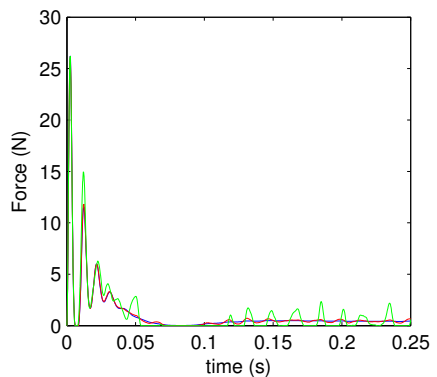

(b) $V_{H}(0)=0.3 \mathrm{~m} \mathrm{~s}^{-1}$

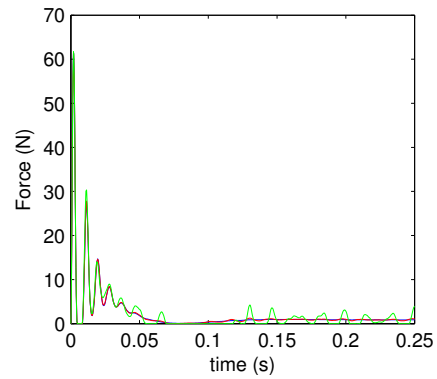

(c) $V_{H}(0)=0.6 \mathrm{~m} \mathrm{~s}^{-1}$

Figure A.18: Contact force results of the discretely controlled system with levels of sensor noise impacting the head model. The blue, red and green lines indicate the measured noise, $10 \times$ the measured noise and $100 \times$ the measured noise, respectively. 


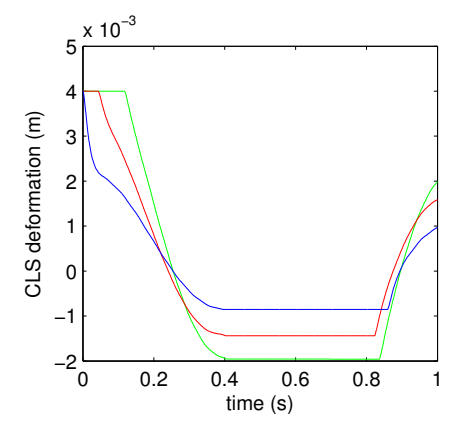

(a) $K_{C}=100 \mathrm{~N} \mathrm{~m}^{-1}$,

$$
B_{C}=\mathbf{0} \text {, }
$$

$M_{E}=0.01 \mathrm{~kg}$,

$K_{E}=100 \mathrm{~N} \mathrm{~m}^{-1}$ \&

$B_{E}=\mathbf{0}$

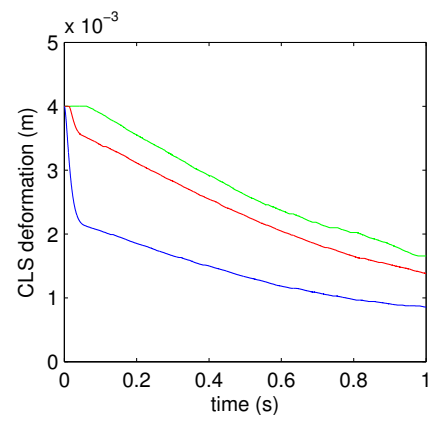

(d) $K_{C}=1000000 \mathrm{~N} \mathrm{~m}^{-1},(\mathrm{e})$

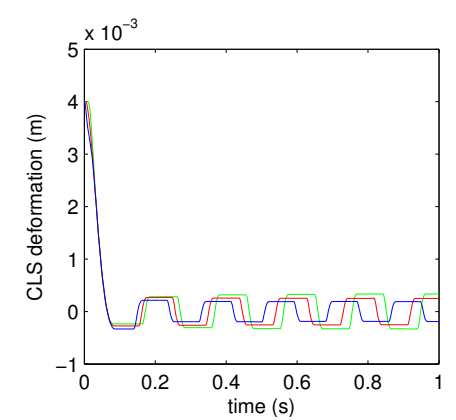

(b) $K_{C}=10000 \mathrm{~N} \mathrm{~m}^{-1}$, $B_{C}=\mathbf{0}$, $M_{E}=0.01 \mathrm{~kg}$, $K_{E}=10000 \mathrm{~N} \mathrm{~m}^{-1}$ \& $B_{E}=\mathbf{0}$

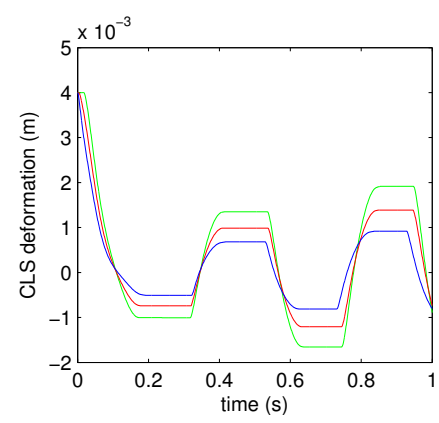

(e) $K_{C}=1000 \mathrm{~N} \mathrm{~m}^{-1}$, $B_{C}=10 \mathrm{~N} \mathrm{~s} \mathrm{~m}^{-1}$, $M_{E}=1 \mathrm{~kg}$, $K_{E}=1000 \mathrm{~N} \mathrm{~m}^{-1} \&$ $B_{E}=10 \mathrm{~N} \mathrm{~s} \mathrm{~m}^{-1}$

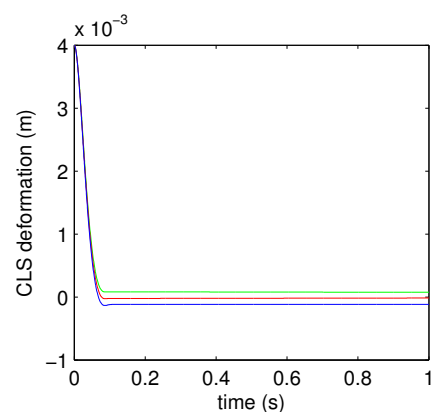

(c) $K_{C}=1000000 \mathrm{~N} \mathrm{~m}^{-1}$, $B_{C}=\mathbf{0}$, $M_{E}=100 \mathrm{~kg}$, $K_{E}=\mathbf{0} \& B_{E}=\mathbf{0}$

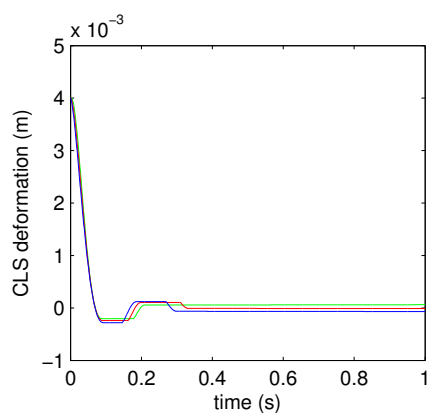

(f) $K_{C}=10000 \mathrm{~N} \mathrm{~m}^{-1}$, $B_{C}=100 \mathrm{~N} \mathrm{~s} \mathrm{~m}^{-1}$, $M_{E}=1 \mathrm{~kg}$, $K_{E}=10000 \mathrm{~N} \mathrm{~m}^{-1}$ \& $B_{E}=100 \mathrm{~N} \mathrm{~s} \mathrm{~m}^{-1}$

Figure A.19: CLS motion results of the controlled system with varying magnitudes of dry friction interacting with the environment. The blue, red and green lines indicate $4 \mathrm{~N}, 6 \mathrm{~N}$ and $8 \mathrm{~N}$ of static friction, respectively. 


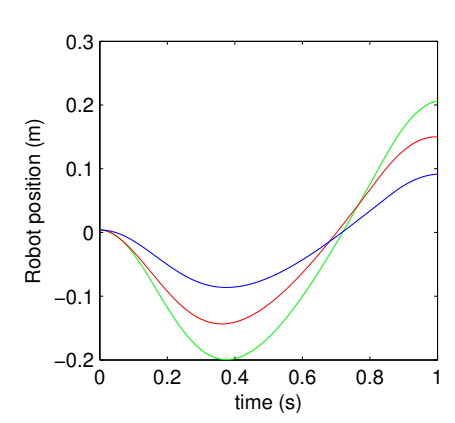

(a) $K_{C}=100 \mathrm{~N} \mathrm{~m}^{-1}$,

$B_{C}=\mathbf{0}$,

$M_{E}=0.01 \mathrm{~kg}$,

$K_{E}=100 \mathrm{~N} \mathrm{~m}^{-1} \&$

$B_{E}=\mathbf{0}$

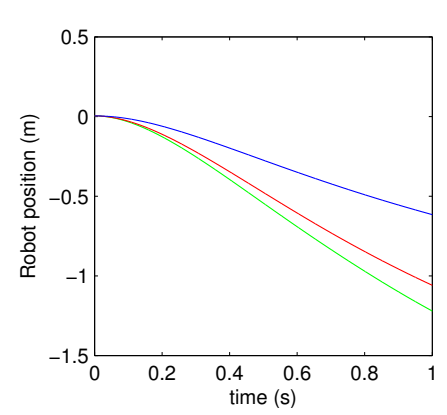

(d) $K_{C}=10000 \mathrm{~N} \mathrm{~m}^{-1}$,

$$
\begin{aligned}
& B_{C}=\mathbf{0}, \\
& M_{E}=0.01 \mathrm{~kg}, \\
& K_{E}=\mathbf{0} \& B_{E}=\mathbf{0}
\end{aligned}
$$
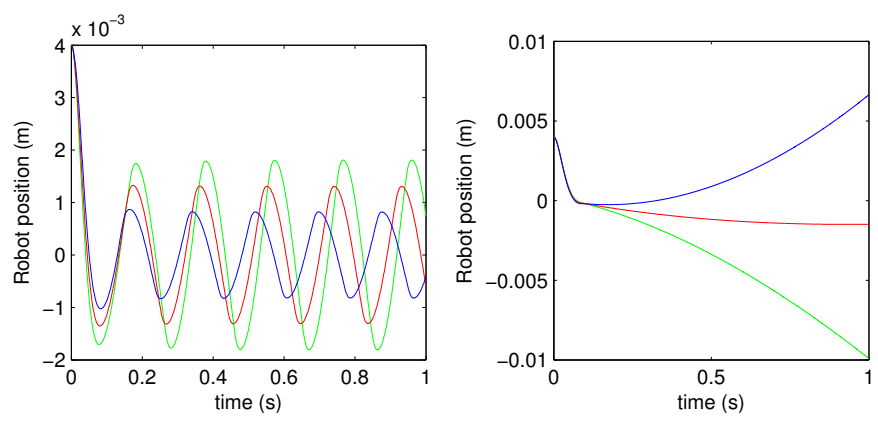

(b) $K_{C}=1000000 \mathrm{~N} \mathrm{~m}^{-1}$, (c)

$$
\begin{aligned}
& B_{C}=\mathbf{0}, \\
& M_{E}=0.01 \mathrm{~kg}, \\
& K_{E}=10000 \mathrm{~N} \mathrm{~m}^{-1} \\
& \& B_{E}=\mathbf{0}
\end{aligned}
$$

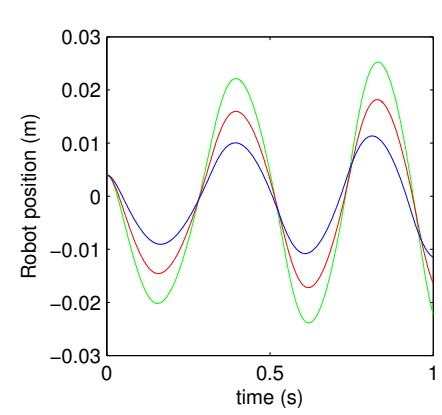
(e) $K_{C}=1000 \mathrm{~N} \mathrm{~m}^{-1}$, $B_{C}=10 \mathrm{~N} \mathrm{~s} \mathrm{~m}^{-1}$, $M_{E}=1 \mathrm{~kg}$, $K_{E}=1000 \mathrm{~N} \mathrm{~m}^{-1} \&$ $B_{E}=10 \mathrm{~N} \mathrm{~s} \mathrm{~m}^{-1}$

Figure A.20: Robot motion results of the controlled system with varying magnitudes of dry friction interacting with the environment. The blue, red and green lines indicate $4 \mathrm{~N}, 6 \mathrm{~N}$ and $8 \mathrm{~N}$ of static friction, respectively. 


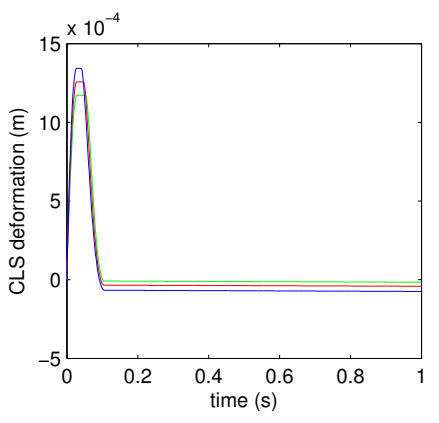

(a) $V_{R}(0)=0.15 \mathrm{~m} \mathrm{~s}^{-1}$

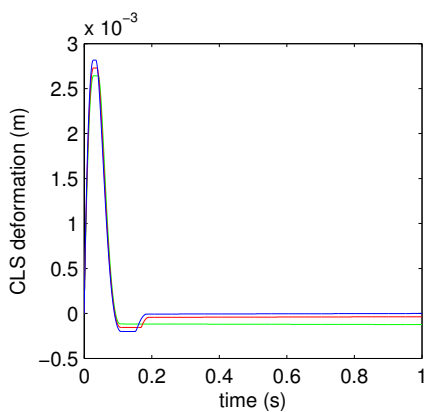

(b) $V_{R}(0)=0.3 \mathrm{~m} \mathrm{~s}^{-1}$

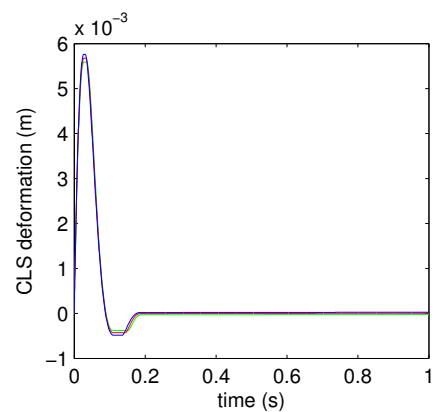

(c) $V_{R}(0)=0.6 \mathrm{~m} \mathrm{~s}^{-1}$

Figure A.21: CLS deflection results of the controlled system with varying magnitudes of dry friction impacting a rigid environment. The blue, red and green lines indicate $4 \mathrm{~N}, 6 \mathrm{~N}$ and $8 \mathrm{~N}$ of static friction, respectively.

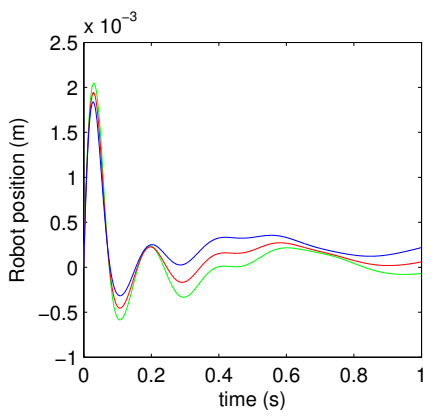

(a) $V_{R}(0)=0.15 \mathrm{~m} \mathrm{~s}^{-1}$

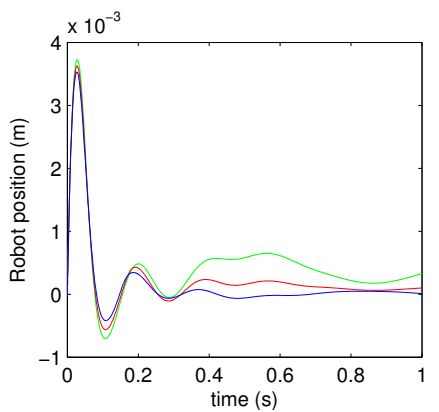

(b) $V_{R}(0)=0.3 \mathrm{~m} \mathrm{~s}^{-1}$

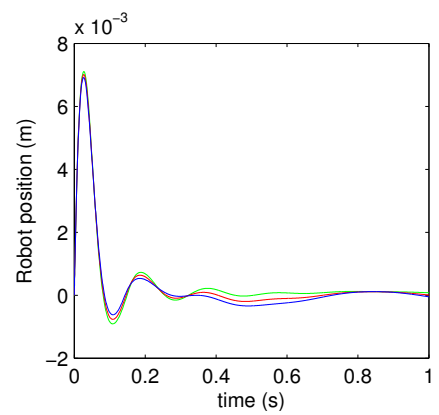

(c) $V_{R}(0)=0.6 \mathrm{~m} \mathrm{~s}^{-1}$

Figure A.22: Robot motion results of the controlled system with varying magnitudes of dry friction impacting a rigid environment. The blue, red and green lines indicate $4 \mathrm{~N}, 6 \mathrm{~N}$ and $8 \mathrm{~N}$ of static friction, respectively. 


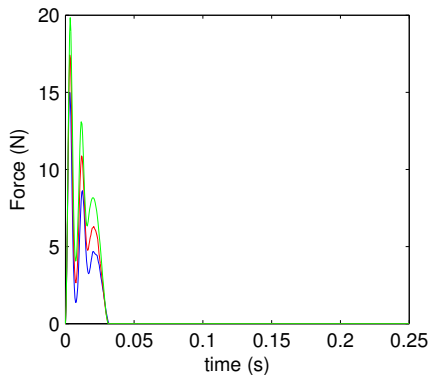

(a) $V_{H}(0)=0.15 \mathrm{~m} \mathrm{~s}^{-1}$

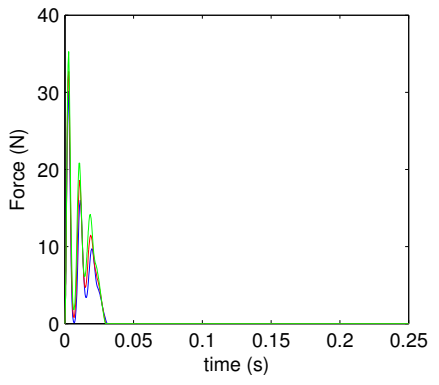

(b) $V_{H}(0)=0.3 \mathrm{~m} \mathrm{~s}^{-1}$

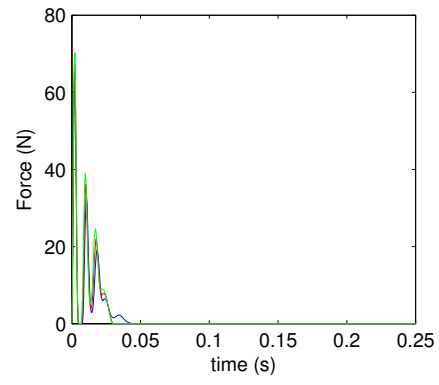

(c) $V_{H}(0)=0.6 \mathrm{~m} \mathrm{~s}^{-1}$

Figure A.23: Contact force results of the controlled system with varying magnitudes of dry friction impacting the head model. The blue, red and green lines indicate $4 \mathrm{~N}, 6 \mathrm{~N}$ and $8 \mathrm{~N}$ of static friction, respectively. 